\title{
Visual Misperceptions and Behavioral Variability in Parkinson's Disease
}

\author{
Dissertation for the award of the degree \\ 'Doctor rerum naturalium' \\ at the Georg-August-Universität Göttingen \\ in the doctoral programme Behavior and Cognition \\ at the Georg-August University School of Science (GAUSS) \\ submitted by
}

Kristina Miloserdov

From

Togliatti, Russian Federation

Göttingen, 2019 


\section{Thesis Committee Members}

Prof. Dr. Melanie Wilke

Institute of Cognitive Neurology

University Medical Center Goettingen

Prof. Dr. Annekathrin Schacht

Dept. of Affective Neuroscience and Psychophysiology

University of Goettingen

Prof. Dr. Hans-Jörg Scherberger

Neurobiology Labratory

German Primate Center

\section{Members of the Examination Board}

Referee: Prof. Dr. Melanie Wilke

Institute of Cognitive Neurology

University Medical Center Goettingen

2nd Referee: Prof. Dr. Annekathrin Schacht

Dept. of Affective Neuroscience and Psychophysiology

University of Goettingen 


\section{Further members of the Examination Board}

Prof. Dr. Hans-Jörg Scherberger

Neurobiology Laboratory

German Primate Center

Prof. Dr. Mathias Bähr

Dept. of Neurology

University Medical Center Goettingen

Dr. Roberto Goya-Maldonado

Dept. of Psychiatry and Psychotherapy

Center for Translational Research in Systems Neuroscience and Psychiatry University Medical Center Goettingen

Prof. Dr. Martin Sommer

Dept. of Clincal Neurophysiology

University Medical Center Goettingen

Oral examination: $9^{\text {th }}$ of July 2019 


\section{Contents}

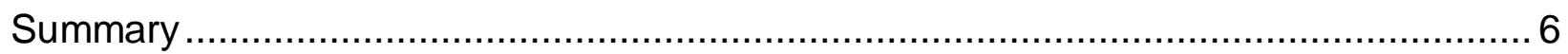

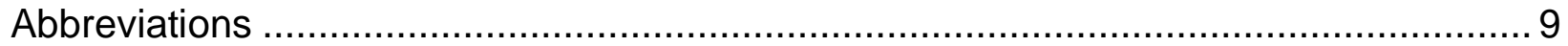

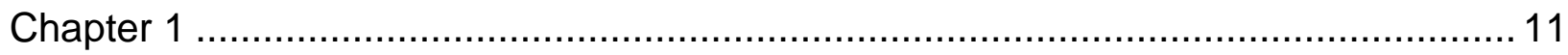

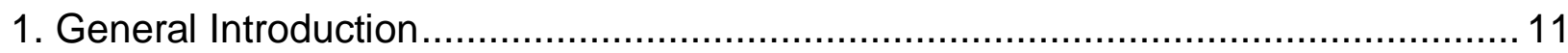

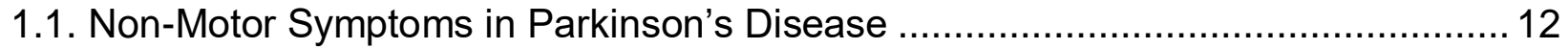

1.2. Neuropsychological Diagnostic and Assesment Tools of Non-Motor Symptoms ... 16

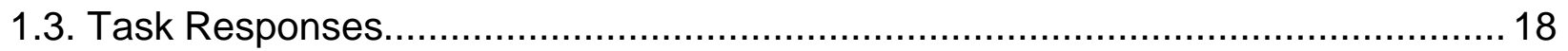

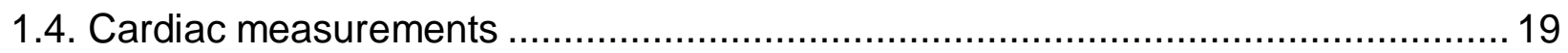

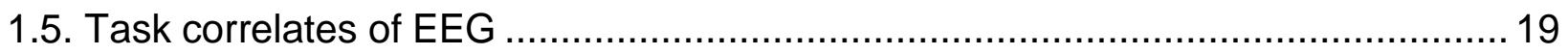

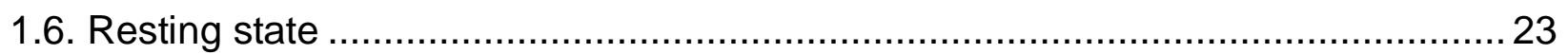

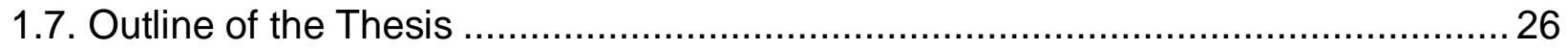

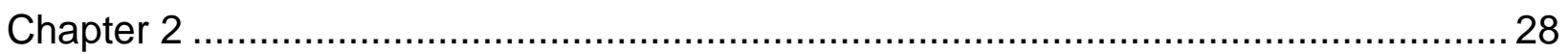

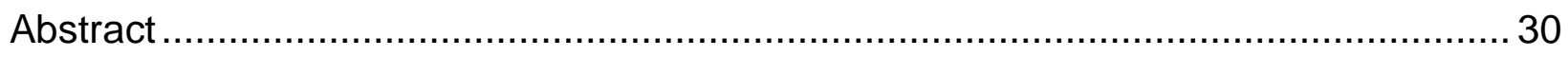

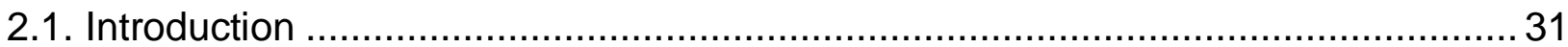

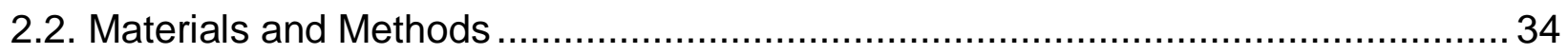

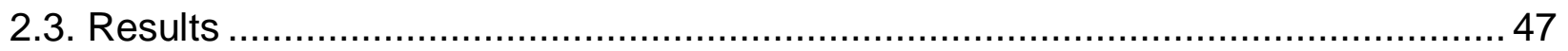

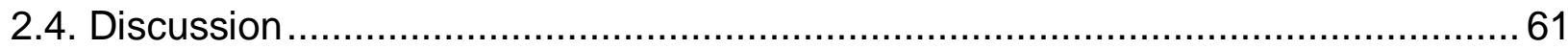

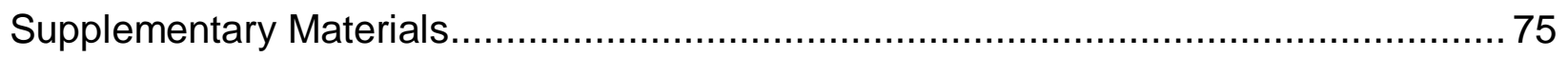

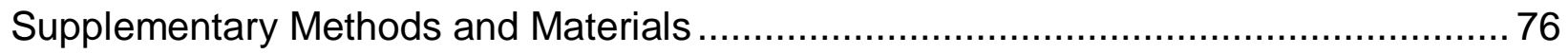

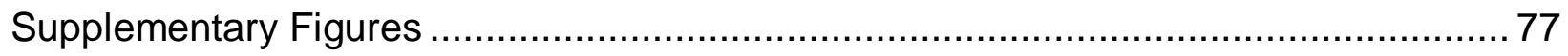

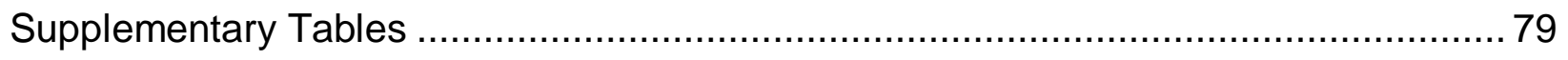

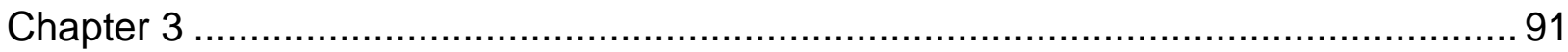

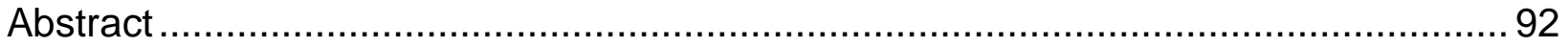

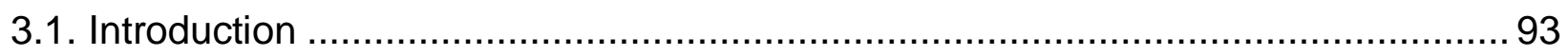

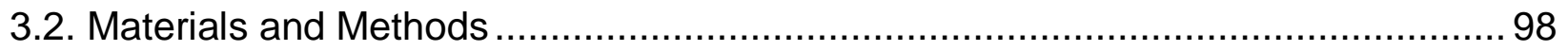

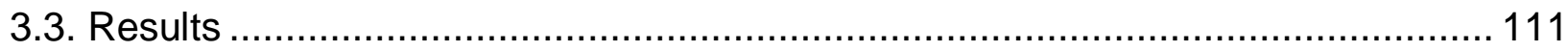

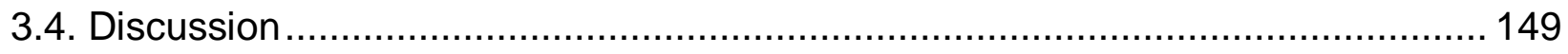

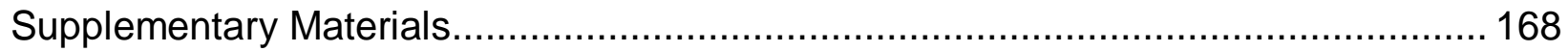

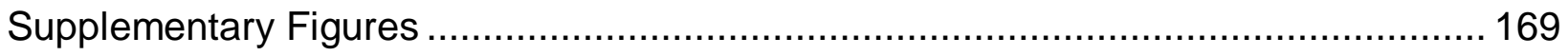

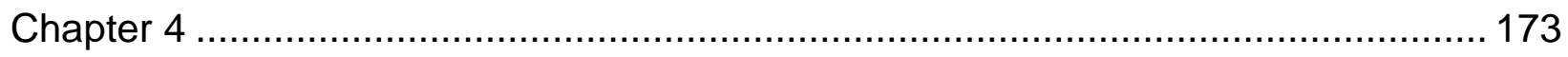

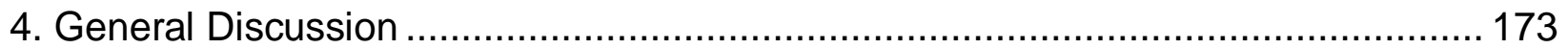


4.1. Non-motor symptoms in Parkinson's disease 173

4.2. Behavioral, neural and cardiac variability in healthy aging ............................... 174

4.3. Behavioral variability in Parkinson's disease .................................................. 176

4.4. Visual Misperceptions and Hallucinations in Parkinson's disease ........................ 177

4.5. Resting state networks in Parkinson's disease.............................................. 178

4.6. Top-down processing in visual misperceptions and behavioral variability in

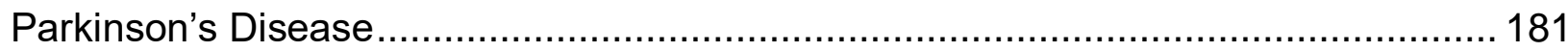

4.7. Diagnostic Tools for differential diagnosis of different PD phenotypes................ 182

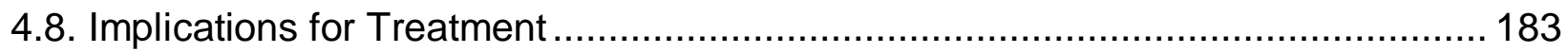

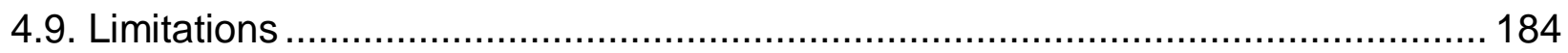

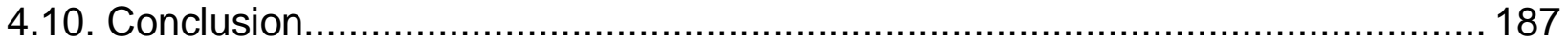

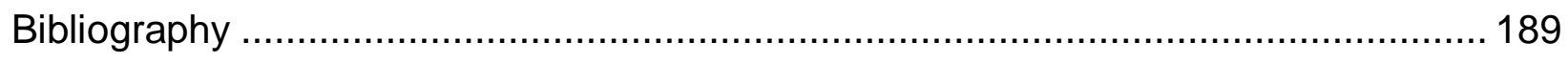

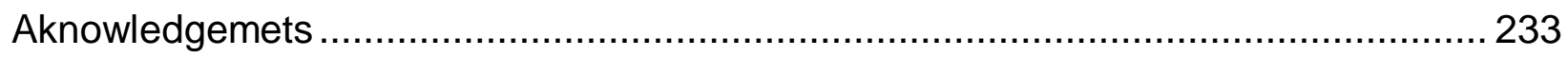

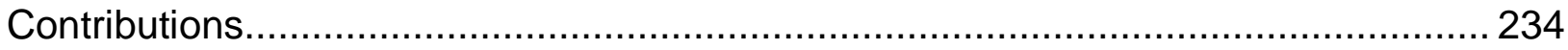




\section{Summary}

The objective of this thesis are visual misperceptions and behavioral variability, their neural correlates and their potential for differential diagnosis of non-motor symptoms profiles in Parkinson's disease (PD). This thesis investigates 1) objective and quantifiable measures of visual misperceptions and behavioral variability in PD and 2) functional connectivity correlates of visual misperceptions and behavioral variability 3) neural and cardiac correlates of behavioral variability in cognitive aging.

In Chapter 2, we investigated task derived objective and quantifiable measures of visual misperceptions and behavioral variability in PD patients reporting visual hallucinations $(\mathrm{VH})$ and PD patients without VH. First, PD patients with and without VH were compared with age matched healthy control group. Second, we compared PD patients reporting $\mathrm{VH}$ with PD patient who did not report $\mathrm{VH}$. In addition, we investigated the relation between severity of perceptual errors, intra-individual variability of recognition times and fuctional connectivity estimates of Resting state networks in PD patients.

We could show that PD patients who reported VH make more percpetual errors and show more intra-individual variability in recogntion time. In a subsample of PD patients, who participated in an functional Magnetic Resonance Imaging (fMRI) resting state experiment, a higher perceptual error score was related to hypoconnectivity between dorsal attention- and salience network. Increased intra-individual variability with respect to recognition time was related to hypoconnectivity between the somatomotor- and right fronto-parietal network.

Perceptual errors and the hypoconnectivity between attentional networks might be a promising objective measurement to estimate the risk for $\mathrm{VH}$ of PD patients. The 
determination of behavioral variability and hypoconnectivity between the somatomotorand fronto-parietal network might be a useful to determine cogntive decline.

Behavioral variability is increased in different disorders like attention deficit hyperactivity disorder (ADHD), obsessive-compulsive disorder (OCD), impulse control disorder (ICD) (Abramovitch, McCormack, Brunner, Johnson, \& Wofford, 2019; Kertzman et al., 2018; Kofler et al., 2013), schizophrenia (Rentrop et al., 2010), dementia (Costa, Dogan, Schulz, \& Reetz, 2019), Huntington Disease (Musso et al., 2015) and in untreated PD patients (Burton, Strauss, Hultsch, Moll, \& Hunter, 2006; Camicioli, Wieler, de Frias, \& Martin, 2008) which are characterized by cognitive decline. Until now the source of behavioral variability is not fully understood. Behavioral variability might be explained by variability quenching, a novel measurement which is until now investigated in healthy young individuals (Arazi, Censor, \& Dinstein, 2017; Arazi, Gonen-Yaacovi, \& Dinstein, 2017). In Chapter 3 we investigated age related cognitive decline. First, we compared behavioral variability, event-related potentials such as $\mathrm{P} 1$ and $\mathrm{P} 3$, change in power of alpha, beta, gamma and theta frequency, neural variability (variability quenching) and cardiac variability (HRV) between healthy elderly and healthy young volunteers. Second we tested the relation between standardized cognitive measures, task derived measures such as intra-individual variability, neural measures and cardiac measures in healthy young and healthy elderly. Healthy elderly show higher behavioral variability, lower P1 and P3 amplitude, lower theta power and lower cardiac variability. Lower cognitive performance in healthy elderly is related to higher behavioral variability and to lower neural variabiltiy. To conclude the measurement of behavioral- and neural variability are promising marker of cognitive decline. Perceptual errors in combination with hypoconnectivity between the dorsal attention network and the salience network 
might be a promising tool to indicate PD patients at risk for experience of visual misperceptions and visual hallucinations. The analysis of behavioral variability combined with hypoconnectivity between somatomotor- and the fronto-parietal network might be a promising tool to identify PD patients at risk for cognitive decline. 


\section{Abbreviations}

ADHD: attention deficit hyperactivity disorder

BDI: Beck's Depression Inventory

CFS: Continuous Flash Suppression

CNS: Central Nervous System

CVRT: Coefficient of Variability in Recognition Time

DAN: dorsal attention network

DMN: default mode network

ECG: Electrocardiogram

EEG: Electroencephalogram

fMRI: functional Magnetic Resonance Imaging

HRV: Heart Rate Variability

LEDD: Levodopa-Equivalent Daily Dose

LB: Lewy Bodies

MCI: Mild Cognitive Impairment

MMSE: Mini Mental State Examination

PD: Parkinson's Disease

PD-MCl: mild cognitively impaired PD

PD-nonVH: Parkinson patients without visual hallucinations

PD-VH: Parkinson patients with visual hallucinations

PES: Perceptual Error Score

RBD: Rapid eye movement disorder

SAL: salience network

TMT: Trail Making Test

OCD: obsessive-compulsive disorder

ICA: Independent Component Analysis

ICD: impulse control disorder

VAN: central attention network 
VH: visual hallucinations

UM-PDHQ: University of Miami Parkinson's disease Hallucinations Questionnaire 


\section{Chapter 1}

\section{General Introduction}

Parkinsons' Disease (PD) is a neurodegenerative disorder which progresses in motor and non-motor symptoms (Nagatsu, Nakashima, Ichinose, \& Kobayashi, 2018). It is variable in disease onset and course, having a diverse clinical picture (Sethi, 2002). To classify the heterogeneous symptoms disease stages as well as subtyping in different syndromes are used (Linazasoro, 2007). Classically, PD is known as a hypokinetic movement disorder with its prominent motor symptoms such as bradykinesia, rigidity and resting tremor. These motor symptoms result from dysfunction of the Central Nervous System (CNS) due to neuronal loss in the Substantia Nigra pars compacta and occurrence of abnormal cell deposits, Lewy Bodies (LB) (Graybiel, 2005; Kalia \& Lang, 2015). In addition to the CNS other brain structures which are part of the autonomous nevous system such as the cerebellum, locus coeruleus and the pedunculopoontine nucleus are affected (Braak \& Del Tredici, 2017; Kalia \& Lang, 2015; Obeso et al., 2017). This indicates that PD is a syndrome where dysfunction of multiple systems is involved, which might provide explanation for non-motor symptoms with involvement of autonoumous dysfunction (Braak \& Del Tredici, 2017; Kalia \& Lang, 2015; Obeso et al., 2017). Dysfunction of the fronto-parietal network seems to be involved in cognitive impairment and visual hallucinations (Haber, 2014; Gratwicke, Jahanshahi \& Foltynie, 2015; Trojano \& Papagno, 2018). The involvement of different neural systems might explain the diversity in symptome profiles including motor and non-motor symptoms in PD. 


\subsection{Non-Motor Symptoms in Parkinson's Disease}

Different neural pathways such as the negrostiatal pathway which is important for movement and the mesolimbic pathway which is important for reinforcement and emotional processes, are affected in PD (Trojano \& Papagno, 2018). Disruption of these pathways might contribute to impairments in different non-motor domains (Trojano \& Papagno, 2018). PD patients show therefore diverse non-motor symptoms such as sleep, sensory, autonomic, cognitive and neuropsychiatric disturbances (Adler, 2011; Park \& Stacy, 2009; Reichmann, Brandt, \& Klingelhoefer, 2016; Trojano \& Papagno, 2018).

\subsubsection{Sensory}

Sensory changes consist of olfactory decrease, pain, restless legs syndrome and visual impairments (Park \& Stacy, 2009). Early symptoms are hyposmia experienced in 70 $100 \%$ of PD patients (Katzenschlager \& Lees, 2004) and shoulder pain (Stamey, Davidson, \& Jankovic, 2008). Chronic pain is experienced by $40 \%$ of PD patients and coocurs with increase in other complains such as motor symptoms and mood changes or higher depression (Nègre-Pagès et al., 2008). Diverse and progressive deficits (Levin et al., 1991) can occur at an early stage (Foltynie, Brayne, Robbins, \& Barker, 2004) in areas of visual perception such as visual acuity, contrast sensitivity, color discrimination, motion perception, peripheral visual field sensitivity and visual processing speed (Diederich, Raman, Leurgans, \& Goetz, 2002; Uc et al., 2005), perception of extrapersonal space (Montse, Pere, Carme, Francesc, \& Eduardo, 2001) and facial recognition (Kida, Tachibana, Takeda, Yoshikawa, \& Okita, 2007). 


\subsubsection{Sleep}

Sleep disorders such as early morning awakenings, sleepiness during the day, sleep attacks, advanced sleep phase syndrome, and rapid eye movement disorder (RBD) occur in 60 to $98 \%$ of PD patients (Stacy, 2002). RBD is characterized by acting out dreams during rapid eye movement sleep phase. Interestingly, RBD is an early, even premotor symptom of PD (Adler, 2011). Moreover, RBD and PD patiens show autonomic dysfunction indicated by reduced heart rate variability (HRV) (Valappil et al., 2010). Furthermore, RBD is related to more rapid progression of PD and occurrence of dementia and hallucinations (Fereshtehnejad et al., 2015; Kim \& Jeon, 2014; Romenets et al., 2012).

\subsubsection{Autonomic dysfunction}

Autonomic disturbances consist of nausea, constipation, excessive sweating, constipation, urogenital problems, cardiac problems, orthostatic hypotension and HRV (Adler, 2011; Park \& Stacy, 2009). Constipation is frequently reported and precedes motor symptoms (Abbott et al., 2001). An early occuring cardiac abnormality is the myocardial postganglionic sympathetic dysfunction (Iwasa et al., 1998). Decrease in HRV occurs also in PD patients who were not exposed to medication and might be a potential marker at an early disease stage (Ferini-Strambi, Franceschi, Pinto, Zucconi, \& Smirne, 1992; Kallio et al., 2004; Mihci, Kardelen, Dora, \& Balkan, 2006).

\subsubsection{Neuropsychiatric disturbances}

Neuropsychiatric disturbances include apathy, depression, anxiety, disorders of emotional processing, impulse control disorders, cognitive impairment, psychosis and 
hallucinations (Bertram \& Williams, 2012; Cosgrove, Alty, \& Jamieson, 2015; Eversfield \& Orton, 2018; Trojano \& Papagno, 2018). Depression and anxiety are associated in PD and occur in 19\%, respectively $30 \%$ of PD patients (Broen, Narayen, Kuijf, Dissanayaka, \& Leentjens, 2016; Goodarzi et al., 2016). Both are related to other nonmotor symptoms. Anxiety predicts cognitive decline and depression which is related to executive dysfunction and apathy (Pirogovsky-Turk et al., 2017; Santangelo et al., 2015; Varanese et al., 2010). Apathy occurs in $20 \%$ of PD patients and is present in the prodromal phase (Pagonabarraga, Kulisevsky, Strafella, \& Krack, 2015; Varanese, Perfetti, Ghilardi, \& Di Rocco, 2011). Apathy is also associated with executive impairments and predicts development of dementia (Santangelo et al., 2015). Another non-motor symptom, which is related to executive impairments is impulse control disorders (ICD) consisting of compulsive behavioral manifestations such as gambling, compulsive shopping and hypersexuality (Santangelo et al., 2007; Weintraub et al., 2015). It is already present at an early disease stage but increases with dopaminergic medication (de la Riva, Smith, Xie, \& Weintraub, 2014). Most of the neuropsychiatric symptoms, apathy, depression, anxiety and ICB are related to cognitive changes indicating a general cognitive dysfunction in PD. Cognitive changes such as dementia are diagnosed with dementia in approximately $30 \%$ of the PD patients (Park \& Stacy). These demented patients are characterized with deficits in information processing speed, especially in visuospatial, attentional and executive processing and fluctuations in attention (Lees \& Smith, 1983; Park \& Stacy, 2009). 


\subsubsection{Visual Hallucinations}

Visual hallucinations $(\mathrm{VH})$ are perceptions without the presense of an external stimulus. Visual misperceptions are illusory or distorted percepts in presence of an external stimulus (Bertram \& Williams, 2012). VH prevalence in PD is between $15-75 \%$ (Aarsland et al., 1999; Eversfield \& Orton, 2018; Fénelon, Mahieux, Huon, \& Ziégler, 2000; Hely, Reid, Adena, Halliday, \& Morris, 2008; Sanchez-Ramos, Ortoll, \& Paulson, 1996; Williams \& Lees, 2005). Variance in prevalence can be explained by different study methods (Bertram \& Williams, 2012). Studies cooperating with specialized movement disorders facilities report a prevalence between 25 to $50 \%$ (Fénelon et al., 2000; Sanchez-Ramos et al., 1996). Most studies described occurrence of complex or formed VH such as people, animals or objects (Bertram \& Williams, 2012). Minor hallucinations consisting of visual misperceptions, passage hallucinations (sense of movement in the periphery) and 'presence' hallucinations (feeling of a presence in the room) might be prehallucinatory symptoms (Fénelon et al., 2000) proceeding to psychosis with occurrence of delusion and loss of insight into hallucinations (Goetz \& Stebbins, 1993). PD patients do mostly not report minor hallucinations or misperceptions as hallucinatory experiences. These experiences are only shared by direct questioning (Williams, Warren, \& Lees, 2008). This fact brings to light that measuring visual misperceptions might be usefull for recognizing individuals at risk for development of $\mathrm{VH}$. In general, $\mathrm{VH}$ are reported in the later stages of PD (Williams \& Lees, 2005). However, we do not know whether the occurrence of VH is simply underreported at earlier stages because patients are not feeling comfortable to report the experience of $\mathrm{VH}$ due to the fear of being judged or because they may not be aware of the hallucinatory/illusory experience. Given that $\mathrm{VH}$ is a reliable predictor of dementia 
(Lee, Tsai, Gauthier, Wang, \& Fuh, 2012; Santangelo et al., 2007) with 70\% prevalence (Fénelon et al., 2000) recognizing risk groups for development of $\mathrm{VH}$ and cognitive decline might improve management of the symptoms and thereby reduce distress in patients and their caregivers (Dudley et al., 2012). Patients might be unaware of, underestimate, underreport or hide the experience of socially undesirable symptoms such as VH. For this reason, it is important to have an objective and quantifiable assessment tools for $\mathrm{VH}$. These tools can provide accurate differential diagnosis to correctly inform the patient about their diagnosis and its prognosis, to plan adequate treatment and possibly evaluate treatment in the future.

\subsection{Neuropsychological Diagnostic and Assesment Tools of Non-Motor}

\section{Symptoms}

Non-motor symptoms are measured by patient report with standardized non-motor symptoms questionnaires such as the Non-motor Symptom Questionnaire (NMSQuest) (Chaudhuri et al., 2006). It is assessing gastrointestinal symptoms, urinary symptoms, sexual function, sleep/fatique, pain and miscellaneous symptoms, and Non-Motor Symptoms Scale (NMSS) (Chaudhuri et al., 2007) containing items about cardiovascular symptoms, sleep/fatique, mood, cognition, perception, sexual function and miscellany symptoms (Goldman et al., 2014). As reviewed in (Kulisevsky \& Pagonabarraga, 2009) different rating scales asses different symptoms such as sleep with the Epwoth Sleepiness Scale (ESS), Pittsburg Sleep Quality Index (PSQI), fatique with the Fatique Severity Scale and autonomic symptoms with the SCOPA-autonomic scale (Goldman \& Postuma, 2014). Impulse control disorders such as compulsive gambling, shopping, sexual behavior and eating are measured with the Questionnaire 
for Impulsive-Compulsive Disorders in Parkinson's Disease (Weintraub, Papay, Siderowf, \& Parkinson's Progression Markers Initiative, 2013). Often cognitive symptoms are measured using scales which are not specific for PD (Kulisevsky \& Pagonabarraga, 2009) such as the Montreal Cognitive Assessment (MoCA), MiniMental State Examination (MMSE) (Goldman \& Postuma, 2014), Cambridge Cognitive Assessment, Frontal Assessment Battery (FAB) and Mattis Dementia Rating Scale (Kulisevsky \& Pagonabarraga, 2009). To detect early cognitive deficits in PD the Scales for Outcomes of Parkinson's Diasese Cognition and the Parkinson's Disease Cognitive Rating Scale, which is also sensitive to the transition to dementia in PD, are recommended (Kulisevsky \& Pagonabarraga, 2009). Mattis Dementia Rating Scale shows excellent discrimination for dementia in PD (Kulisevsky \& Pagonabarraga, 2009). Also given that $\mathrm{VH}$ often occur in dementia additional screening tools are needed to accurately assess this symptom. Patients do not always want to share their hallucinatory experience. Clinicians and researchers need to interview the patients to classify these symptoms. Rating scales can be used to standardize the interview procedure and to scale the severity of the hallucinatory experience. Rating scales are available but there is no standardized procedure for assessment of halucinations in PD (Kulisevsky \& Pagonabarraga, 2009). Hallucinations can be measured with the Neuropsychiatiric Inventory, item 2 of the Movement Disorders Society Unified Parksinons's Disease Rating Scale (MDS-UPDRS) (Kulisevsky \& Pagonabarraga, 2009) and the Parkinson Psychosis Rating Scale (Fernandez et al., 2008). Separate assessment of $\mathrm{VH}$ is possible with the University of Miami Parkinson's disease Hallucinations Questionnaire UM-PDHQ (Papapetropoulos et al., 2008). However, 
this assesment neglects the full range of hallucinatory experience such as minor hallucinations, visual misperceptions and passage hallucinations.

The above described procedures provide standardization to the assessment of nonmotor symptoms. However, these measurements has still the weaknes not assesing the full range of symptoms such as $\mathrm{VH}$ and they are not objective because the patient can still deceive him- or herself and the examinator. To prevent this, objective measurements can be derived from responses to a task. These can be used to detect patient groups at risk for different symptom profiles.

\subsection{Task Responses}

In an object recognition task with noise degraded images, hallucinating PD patients needed more time for image recognition (Meppelink, Koerts, Borg, Leenders, \& van Laar, 2008). In reaction time tasks performance variability predicted transition from $\mathrm{MCl}$ to dementia (Gorus, De Raedt, Lambert, Lemper, \& Mets, 2008; Tales et al., 2012). Interestingly, increase in behavioral variability charcterized PD patients with $\mathrm{MCl}$ and dementia (de Frias, Dixon, \& Camicioli, 2012). Previous research in hallucinating PD patients showed that these patients make more perceptual errors in a task using ambiguous images to PD patients who did not report experience of VH (Shine, Keogh, et al., 2015). Thus, different symptom profiles in PD are characterized by differences in task derived responses such as hallucinating PD patient by increased in perceptual errors and cognitively impaired PD patients by increased behavioral variability.

The above described approach using task dereived responses impoves objectivity of the measure identifying patients being at risk for $\mathrm{VH}$. However, patients still could try to 
show desirable behavior by responding in a way they think they are expected to perform or simply misunderstanding, confuse or forget the instructions. To improve this, the task can be combined with cardiac and neural measurements such as electrocardiogram (ECG) and electroencephalogram (EEG) respectively.

\subsection{Cardiac measurements}

The cardiac function is influenced by parasympathetic supression via cingulate cortex and sympathetic activation via the insula. These are part of the Ventral Attention Network involved in attentional dysfunction of the hallucinating PD patients (Shine et al., 2015). As already mentioned previously, lower HRV is related to higher risk of PD and might be an early marker of PD (Ferini-Strambi et al., 1992). Whether HRV might also indicate other non-motor changes such as executive dysfunctions is unknown. In other groups such as healthy elderly HRV seems to be an indicator of self-regulatory control with higher HRV during Resting state being related to better performance in executive functions (Shaffer, McCraty, \& Zerr, 2014) and lower HRV to cognitive decline (Frewen et al., 2013). Cognitive changes in healthy elderly might be objectively measured by behavioral variability and reflected in cardiac variability. However, both measurements might be modulated by neural processes such as neural variability and objectively indicated by EEG derived measures.

\subsection{Task correlates of EEG}

Different task related neural measurements can be derrived from EEG combined with a task. Visually evoked Potentials allow investigation of time-dependent aspects of distinct visual processes, reflecting dysfunction in form of amplitude changes (Matsui et al., 
2006), changes in spectral power of different frequencies and neural variability (Arazi, Censor, et al., 2017; Arazi, Gonen-Yaacovi, et al., 2017).

\subsubsection{Neural variability and cognitive changes in healthy populations}

Non-motor symptoms such as cognitive changes might be indicated by performance fluctuations at the behavioral level. However, neural variability might provide a more objective tool to measure cognitive changes. One possible measurement of neural variability is variability quenching, described as the reduction of neural variability after stimulus presentation. Higher variability quenching correlates with better perceptual performance in healthy young individuals (Arazi, Censor, et al., 2017). Older and slower performing adults showed less brain variability (Garrett, Kovacevic, Mclntosh, \& Grady, 2013), indicating its potential as objective measures of cognitive changes. Neural variability magnitudes measured by EEG recordings are stable over time and across tasks in healthy young human volunteers (Arazi, Gonen-Yaacovi, et al., 2017) showing its reliability as a possible marker of cognitive changes. Before testing neural variability in clinical populations its sensitivity in relation to behavioral variability, standardized cognitive measures and cardiac variability needs to be validated in populations showing age related cognitive changes to prove its potential indicating subtle cognitive changes. Neural variability is measured by variability quenching as a marker of age related cognitive changes was not yet investigated in different healthy populations such as healthy young and healthy elderly. Age related cognitive changes in relation to behavioral, neural and cardiac variability might provide interesting insights into variability correlates of cognitive aging. 


\subsubsection{Parkinson's disease}

Non-motor symptoms in age related neurodegenerative disorders such as PD are related to changes in neural measurements such as EEG (Geraedts et al., 2018). Previous EEG research had shown that different frequency bands are related to different functions in PD. Depression in PD is expressed in lower alpha (Filipović, Covicković-Sternić, Stojanović-Svetel, Lecić, \& Kostić, 1998). Decrease in alpha and beta power reflects cognitive dysfunction (Caviness et al., 2007; Soikkeli, Partanen, Soininen, Pääkkönen, \& Riekkinen, 1991) and increase in beta power (He, Zhang, Chen, Xie, Gan, Yang, et al., 2017) and alpha amplitude is related to motor symptoms such as the Hoehn and Yahr stage of PD (Fonseca, Tedrus, Letro, \& Bossoni, 2009). Higher beta power is related to longer disease duration (Moisello et al., 2015). L-dopa administration can increase alpha and beta power (Melgari et al., 2014). These findings indicate that alpha and beta reflect motor and non-motor changes such as mood and cognitive dysfunction in PD.

Higher theta power correlates with motor symptoms such as higher desease stage $(\mathrm{He}$, Zhang, Chen, Xie, Gan, Wang, et al., 2017) and non-motor symptoms such as higer REM sleep disorder scores (Gagnon et al., 2004) and cognitive decline (Bonanni et al., 2008; Caviness et al., 2007, 2016; Fonseca et al., 2009; Pozzi et al., 1994; Soikkeli et al., 1991). Moreover, higher theta power is predictive for cognitive deterioration in PD indicating its potential as a marker of cognitve decline (Caviness et al., 2016; Cozac et al., 2016; Klassen et al., 2011). 


\subsubsection{Visual Halucinations}

Previous research in different groups susceptible to hallucinatory experience proposes different interesting possible marker of $\mathrm{VH}$ derived from EEG. Previous research had shown that healthy individuals susceptible to $\mathrm{VH}$ have increased $\mathrm{P} 1$ amplitude (Schwartzman, Maravic, Kranczioch, \& Barnes, 2008) and increased gamma synchronization (Becker, Gramann, Müller, \& Elliott, 2009). Studies in clinical groups experience VH such as PD patients had shown deviant P1 (Matsui et al., 2006), delayed P200 (Kurita, Murakami, Takagi, Matsushima, \& Suzuki, 2010), increased P3 latencies (daSilva Morgan, Elder, Ffytche, Collerton, \& Taylor, 2018; Kurita et al., 2010) and decreased alpha power (Bonanni et al., 2008; Bosboom, Stoffers, Stam, Berendse, \& Wolters, 2009). Alzheimer patients showed increased occipital and temporal theta activity (Lopez et al., 1991) and in LB dementia reduced theta synchronization (Peraza et al., 2018). Changes in $\mathrm{P} 1, \mathrm{P} 3$, alpha, gamma and theta power might be potential markers of VH in PD. Given that P1 is an early perceptual component it should reflect perceptual deficits and P3 as a later more cognitive influenced component reflecting attentional and cognitive deficits (daSilva Morgan et al., 2018).

The above described method using task evoked EEG measures are objective, quantifable and not biased by desirable behavior or misunderstanding of task instructions. However, these measurements might still be influenced by the unintentional suggestions of the experimenter that one kind of stimuli is more important than another resulting in more attention of the subject to a specific stimulus condition. Another difficulty is the compliance or availability of ressources such as attention by the volunteer. Especially more cognitively or executively impaired volunteers such as PD 
patients suffering from stronger disease progression have difficulties to keep the mandatory level of attention, understand and remember the instructions. To improve this limitations, neural and cardiac activity can be measured while the volunteer is resting.

\subsection{Resting state}

Resting state describes data collection during a task-free, so called resting period. Volunteers are instructed to lay still and stay awake while keeping eyes open or closed for around 6 to 10 minutes, without executing a specfic task and let their mind wander without focusing on a specific topic. In the eyes open resting state a fixation object such as a fixtion cross in the center of the monitor is presented to prevent eye-movements (Vanderwal, Kelly, Eilbott, Mayes, \& Castellanos, 2015). There is more than one possiblity to analyze resting state fMRI data. In general, analyses can be categorized in two approaches, 1) model-dependent such as correlating the data of a predefined brain regions ("seed") against the time-series of other regions, 2) model-free methods such as principal conmponent analysis or independent component analysis (ICA) where connectivity patterns are analyzed without predefining "seed" regions (van den Heuvel \& Hulshoff Pol, 2010). Figure 1.1 shows an overview of the most common Resting state networks, such as the default mode network (DMN), sensorimotor network, insulartemporal network, salience network, parieto-frontal network and the visual network (Prell, 2018). Relating functional connectivity estimates of within and between network connectivity and symptom severity of different symptom profiles might provide potential diagnostic candidates for risk groups with certain symptoms profiles (Hohenfeld, Werner, \& Reetz, 2018). 


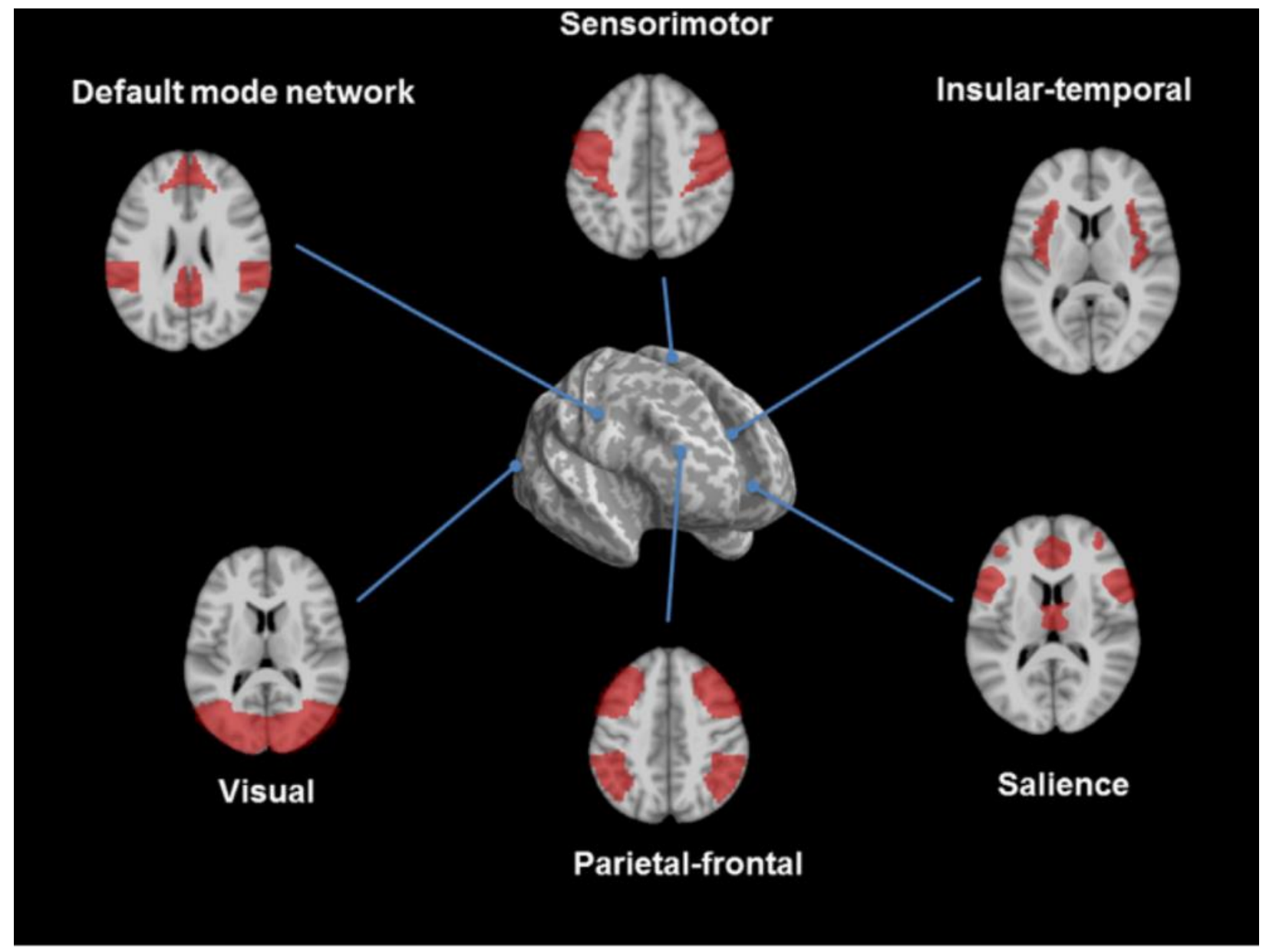

Figure 1.1: Overview of resting state networks. The figure summarizes resting-state networks, functionally related regions, such as the default mode network, sensorimotor, insular-temporal and anterior cingulate cortex regions, salience, executive control and the visual network (Prell, 2018).

\subsubsection{Imaging of non-motor symptoms}

\subsubsection{Fatique}

Tiredness or fatique in PD is related to activity of frontal cortex and anterior cingulate (Li et al., 2017) and hypoconnectivity of temoral, parietal and motor cortices (Zhang et al., 2017). Fatique is related to hypoconnectivity of sensorimotor and DMN in PD (Tessitore et al., 2016). Fatique seems to be characterized by reduced FC between sensorimotor and DMN and within the Ventral Attention Network (VAN). 


\subsubsection{Impulse Control Behavior}

In general impulsive control behaviors (ICB) such as gambling, binge eating, compulsive sexual behavior and compulsive shopping are related to changes in brain function of fronto-striatal and fronto-limbic circuits (Prell, 2018). Already drug-naïve PD patients show hypoconnectivity between DAN and executive network and hyperconnectivity within the saliency network indicating that cognitive and limbic connectivity changes predict ICB without ivolvement of dopaminergic medication (Tessitore et al., 2017). Moreover, hypersexual PD patients exposed to sexual cues show changes in limbic, paralimbic, temporal, occipital, somatosensory and prefrontal cortices (Politis et al., 2013). In the above described networks different brain areas are involved in multiple dysfunctions of multiple non-motor symptoms such as 1) frontoparietal areas are involved in cognition, fatique and ICB 2) somatosensory/ sensorimotor areas in fatique and ICB indicating probably an common mechanisms to this non-motor symptoms (Prell, 2018).

\subsubsection{Cognition}

Metabolism in prefontal, temporal and parietal cortex is reduced in mild cognitively impaired PD (PD-MCl) and seems to spread to the anterior cingulate cortex (Huang et al., 2008; Yong, Yoon, An, \& Lee, 2007). Hypoconnectivity within the DMN seems also to play a role in cognitive changes in PD (Tahmasian et al., 2017). Connectivity between the dorsal attention networks and insula, as well as frontal areas is reduced in PD-MCI (Baggio et al., 2015). In addition, connectivity between DMN and posterior brain regions is increased in PD-MCl (Baggio et al., 2015). Cognitive changes in PD seem to be related to connectivity of the $\mathrm{DMN}$, dorsal attentional network and frontal brain areas. 


\subsubsection{Visual Halucinations}

Previous Resting state studies show hyperconnectivity within the DMN which is a tasknegative network, active while no task is executed, especially of the fronto-parietal regions in hallucinating PD patients (Franciotti et al., 2013; Yao et al., 2014). Reduced visuospatial memory performance is related to increased functional connectivity between hippocampus and occipito-temporal brain areas in hallucinating PD patients (Yao et al., 2016). However in another sample of hallucinating patients with LB dementia hallucinations were related to functional connectivity of the left fronto-parietal and sensorimotor networks (Peraza et al., 2014). Interestingly, objectively measured perceptual errors in hallucinating PD patients are related to less functional connectivity between the VAN and the dorsal attention network (DAN) providing evidence that $\mathrm{VH}$ might result from attentional impairments (Shine, Keogh, et al., 2015). Resting state functional connectivity of the DMN, visual, sonsorimotor, fronto-parietal and attentional networks seem to be involved in VH in PD.

\subsection{Outline of the Thesis}

The objective of the thesis are visual misperceptions and behavioral variability in PD. This thesis investigates 1) objective and quantifiable measures of visual misperpections and behavioral variability in PD, 2) functional connectivity correlates of visual misperceptions and behavioral variability in PD and 3) neural and cardiac correlates of behavioral variability in cognitive aging comparing healthy young and healthy elderly adults to investigate the potential of different sources of variability as potential marker of age related cognitive decline. In the first part of the thesis, Chapter 2, we investigated task derived objective and quantifiable measures of visual misperceptions and 
behavioral variability in PD patients with $\mathrm{VH}$ and $\mathrm{PD}$ patients without $\mathrm{VH}$ and age matched healthy controls. In addition, we investigated the relation between severity of perceptual errors, intra-individual variability of recognition times and the relation to fuctional connectivity estimates of resting state networks in PD patients. The second part of the thesis investigated cognitive aging and its behavioral, neural and cardiac correlates. Chapter 3, shows differences in behavioral variability, Event-Related Potentials, $\mathrm{P} 1$ and $\mathrm{P} 3$, change in power of alpha, beta, gamma and theta frequency bands, neural variability (variability quenching) and cardiac variability (HRV) between healthy elderly and healthy young voluteers and the relation between standardized cognitive measures, task derived measures such as intra-individual variability, neural measures and cardiac measures. The results of our clinical and healthy samples of visual misperceptions and behavioral variability are discussed in the broader context of differential diagnosis as possible diagnostic marker of visual halluciantions and cognitive decline and its possible implications for phenotype specific treatment. 


\section{Chapter 2}

\section{Misperceptions and intra-individual variability in Parkinson's disease:}

\section{a behavioral and fMRI study}

Kristina Miloserdov ${ }^{1,3,7}$, Carsten Schmidt-Samoa ${ }^{1}$, Kathleen Williams ${ }^{1}$, Christiane Anne Weinrich $^{2}$, Katrin Bürk ${ }^{4}$, Claudia Trenkwalder ${ }^{5,6}$, Mathias Bähr ${ }^{2,4}$, Melanie Wilke $e^{1,3,7^{*}}$

${ }^{1}$ Department of Cognitive Neurology, University Medical Center Goettingen, RobertKoch-Str. 40, Goettingen, 37075, Germany

${ }^{2}$ Department of Neurology, University Medical Center Goettingen, Robert-Koch-Str. 40, Goettingen, 37075, Germany

${ }^{3}$ German Primate Center, Leibniz Institute for Primate Research, Kellnerweg 4, Goettingen, 37077, Germany

${ }^{4}$ Kliniken Schmieder Stuttgart-Gerlingen, Solitudestraße 20, Gerlingen, 70839, Germany

${ }^{5}$ Paracelsus-Elena Klinik, Klinikstraße 16, Kassel, 34128, Germany

${ }^{6}$ Department of Neurosurgery, University Medical Center Goettingen, Robert-Koch-Str. 40, Goettingen, 37075, Germany

${ }^{7}$ Leibniz-Science Campus Primate Cognition, German Primate Center, Kellnerweg 4, Goettingen, 37077, Germany

* Corresponding author: Melanie Wilke, PhD, Department of Cognitive Neurology, University Medical Center Goettingen, Robert-Koch-Str. 40, Goettingen, 37075, Germany 
melanie.wilke@med.uni-goettingen.de

\section{Keywords:}

Parkinson's disease, visual hallucinations, misperceptions, trial-by-trial variability, flash suppression 


\begin{abstract}
Patients with Parkinson's disease (PD) frequently suffer from visual misperceptions and hallucinations, which are difficult to objectify and quantify. We aimed to develop an image recognition task to objectify misperceptions and to assess performance fluctuations in PD patients with and without self-reported hallucinations. Thirty-two nondemented patients with Parkinson's disease (16 with and 16 without self-reported visual hallucinations) and 25 age-matched healthy controls $(\mathrm{HC})$ were tested. Participants performed a dynamic image recognition task with real and scrambled images. We assessed misperception scores and intra-individual variability in recognition times. In a subsample of Parkinson's disease patients $(N=16)$ we related resting state network connectivity to the behavioral outcomes. We found that PD patients with self-reported hallucinations (PD-VH) exhibited higher perceptual error rates and higher intraindividual variability in recognition times than PD patients without visual hallucinations (PD-nonVH). Both, misperceptions and intra-individual variability were negatively correlated with resting state functional connectivity involving frontal and parietal brain regions, albeit in partly different subregions. Consistent with previous research suggesting that hallucinations arise from dysfunction in attentional networks, misperception scores correlated with reduced functional connectivity between the dorsal attention and salience network. Intra-individual variability correlated with decreased connectivity between somatomotor and right fronto-parietal networks. We conclude that our task can detect visual misperceptions that are more prevalent in PD-VH patients. In addition, fluctuating visual performance appear to be a signature of PD-VH patients,
\end{abstract}


which might assist further studies of the underlying pathophysiological mechanisms and cognitive processes.

\subsection{Introduction}

Patients with Parkinson's disease (PD) frequently experience non-motor symptoms such as cognitive and perceptual deficits (Armstrong, 2011). Visual misperceptions and visual hallucinations $(\mathrm{VH})$ involving complex images are highly prevalent, have a negative impact on quality of life and represent a key predictor for dementia with disease progression (Diederich, Fénelon, Stebbins, \& Goetz, 2009). In clinical studies, the presence of hallucinations is typically assessed with questionnaires such as the University of Miami Parkinson's disease Hallucinations Questionnaire (UMPDHQ) (Papapetropoulos et al., 2008) that do not discriminate between hallucinations and misperceptions and thus these concepts appear entangled in the majority of studies (for exceptions see: (Shine, Halliday, Carlos, Naismith, \& Lewis, 2012)). Visual hallucinations are more likely to occur at advanced disease stages and are co-morbid with REM sleep disorder (Manni et al., 2011), cognitive and attentional dysfunction (Koerts et al., 2010; Meppelink et al., 2008; Shine, Halliday, et al., 2014) as well as with sensory impairments such as reduced visual acuity, color and contrast sensitivity (Matsui et al., 2006; Pieri, Diederich, Raman, \& Goetz, 2000; Ramirez-Ruiz, Junque, Marti, Valldeoriola, \& Tolosa, 2007). These influencing factors suggest that hallucinations in PD patients are modulated by both top-down and bottom-up, sensory deficits (Muller, Shine, Halliday, \& Lewis, 2014). Functional models further implicate that VH's occur as a consequence of reality monitoring due to the misattribution of self- 
generated information (Collerton, Perry, \& McKeith, 2005). A recent hypothesis of dysfunctional connectivity between the dorsal attention network (DAN) and the ventral attention network (VAN) arises from functional imaging studies in PD patients (Shine, Keogh, et al., 2015). Specifically, Shine, Keogh, et al., 2015 showed that impaired functional connectivity is related to higher rates of misperceptions in (self-reported) hallucinating PD patients.

Although there has been considerable progress in recent years, visual misperceptions and hallucinations in PD remain poorly understood and are difficult to track and to treat. Thus, the availability of objective and quantifiable measures is needed to improve differential diagnosis, identification of risk groups, disease prognosis and treatment options.

The aim of the present study was to derive a quantifiable trial-based measure to investigate misperceptions and intra-individual variability and relate those behavioral markers to resting state functional connectivity. To our knowledge, intra-individual variability and its neuronal signature has not been investigated as a potential marker of visual misperceptions/hallucinations in PD, although intra-individual performance fluctuations have been described as sensitive markers to detect subtle cognitive deficits in a wide range of psychiatric and neurological diseases such as autism, head injury and dementia (MacDonald, Li, \& Bäckman, 2009). Previous research in schizophrenia and drug-induced psychosis also suggests a direct link to hallucinations (Fassbender, Lesh, Ursu, \& Salo, 2015; Fassbender, Scangos, Lesh, \& Carter, 2014; Rentrop et al., 2010). Cognitive fluctuations, which might be reflected in task performance variability, 
are related to desynchronization of fronto-parietal networks in patients with Lewy Body Dementia which is characterized by hallucinations (Peraza et al., 2014).

Based on previous research showing increased occurrence of visual misperceptions as measured in psychophysical tasks in PD patients with self-reported hallucinations (Koerts et al., 2010; Meppelink et al., 2008; Shine et al., 2012; Shine, Keogh, et al., 2015; Shine, Muller, et al., 2015), we employed a dynamic image recognition paradigm where image contrast was stepped up and subjects reported the detection of a face or car image while rejecting a scrambled version. Since it is unclear how conscious image perception contributes to the sensitivity of behavioral markers of $\mathrm{VH}$, we also implemented a Continuous Flash Suppression condition (CFS) to reduce perceptual awareness (Tsuchiya \& Koch, 2005). We calculated perceptual error scores and recognition time variability and correlated those outcome parameters to resting state functional connectivity as assessed with functional magnetic resonance imaging (fMRI). We tested the following hypotheses: 1) PD patients with self-reported visual hallucinations will make more perceptual errors such as reporting scrambled images as real images, possibly more so when the images are further suppressed from conscious perception. 2) PD patients with self-reported hallucinations will show increased intraindividual variability in their task performance and 3) Perceptual error scores (PES) and intra-individual variability (CVRT) are associated with impaired functional connectivity within or between attention-related networks that involve prefrontal cortices. 


\subsection{Materials and Methods}

\subsubsection{Participants}

Thirty-two patients with Parkinson's disease (16 PD patients without self-reported hallucinations (PD-nonVH) and 16 with self-reported hallucinations (PD-VH)) and 25 healthy age matched controls without a history of neurological or psychiatric diseases (assessed via questionnaire) were recruited. Exclusion criteria were moderate to severe general cognitive impairment/dementia (Mini Mental State Examination (MMSE) score < 26), visual acuity below $80 \%$ (corrected with glasses as necessary) and psychiatric disorders other than minor depression. We matched the groups based on demographic variables such as age, gender and years of post-secondary education (Table 2.1.). Diagnosis of idiopathic Parkinson's disease was made according to the UK Parkinson's Disease Society Brain Bank criteria by experienced neurologists. The study was approved by the medical ethics committees of the University Medical Center Goettingen, Germany.

Healthy controls and PD patients as a whole group did not differ in age $(p=0.17)$, gender $(p=0.48)$, education levels ranging from 0 (elementary school not finished) to 5 $(\mathrm{PhD})(\mathrm{p}=0.59)$ and years of post-secondary education $(\mathrm{p}=0.76)$. Mann-Whitney-Test was used for measurements which violated the normality assumption. Compared to healthy controls PD patients tended to have lower MMSE scores $(p=0.06)$. Consistent with previous research PD patients had increased Beck's Depression Inventory (BDI) scores $(p=0.00005)$ (Kritzinger et al., 2015) and lower contrast sensitivity $(p=0.009)$ (Meppelink et al., 2009) compared to healthy controls. 
PD-VH and PD-nonVH did not differ in age $(p=0.89)$, gender $(p=0.69)$, education levels $(p=0.76)$, years of post-secondary education $(p=0.47)$, MMSE scores $(p=$ $0.25)$, depression (BDI) scores $(p=0.45)$, contrast sensitivity $(U=86, p=0.11)$ and Hoehn \& Yahr stage $(p=0.10)$. Consistent with co-morbidity studies, PD-VH as compared to PD-nonVH patients had significantly longer disease durations $(p=0.007)$ and higher levodopa-equivalent daily dose (LEDD) $(p=0.0004)$ (Gupta, Singh, Khwaja, \& Mehndiratta, 2004) (Table 2.1.).

Table 2.1. Demographic and neuropsychological characteristics of volunteers that participated in the image recognition task

\begin{tabular}{|c|c|c|c|c|c|c|}
\hline & $\mathrm{HC}$ & PD (all) & & PD-VH & \multicolumn{2}{|c|}{ P-value } \\
\hline & $\begin{array}{l}\text { Mean } \\
\text { (SD) }\end{array}$ & $\begin{array}{l}\text { Mean } \\
\text { (SD) }\end{array}$ & $\begin{array}{l}\text { Mean } \\
\text { (SD) }\end{array}$ & Mean (SD) & HC vs. PD & $\begin{array}{l}\text { PD-VH } \\
\text { vs. PD- } \\
\text { nonVH }\end{array}$ \\
\hline Age (years) ${ }^{\mathrm{a}}$ & $\begin{array}{l}68.24 \\
(4.67)\end{array}$ & $\begin{array}{l}70.34 \\
(6.36)\end{array}$ & $\begin{array}{l}70.19 \\
(6.92)\end{array}$ & $\begin{array}{l}70.50 \\
(5.92)\end{array}$ & $=0.17$ & $=0.89$ \\
\hline $\begin{array}{c}\text { Gender (female/male) }{ }^{\mathrm{c}} \text {, } \\
\mathrm{n}(\%)\end{array}$ & $\begin{array}{l}5(20 \%) / \\
20(80 \%)\end{array}$ & $\begin{array}{l}9(28 \%) / \\
23(72 \%)\end{array}$ & $\begin{array}{l}4(25 \%) / \\
12(75 \%)\end{array}$ & $\begin{array}{l}5(31 \%) / \\
11(69 \%)\end{array}$ & $=0.48$ & $=0.69$ \\
\hline $\begin{array}{c}\text { Years of postsecondary } \\
\text { education }^{\text {b }}\end{array}$ & $\begin{array}{c}3.82 \\
(2.35)\end{array}$ & $\begin{array}{l}3.56 \\
(2.10)\end{array}$ & $\begin{array}{l}3.59 \\
(2.09)\end{array}$ & $\begin{array}{c}3.53 \\
(2.17)\end{array}$ & $=0.76$ & $=0.47$ \\
\hline MMSE $^{\mathrm{D}}$ & $\begin{array}{l}29.16 \\
(1.07)\end{array}$ & $\begin{array}{l}28.59 \\
(1.21)\end{array}$ & $\begin{array}{l}28.87 \\
(1.03)\end{array}$ & $\begin{array}{l}28.31 \\
(1.35)\end{array}$ & 0.06 & $=0.25$ \\
\hline Hoehn and Yahr stage $^{\mathrm{b}}$ & - & $\begin{array}{l}2.13 \\
(0.81)\end{array}$ & $\begin{array}{c}1.88 \\
(0.79)\end{array}$ & $\begin{array}{l}2.38 \\
(0.79)\end{array}$ & - & $=0.10$ \\
\hline Disease duration $^{b}$ & - & $\begin{array}{c}7.20 \\
(6.22) \\
\end{array}$ & $\begin{array}{c}4.61 \\
(3.68)\end{array}$ & $\begin{array}{c}9.78 \\
(7.22)\end{array}$ & - & $\begin{array}{c}= \\
0.007^{*}\end{array}$ \\
\hline UPDRS III & - & $\begin{array}{l}23.78 \\
(9.97)\end{array}$ & $\begin{array}{c}20.94 \\
(10.64)\end{array}$ & $\begin{array}{l}26.63 \\
(8.67)\end{array}$ & - & $=0.11$ \\
\hline $\mathrm{LEDD}^{\mathrm{a}}$, mg=day & - & $\begin{array}{c}591.76 \\
(353.84) \\
\end{array}$ & $\begin{array}{c}385.53 \\
(253.42)\end{array}$ & $\begin{array}{c}797.98 \\
(322.19)\end{array}$ & - & $\begin{array}{c}= \\
0.0004^{*}\end{array}$ \\
\hline UM-PDHQ & - & - & - & $9.13(2.53)$ & - & - \\
\hline $\mathrm{BDI}^{\mathrm{b}}$ & $\begin{array}{l}3.16 \\
(2.70)\end{array}$ & $\begin{array}{c}8.44 \\
(5.55)\end{array}$ & $\begin{array}{c}7.75 \\
(5.50)\end{array}$ & $\begin{array}{c}9.13 \\
(5.69)\end{array}$ & $=0.00005^{\dagger}$ & $=0.45$ \\
\hline Visual acuity (\%) & $\begin{array}{l}97.20 \\
(6.78) \\
\end{array}$ & $\begin{array}{l}93.75 \\
(9.42) \\
\end{array}$ & $\begin{array}{l}95.00 \\
(8.94) \\
\end{array}$ & $\begin{array}{c}92.50 \\
(10.00)\end{array}$ & $=0.16$ & $=0.45$ \\
\hline $\begin{array}{l}\text { Mars Letters Contrast } \\
\text { Sensitivity Test }^{b}\end{array}$ & $\begin{array}{c}1.76 \\
(0.05)\end{array}$ & $\begin{array}{c}1.71 \\
(0.09)\end{array}$ & $\begin{array}{c}1.74 \\
(0.08)\end{array}$ & $\begin{array}{c}1.68 \\
(0.10)\end{array}$ & $=0.009^{\top}$ & $=0.11$ \\
\hline
\end{tabular}

${ }^{a}$ t-test; ${ }^{b}$ Mann-Whitney $U$ test; BDI, Beck's, Depression Inventory; ${ }^{\circ}$ Chi-square -test; HC, healthy controls;LEDD, levodopa-equivalent daily dose; MMSE, Mini Mental State Examination; PD, Parkinson patients; PD-nonVH, Parkinson patients without visual hallucinations; PD-VH, Parkinson patients with visual hallucinations; UM-PDHQ, University of Miami Parkinson's disease Hallucinations Questionnaire; 
UPDRS, Unified Parkinson's Disease Rating Scale; †HC vs. PD $p<0.05$; *PD-nonVH vs. PD-VH $p<0.05$

Apart from 1 non-medicated PD-nonVH, all PD patients were tested under stable dopaminergic medication, 7 patients (4 PD-VH, 3 PD-nonVH) were taking antidepressants, and 4 were using opioids (2 PD-VH, 2 PD-nonVH). The levodopaequivalent daily dose (LEDD) was calculated for all patients, according to the formula described in the paper by Tomlinson (Tomlinson et al., 2010). In the PD-VH group, 44\% $(\mathrm{N}=7)$ reported visual hallucinations multiple times per day, $25 \%(\mathrm{~N}=4)$ multiple times per week, and $31 \%(\mathrm{~N}=5)$ less than weekly. PD-VH reported complex visual hallucinations such as people (63\%), animals (50\%), insects $(25 \%)$, objects $(38 \%)$ and simple visual hallucinations (6\%). Five (31\%) PD-VH also reported auditory hallucinations.

A sample of 16 (10 PD-nonVH, 6 PD-VH) Parkinson patients and 19 age-matched healthy control subjects participated in a resting-state scan ( $6 \mathrm{~min})$, performed either on the same or one to eight days after the behavioral experiment. Demographic and clinical variables of the fMRI sample are shown in Supplementary Table S2.1. In short, HC and PD patients did not differ with respect to age $(p=0.66)$, education level $(p=$ $0.69)$, years of post-secondary education $(p=0.88)$, contrast sensitivity $(p=0.78)$. PD patients had higher depression (BDI) scores $(p=0.02)$ and tended to have lower cognitive (MMSE) scores $(p=0.06)$.

\subsubsection{Clinical Test Batteries}

Motor symptoms were assessed with the Unified Parkinson Disease Rating Scale (UPDRS), part III (Goetz et al., 2008). General cognitive performance was tested with 
the Mini Mental State Examination (MMSE) (Folstein, Folstein, \& McHugh, 1975). Participants filled in a self-report depression scale (Beck's Depression Inventory, BDI) (Beck, Ward, Mendelson, Mock, \& Erbaugh, 1961). Severity of hallucinations was assessed by a structured interview based on the hallucinations questionnaire (University of Miami Parkinson's disease Hallucinations Questionnaire (UM-PDHQ)) (Papapetropoulos et al., 2008). Close vision test was performed to assess visual acuity (Poster Werner Radler). Contrast sensitivity was evaluated with the Mars Letters Test (Mars Perceptrix Corporation, Chappaqua, NY, USA).

\subsubsection{Experimental Design and Procedures}

\subsubsection{Control task with unmasked images at $100 \%$ luminance contrast}

Before the start of the main experiment, participants were presented with 20 car or face images at $100 \%$ contrast. Subjects were instructed to press a pre-assigned button according to picture category. Maximal response time was 12 seconds.

\subsubsection{Main perceptual task}

Participants were sitting in a dimly lit room in front of an LCD monitor. Target stimuli consisted of photographs of faces (Ekman \& Friesen, 1976), cars (http://vision.caltech.edu/archive.html) or their scrambled versions, presented in a pseudorandom order (Figure 2.1, further details in Supplementary Materials). 
A

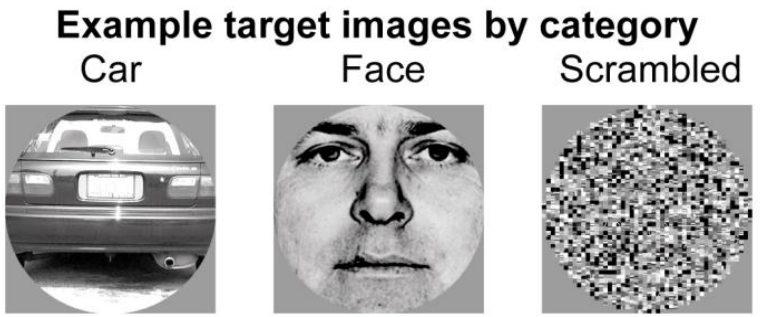

B

\section{Unmasked Target (non-CFS)}

\section{Dichoptic Masking (CFS)}

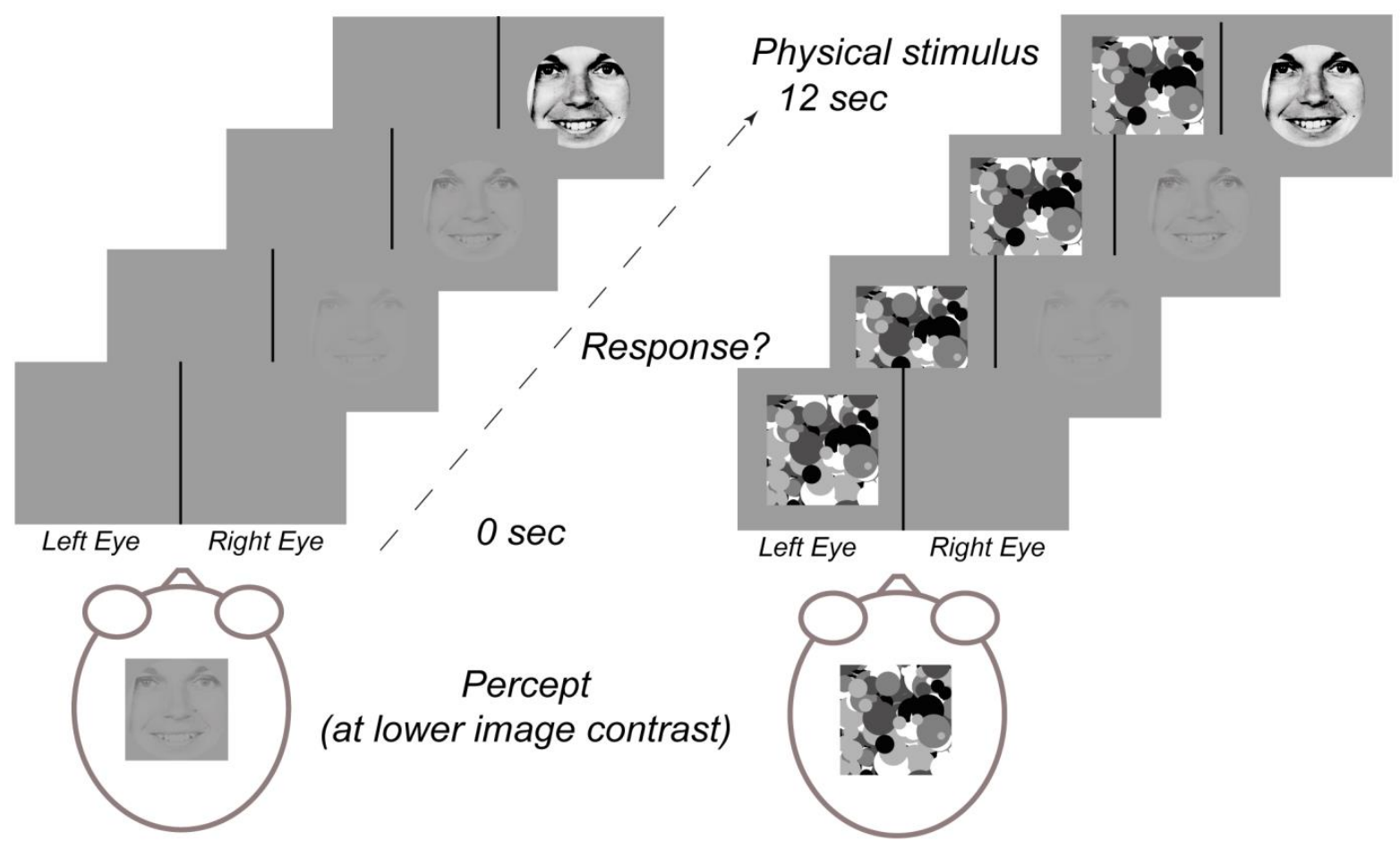

Figure 2.1. Task design and trial structure. A) Example images of face, car and scrambled images used in the experiments. Trial structure B) non-CFS and C) CFS condition.

Each trial started with the presentation of a central fixation cross, followed by the onset of the target image 600 ms later. Subjects were asked to maintain central fixation and to avoid eye blinks throughout the trial. Image luminance contrast was continuously stepped up over 10 seconds. Subjects were instructed to press a pre-assigned button 
as quickly as possible when they detected a target image and to not press any button when the image category was not recognized or when a scrambled image was shown. A trial ended either after the response was given, or at 12 seconds after image onset (Figure 2.1B-C).

We created a 'visible' (Figure 2.1B) and an 'invisible' condition (Figure 2.1C). For the 'invisible' condition we used continuous flash suppression (CFS), designed to suppress the images from consciousness for several seconds via the presentation of high contrast and rapidly changing Mondrian patterns to the eye opposite of the target image (Tsuchiya \& Koch, 2005) (Figure 2.1C). Non-CFS and CFS trials were presented in a pseudorandomized order. Since the CFS condition did not provide additional insights into differences between hallucinating and non-hallucinating PD patients (i.e. group effects were smaller) we primarily focus the presentation of the results on the non-CFS condition as depicted in Figure 2.1B. The non-CFS condition consisted of 100-120 trials per subject ( $1 / 3$ of each image category).

\subsubsection{Resting state image acquisition}

Subjects were instructed to stay awake with their eyes closed and not to think of anything in particular during the resting-state scan ( 6 min.) $\mathrm{fMRI}$ data were acquired using a 3 Tesla MR system (Magnetom TIM Trio, Siemens Healthcare, Erlangen, Germany) with a 32-channel phased-array head coil. fMRI resting-state experiments were performed using the 2D multiband gradient-echo planar imaging sequence from the Center for Magnetic Resonance Research, University of Minnesota (Moeller et al., 2010; Setsompop et al., 2012) with $\mathrm{T}^{*}$-weighting at $3 \times 3 \mathrm{~mm}^{2}$ in-plane resolution, oriented parallel to the AC-PC plane (TR: $1800 \mathrm{~ms}$, TE: $30 \mathrm{~ms}$, flip angle: $70^{\circ}, 34$ slices 
of $3 \mathrm{~mm}$ thickness with $20 \%$ inter-slice gap, field-of-view: $192 \mathrm{~mm} \times 192 \mathrm{~mm}$, matrix size: $64 \times 64$, multiband acceleration factor 3, 194 whole-brain volumes per functional run).

\subsubsection{Analyses}

\subsubsection{Demographic, clinical and behavioral statistical data analysis}

Data analysis was performed using custom written scripts in MATLAB R2012b and SPSS (version 24; SPSS, Inc., Chicago, IL). Univariate ANOVA and t-tests were used to compare age, UPDRS-III and LEDD. Chi-square test was used to compare gender. Non-parametric Mann-Whitney U-Tests were used to compare years of post-secondary education, education level, years of disease duration, Hoehn and Yahr stage, BDI and MMSE scores, visual acuity and contrast perception between groups.

Unless otherwise noted, behavioral analysis was performed by a mixed ANOVA with the within-subject factor "Category" (faces vs. cars) and the between-subject factor "Group". Two types of ANOVAs were calculated: 1) ANOVA with the "Group" factor HC vs. all PD patients (PD-VH + PD-nonVH) together (in the following "ANOVA_1"). 2) To investigate behavioral markers that are specific for VH in PD we used a separate ANOVA with the "Group" factor PD-nonVH vs. PD-VH (in the following "ANOVA_2").

The description of the results will focus on main and interaction effects of "Group", additional ANOVA results are provided in (Table 2.3., Supplementary Table S2.2.). Bonferroni correction was applied for multiple comparisons of the main effect of factor "Group" in ANOVA_1 and ANOVA_2, resulting in a significance threshold of $p<0.025$. 


\section{Additional control analyses}

When there was a significant difference between PD-VH and PD-nonVH we performed an additional control analysis by an ANCOVA with levodopa daily dose and disease duration as covariates. The co-variate analyses were performed since disease duration and LEDD significantly differed between PD-nonVH and PD-VH.

To exclude possible influences of individual values on the group differences, reliability of the results for the main outcome measures, perceptual error score and coefficient of recognition time variability, was tested applying the jackknife procedure by repeating the patient comparison by systematically removing one sample and re-calculating the mean (Wilke, 2012).

\subsubsection{Key performance measures}

Performance parameters were calculated separately for each image category (faces or cars).

Image Recognition Performance

I) Proportion Correct Recognition: Proportion Correct Categorization (face reported as a face and car reported as car) divided by the overall number of image presentations including missed responses; II) Proportion Misses: Trials in which a face or a car was presented but the subject did not provide a button response divided by the overall number of image presentations; III) Proportion erroneous face or car reports in scrambled images; IV) Perceptual error score (PES): Proportion errors consisting of category confusions (face reported as car and vice versa), misses and false real image detection in scrambled images. 
Recognition times: means and intra-individual variability

Mean recognition times (RT) denote the time between image onset and button response. Only correct responses were used to compute mean RT. Coefficient of recognition time variability (CVRT) was calculated by dividing the standard deviation of individual recognition times for a given condition and image category (car, face) by the individual mean of recognition times (standard deviation/mean) (Flehmig, Steinborn, Langner, Scholz, \& Westhoff, 2007).

\subsubsection{Resting state functional connectivity}

\section{Preprocessing}

All fMRI data processing was performed using the CONN Toolbox (www.nitrc.org/projects/conn, RRID: SCR_009550). For each subject's data set, preprocessing steps included: functional motion estimation and correction, functional and structural data translations to center ( $0,0,0$ coordinates), functional data slice-timing correction, functional ART-based outlier detection, direct, simultaneous gray matter/white matter/cerebral spinal fluid (GM/WM/CSF, respectively) segmentation and MNI normalization applied to functional and structural data separately, and $6 \mathrm{~mm}$ fullwidth-at-half-maximum-kernel Gaussian smoothing. The following denoising steps were implemented: linear regression of confounding effects, including GM, WM, and CSF signals (CompCor method) (Behzadi, Restom, Liau, \& Liu, 2007), realignment parameters and their first derivatives, ART-detected outlier volumes, and the effect of the resting task (temporal filter reducing weight of initial 10 scans in the run). Linear detrending was applied prior to a $0.008-0.09 \mathrm{~Hz}$ bandpass filter. 
Statistical analyses: voxelwise volume analysis

Group-level independent component analysis (ICA) was applied to the whole sample of combined patients and healthy controls in order to spatially define resting state networks (RSNs). Data dimensions were reduced through retaining 40 components of an initial principle component analysis of the temporally-concatenated runs for the whole group, followed by 20 -component ICA. Of the 20 resulting components, eight were visually identified as RSNs of interest as previous imaging studies reported impairments in frontal, parietal and visual brain regions (Baggio et al., 2015; Prell, 2018): default mode (DMN), dorsal attention (DAN), left and right fronto-parietal (IFP, rFP, respectively), medial and lateral visual (medVIS and latVIS, respectively), somatomotor (SMN), and salience (SAL) (for more detailed network description see Table 2.2.). Dual regression was applied to the group ICA results using all components generated from the initial analysis, resulting in subject-level beta maps of all components, including the RSNs of interest (Beckmann, Mackay, Filippini, \& Smith, 2009). For second-level statistical tests, comparisons were made with network-level beta averages against behavioral statistics as well as equivalent full-volume voxelwise tests that included behavioral covariates. To generate masks for subject-level RSN averages, for each of the selected components, a one-sample t-test was performed using all subjects, and an uncorrected voxel-level $p=0.05$ and FWE-corrected clusterlevel $p=0.05$ were applied. Threshold values were chosen liberally to include each RSN without excessive overlap among networks. Figure 2.2. shows the resting state network maps from group-level ANOVA results of the ICA analysis. Resulting thresholded maps were binarized to be used as RSN masks to summarize within- and 
between-network functional connectivity for group-level statistics. For within- and between-network functional connectivity measures, for every subject, each group-level RSN mask was applied to each network volume map to extract average beta values.

Subject-level within- and between-network average beta values were used in further analysis with behavioral data in SPSS.

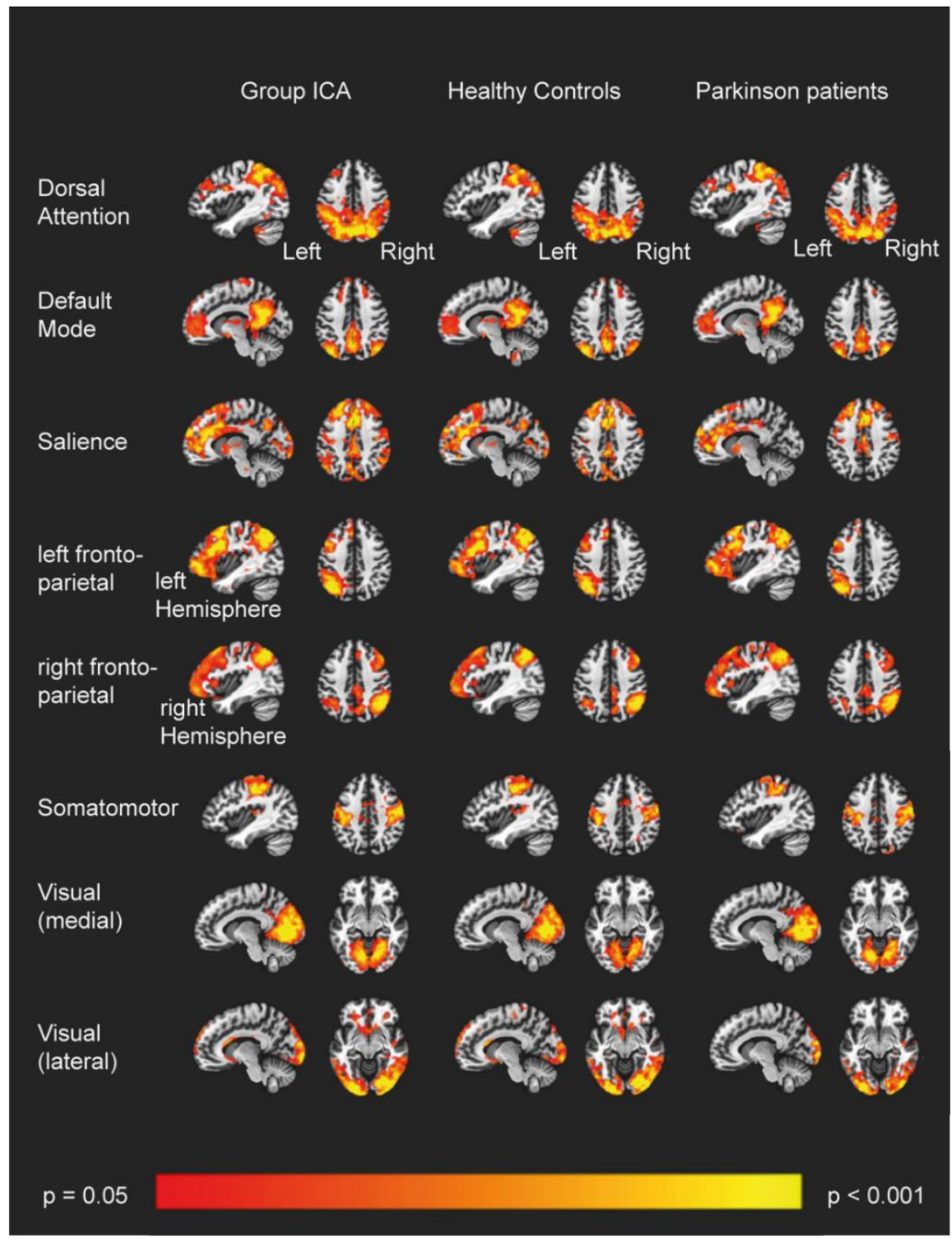

Figure 2.2. ICA Resting state networks. Spatial maps representing the eight resting state networks of interest, generated through ANOVAs on each chosen ICA result, displaying the simple main effect of 
each RSN component for all subjects (left), healthy controls (center), and all PD patients (right; for all maps: uncorrected voxel-level $p<0.05$, FWE-corrected cluster $p<0.05)$.

Table 2.2. Overview of Networks of Interest.

\begin{tabular}{|c|c|}
\hline Network & Brain areas \\
\hline Dorsal Attention & $\begin{array}{l}\text { Precuneus, Inferior and Superior Parietal Lobule, Pre- and Postcentral Gyrus } \\
\text { Cuneus, Paracentral Lobule, Middle and Superior Occipital Gyrus } \\
\text { Middle and Superior Frontal Gyrus, Fusiform Gyrus, Inferior Temporal Gyrus }\end{array}$ \\
\hline Default Mode & $\begin{array}{l}\text { Precuneus, Posterior Cingulate, Inferior, Middle and Superior Temporal Gyrus } \\
\text { Cuneus, Parahippocampal Gyrus, Angular Gyrus, Superior Occipital Gyrus } \\
\text { Inferior Parietal Lobule, Anterior Cingulate, Fusiform Gyrus, Right Insula } \\
\text { Middle and Superior Frontal Gyrus }\end{array}$ \\
\hline Salience & $\begin{array}{l}\text { Medial and Superior Frontal Gyrus, Cingulate Gyrus, Anterior Cingulate } \\
\text { Precuneus, Superior Temporal, Insula, Pre- and Postcentral Gyrus, } \\
\text { Lingual Gyrus, Cuneus, Middle Occipital Gyrus, Lentiform Nucleus } \\
\text { Inferior Parietal Lobule }\end{array}$ \\
\hline Left fronto-parietal & $\begin{array}{l}\text { Left Inferior, Middle and Superior Frontal Gyrus, Left Precentral Gyrus } \\
\text { Left Middle and Superior Temporal Gyrus, Left Inferior and Superior Parietal } \\
\text { Lobule, Left Precuneus, Left Supramarginal Gyrus }\end{array}$ \\
\hline $\begin{array}{l}\text { Right fronto- } \\
\text { parietal }\end{array}$ & $\begin{array}{l}\text { Right Inferior, Middle and Superior Frontal Gyrus, Right Precentral Gyrus } \\
\text { Right Inferior and Superior Parietal Lobule, Right Precuneus } \\
\text { Right Angular Gyrus, Right Superior Temporal Gyrus }\end{array}$ \\
\hline Somatomotor & $\begin{array}{l}\text { Medial and Superior Frontal Gyrus, Post- and Precentral Gyrus } \\
\text { Paracentral Lobule }\end{array}$ \\
\hline Visual lateral & Inferior, Middle and Superior Occipital Gyrus, Cuneus, Fusiform Gyrus \\
\hline Visual medial & Cuneus, Lingual Gyrus, Precuneus \\
\hline
\end{tabular}

\subsubsection{Group differences in Resting state networks}

To investigate differences between $\mathrm{HC}$ and $\mathrm{PD}$ patients we performed full-volume voxelwise comparisons for the eight networks of interest (default mode, dorsal attention, salience, right and left fronto-parietal, somatomotor, visual medial and lateral). To test differences of within network connectivity, averages of the eight networks were submitted to a univariate ANOVA, with "Group" (HC and PD) as a between subject factor. Given the limited sample size (PD-nonVH = 10, PD-VH =6) we did not compare the PD patient subgroups. 


\subsubsection{Correlation between Resting state functional connectivity and Perceptual Error Score (PES) and Coefficient of intra-individual variability (CVRT)}

In order to investigate the relationship between functional connectivity averages and the behavioral markers PES and CVRT, we performed for the PD group Spearman's rho correlations. Partial correlations of PD patients including disease duration and daily levodopa dose (LEDD) were used as control variables (Baggio et al., 2015; Shine, Keogh, et al., 2015). Given the limited subsample size, we did not separate the PD patient groups when correlating the functional connectivity estimates with the main outcomes measures. PES scores violated the assumptions of linear correlation and were thus transformed using logarithmic transformation (log (PES + 1)) (Bartlett, 1947; Maria et al., 2003; Morrison, Wells, \& Nothard, 2000). 


\subsection{Results}

\subsubsection{Correct image recognition in the control task}

In the control task in which either face or car images were presented at $100 \%$ contrast, the proportion of correctly categorized face and car images did not significantly differ between healthy controls and PD patients (ANOVA_1: $F(1,55)=0.0001, p=0.99$ ) nor between the two PD patient groups (ANOVA_2: PD-nonVH vs. PD-VH: $(F(1,30)=0, p=$ 1). In all three groups the proportion correct image recognition in the control task was above 95\% (HC: 97.2\%, PD-nonVH: 97.19\%, PD-VH: 97.19\%) verifying that all groups were able to perform the task.

\subsubsection{Correct image recognition in the main task (non-CFS)}

Figure 2.3A shows that $\mathrm{HC}$ correctly recognized the images in $99 \%$ while PD patients reached on average only 90\%. Accordingly, the ANOVA_1 showed a main group effect between $\mathrm{HC}$ and PD patients $(F(1,55)=9.52, \mathrm{p}=0.003)$.

The separate comparison between the two PD patient groups showed that PD-VH patients recognized fewer images correctly than the PD-nonVH, expressed as a significant group effect in the ANOVA_2, (ANOVA_2: $F(1,30)=11.42, p=0.002$ ) (Figure 2.3A, right column). Given the larger levodopa daily dose (LEDD) and longer disease durations of PD-VH patients as compared to PD-nonVH, we conducted an additional analysis including and disease duration as covariates in the ANCOVA. This control analysis did not yield a significant difference in respect with correct image recognition between the two PD groups $(F(1,30)=1.96, p=0.17)$, indicating that dopaminergic medication and/or disease duration might be confounds explaining the 
lower recognition performance in $\mathrm{PD}-\mathrm{VH}$ patients.

With respect to misses (i.e. no indicated image recognition within the 12 seconds of a trial), PD patients as a group had a non-significant tendency for more misses (Figure 2.3B). The comparison between the two PD groups yielded a tendency for a higher proportion of misses in the PD-VH group (ANOVA_2: PD-VH: 9\%, PD-nonVH: 0.16\%, (main effect of "Group": $F(1,30)=5.12, p=0.03)$, albeit not passing the Bonferroni correction $(p<0.025)$. 


\section{A Proportion Correct Image Recognition}
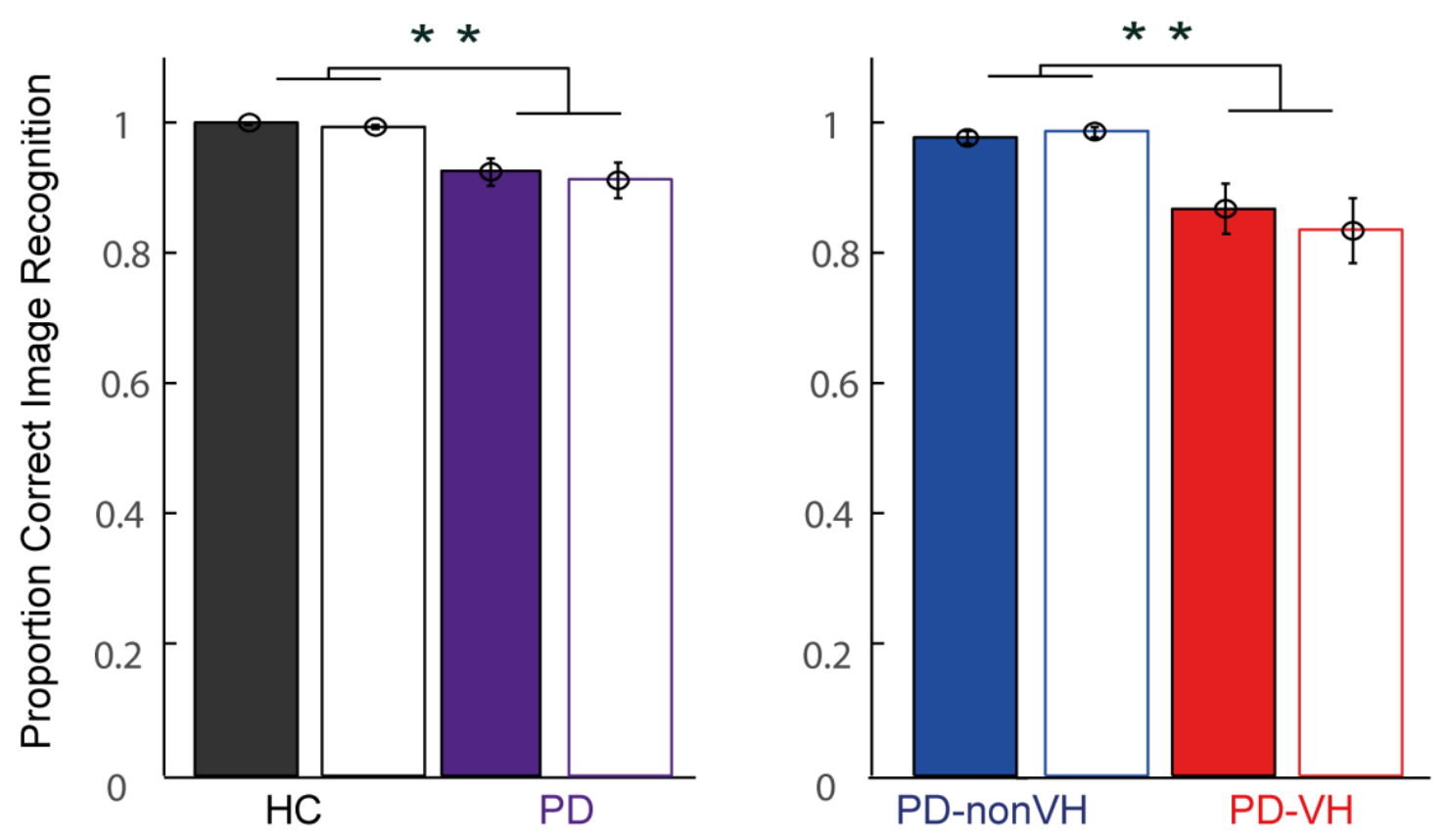

B

Proportion Misses

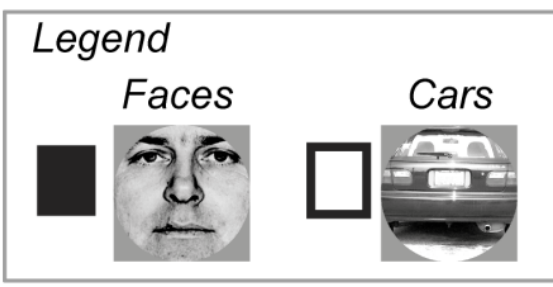

$\square \mathrm{HC}$

PD

PD-nonVH

PD-VH
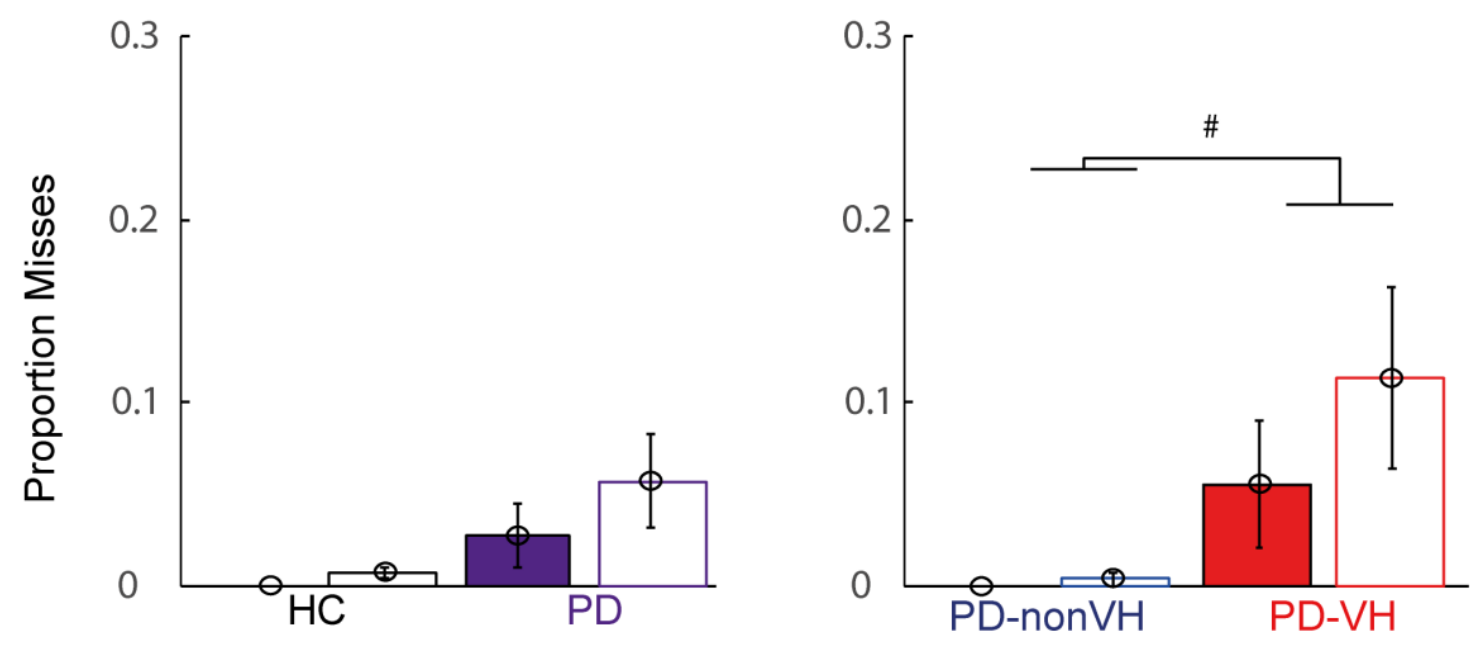

Figure 2.3. Proportion Correct Image Recognition and Misses. (A) Mean proportion of correct image recognition of faces and cars. (left) $\mathrm{HC}(\mathrm{N}=25)$ vs. $\mathrm{PD}(\mathrm{N}=32)$ (right) PD-nonVH $(\mathrm{N}=16)$ vs. PD-VH ( $\mathrm{N}$ 
= 16) (B) Mean proportion of missed faces and cars. (left) $\mathrm{HC}(\mathrm{N}=25)$ vs. $\mathrm{PD}(\mathrm{N}=32)$ (right) PD-nonVH $(\mathrm{N}=16)$ vs. PD-VH $(\mathrm{N}=16)$. In $(\mathrm{A})$ and $(\mathrm{B}){ }^{* *}$ denotes a significant group difference, $\mathrm{p}<0.005$ and \# denotes $p<0.05$ without Bonferroni correction as assessed by ANOVA_1 (HC vs. PD) and ANOVA_2 (PD_nonVH vs. PD_VH). Error bars denote S.E.M. across subjects.

\subsubsection{Erroneous detection of real life images in scrambled images (non-CFS)}

Figure 2.4. shows that PD patients tended to report real images in scrambled images more often than $\mathrm{HC}$ (HC: $6 \%$, PD: $20 \%$, ANOVA_1: $F(1,55=4.86, p=0.03$, n.s. after Bonferroni correction). The ANOVA comparing PD-nonVH with PD-VH revealed that this effect was mainly driven by the PD-VH, who reported a real image in $32 \%$ of trials as compared to PD-nonVH with only $8 \%(F(1,30)=7.56, p=0.01)$ (Figure 2.4., right column). The higher proportion of false image reports in the PD-VH group is also supported by a significant effect of "Group" $(F(1,28)=12.75, p=0.001)$ in the ANCOVA control analysis using LEDD and disease duration as covariates. 
Healthy controls $\square$ PD-all $\square$ PD-nonVH $\quad$ PD-VH
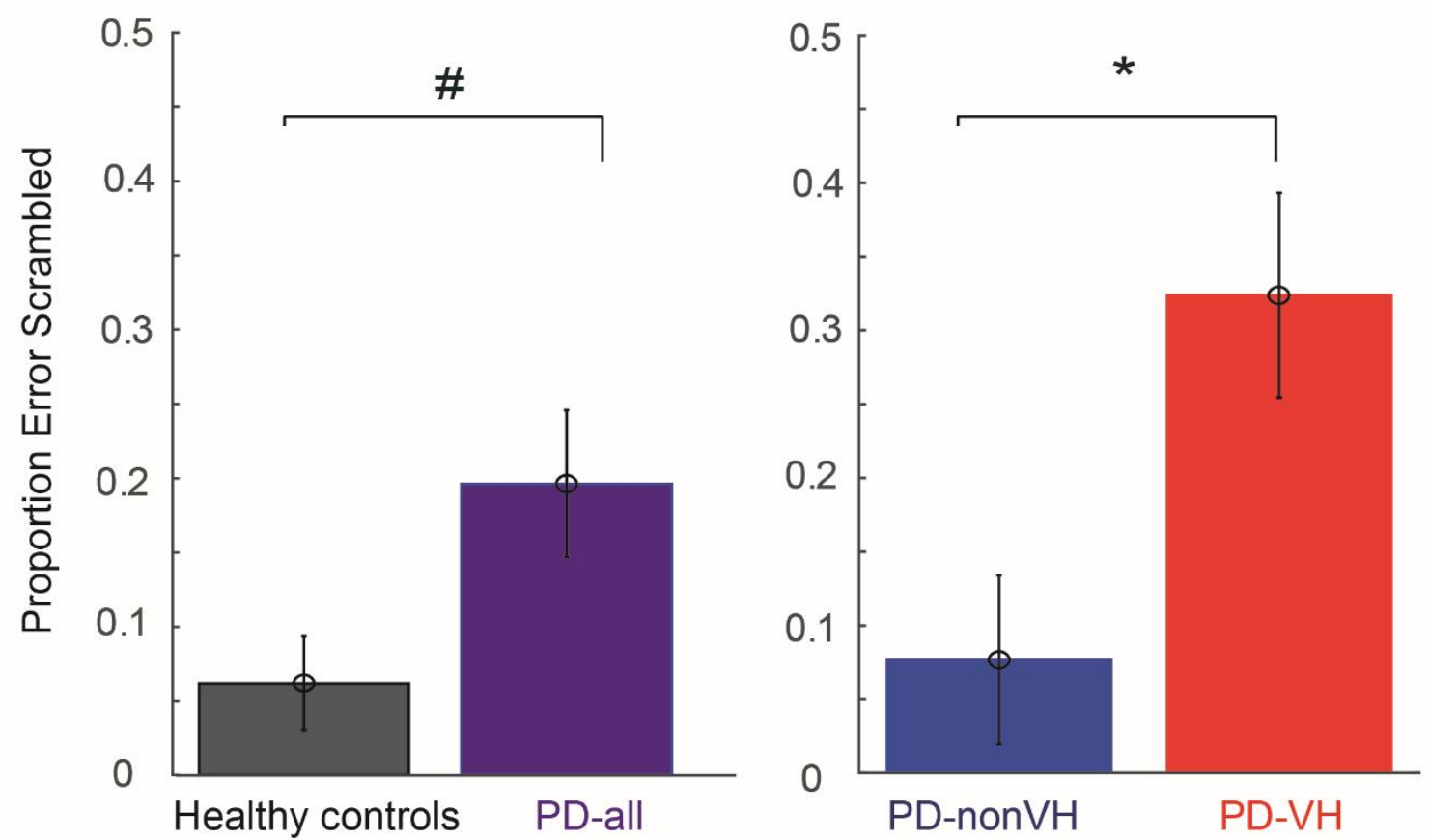

Figure 2.4. Erroneous Image Recognition in Scrambled Images. Mean proportion of erroneous image recognition in scrambled images. (left) $\mathrm{HC}(\mathrm{N}=25)$ vs. PD $(\mathrm{N}=32)$ (right) PD-nonVH $(\mathrm{N}=16)$ vs. PD-VH $(\mathrm{N}=16){ }^{*}$ denotes a significant group difference (Bonferroni correction, $\mathrm{p}<0.025$ ) as assessed by ANOVA_2 (PD_nonVH vs. PD_VH). \# denotes a significant group difference (without Bonferroni correction, $p<0.05$ ) as assessed by ANOVA_1 (HC vs. PD). Error bars denote S.E.M. across subjects.

\subsubsection{Perceptual Error Score (non-CFS)}

Assuming that a combined sum score of different image recognition errors is more robust in clinical studies than the separate ones, we computed a sum perceptual error score (PES) of category confusion, misses and false real image detection in scrambled images. As illustrated in Figure 2.5., PD patients (14\%) had higher PES scores 
compared to $\mathrm{HC}(3 \%)$ which is also revealed by the main effect of "Group" in the ANOVA (ANOVA_1: $F(1,55)=9.81, p=0.003)$.

\section{Perceptual Error Score}

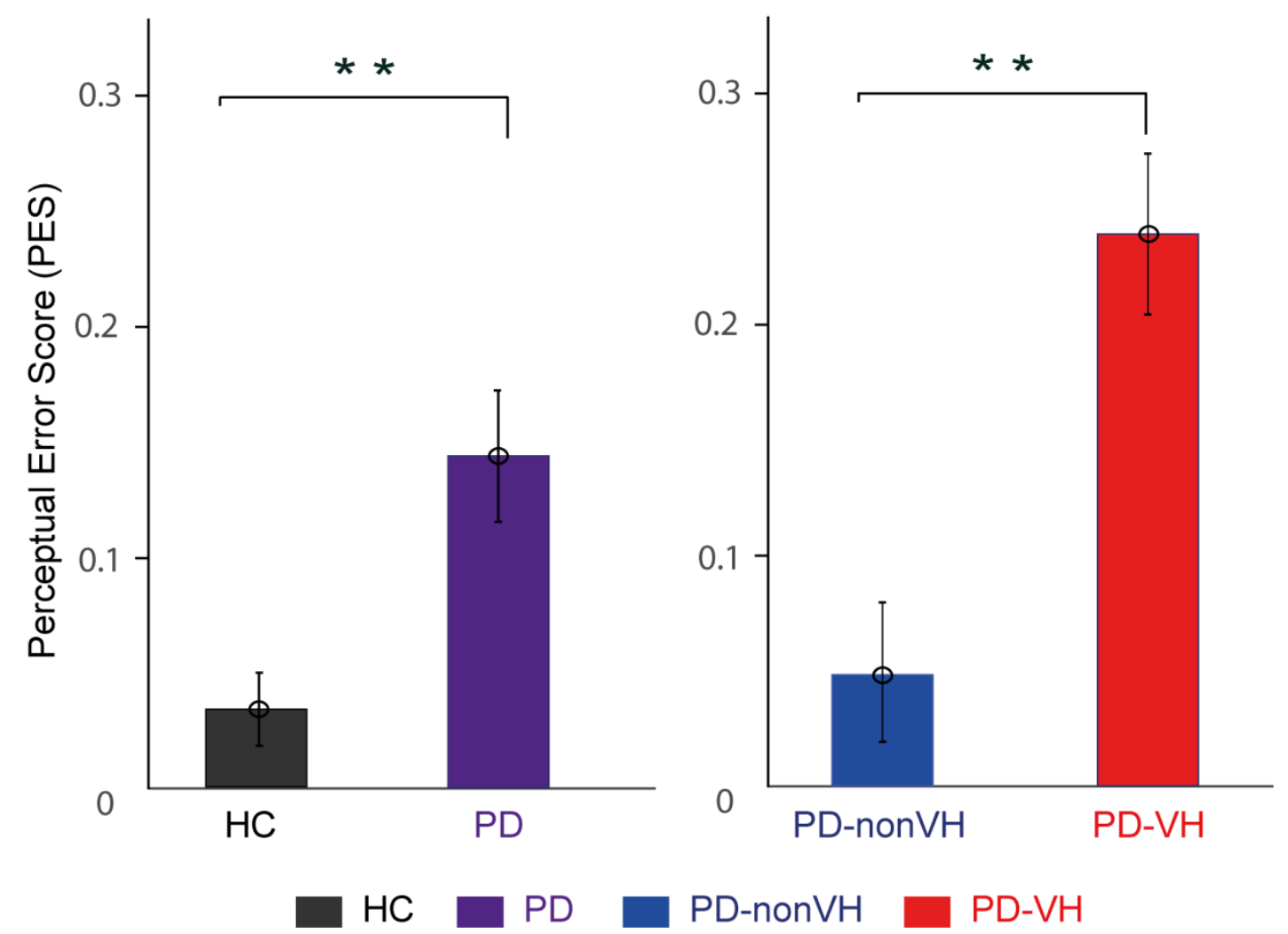

Figure 2.5. Perceptual Error Score (PES). Mean Perceptual Error Score, separated by subject group. (left) $\mathrm{HC}(\mathrm{N}=25)$ vs. $\mathrm{PD}(\mathrm{N}=32)$ (right) $\mathrm{PD}-n o n V H(\mathrm{~N}=16)$ vs. PD-VH $(\mathrm{N}=16) .{ }^{* *}$ denotes a significant group difference, $p<0.005$ by ANOVA_1 (HC vs. PD) and ANOVA_2 (PD-nonVH vs. PD-VH). Error bars denote S.E.M. across subjects.

Figure 2.5. (right column) shows that this effect is led by the higher PES scores in PD$\mathrm{VH}(24 \%)$ compared to PD-nonVH (5\%), expressed also by the main effect of "Group" in the ANOVA comparing PD-nonVH and PD-VH (ANOVA_2: $F(1,30)=17.16, p=$ 0.0003). The ANCOVA comparing PD-nonVH with PD-VH that included levodopa daily 
dose and disease duration as covariates showed a robust effect, with a significant effect of "Group" $(F(1,28)=13.88, p=0.001)$. This suggests that the differences in PES cannot be explained only by the differences in LEDD and disease duration.

To further demonstrate the stability of the group difference in PES between PD-VH and PD-nonVH, a Jackknife procedure was applied. Systematically leaving out one subject from the analysis and recomputing the ANOVA_2 always resulted in significant main effects of "Group" (all $p$ values were in the range [0.0001 - 0.0005]). Finally, the calculation of PES cut-off scores underlines its potential to discriminate between PDnonVH and PD-VH. Specifically, 11 PD-VH patients (69\%) scored above the PES cut off score of 0.15 (average PES score of $\mathrm{HC}+1.5 \mathrm{SD}$ of $\mathrm{HC}$ ) as compared to only $1 \mathrm{PD}$ nonVH (6\%) and $2 \mathrm{HC}(8 \%)$, showing its potential discriminative value in detecting hallucinating PD patients.

\subsubsection{Mean Recognition times (non-CFS)}

As expected from previous studies (e.g. (Meppelink et al., 2008), PD patients were slower in image recognition than $\mathrm{HC}(F(1,55)=8.87, p=0.004)$. (Supplementary Table S2.2.). Comparing the two PD groups, PD-VH had longer mean recognition times than PD-nonVH $(F(1,30)=13.58, p=0.001)$ (Table 2.3.). When LEDD and disease duration were included in the ANCOVA, the two patient groups did not significantly differ in respect to mean recognition times $(F(1,28)=2.78, p=0.11)$, indicating that the LEDD and/or disease duration contributed to this recognition time effect (Supplementary Table S2.3.). 


\subsubsection{Intra-individual variability of recognition times (non-CFS)}

So far we considered only mean recognition times. However, intra-individual variability is increased in medicated and unmedicated PD patients and is discussed as a possible marker of ensuing cognitive dysfunction in PD (Camicioli et al., 2008; de Frias, Dixon, Fisher, \& Camicioli, 2007). In the next step we assessed the intra-individual variability of RTs expressed as the coefficient of individual recognition time variability across trials (CVRT) (MacDonald et al., 2009). As shown in Figure 6, the CVRT of the PD patients as a whole group, did not differ from the HC (ANOVA_1, $F(1,55)=0.47, p=0.5)$.

The direct comparison between the two PD groups showed a larger RT variability for faces and cars in the PD-VH (0.23) as compared to PD-nonVH (0.19), which is also expressed by the main effect of "Group" in the ANOVA (ANOVA_2, $F(1,30)=8.43, p=$ 0.007)(Figure2.6). 


\section{Individual Variability of RT (CVRT = i_std / i_mean)}
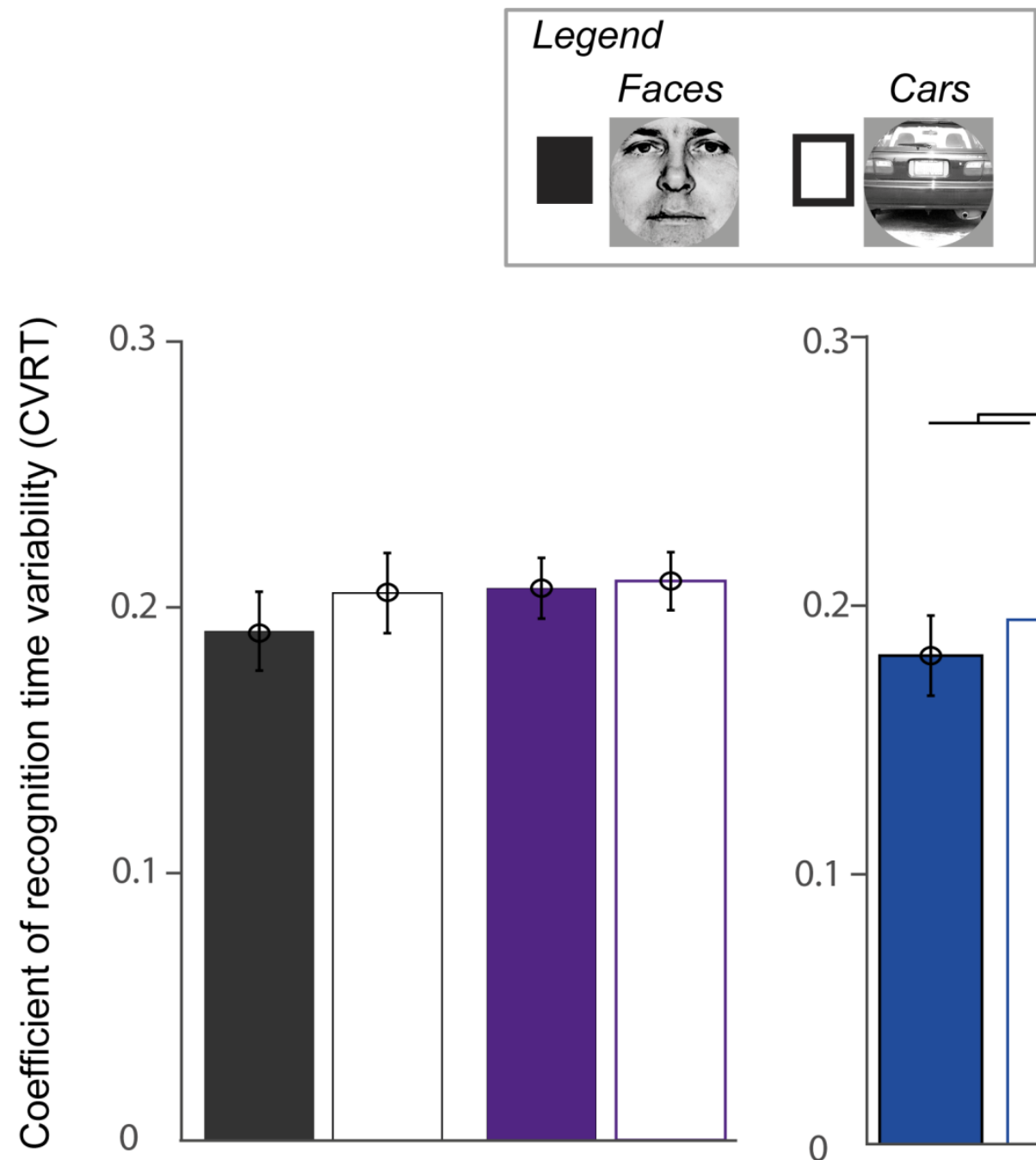

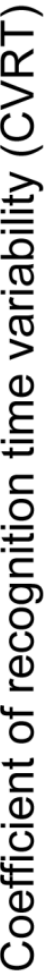
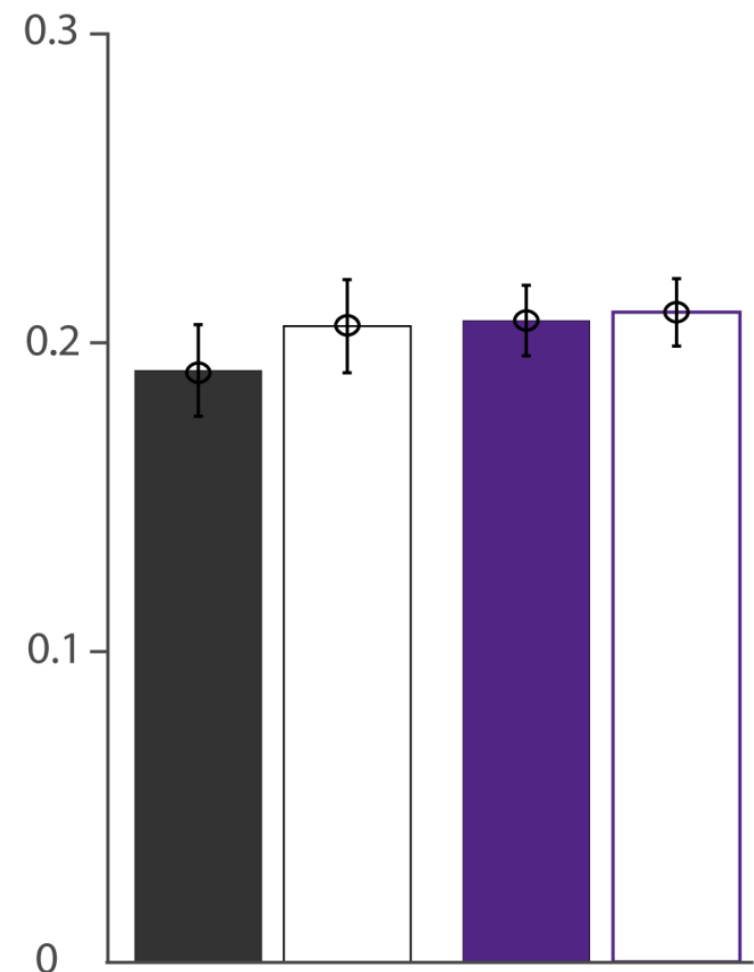

$\mathrm{HC}$

PD

0.37

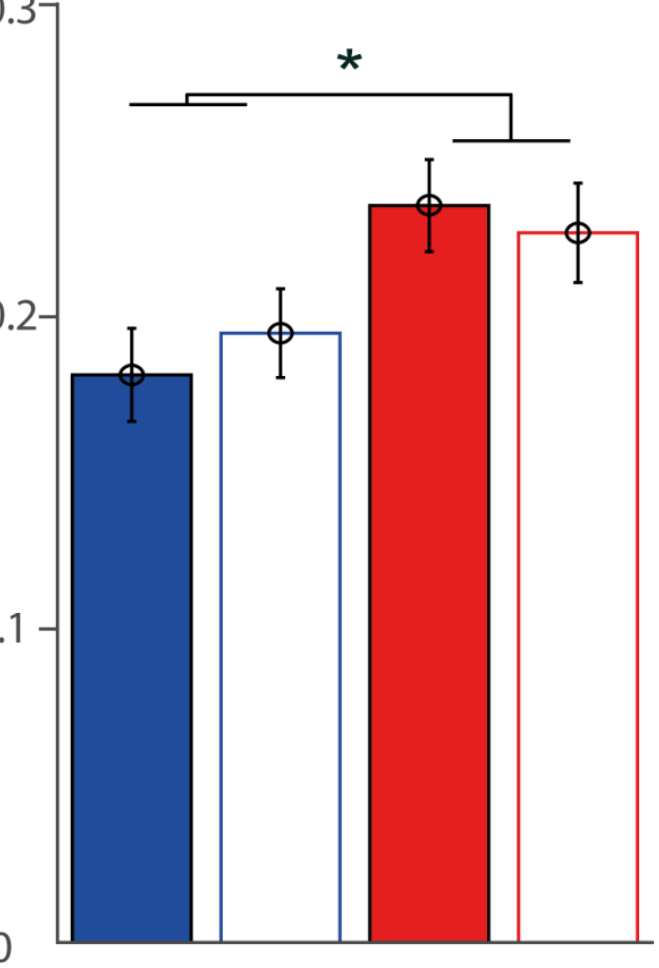

PD-nonVH PD-VH

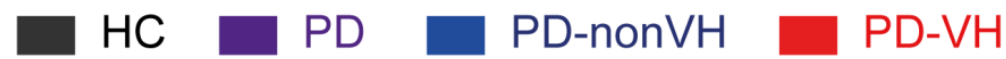

Figure 2.6. Coefficient of Recognition Time variability. Average of individual variability in RT (CVRT = individual standard deviation of RT divided by the individual RT mean). (left) No significant group differences between HC and PD as analyzed by ANOVA are shown. (right) PD-nonVH vs. PD-VH, * denotes a significant group difference, Bonferroni corrected for multiple comparisons $p<0.0025$ (black star: PD-nonVH vs. PD-VH as assessed by the ANOVA_2). 
The difference between PD-VH and PD-nonVH remained significant when including disease duration and LEDD as covariates in the ANCOVA $(F(1,28)=4.34, p=0.047)$ (Supplementary Table S2.3).

Supplementary Figures S2.1. and S2.2. illustrate that this effect cannot be explained by outliers or by an increase in RT during the experiment. To ensure the stability of this finding we performed the Jackknife procedure which showed reliability of the CVRT group differences of $p$-values in the significant range $[0.005 ; 0.01]$.

Table 2.3. ANOVA_2: Repeated Measures mixed ANOVA with the factors Category, Group and interaction effects for Parkinson patients without visual hallucinations (PD-nonVH) and Parkinson patients with visual hallucinations (PD-VH)

\begin{tabular}{|c|c|c|c|c|c|c|}
\hline Factor & $\begin{array}{l}\text { Proportion } \\
\text { Correct } \\
\text { Recognition }\end{array}$ & $\begin{array}{l}\text { Proportion } \\
\text { Misses }\end{array}$ & $\begin{array}{l}\text { Proportion } \\
\text { Erroneous } \\
\text { Object } \\
\text { Recogni } \\
\text { tion in } \\
\text { Scrambled } \\
\text { Images }\end{array}$ & $\begin{array}{l}\text { Perceptual } \\
\text { Error Score }\end{array}$ & CVRT & RT \\
\hline & $F(p)$ & $F(p)$ & $F(p)$ & $F(p)$ & $F(p)$ & $F(p)$ \\
\hline Category & $\begin{array}{l}1.74 \\
(=0.19)\end{array}$ & $\begin{array}{l}9.63 \\
(=0.004)^{\star *}\end{array}$ & & & $\begin{array}{l}0.01 \\
(=0.92)\end{array}$ & $\begin{array}{l}164.96 \\
(<0.0001)^{\star *}\end{array}$ \\
\hline Group & $\begin{array}{l}11.42 \\
(=0.002)^{\star \star}\end{array}$ & $\begin{array}{l}5.12 \\
(=0.03) \#\end{array}$ & $\begin{array}{l}7.56 \\
(=0.01)^{*}\end{array}$ & $\begin{array}{l}17.16 \\
(=0.0003)^{\star \star}\end{array}$ & $\begin{array}{l}8.43 \\
(=0.007)^{\star *}\end{array}$ & $\begin{array}{l}13.58 \\
(=0.001)^{\star *}\end{array}$ \\
\hline $\begin{array}{l}\text { Category x } \\
\text { Group }\end{array}$ & $\begin{array}{l}3.22 \\
(=0.08)\end{array}$ & $\begin{array}{l}7.98 \\
(=0.008)^{*}\end{array}$ & & & $\begin{array}{l}0.60 \\
(=0.45)\end{array}$ & $\begin{array}{l}2.95 \\
(=0.09)\end{array}$ \\
\hline $\begin{array}{l}\text { PD-nonVH, } \\
\text { PD-VH, } \\
\text { RT, Recognit } \\
\text { recognition ti } \\
\text { faces vs. } \\
\star * ~ p<0.005 \\
0.025) \text { \# }<\end{array}$ & $\begin{array}{l}\text { Parkinson } \\
\text { Parkinson } \\
\text { tion times; CVF } \\
\text { mes Between-s } \\
\text { ar (except fo } \\
\text { Significant be } \\
0.05 \text { Significant }\end{array}$ & $\begin{array}{l}\text { patients } \\
\text { patients } \\
\text { RT, Mean indivi } \\
\text { subject factor: G } \\
\text { or PES and } \\
\text { tween and with } \\
\text { effects without }\end{array}$ & $\begin{array}{l}\text { without visu } \\
\text { ith visual } \\
\text { idual variability } \\
\text { Group: PD-non } \\
\text { Proportion err } \\
\text { hin main and ir } \\
\text { Bonferroni corr }\end{array}$ & $\begin{array}{l}\text { hallucina } \\
\text { hallucinatio } \\
\text { coefficients (in } \\
\text { VH vs. PD-VH } \\
\text { oneous recog } \\
\text { teraction effec } \\
\text { ection. }\end{array}$ & $\begin{array}{l}\text { ations } \\
\text { ons } \\
\text { dividual SD/ir } \\
\text { Within-subjec } \\
\text { nition in Sc } \\
\text { ts (Bonferron }\end{array}$ & $\begin{array}{l}\quad=16) \\
=\quad 16 \\
\text { lividual mean) } \\
\text { factor: Category } \\
\text { ambled images } \\
\text { correction * } p\end{array}$ \\
\hline
\end{tabular}




\subsubsection{CFS condition: all perceptual measures}

To investigate how perceptual awareness might contribute to the sensitivity of behavioral markers of $\mathrm{VH}$, we also assessed perceptual errors, RT and CVRT in the 'invisible' condition created by using CFS (Tsuchiya \& Koch, 2005) (Supplementary Table S2.4). In the CFS condition, healthy controls and PD patients did not differ in the proportion correct recognition $(F(1,55)=2.81, p=0.10)$, proportion missed responses $(F(1,55)=0.93, p=0.34)$, proportion misperceived scrambled images $(F(1,55)=1.21, p$ $=0.28)$ and or PES $(F(1,55)=3.33, p=0.07)$ (ANOVA_1, Supplementary Table S2.5).

Comparable to the non-CFS condition, PD-VH patients made more image recognition errors $(F(1,30)=8.18, p=0.008)$, and missed more responses than the PD-nonVH $(F(1,30)=7.01, p=0.01)$ (ANOVA_2, Supplementary Table S2.6). These differences were not significant in the ANCOVA model with LEDD and disease duration as covariates $(p>0.07)$ (Supplementary Table S2.7). PD-VH had a higher perceptual error score (ANOVA_2, $F(1,30)=8.27, p=0.007 ;$ ANCOVA, $F(1,28)=7.66, p=0.01$ ) (ANOVA_2, Supplementary Table S2.6, ANCOVA, Supplementary Table S2.7). PD patients did not need more time to respond and did not differ in respect to variability of $\mathrm{RT}$ in comparison to $\mathrm{HC}(\mathrm{p}>0.57)$ (ANOVA_1, Supplementary Table S2.5). In contrast to the non-CFS condition here was no difference in RT and variability of RT between PD-nonVH and PD-VH ( $p>0.19)$ (ANOVA_2, Supplementary Table S2.6). Most likely, the lack of group RT effects in the CFS condition is due to the fact that recognition times in the CFS condition vary strongly across trials in all groups due to the additional perceptual suppression component. 


\subsubsection{Resting state}

We first tested group differences of network functional connectivity estimates of the eight networks of interest using univariate ANOVAs. This analysis showed no significant differences between $\mathrm{HC}$ and PD patients (default mode: $F(1,33)=1.94, p=0.17$, dorsal attention: $F(1,33)=2.15, p=0.15$, Salience: $F(1,33)=1.19, p=0.28$, left fronto-parietal: $F(1,33)=0.03, p=0.86$, right fronto-parietal: $F(1,33)=1.78, p=0.19$, somatomotor: $F(1,33)=3.89, p=0.06$, Visual medial: $F(1,33)=0.16, p=0.69$, Visual lateral: $F(1,33)=$ $0.02, p=0.9$ ). Given that we had a smaller subsample of PD patients, (10 PD-nonVH and 6 PD-VH,), we did not compare the different PD patient groups. Functional connectivity maps of $\mathrm{HC}$ and PD patients are presented in Figure 2.2.

\subsubsection{Correlation of Resting state functional connectivity and Perceptual Error score (PES), intra-individual variability of recognition times (CVRT)}

Spearman's rho correlations were used to investigate the relationship between PES, CVRT and resting state network functional connectivity in the PD group. To ensure reliability of our findings we also performed partial correlations including disease duration and LEDD as control variables. The partial correlations for all networks of interest are summarized in Supplementary Table S2.8.

Among the PD patients, perceptual error scores $(\log (P E S+1)$ were negatively correlated with functional connectivity between the left fronto-parietal network and the somatomotor network ( $r s=-0.56, p=0.03)$ ), which did not remain stable after performing partial correlations including LEDD and disease duration $(r=-0.12, p=$ 0.72). PES was also negatively correlated with functional connectivity between the 
dorsal attention network and salience network $(r s=-0.50, p=0.049)$ which remained stable after performing partial correlations including LEDD and disease duration $(r=-$ 0.54, $p=0.047$ ). (Figure 2.7A, see Supplementary Table S2.8, for values of all networks). Among the PD patients, CVRT was negatively correlated to functional connectivity between the right fronto-parietal network and default mode network ( $\mathrm{rs}=$ $0.54, p=0.03$ ) which did not remain stable including LEDD and disease duration as control variables $(r=-0.53, p=0.053)$. CVRT was also negatively correlated with functional connectivity between somatomotor network and right fronto-parietal network ( $r s=-0.51, p=0.044)$ which remained stable after performing partial correlations using LEDD and disease duration as control variables $(r=-0.55, p=0.041)$ (Figure 2.7B,

\section{Supplementary Table S2.8).}

A

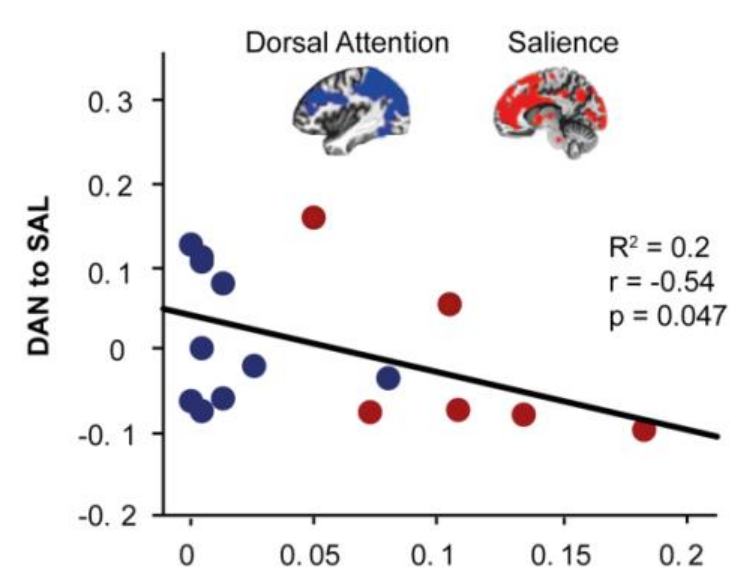

$\log ($ Perceptual Error Score+1)

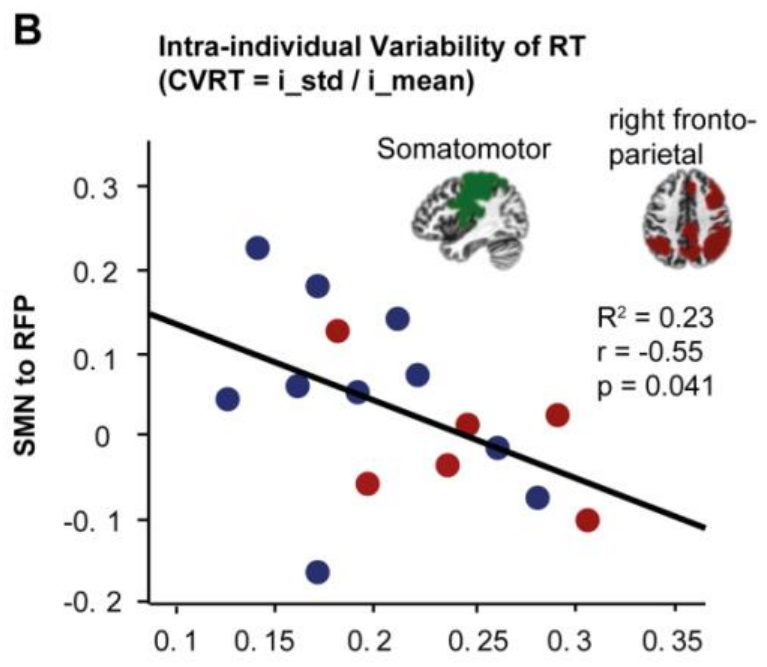

Coefficient of RT variability(CVRT)

\section{PD-VH PD-nonVH}

Figure 2.7. Within- and between-network connectivity associated with perceptual error score (PES) and individual variability of RT. (A) Scatter plot of logarithmic transformation of the PES on the $x$ axis and connectivity of the dorsal attention network to salience networks on the y axis of PD patients ( $N$ 
= 16). (B) Scatter plot of CVRT on the $x$ axis and connectivity of somatomotor (SMN) to right frontoparietal networks on the $y$ axis $(N=16)$. Blue dots show the individual values of PD-nonVH $(N=10)$ and red dots of PD-VH $(\mathrm{N}=6)$. 


\subsection{Discussion}

We showed that perceptual errors and elevated intra-individual behavioral variability characterize PD patients with self-reported hallucinations compared to PD patients not reporting $\mathrm{VH}$. We showed that these behavioral measures are associated with functional connectivity changes in overlapping, but slightly different neural networks: higher PES correlated with lower functional connectivity between dorsal attention network and salience network, while higher intra-subject variability in RT correlated with lower functional connectivity between somatomotor and right fronto-parietal networks.

\subsubsection{Comparison with previous findings on misperceptions in PD-VH patients}

In accordance with previous studies, PD-VH patients exhibited lower rates of correct image recognition and reported real images in scrambled images more often than PDnonVH (Meppelink et al., 2008; Ramirez-Ruiz et al., 2007; Shine et al., 2012). It remains to be investigated which score is more sensitive for the identification of hallucinating patients and which can serve best to identify patients experiencing or being at risk to develop hallucinations. Consistent with previous research we found a negative correlation between misperceptions and functional connectivity between dorsal attention network and the salience network, (Shine, Keogh, et al., 2015). The salience network is related to the degree of personal salience in the cognitive, emotional or homeostatic domain and interoceptive-autonomic processing (Seeley et al., 2007). Distortion in attentional processes might be a common mechanism for the development of visual hallucinations given that attentional deficits have also been shown in hallucinating patients suffering from Charles Bonnet syndrome and dementia (Graham et al., 2011; Makin et al., 2013). 


\subsubsection{Intra-individual variability in recognition times}

PD-VH patients showed higher intra-individual variability in recognition times than PDnonVH, which to our knowledge has not been reported before. Performance fluctuations are problematic for the development of objective measures for $\mathrm{VH}$ based on mean performance (MacDonald et al., 2009). At the same time, intra-individual variability appears to be a promising variable as previous research indicates that trial-by-trial fluctuations in RT correlate negatively with cognitive performance and can even serve as a sensitive predictor for age-related cognitive decline (Bielak, Hultsch, Strauss, MacDonald, \& Hunter, 2010; MacDonald, Nyberg, \& Bäckman, 2006), incipient dementia in PD (de Frias et al., 2012) and identification of individuals at risk to develop hallucinations such as persons at risk for schizophrenia (Shin et al., 2013). In healthy aging populations, larger intra-individual variability has been linked to attentional lapses (Bunce, Warr, \& Cochrane, 1993) and deficits in executive control (West, Murphy, Armilio, Craik, \& Stuss, 2002). Since previous studies reported a strong link between visual hallucinations in PD and deficits in attentional and executive processes (Barnes, Boubert, Harris, Lee, \& David, 2003; Gallagher et al., 2011; Hall et al., 2016; Meppelink et al., 2008), this functional interpretation is plausible (Muller et al., 2014; Shine et al., 2014). Concerning neural correlates of performance variability, the most consistent correlation with increased intra-individual variability in healthy elderly and other patient groups have been found with structural and functional alterations of prefrontal cortices (MacDonald et al., 2006; Murtha, Cismaru, Waechter, \& Chertkow, 2002) and alterations in DMN and attentional networks (Kelly, Uddin, Biswal, Castellanos, \& Milham, 2008), which both include prefrontal regions. We found that PD patients with 
higher intra-individual variability show reduced functional connectivity between somatomotor and right fronto-parietal networks. Previous research reported hyperconnectivity of fronto-parietal regions in hallucinating PD patients compared to non-hallucinating PD patients (Franciotti et al., 2015; Yao et al., 2014). The sensorimotor network is important for voluntary movements and shows deviant functional connectivity in PD (Biswal, Yetkin, Haughton, \& Hyde, 1995). However, exploratory analyses of our data show that the severity of the motor symptoms measured with the UPDRS score III was not related to functional connectivity between SMN and right fronto-parietal network using LEDD and disease duration as covariates, indicating that the correlation between CVRT and functional connectivity between SMN and right fronto-parietal network is not driven by movement disabilities in PD $(r=0.17, p$ $=0.62$. Hypoconnectivity within the somatosensory and between fronto-parietal and ventral attentional network has also been reported in schizophrenia which indicates their possible involvement in hallucinations (Dong, Wang, Chang, Luo, \& Yao, 2018). Furthermore, during working memory maintenance patients with mild cognitive impairment show hypoactivation of right fronto-parietal regions (Melrose et al., 2018) indicating its influence in cognitive decline. Increased intra-individual behavioral fluctuations were reported in multiple other diseases such as schizophrenia, autism, attention deficit hyperactivity disorder (ADHD) as well as in aging populations exhibiting cognitive decline (Dinstein, Heeger, \& Behrmann, 2015; MacDonald, Li, \& Bäckman, 2009). Thus, the intra-individual variability observed in our task together with the hypoconnectivity of the somatomotor and fronto-parietal networks might be useful to predict cognitive changes in PD patients rather than the occurrence of visual 
hallucinations itself.

\subsubsection{Limitations}

One limitation is that we cannot be sure about the type of misperceptions participants experienced during the presentation of scrambled images. As such there is a tradeoff between verbally reporting patients providing more insight into phenomenology, and trial-based measures offering the opportunity to collect many trials of the same type. Another limitation of the current study is the relatively small sample size of the demographically matched PD patients and the small subsample participating in the resting state. For this reason the correlational analyses of resting state functional connectivity need to be interpreted with caution as the subsample did not provide sufficient power to apply Bonferroni correction with confidence about possible negative results. Clearly, a study with a larger PD sample where it is not only possible to control, but to match the patients for clinical variables such as LEDD and disease duration, is warranted. Albeit we tested for visual impairments and general cognitive state, we did not include assessments of attentional or executive functions in our study. Future studies would also profit from the identification of different phenotypes in cognition and attention such as memory, sustained, selective, alternating and divided attention. Moreover, differences in phenotype profiles of different clinical groups such as patients with mild cognitive impairments and different types of dementia would also be helpful to distill the best predictors for hallucinations versus cognitive decline. 


\section{References}

Armstrong, R. A. (2011). Visual Symptoms in Parkinson's Disease. Parkinson's Disease, 2011. https://doi.org/10.4061/2011/908306

Baggio, H. C., Segura, B., Sala-Llonch, R., Marti, M. J., Valldeoriola, F., Compta, Y., ... Junqué, C. (2015). Cognitive impairment and resting-state network connectivity in Parkinson's disease. Human Brain Mapping, 36(1), 199-212. https://doi.org/10.1002/hbm.22622

Bartlett, M. S. (1947). The Use of Transformations. Biometrics, 3(1), 39-52. https://doi.org/10.2307/3001536

Beck, A. T., Ward, C. H., Mendelson, M., Mock, J., \& Erbaugh, J. (1961). An inventory for measuring depression. Archives of General Psychiatry, 4, 561-571.

Beckmann, C. F., Mackay, C. E., Filippini, N., \& Smith, S. M. (2009). Group comparison of resting-state FMRI data using multi-subject ICA and dual regression. Neuroimage, 47(Suppl 1), S148.

Behzadi, Y., Restom, K., Liau, J., \& Liu, T. T. (2007). A component based noise correction method (CompCor) for BOLD and perfusion based fMRI. Neurolmage, 37(1), 90-101. https://doi.org/10.1016/j.neuroimage.2007.04.042

Bielak, A. A., Hultsch, D. F., Strauss, E., MacDonald, S. W., \& Hunter, M. A. (2010). Intraindividual variability in reaction time predicts cognitive outcomes 5 years later. Neuropsychology, 24(6), 731.

Camicioli, R. M., Wieler, M., de Frias, C. M., \& Martin, W. R. W. (2008). Early, untreated Parkinson's disease patients show reaction time variability. Neuroscience Letters, 441(1), 77-80. https://doi.org/10.1016/j.neulet.2008.06.004 
Collerton, D., Perry, E., \& McKeith, I. (2005). Why people see things that are not there: a novel Perception and Attention Deficit model for recurrent complex visual hallucinations. The Behavioral and Brain Sciences, 28(6), 737-757; discussion 757-794. https://doi.org/10.1017/S0140525X05000130

de Frias, C. M., Dixon, R. A., \& Camicioli, R. (2012). Neurocognitive speed and inconsistency in Parkinson's disease with and without incipient dementia: an 18month prospective cohort study. Journal of the International Neuropsychological Society: JINS, 18(4), 764-772. https://doi.org/10.1017/S1355617712000422 de Frias, C. M., Dixon, R. A., Fisher, N., \& Camicioli, R. (2007). Intraindividual variability in neurocognitive speed: a comparison of Parkinson's disease and normal older adults. Neuropsychologia, 45(11), 2499-2507. https://doi.org/10.1016/j.neuropsychologia.2007.03.022

Diederich, N. J., Fénelon, G., Stebbins, G., \& Goetz, C. G. (2009). Hallucinations in Parkinson disease. Nature Reviews. Neurology, 5(6), 331-342. https://doi.org/10.1038/nrneurol.2009.62

Ekman, P., \& Friesen, W. V. (1976). Pictures of Facial Affect. Palo Alto.CA.Colsulting Psychologists Press.

Fassbender, C., Lesh, T. A., Ursu, S., \& Salo, R. (2015). Reaction Time Variability and Related Brain Activity in Methamphetamine Psychosis. Biological Psychiatry, 77(5), 465-474. https://doi.org/10.1016/j.biopsych.2014.07.028

Fassbender, C., Scangos, K., Lesh, T. A., \& Carter, C. S. (2014). RT distributional analysis of cognitive-control-related brain activity in first-episode schizophrenia. 
Cognitive, Affective \& Behavioral Neuroscience, 14(1), 175-188.

https://doi.org/10.3758/s13415-014-0252-4

Flehmig, H. C., Steinborn, M., Langner, R., Scholz, A., \& Westhoff, K. (2007). Assessing intraindividual variability in sustained attention: reliability, relation to speed and accuracy, and practice effects. Psychology Science, 49(2), 132-149.

Folstein, M. F., Folstein, S. E., \& McHugh, P. R. (1975). "Mini-mental state". A practical method for grading the cognitive state of patients for the clinician. Journal of Psychiatric Research, 12(3), 189-198.

Goetz, C.G., Tilley, B.C., Shaftman, S.R., Stebbins, G.T., Fahn, S., Martinez-Martin, P., Poewe, W., Sampaio, C., Stern, M.B., Dodel, R., Dubois, B., Holloway, R., Jankovic, J., Kulisevsky, J., Lang, A.E., Lees, A., Leurgans, S., LeWitt, P.A., Nyenhuis, D., Olanow, C.W., Rascol, O., Schrag, A., Teresi, J.A., Hilten, J.J. van, LaPelle, N., 2008. Movement Disorder Society-sponsored revision of the Unified Parkinson's Disease Rating Scale (MDS-UPDRS): Scale presentation and clinimetric testing results. Movement Disorders 23, 2129-2170. https://doi.org/10.1002/mds.22340

Graham, G., Dean, J., Mosimann, U. P., Colbourn, C., Dudley, R., Clarke, M., \& Collerton, D. (2011). Specific attentional impairments and complex visual hallucinations in eye disease. International Journal of Geriatric Psychiatry, 26(3), 263-267. https://doi.org/10.1002/gps.2522

Gupta, M., Singh, G., Khwaja, G. A., \& Mehndiratta, M. M. (2004). Hallucinations in Parkinson's disease--a study of forty three patients. The Journal of the Association of Physicians of India, 52, 703-706. 
Koerts, J., Borg, M. A. J. P., Meppelink, A. M., Leenders, K. L., van Beilen, M., \& van Laar, T. (2010). Attentional and perceptual impairments in Parkinson's disease with visual hallucinations. Parkinsonism \& Related Disorders, 16(4), 270-274. https://doi.org/10.1016/j.parkreldis.2010.01.003

Kritzinger, C., Vollstedt, E.-J., Hückelheim, K., Lorwin, A., Graf, J., Tunc, S., ... Kasten, M. (2015). Qualitative Characteristics of Depression in Parkinson's Patients and Controls. Behavioural Neurology, 2015, 961372.

https://doi.org/10.1155/2015/961372

MacDonald, S. W. S., Li, S.-C., \& Bäckman, L. (2009). Neural underpinnings of withinperson variability in cognitive functioning. Psychology and Aging, 24(4), 792-808. https://doi.org/10.1037/a0017798

MacDonald, S. W. S., Nyberg, L., \& Bäckman, L. (2006). Intra-individual variability in behavior: links to brain structure, neurotransmission and neuronal activity. Trends in Neurosciences, 29(8), 474-480. https://doi.org/10.1016/j.tins.2006.06.011

Makin, S. M., Redman, J., Mosimann, U. P., Dudley, R., Clarke, M. P., Colbourn, C., \& Collerton, D. (2013). Complex visual hallucinations and attentional performance in eye disease and dementia: a test of the Perception and Attention Deficit model. International Journal of Geriatric Psychiatry, 28(12), 1232-1238. https://doi.org/10.1002/gps.3947

Manni, R., Terzaghi, M., Ratti, P.-L., Repetto, A., Zangaglia, R., \& Pacchetti, C. (2011). Hallucinations and REM sleep behaviour disorder in Parkinson's disease: dream 
imagery intrusions and other hypotheses. Consciousness and Cognition, 20(4), 1021-1026. https://doi.org/10.1016/j.concog.2010.10.009

Maria, B., Sophia, S., Michalis, M., Charalampos, L., Andreas, P., John, M. E., \& Nikolaos, S. M. (2003). Sleep breathing disorders in patients with idiopathic Parkinson's disease. Respiratory Medicine, 97(10), 1151-1157.

Matsui, H., Udaka, F., Tamura, A., Oda, M., Kubori, T., Nishinaka, K., \& Kameyama, M. (2006). Impaired visual acuity as a risk factor for visual hallucinations in Parkinson's disease. Journal of Geriatric Psychiatry and Neurology, 19(1), 3640. https://doi.org/10.1177/0891988705284739

Meppelink, A. M., de Jong, B. M., Renken, R., Leenders, K. L., Cornelissen, F. W., \& van Laar, T. (2009). Impaired visual processing preceding image recognition in Parkinson's disease patients with visual hallucinations. Brain: A Journal of Neurology, 132(Pt 11), 2980-2993. https://doi.org/10.1093/brain/awp223

Meppelink, A. M., Koerts, J., Borg, M., Leenders, K. L., \& van Laar, T. (2008). Visual object recognition and attention in Parkinson's disease patients with visual hallucinations. Movement Disorders: Official Journal of the Movement Disorder Society, 23(13), 1906-1912. https://doi.org/10.1002/mds.22270

Moeller, S., Yacoub, E., Olman, C. A., Auerbach, E., Strupp, J., Harel, N., \& Uğurbil, K. (2010). Multiband multislice GE-EPI at 7 tesla, with 16-fold acceleration using partial parallel imaging with application to high spatial and temporal whole-brain fMRI. Magnetic Resonance in Medicine, 63(5), 1144-1153. https://doi.org/10.1002/mrm.22361 
Morrison, A. P., Wells, A., \& Nothard, S. (2000). Cognitive factors in predisposition to auditory and visual hallucinations. The British Journal of Clinical Psychology, 39 ( Pt 1), 67-78.

Muller, A. J., Shine, J. M., Halliday, G. M., \& Lewis, S. J. G. (2014). Visual hallucinations in Parkinson's disease: theoretical models. Movement Disorders: Official Journal of the Movement Disorder Society, 29(13), 1591-1598. https://doi.org/10.1002/mds.26004

Onofrj, M., Taylor, J. P., Monaco, D., Franciotti, R., Anzellotti, F., Bonanni, L., ... Thomas, A. (2013). Visual hallucinations in PD and Lewy body dementias: old and new hypotheses. Behavioural Neurology, 27(4), 479-493. https://doi.org/10.3233/BEN-129022

Papapetropoulos, S., Katzen, H., Schrag, A., Singer, C., Scanlon, B. K., Nation, D., ... Levin, B. (2008). A questionnaire-based (UM-PDHQ) study of hallucinations in Parkinson's disease. BMC Neurology, 8, 21. https://doi.org/10.1186/1471-2377$8-21$

Peraza, L. R., Kaiser, M., Firbank, M., Graziadio, S., Bonanni, L., Onofrj, M., ... Taylor, J.-P. (2014). fMRI resting state networks and their association with cognitive fluctuations in dementia with Lewy bodies. Neurolmage. Clinical, 4, 558-565. https://doi.org/10.1016/j.nicl.2014.03.013

Pieri, V., Diederich, N. J., Raman, R., \& Goetz, C. G. (2000). Decreased color discrimination and contrast sensitivity in Parkinson's disease. Journal of the Neurological Sciences, 172(1), 7-11. 
Prell, T. (2018). Structural and Functional Brain Patterns of Non-Motor Syndromes in Parkinson's Disease. Frontiers in Neurology, 9, 138. https://doi.org/10.3389/fneur.2018.00138

Ramirez-Ruiz, B., Junque, C., Marti, M.-J., Valldeoriola, F., \& Tolosa, E. (2007).

Cognitive changes in Parkinson's disease patients with visual hallucinations. Dementia and Geriatric Cognitive Disorders, 23(5), 281-288. https://doi.org/10.1159/000100850

Rentrop, M., Rodewald, K., Roth, A., Simon, J., Walther, S., Fiedler, P., ... Kaiser, S. (2010). Intra-individual variability in high-functioning patients with schizophrenia. Psychiatry Research, 178(1), 27-32. https://doi.org/10.1016/j.psychres.2010.04.009

Seeley, W. W., Menon, V., Schatzberg, A. F., Keller, J., Glover, G. H., Kenna, H., ... Greicius, M. D. (2007). Dissociable intrinsic connectivity networks for salience processing and executive control. The Journal of Neuroscience: The Official Journal of the Society for Neuroscience, 27(9), 2349-2356. https://doi.org/10.1523/JNEUROSCI.5587-06.2007

Setsompop, K., Gagoski, B. A., Polimeni, J. R., Witzel, T., Wedeen, V. J., \& Wald, L. L. (2012). Blipped-controlled aliasing in parallel imaging for simultaneous multislice echo planar imaging with reduced g-factor penalty. Magnetic Resonance in Medicine, 67(5), 1210-1224. https://doi.org/10.1002/mrm.23097

Shin, Y. S., Kim, S. N., Shin, N. Y., Jung, W. H., Hur, J.-W., Byun, M. S., ... Kwon, J. S. (2013). Increased intra-individual variability of cognitive processing in subjects at 
risk mental state and schizophrenia patients. PloS One, 8(11), e78354. https://doi.org/10.1371/journal.pone.0078354

Shine, J. M., Halliday, G. H., Carlos, M., Naismith, S. L., \& Lewis, S. J. G. (2012). Investigating visual misperceptions in Parkinson's disease: a novel behavioral paradigm. Movement Disorders: Official Journal of the Movement Disorder Society, 27(4), 500-505. https://doi.org/10.1002/mds.24900

Shine, J. M., Halliday, G. M., Gilat, M., Matar, E., Bolitho, S. J., Carlos, M., ... Lewis, S. J. G. (2014). The role of dysfunctional attentional control networks in visual misperceptions in Parkinson's disease. Human Brain Mapping, 35(5), 22062219. https://doi.org/10.1002/hbm.22321

Shine, J. M., Keogh, R., O'Callaghan, C., Muller, A. J., Lewis, S. J. G., \& Pearson, J. (2015). Imagine that: elevated sensory strength of mental imagery in individuals with Parkinson's disease and visual hallucinations. Proceedings. Biological Sciences / The Royal Society, 282(1798), 20142047. https://doi.org/10.1098/rspb.2014.2047

Shine, J. M., Muller, A. J., O'Callaghan, C., Hornberger, M., Halliday, G. M., \& Lewis, S. J. (2015). Abnormal connectivity between the default mode and the visual system underlies the manifestation of visual hallucinations in Parkinson's disease: a task-based fMRI study. NPJ Parkinson's Disease, 1, 15003.

https://doi.org/10.1038/npjparkd.2015.3

Tomlinson, C. L., Stowe, R., Patel, S., Rick, C., Gray, R., \& Clarke, C. E. (2010). Systematic review of levodopa dose equivalency reporting in Parkinson's 
disease. Movement Disorders: Official Journal of the Movement Disorder

Society, 25(15), 2649-2653. https://doi.org/10.1002/mds.23429

Tsuchiya, N., \& Koch, C. (2005). Continuous flash suppression reduces negative afterimages. Nature Neuroscience, 8(8), 1096-1101. https://doi.org/10.1038/nn1500

Wilke, M. (2012). An iterative jackknife approach for assessing reliability and power of FMRI group analyses. PloS One, 7(4), e35578. https://doi.org/10.1371/journal.pone.0035578

\section{Ethics}

The study was approved by the medical ethics committee of the University Medical Center Goettingen, Germany. All participants signed an informed consent form prior to the examination.

\section{Acknowledgements}

We thank Severin Heumüller for excellent computer and programming support and Dr. Holger Sennhenn-Reulen for helpful statistical discussions.

\section{Author Contributions}

(1) Research Project: A. Conception, B. Organization, C. Execution, Supervision; (2) Statistical Analysis: A. Design, B. Execution, C. Review and Critique; (3) Manuscript Preparation: A. Writing of the First Draft, B. Review and Critique. 
K.M.: 1A, 1B, 1C, 2A, 2B, 3A, 3B; C.S.: 1A, 2A, 2B, 2C, 3B; K.W.: 2A, 2B, 2C, 3B;

C.W.: 1B, 1C, 3B; C.T.: 1B, 1C, 3B; K.B.: 1B, 1C, 3B; M.B.: 1B, 1C, 3B; M.W.: 1A, 1C, $2 \mathrm{~A}, 2 \mathrm{~B}, 2 \mathrm{C}, 3 \mathrm{~A}, 3 \mathrm{~B}$.

\section{Funding}

This work was supported by the Herman and Lilly Schilling Foundation (MW), the DFG Center for Nanoscale Microscopy \& Molecular Physiology of the Brain (CNMPB) and the Leibniz-Science Campus Primate Cognition. 


\section{Supplementary Materials}

Misperceptions and intra-individual variability in Parkinson's disease:

a behavioral and fMRI study

Kristina Miloserdov et al.

Supplementary Materials contains:

Supplementary Methods and Materials (Apparatus and Stimuli)

2 Supplementary Figures

8 Supplementary Tables 


\section{Supplementary Methods and Materials}

\section{S.2.1.1. Apparatus and Stimuli}

Stimuli were presented on a 27 inch LCD monitor. The screen resolution was $1920 \mathrm{x}$ 1080 pixels. The monitor had a vertical refresh rate of $60 \mathrm{~Hz}$. Participants were sitting in a dimly lit room. The eye-to-screen distance was $57 \mathrm{~cm}$. Visual stimuli were presented with custom-written software using Matlab (Version R2011b, 32bit) and the Psychtoolbox for Microsoft Windows (Brainard, 1997). Responses were recorded with a USB button-box and behavioral responses were recorded in Matlab and stored together with the stimulus information on an x64-based PC. Target stimuli consisted of photographs of cars (http://vision.caltech.edu/archive.html), faces (Ekman \& Friesen, 1976) or their scrambled versions, presented in a pseudorandom manner. Each image subtended 480 pixels by 480 pixels and was presented in the center of the screen. Face stimuli were randomly drawn from different facial expressions. Scrambled images were generated using Matlab by dividing the images in $10 \times 10$ pixels blocks and randomizing their position (Figure 2.1A). Target images were presented monocular to the right eye by using red-blue goggles glasses and displaying the image solely by the blue gun (stereomode of the psychophysics toolbox (Carmel, Arcaro, Kastner, \& Hasson, 2010)). In CFS trials, which were designed to suppress the images from visual awareness, high contrast and rapidly changing red-colored Mondrian patterns were flashed to the left eye at a frequency of $10 \mathrm{~Hz}$ (Tsuchiya \& Koch, 2005). Thus, the right eye perceived the target image and the left eye perceived the Mondrian mask (Carmel et al., 2010) (Figure 2.1C). Mondrian patterns were drawn from a pool of 100 randomly generated patterns (http://martin-hebart.de/webpages/code/stimuli.html) (Stein, Hebart, \& Sterzer, 
2011). In the non-CFS condition, only the target image was presented to the right eye without the mask (Figure 2.1B).

\section{Supplementary Figures}

A Perceptual Error Score

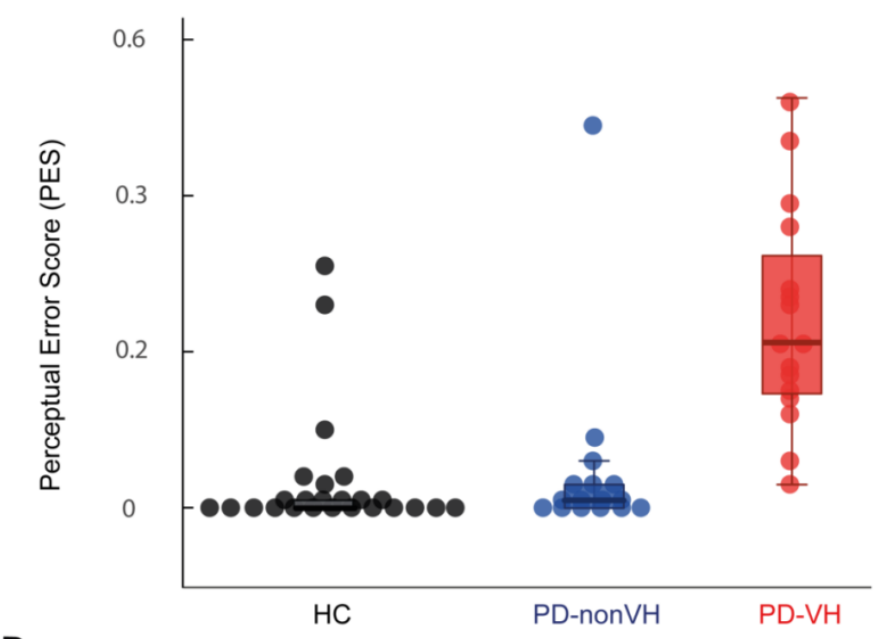

B

Individual Variability of RT (CVRT = i_std / i_mean)

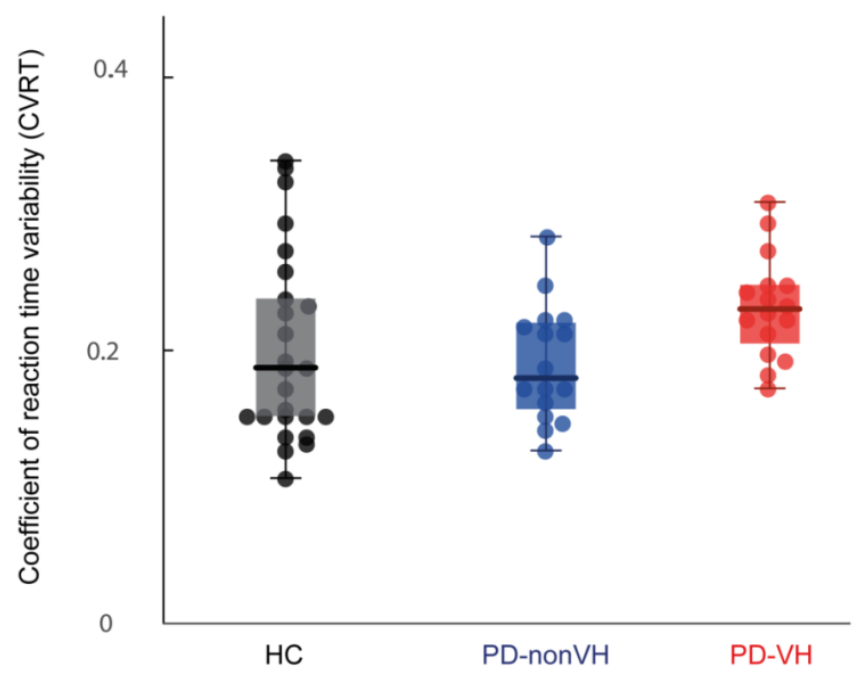

Supplementary Figure S2.1. Individual Perceptual Error and Intra-Individual Variability scores. (A) Individual PES values and boxplots illustrating the median in the center of the box, error bars the 95\% confidence interval separated for $\mathrm{HC}(\mathrm{N}=25)$ in black, PD-nonVH $(\mathrm{N}=16)$ in blue and PD-VH $(\mathrm{N}=16)$ in red. (B) Individual CVRT and boxplots illustrating the median in the center of the box, error bars the $95 \%$ confidence interval separated values separated for $\mathrm{HC}(\mathrm{N}=25)$ in black, PD-nonVH $(\mathrm{N}=16)$ in blue and PD-VH $(\mathrm{N}=16)$ in red. 


\section{A Recognition times across trials}

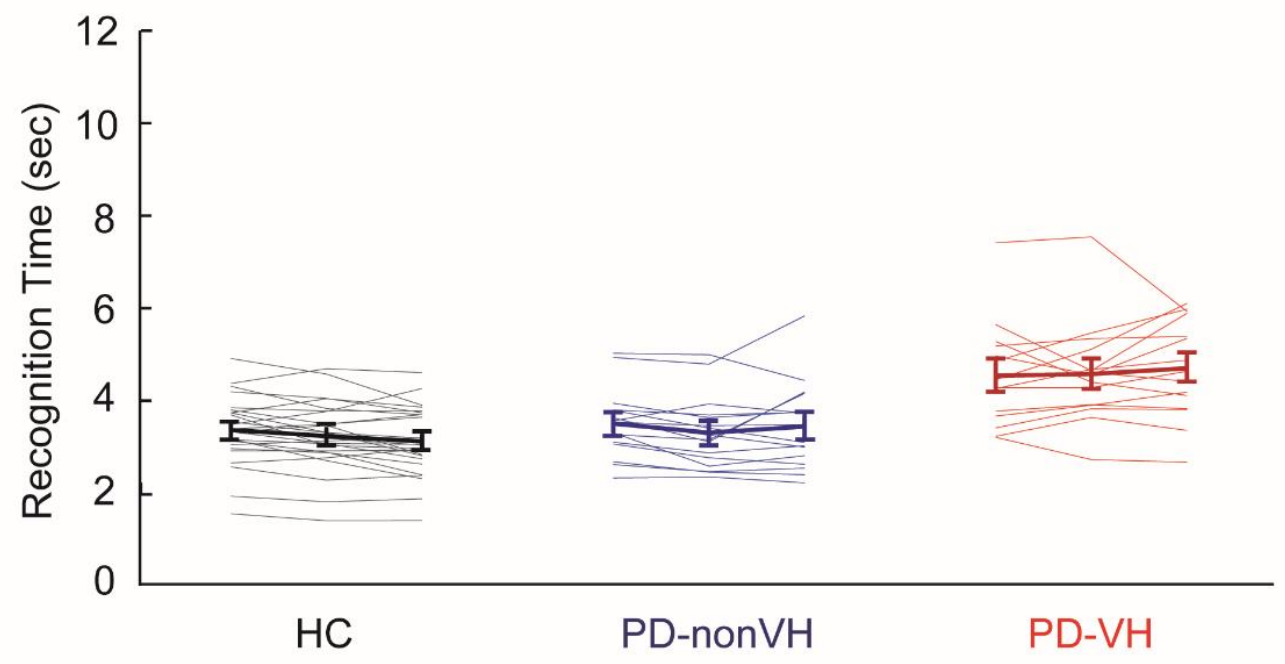

B

\section{Recognition times across trials}

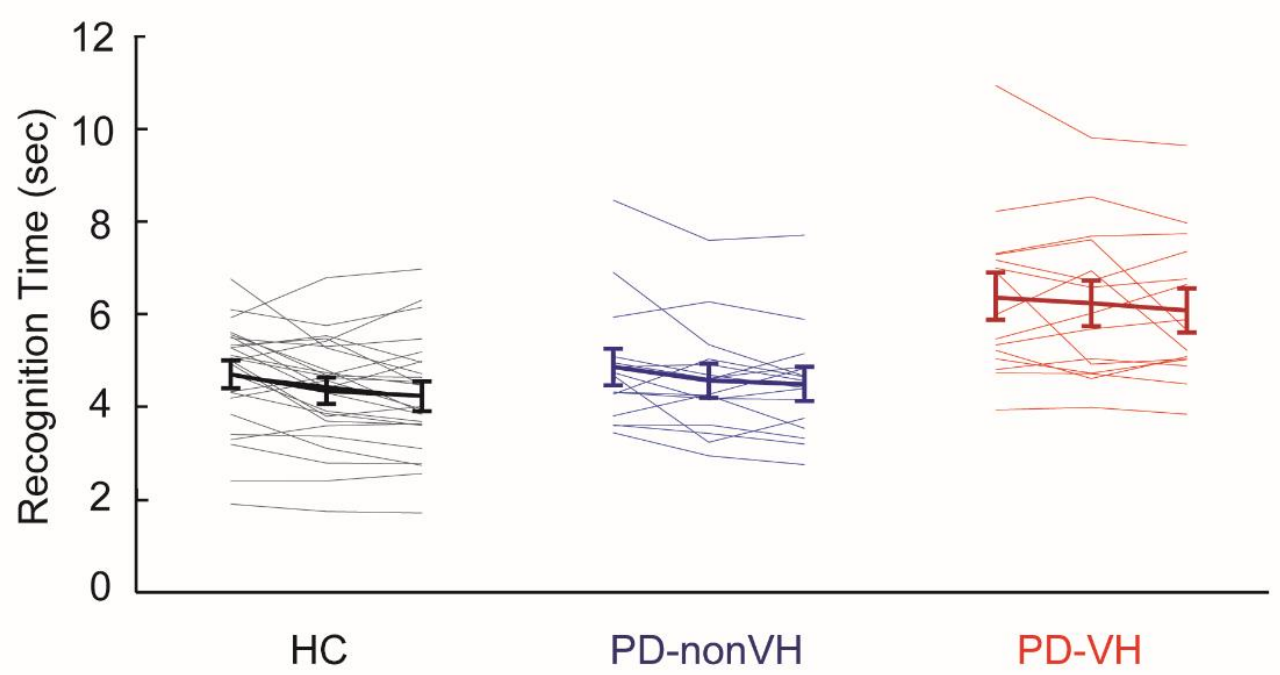

Supplementary Figure S2.2. Recognition time across trials. (A) Lines show mean and individual recognition times for the three intervals for face images of the non-CFS condition. Each bin corresponds to one third of correct trials. (B) Lines show mean and individual recognition times for the three time intervals, each interval consisting of one third of correct trials, of the non-CFS condition for car images. In (A) and (B), error bars show means and S.E.M. across subjects. HC: Healthy controls, PD-nonVH: Parkinson patients without visual hallucinations and PD-VH: Parkinson patients with visual hallucinations 


\section{Supplementary Tables}

Table S2.1. Demographic and neuropsychological characteristics of subjects that participated in the Resting state fMRI and image recognition task

\begin{tabular}{ccc}
\hline & $\begin{array}{c}\mathrm{HC} \\
(\mathrm{N}=19)\end{array}$ & $\begin{array}{c}\mathrm{PD} \\
(\mathrm{N}=16)\end{array}$ \\
\cline { 2 - 3 } & Mean (SD) & Mean (SD) \\
\hline Age (years) & $68.32(6.30)$ & $69.44(8.52)$ \\
\hline Gender (female/male), $\mathrm{n}(\%)$ & $0(0 \%) /$ & $3(19 \%) /$ \\
& $19(100 \%)$ & $13(81 \%)$ \\
\hline Education years & $3.74(2.06)$ & $3.44(1.62)$ \\
\hline MMSE & $29.16(0.90)$ & $28.38(1.31)$ \\
\hline Hoehn and Yahr stage & - & $2.06(0.70)$ \\
\hline Disease duration & - & $20.67(6.99)$ \\
\hline UPDRS III & - & $614.78(397.31)$ \\
\hline LEDD, mg/day & - & $3.63(4.89)$ \\
\hline UM-PDHQ & $6.81(4.64)$ \\
\hline BDI & $3.32(2.83)$ & $92.50(10.00)$ \\
\hline Visual acuity (\%) & $97.89(6.31)$ & $1.72(0.08)$ \\
\hline Mars Letters Contrast Sensitivity Test & $1.73(0.05)$ &
\end{tabular}

BDI, Beck's, Depression Inventory; HC, healthy controls; LEDD, levodopa-equivalent daily dose;

MMSE, Mini Mental State Examination; PD, Parkinson patients

UM-PDHQ, University of Miami Parkinson's disease Hallucinations Questionnaire

UPDRS, Unified Parkinson's Disease Rating Scale; 
Supplementary Table S2.2. Results from ANOVA_1 in the non-CFS condition: Repeated Measures mixed ANOVA with the factors Category, Group and their interaction effects for Healthy controls (HC) Parkinson patients (PD) in the non-CFS condition

\begin{tabular}{|c|c|c|c|c|c|c|}
\hline \multirow[t]{2}{*}{ Factor } & $\begin{array}{l}\text { Proportion } \\
\text { Correct } \\
\text { Recognition }\end{array}$ & $\begin{array}{l}\text { Proportion } \\
\text { Misses }\end{array}$ & $\begin{array}{l}\text { Proportion } \\
\text { Erroneous } \\
\text { Object } \\
\text { Recogni } \\
\text { tion in } \\
\text { Scrambled } \\
\text { Images }\end{array}$ & $\begin{array}{c}\text { Perceptual } \\
\text { Error } \\
\text { Score }\end{array}$ & CVRT & RT \\
\hline & $F(p)$ & $F(p)$ & $F(p)$ & $F(p)$ & $F(p)$ & $F(p)$ \\
\hline \multirow[t]{3}{*}{ Category } & 2.03 & 8.73 & & & 0.59 & 292.95 \\
\hline & $(=0.16)$ & $(=0.0046)^{\star \star}$ & & & $(=0.45)$ & $1<$ \\
\hline & & & & & & $0.00001)^{\star \star}$ \\
\hline \multirow[t]{2}{*}{ Group } & 9.52 & 3.16 & 4.86 & 9.81 & 0.47 & 8.87 \\
\hline & $(=0.003)^{\star \star}$ & $(=0.08)$ & $(=0.03) \#$ & $(=0.003)^{\star \star}$ & $(=0.50)$ & $(=0.004)^{\star \star}$ \\
\hline \multirow[t]{2}{*}{ Category $\mathrm{x}$} & 0.63 & 3.58 & & & 0.38 & 2.15 \\
\hline & $(=0.43)$ & $(=0.06)$ & & & $(=0.54)$ & $(=0.15)$ \\
\hline \multicolumn{7}{|c|}{$\begin{array}{l}\text { HC, Healthy controls }(\mathrm{N}=25) \text {; PD, Parkinson patients with and without visual hallucinations ( } \\
=32 \text { ) RT, Recognition times; CVRT, Mean individual variability coefficients (individual } \\
\text { SD/individual mean) of recognition times Between-subjects factor: Group: } 2 \text { Levels: HC, PD } \\
\text { Within-subjects factors: Category: } 2 \text { levels: face, car }{ }^{* *} p<0.005 \text { Significant between and } \\
\text { within main and interaction effects (Bonferroni correction } p<0.0025) \# p<0.05 \text { Significant } \\
\text { effects without Bonferroni correction }\end{array}$} \\
\hline
\end{tabular}


Supplementary Table S2.3. Results from the Repeated Measures mixed ANCOVA in the non-CFS condition: Repeated Measures mixed ANCOVA with the factors Category, Group, their interaction effects and the covariates disease duration and LEDD for Parkinson patients without visual hallucinations (PD-nonVH) and Parkinson patients with visual hallucinations (PD-VH)

\begin{tabular}{|c|c|c|c|c|c|c|}
\hline Factor & $\begin{array}{c}\text { Proportio } \\
\text { n Correct } \\
\text { Recogni } \\
\text { tion }\end{array}$ & $\begin{array}{c}\text { Proportion } \\
\text { Misses }\end{array}$ & $\begin{array}{l}\text { Proportion } \\
\text { Erroneous } \\
\text { Object } \\
\text { Recogni } \\
\text { tion in } \\
\text { Scrambled } \\
\text { Images }\end{array}$ & $\begin{array}{l}\text { Perceptual } \\
\text { Error Score }\end{array}$ & CVRT & RT \\
\hline & $F(p)$ & $F(p)$ & $F(p)$ & $F(p)$ & $F(p)$ & $F(p)$ \\
\hline \multirow[t]{3}{*}{ Category } & 1.78 & 1.03 & & & 1.21 & 22.78 \\
\hline & $(=0.19)$ & $(=0.32)$ & & & $(=0.28)$ & $(=$ \\
\hline & & & & & & $0.00005)^{\star *}$ \\
\hline \multirow[t]{3}{*}{ Group } & 1.96 & 0.94 & 12.75 & 13.88 & 4.34 & 2.78 \\
\hline & $(=0.17)$ & $(=0.34)$ & $(=0.001)^{\star *}$ & $(=0.001)^{\star *}$ & $(=$ & $(=0.11)$ \\
\hline & & & & & $0.047)^{*}$ & \\
\hline \multirow{2}{*}{$\begin{array}{l}\text { Disease } \\
\text { duration }\end{array}$} & 0.51 & 0.16 & 0.18 & 0.01 & 0.08 & 6.61 \\
\hline & $(=0.48)$ & $(=0.70)$ & $(=0.68)$ & $(=0.92)$ & $(=0.78)$ & $(=0.02)^{*}$ \\
\hline \multirow[t]{2}{*}{ LEDD } & 2.73 & 0.84 & 2.10 & 0.32 & 0.19 & 1.06 \\
\hline & $(=0.11)$ & $(=0.37)$ & $(=0.16)$ & $(=0.57)$ & $(=0.67)$ & $(=0.31)$ \\
\hline
\end{tabular}




\begin{tabular}{|c|c|c|c|c|}
\hline $\begin{array}{l}\text { Category } \\
\text { x Group }\end{array}$ & $\begin{array}{c}0.08 \\
(=0.78)\end{array}$ & $\begin{array}{c}0.74 \\
(=0.40)\end{array}$ & $\begin{array}{c}0.01 \\
(=0.94)\end{array}$ & $\begin{array}{c}0.71 \\
(=0.41)\end{array}$ \\
\hline $\begin{array}{l}\text { Category } \\
\text { x } \\
\text { Disease } \\
\text { duration }\end{array}$ & $\begin{array}{c}4.30 \\
(= \\
0.047)^{*}\end{array}$ & $\begin{array}{c}0.39 \\
(=0.54)\end{array}$ & $\begin{array}{c}0.02 \\
(=0.89)\end{array}$ & $\begin{array}{c}2.38 \\
(=0.13)\end{array}$ \\
\hline $\begin{array}{l}\text { Category } \\
\text { x LEDD }\end{array}$ & $\begin{array}{c}0.22 \\
(=0.64)\end{array}$ & $\begin{array}{c}3.29 \\
(=0.08)\end{array}$ & $\begin{array}{c}0.72 \\
(=0.40)\end{array}$ & $\begin{array}{c}0.04 \\
(=0.84)\end{array}$ \\
\hline
\end{tabular}

PD-nonVH, Parkinson patients without visual hallucinations $(\mathrm{N}=16)$;

PD-VH, Parkinson patients with visual hallucinations $(\mathrm{N}=16)$

RT, Recognition times; CVRT, Mean individual variability coefficients (individual SD/individual mean) of recognition times

Between-subjects factor: Group: 2 Levels: PD-nonVH, PD-VH

Within-subjects factors: Category: 2 levels: face, car

Covariates: Disease duration, levodopa-equivalent daily dose (LEDD)

${ }^{*} p<0.025$ Bonferroni corrected; ** $p<0.005$ Significant between and within main and interaction effects 
Supplementary Table S2.4. Mean performance measures of the study groups in the CFS condition

\begin{tabular}{llll}
\hline & HC & PD-nonVH & PD-VH \\
& $(\mathrm{n}=25)$ & $(\mathrm{n}=16)$ & $(\mathrm{n}=16)$ \\
\hline $\begin{array}{l}\text { Proportion Correct } \\
\text { Recognition }\end{array}$ & $0.84(0.04)$ & $0.86(0.03)$ & $0.61(0.08)$ \\
\hline Proportion Misses & $0.14(0.04)$ & $0.09(0.02)$ & $0.31(0.08)$ \\
\hline $\begin{array}{l}\text { Proportion Erroneous Object } \\
\text { Recognition in Scrambled }\end{array}$ & $0.08(0.04)$ & $0.08(0.06)$ & $0.21(0.07)$ \\
Images & & & \\
\hline Perceptual Error Score & $0.12(0.03)$ & $0.11(0.04)$ & $0.30(0.05)$ \\
\hline RT faces & $7.58(0.35)$ & $7.44(0.45)$ & $7.94(0.35)$ \\
\hline RT cars & $7.98(0.33)$ & $7.90(0.40)$ & $8.79(0.34)$ \\
\hline CVRT faces & $0.21(0.01)$ & $0.22(0.02)$ & $0.21(0.03)$ \\
\hline CVRT cars & $0.19(0.02)$ & $0.19(0.02)$ & $0.17(0.03)$ \\
\hline
\end{tabular}

Mean test values (S.E.M.) for each of the groups of the demographically matched sample. 25 Healthy controls (HC), 16 Parkinson patients without visual hallucinations (PD-nonVH), and 16 Parkinson patients with self-reported visual hallucinations (PD-VH). 
Supplementary Table S2.5. Results from ANOVA_1 in the CFS condition: Repeated Measures mixed ANOVA with the factors Category, Group and their interaction effects in the CFS condition

\begin{tabular}{|c|c|c|c|c|c|c|}
\hline \multirow[t]{2}{*}{ Factor } & $\begin{array}{c}\text { Proportion } \\
\text { Correct } \\
\text { Recogni } \\
\text { tion }\end{array}$ & $\begin{array}{l}\text { Proportion } \\
\text { Misses }\end{array}$ & $\begin{array}{l}\text { Proportion } \\
\text { Erroneous } \\
\text { Object } \\
\text { Recogni } \\
\text { tion in } \\
\text { Scrambled } \\
\text { Images }\end{array}$ & $\begin{array}{c}\text { Perceptual } \\
\text { Error } \\
\text { Score }\end{array}$ & CVRT & RT \\
\hline & $F(p)$ & $F(p)$ & $F(p)$ & $F(p)$ & $F(p)$ & $F(p)$ \\
\hline \multirow[t]{3}{*}{ Category } & 1.93 & 3.21 & & & 6.46 & 18.33 \\
\hline & $(=0.17)$ & $(=0.08)$ & & & $(=0.01)^{*}$ & $(=$ \\
\hline & & & & & & $0.00008)^{\star *}$ \\
\hline \multirow[t]{2}{*}{ Group } & 2.81 & 0.93 & 1.21 & 3.33 & 0.003 & 0.33 \\
\hline & $(=0.10)$ & $(=0.34)$ & $(=0.28)$ & $(=0.07)$ & $(=0.96)$ & $(=0.57)$ \\
\hline \multirow{2}{*}{$\begin{array}{l}\text { Category } \\
\text { x Group }\end{array}$} & 1.44 & 2.36 & & & 0.10 & 1.03 \\
\hline & $(=0.24)$ & $(=0.13)$ & & & $(=0.75)$ & $(=0.31)$ \\
\hline \multicolumn{7}{|c|}{ HC, Healthy controls $(\mathrm{N}=25)$; } \\
\hline \multicolumn{7}{|c|}{ PD, Parkinson patients with and without visual hallucinations $(N=32)$} \\
\hline \multicolumn{7}{|c|}{$\begin{array}{l}\text { RT, Recognition times; CVRT, Mean individual variability coefficients (individual SD/individual } \\
\text { mean) of recognition times }\end{array}$} \\
\hline \multicolumn{7}{|c|}{ Between-subjects factor: Group: 2 Levels: HC, PD } \\
\hline \multicolumn{7}{|c|}{ Within-subjects factors: Category: 2 levels: face, car } \\
\hline
\end{tabular}


Supplementary Table S2.6. Results from ANOVA_2 in the CFS condition: Repeated Measures mixed ANOVA with the factors Category, Group, their interaction effects in the CFS condition

\begin{tabular}{|c|c|c|c|c|c|c|}
\hline \multirow[t]{2}{*}{ Factor } & $\begin{array}{l}\text { Proportion } \\
\text { Correct } \\
\text { Recognition }\end{array}$ & $\begin{array}{l}\text { Proportio } \\
\text { n Misses }\end{array}$ & $\begin{array}{l}\text { Proportion } \\
\text { Erroneous } \\
\text { Object } \\
\text { Recogni } \\
\text { tion in } \\
\text { Scrambled } \\
\text { Images }\end{array}$ & $\begin{array}{c}\text { Perceptual } \\
\text { Error } \\
\text { Score }\end{array}$ & CVRT & RT \\
\hline & $F(p)$ & $F(p)$ & $F(p)$ & $F(p)$ & $F(p)$ & $F(p)$ \\
\hline \multirow[t]{2}{*}{ Category } & 0.03 & 0.05 & & & 3.36 & 12.18 \\
\hline & $(=0.87)$ & $(=0.82)$ & & & $(=0.08)$ & $(=0.002)^{\star *}$ \\
\hline \multirow[t]{2}{*}{ Group } & 8.18 & 7.01 & 2.04 & 8.27 & 0.37 & 1.78 \\
\hline & $(=0.008)^{*}$ & $(=0.01)^{*}$ & $(=0.16)$ & $(=0.007)^{*}$ & $(=0.55)$ & $(=0.19)$ \\
\hline \multirow{2}{*}{$\begin{array}{l}\text { Category x } \\
\text { Group }\end{array}$} & 10.65 & 7.32 & & & 0.05 & 1.01 \\
\hline & $(=0.003)^{* *}$ & $(=0.01)^{*}$ & & & $(=0.83)$ & $(=0.32)$ \\
\hline \multicolumn{7}{|c|}{ PD-nonVH, Parkinson patients without visual hallucinations $(\mathrm{N}=16)$; } \\
\hline \multicolumn{7}{|c|}{ PD-VH, Parkinson patients with visual hallucinations $(\mathrm{N}=16)$} \\
\hline \multicolumn{7}{|c|}{$\begin{array}{l}\text { RT, Recognition times; CVRT, Mean individual variability coefficients (individual SD/individual } \\
\text { mean) of recognition times }\end{array}$} \\
\hline \multicolumn{7}{|c|}{ Between-subjects factor: Group: 2 Levels: PD-nonVH, PD-VH } \\
\hline \multicolumn{7}{|c|}{ Within-subjects factors: Category: 2 levels: face, car } \\
\hline
\end{tabular}


Supplementary Table S2.7. Results from the Repeated Measures mixed ANCOVA in the CFS condition. Repeated Measures mixed ANCOVA with the factors Category, Group, their interaction effects and the covariates disease duration and LEDD in the CFS condition

\begin{tabular}{|c|c|c|c|c|c|c|}
\hline Factor & $\begin{array}{l}\text { Proportion } \\
\text { Correct } \\
\text { Recognition }\end{array}$ & $\begin{array}{l}\text { Proportion } \\
\text { Misses }\end{array}$ & $\begin{array}{l}\text { Proportion } \\
\text { Erroneous } \\
\text { Object } \\
\text { Recogni } \\
\text { tion in } \\
\text { Scrambled } \\
\text { Images }\end{array}$ & $\begin{array}{c}\text { Perceptual } \\
\text { Error } \\
\text { Score }\end{array}$ & CVRT & RT \\
\hline & $F(p)$ & $F(p)$ & $F(p)$ & $F(p)$ & $F(p)$ & $F(p)$ \\
\hline Category & $\begin{aligned} & 3.79 \\
&(=0.06)\end{aligned}$ & $\begin{aligned} & 3.71 \\
&(=0.06)\end{aligned}$ & & & $\begin{array}{c}0.33 \\
(=0.57)\end{array}$ & $\begin{array}{c}0.42 \\
(=0.52)\end{array}$ \\
\hline Group & $\begin{aligned} & 3.48 \\
&(=0.07)\end{aligned}$ & $\begin{array}{c}2.13 \\
(=0.16)\end{array}$ & $\begin{array}{c}5.67 \\
(=0.024)^{\star}\end{array}$ & $\begin{array}{c}7.66 \\
(=0.01)^{*}\end{array}$ & $\begin{array}{c}0.008 \\
(=0.93)\end{array}$ & $\begin{array}{c}0.02 \\
(=0.89)\end{array}$ \\
\hline $\begin{array}{l}\text { Disease } \\
\text { duration }\end{array}$ & $\begin{array}{c}1.17 \\
(=0.29)\end{array}$ & $\begin{array}{c}1.87 \\
(=0.18)\end{array}$ & $\begin{array}{c}0.01 \\
(=0.92)\end{array}$ & $\begin{array}{c}0.38 \\
(=0.55)\end{array}$ & $\begin{array}{c}1.04 \\
(=0.32)\end{array}$ & $\begin{array}{c}0.20 \\
(=0.66)\end{array}$ \\
\hline LEDD & $\begin{array}{l}1.61 \\
(0.22)\end{array}$ & $\begin{array}{c}3.45 \\
(=0.07)\end{array}$ & $\begin{array}{c}2.85 \\
(=0.10)\end{array}$ & $\begin{array}{c}0.08 \\
(=0.78)\end{array}$ & $\begin{array}{c}0.02 \\
(=0.89)\end{array}$ & $\begin{array}{c}2.50 \\
(=0.13)\end{array}$ \\
\hline $\begin{array}{l}\text { Category x } \\
\text { Group }\end{array}$ & $\begin{array}{l}2.24 \\
(0.14)\end{array}$ & $\begin{array}{c}1.11 \\
(=0.30)\end{array}$ & & & $\begin{array}{c}0.51 \\
(=0.48)\end{array}$ & $\begin{array}{c}0.07 \\
(=0.80)\end{array}$ \\
\hline $\begin{array}{l}\text { Category x } \\
\text { Disease } \\
\text { duration }\end{array}$ & $\begin{array}{c}0.04 \\
(=0.85)\end{array}$ & $\begin{array}{c}0.02 \\
(=0.90)\end{array}$ & & & $\begin{array}{c}0.28 \\
(=0.60)\end{array}$ & $\begin{array}{c}0.001 \\
(=0.98)\end{array}$ \\
\hline $\begin{array}{l}\text { Category x } \\
\text { LEDD }\end{array}$ & $\begin{array}{c}2.42 \\
(=0.13)\end{array}$ & $\begin{array}{c}2.40 \\
(=0.13)\end{array}$ & & & $\begin{array}{c}2.39 \\
(=0.13)\end{array}$ & $\begin{array}{c}0.51 \\
(=0.48)\end{array}$ \\
\hline
\end{tabular}

PD-nonVH, Parkinson patients without visual hallucinations $(\mathrm{N}=16)$; 
PD-VH, Parkinson patients with visual hallucinations $(\mathrm{N}=16)$

RT, Recognition times; CVRT, Mean individual variability coefficients (individual SD/individual mean) of recognition times

Between-subjects factor: Group: 2 Levels: PD-nonVH, PD-VH

Within-subjects factors: Category: 2 levels: face, car

Covariates: Disease duration, levodopa-equivalent daily dose (LEDD)

${ }^{*} p<0.05^{* *} p<0.005$ Significant between and within main and interaction effects 
Supplementary Table S2.8. Partial correlations between within and between network functional connectivity estimates and logarithmic transformation of the Perceptual Error Score in Parkinson patients without visual hallucinations (PD-nonVH) $(\mathbf{N}=6)$ and Parkinson patients with visual hallucinations (PD-VH) $(\mathrm{N}=10)$

\begin{tabular}{|c|c|c|c|c|c|c|c|c|}
\hline \multicolumn{9}{|c|}{ Log (Perceptual Error Score +1) } \\
\hline & DMN & DAN & SAL & I FP & r FP & SMN & $\begin{array}{l}\text { Visual } \\
\text { medial }\end{array}$ & $\begin{array}{l}\text { Visual } \\
\text { lateral }\end{array}$ \\
\hline & $r(p)$ & $r(p)$ & $r(p)$ & $r(p)$ & $r(p)$ & $r(p)$ & $r(p)$ & $r(p)$ \\
\hline DMN & $\begin{array}{l}<0.001 \\
(=0.99)\end{array}$ & $\begin{array}{l}0.32 \\
(=0.27)\end{array}$ & $\begin{array}{l}-0.06 \\
(=0.85)\end{array}$ & $\begin{array}{l}-0.01 \\
(=0.97)\end{array}$ & $\begin{array}{l}0.07 \\
(=0.82)\end{array}$ & $\begin{array}{l}0.45 \\
(=0.11)\end{array}$ & $\begin{array}{l}-0.08 \\
(=0.79)\end{array}$ & $\begin{array}{l}-0.03 \\
(=0.91)\end{array}$ \\
\hline DAN & $\begin{array}{l}0.29 \\
(=0.29)\end{array}$ & $\begin{array}{l}0.07 \\
(=0.82)\end{array}$ & $\begin{array}{l}-0.54 \\
(= \\
0.047)^{\star}\end{array}$ & $\begin{array}{l}0.46 \\
(=0.10)\end{array}$ & $\begin{array}{l}0.34 \\
(=0.23)\end{array}$ & $\begin{array}{l}-0.31 \\
(=0.28)\end{array}$ & $\begin{array}{l}0.34 \\
(=0.23)\end{array}$ & $\begin{array}{l}0.23 \\
(=0.44)\end{array}$ \\
\hline SAL & $\begin{array}{l}0.05 \\
(=0.86)\end{array}$ & $\begin{array}{l}0.03 \\
(=0.93)\end{array}$ & $\begin{array}{l}-0.21 \\
(=0.48)\end{array}$ & $\begin{array}{l}-0.14 \\
(=0.63)\end{array}$ & $\begin{array}{l}-0.24 \\
(=0.40)\end{array}$ & $\begin{array}{l}-0.25 \\
(=0.23)\end{array}$ & $\begin{array}{l}0.21 \\
(=0.48)\end{array}$ & $\begin{array}{l}0.20 \\
(=0.49)\end{array}$ \\
\hline I FP & $\begin{array}{l}-0.42 \\
(=0.14)\end{array}$ & $\begin{array}{l}-0.32 \\
(=0.27)\end{array}$ & $\begin{array}{l}-0.35 \\
(=0.22)\end{array}$ & $\begin{array}{l}-0.03 \\
(=0.91)\end{array}$ & $\begin{array}{l}-0.02 \\
(=0.95)\end{array}$ & $\begin{array}{l}-0.51 \\
(=0.06)\end{array}$ & $\begin{array}{l}-0.27 \\
(=0.35)\end{array}$ & $\begin{array}{l}-0.16 \\
(=0.58)\end{array}$ \\
\hline$r$ FP & $\begin{array}{l}0.02 \\
(=0.96)\end{array}$ & $\begin{array}{l}-0.36 \\
(=0.21)\end{array}$ & $\begin{array}{l}0.44 \\
(=0.12)\end{array}$ & $\begin{array}{l}<0.001 \\
(=0.99)\end{array}$ & $\begin{array}{l}-0.41 \\
(=0.15)\end{array}$ & $\begin{array}{l}-0.12 \\
(=0.72)\end{array}$ & $\begin{array}{l}0.17 \\
(=0.57)\end{array}$ & $\begin{array}{l}-0.44 \\
(=0.12)\end{array}$ \\
\hline SMN & $\begin{array}{l}0.49 \\
(=0.08)\end{array}$ & $\begin{array}{l}-0.20 \\
(=0.49)\end{array}$ & $\begin{array}{l}0.08 \\
(=0.78)\end{array}$ & $\begin{array}{l}-0.19 \\
(=0.52)\end{array}$ & $\begin{array}{l}-0.32 \\
(=0.27)\end{array}$ & $\begin{array}{l}-0.09 \\
(=0.74)\end{array}$ & $\begin{array}{l}-0.17 \\
(=0.56)\end{array}$ & $\begin{array}{l}0.02 \\
(=0.95)\end{array}$ \\
\hline $\begin{array}{l}\text { Visual } \\
\text { medial }\end{array}$ & $\begin{array}{l}-0.07 \\
(=0.82)\end{array}$ & $\begin{array}{l}0.48 \\
(=0.08)\end{array}$ & $\begin{array}{l}-0.29 \\
(=0.32)\end{array}$ & $\begin{array}{l}0.15 \\
(=0.60)\end{array}$ & $\begin{array}{l}0.09 \\
(=0.76)\end{array}$ & $\begin{array}{l}-0.23 \\
(=0.44)\end{array}$ & $\begin{array}{l}0.16 \\
(=0.58)\end{array}$ & $\begin{array}{l}-0.03 \\
(=0.91)\end{array}$ \\
\hline $\begin{array}{l}\text { Visual } \\
\text { lateral }\end{array}$ & $\begin{array}{l}0.20 \\
(=0.49)\end{array}$ & $\begin{array}{l}0.34 \\
(=0.23)\end{array}$ & $\begin{array}{l}0.29 \\
(=0.31)\end{array}$ & $\begin{array}{l}0.11 \\
(=0.71)\end{array}$ & $\begin{array}{l}0.04 \\
(=0.88)\end{array}$ & $\begin{array}{l}-0.04 \\
(=0.89)\end{array}$ & $\begin{array}{l}-0.18 \\
(=0.53)\end{array}$ & $\begin{array}{l}-0.09 \\
(=0.74)\end{array}$ \\
\hline
\end{tabular}

\section{CVRT}

\begin{tabular}{lllllllll}
\hline & DMN & DAN & SAL & I FP & r FP & SMN & $\begin{array}{l}\text { Visual } \\
\text { medial }\end{array}$ & $\begin{array}{l}\text { Visual } \\
\text { lateral }\end{array}$ \\
\hline DMN & 0.19 & -0.06 & 0.08 & -0.35 & -0.29 & -0.18 & 0.02 & 0.07 \\
& $(=0.52)$ & $(=0.83)$ & $(=0.79)$ & $(=0.22)$ & $(=0.30)$ & $(=0.54)$ & $(=0.94)$ & $(=0.82)$ \\
\hline
\end{tabular}




\begin{tabular}{|c|c|c|c|c|c|c|c|c|}
\hline DAN & $\begin{array}{l}0.47 \\
(=0.09)\end{array}$ & $\begin{array}{l}-0.20 \\
(=0.49)\end{array}$ & $\begin{array}{l}0.12 \\
(=0.69)\end{array}$ & $\begin{array}{l}0.40 \\
(=0.15)\end{array}$ & $\begin{array}{l}0.31 \\
(=0.28)\end{array}$ & $\begin{array}{l}0.21 \\
(=0.48)\end{array}$ & $\begin{array}{l}0.09 \\
(=0.75)\end{array}$ & $\begin{array}{l}0.51 \\
(=0.06)\end{array}$ \\
\hline SAL & $\begin{array}{l}-0.29 \\
(=0.32)\end{array}$ & $\begin{array}{l}-0.49 \\
(=0.07)\end{array}$ & $\begin{array}{l}-0.01 \\
(=0.97)\end{array}$ & $\begin{array}{l}-0.15 \\
(=0.62)\end{array}$ & $\begin{array}{l}-0.30 \\
(=0.29)\end{array}$ & $\begin{array}{l}-0.31 \\
(=0.28)\end{array}$ & $\begin{array}{l}-0.24 \\
(=0.42)\end{array}$ & $\begin{array}{l}-0.29 \\
(=0.31)\end{array}$ \\
\hline I FP & $\begin{array}{l}0.05 \\
(=0.86)\end{array}$ & $\begin{array}{l}-0.14 \\
(=0.64)\end{array}$ & $\begin{array}{l}-0.002 \\
(=0.99)\end{array}$ & $\begin{array}{l}0.39 \\
(=0.17)\end{array}$ & $\begin{array}{l}0.26 \\
(=0.38)\end{array}$ & $\begin{array}{l}0.03 \\
(=0.93)\end{array}$ & $\begin{array}{l}-0.23 \\
(=0.43)\end{array}$ & $\begin{array}{l}-0.04 \\
(=0.89)\end{array}$ \\
\hline rFP & $\begin{array}{l}-0.53 \\
(= \\
0.053)\end{array}$ & $\begin{array}{l}0.01 \\
(=0.97)\end{array}$ & $\begin{array}{l}0.38 \\
(=0.19)\end{array}$ & $\begin{array}{l}-0.28 \\
(=0.34)\end{array}$ & $\begin{array}{l}-0.22 \\
(=0.44)\end{array}$ & $\begin{array}{l}-0.21 \\
(=0.47)\end{array}$ & $\begin{array}{l}-0.16 \\
(=0.59)\end{array}$ & $\begin{array}{l}0.22 \\
(=0.46)\end{array}$ \\
\hline SMN & $\begin{array}{l}-0.22 \\
(=0.45)\end{array}$ & $\begin{array}{l}-0.08 \\
(=0.79)\end{array}$ & $\begin{array}{l}0.32 \\
(=0.27)\end{array}$ & $\begin{array}{l}-0.08 \\
(=0.78)\end{array}$ & $\begin{array}{l}-0.55 \\
(= \\
0.04)^{\star}\end{array}$ & $\begin{array}{l}0.33 \\
(=0.25)\end{array}$ & $\begin{array}{l}-0.18 \\
(=0.53)\end{array}$ & $\begin{array}{l}0.14 \\
(=0.63)\end{array}$ \\
\hline $\begin{array}{l}\text { Visual } \\
\text { medial }\end{array}$ & $\begin{array}{l}-0.05 \\
(=0.87)\end{array}$ & $\begin{array}{l}0.09 \\
(=0.76)\end{array}$ & $\begin{array}{l}-0.19 \\
(=0.52)\end{array}$ & $\begin{array}{l}-0.18 \\
(=0.55)\end{array}$ & $\begin{array}{l}-0.27 \\
(=0.36)\end{array}$ & $\begin{array}{l}0.08 \\
(=0.79)\end{array}$ & $\begin{array}{l}-0.16 \\
(=0.59)\end{array}$ & $\begin{array}{l}-0.02 \\
(=0.96)\end{array}$ \\
\hline $\begin{array}{l}\text { Visual } \\
\text { lateral }\end{array}$ & $\begin{array}{l}-0.002 \\
(=0.99)\end{array}$ & $\begin{array}{l}0.27 \\
(=0.34)\end{array}$ & $\begin{array}{l}0.18 \\
(=0.54)\end{array}$ & $\begin{array}{l}0.36 \\
(=0.21)\end{array}$ & $\begin{array}{l}0.16 \\
(=0.59)\end{array}$ & $\begin{array}{l}0.18 \\
(=0.55)\end{array}$ & $\begin{array}{l}0.10 \\
(=0.73)\end{array}$ & $\begin{array}{l}-0.03 \\
(=0.93)\end{array}$ \\
\hline
\end{tabular}

Partial correlations corrected for Disease duration and LEDD of hallucinating and non-hallucinating Parkinson patients $(n=16) .{ }^{*} p<0.05$ 


\section{References}

Carmel, D., Arcaro, M., Kastner, S., \& Hasson, U. (2010). How to create and use binocular rivalry. Journal of Visualized Experiments: JoVE, (45). https://doi.org/10.3791/2030

Ekman, P., \& Friesen, W. V. (1976). Pictures of Facial Affect. Palo Alto.CA.Colsulting Psychologists Press.

Stein, T., Hebart, M. N., \& Sterzer, P. (2011). Breaking Continuous Flash Suppression: A New Measure of Unconscious Processing during Interocular Suppression? Frontiers in Human Neuroscience, 5, 167. https://doi.org/10.3389/fnhum.2011.00167

Tsuchiya, N., \& Koch, C. (2005). Continuous flash suppression reduces negative afterimages. Nature Neuroscience, 8(8), 1096-1101. https://doi.org/10.1038/nn1500 


\section{Chapter 3}

\section{Behavioral, Neural and Heart Rate Variability and their Relation to Cognitive Performance in Healthy Young and Healthy Elderly}

Kristina Miloserdov ${ }^{1,3,4}$, Carsten Schmidt-Samoa ${ }^{1}$, Diana Toscano ${ }^{1}$, Iris Steinmann ${ }^{1}$, Annekathrin Schacht ${ }^{4,5}$, Melanie Wilke $e^{1,2,3,4}$

${ }^{1}$ Institute of Cognitive Neurology, University Medical Center Goettingen, Robert-KochStr. 40, Goettingen, 37075, Germany

${ }^{2}$ German Primate Center, Leibniz Institute for Primate Research, Kellnerweg 4, Goettingen, 37077, Germany

${ }^{3}$ DFG Center for Nanoscale Microscopy \& Molecular Physiology of the Brain (CNMPB)

${ }^{4}$ Leibniz-Science Campus Primate Cognition, German Primate Center, Goettingen, Germany

${ }^{5}$ Affective Neuroscience and Psychophysiology Laboratory, University of Goettingen, Goßlerstr. 14, Germany

\section{Keywords:}

Aging, trial-by-trial variability, fluctuations, heart rate variability, variability quenching 


\section{Abstract}

Behavioral variability is a marker of cognitive decline in different clinical and non-clinical groups such as healthy elderly (Bielak et al., 2010; Gorus et al., 2008; S. W. MacDonald, Nyberg, \& Bäckman, 2006), Alzheimer's disease (Gorus et al., 2008), neurological dysfunction related to lethal prognosis (Macdonald, Hultsch, \& Dixon, 2008) and Parkinson's disease (Miloserdov et al., submitted). Mechanisms and sources of behavioral variability are still not fully understood and might be explained by neural processes (MacDonald et al., 2009). High behavioral and neural variability is related to cognitive impairment and dementia (Bielak et al., 2010; Dinstein et al., 2015), in contrast to heart rate variability (HRV) which is related to higher cognitive functioning (Schaffer et al., 2014). To improve understanding of intra-individual variability we investigated age related changes in cognition in combination with psychophysical, cardiac and neural measurements. We tested whether healthy elderly show lower cognitive performance, higher behavioral variability, increased neural variability, increased theta power and reduced HRV. Moreover, we investigated whether lower cognitive functioning is related to behavioral, neural, cardiac variability and other possible marker of cognitive decline such as P1, P3 and changes in power of different frequency bands. Healthy elderly show more behavioral variability in response to cars, reduced P1 and P3 amplitude, higher change of theta power and lower cardiac variability compared to healthy young. Moreover, we could show that lower cognitive performance is related to higher behavioral variability and lower neural variability in healthy elderly. Behavioral and neural variability might be a possible marker of cognitive decline. 


\subsection{Introduction}

Behavioral variability can reflect optimal cognitive state while optimizing performance in a new complex task or suboptimal cognitive state by trying to hold the performance level in a familiar task (Li, Huxhold, \& Schmiedek, 2004). The mechanisms of behavioral variability and their relation to neural variability are not fully understood. Neural variability might arise from different sources and pursue different aims. Adaptive variability serves to reach a goal using different strategies such as plasticity (to master large learning gains), diversity (explore different strategies to meet task complexity) and adaptability (to keep up optimal performance due to task challenges). While adaptive variability results from facing new challenges or improve achievements, maladaptive variability fluctuates in performance to master an already achieved performance level without additional challenges indicating a deficit in robustness ( $\mathrm{Li}$, Huxhold, \& Schmiedek, 2004). Higher intra-individual variability in healthy elderly is related to reduced memory function (Papenberg et al., 2011). Intra-individual variability reflects age related cognitive decline (Bielak et al., 2010; Gorus et al., 2008; MacDonald et al., 2006). Intra-individual variability in a complex task predicts mild cognitive impairment $(\mathrm{MCl})$ and status of Alzheimers disease (Gorus et al., 2008) and is increased in hallucinating PD patients (Miloserdov et al., submitted). Moreover, intra-individual variability is an early marker of neurological dysfunction related to lethal prognosis (Macdonald et al., 2008). Mechanisms and sources of behavioral variability is still a challenging subject and might be explained by neural processes (MacDonald et al., 2009). 


\subsubsection{Neural variability}

Neural processes underlying perception and cognition can be reflected by different measurements such as event-related potentials indicating early, perceptual and late, cognitive processes. Healthy elderly show changes in an early, perceptual component such as the P1 and late, cognitive component such as the P3. In healthy elderly P1 amplitude is reduced and double peak occurs more often compared to healthy young adults (Stothart, Tales, Hedge, \& Kazanina, 2014). In healthy elderly decreased P3 is related to working memory performance (Lubitz, Niedeggen, \& Feser, 2017). P3 intertrial variability is related to more severe cognitive symptoms in schizophrenia (Kim et al., 2018). Behavioral variability might be reflected by changes in temporal processing of information reflected in P3 reduction and variability. Another measurement of neural variability is variability quenching, the reduction of neural variability after stimulus presentation (Arazi, Censor, et al., 2017). In healthy young adults increased variability quenching, reduced neural variability, was related to better task performance (Arazi, Censor, et al., 2017). Older and slower performing adults showed less changes in brain variability (Garrett et al., 2013) indicating its potential as marker of cognitive changes during aging. Neural variability magnitudes are stable over time and test conditions in healthy young human volunteers (Arazi, Gonen-Yaacovi, et al., 2017) showing its reliability as a possible marker of cognitive changes.

\subsubsection{Time frequency subtypes and cognitive performance}

Different frequency bands are associated with different functions like gamma with visual perception, attention and memory (Jensen, Hougaard, Amin, Larsson, \& Ashina, 2015), beta with motor function (He, Zhang, Chen, Xie, Gan, Yang, et al., 2017), alpha and 
theta with cognition (Caviness et al., 2007). Changes in alpha and theta power might reflect changes in cognitive function. Individuals with $\mathrm{MCl}$ are characterized by reduced power in alpha and beta frequency and increased delta and theta power indicating the potential of these frequency bands as possible markers of cognitive decline (López et al., 2014). Theta oscillations are related to cognitive load, working memory, selective attention and cognitive integration (Givens, 1996; Vertes, 2005). Older adults show stronger theta power engaged in visual and audiovisual detection task in frontal brain regions in contrast to younger volunteers showing stronger theta power in central regions (Yan et al., 2016). Theta power is related to better cognitive performance such as memory and attention in frontal brain regions of healthy elderly. Moreover, increased theta power correlates with reduced reaction time variability on the sustained attention task in frontal electrodes of healthy elderly (Finnigan \& Robertson, 2011). Furthermore, healthy elderly show increased theta power compared to elderly with $\mathrm{MCl}$ (Cummins, Broughton, \& Finnigan, 2008) and PD patients with $\mathrm{MCl}$ (He, Zhang, Chen, Xie, Gan, Wang, et al., 2017). In addition, transition from $\mathrm{MCl}$ to Alzheimer's disease is characterized by lower theta power in temporal, occipital, parietal and frontal electrodes indicating increased theta power as a marker of cognitive functioning (Mazaheri et al., 2018). However, resting state EEG shows global increase in theta power in AD and increase in theta power is related to worse cognitive performance in $\mathrm{MCl}$ and $\mathrm{PD}$ indicating higher levels of theta power as an possible early marker of cognitive decline (He, Zhang, Chen, Xie, Gan, Wang, et al., 2017; Musaeus et al., 2018). At first glance cognitive changes, differences in spectral frequency power and neural variability might provide explanations about behavioral variability. However, other regulatory systems, 
such as the parasympathetic nervous system might play a role in changes of behavioral variability.

\subsubsection{Heart rate variability}

The parasympathetic nervous system is important for self-regulation which is related to cognition, emotion and health. HRV is the difference in time intervals between heartbeats which is part of the autonomous regulatory systems that is necessary to master challenges and optimize performance (Shaffer et al., 2014). Improvement of self-regulation involving $\mathrm{HRV}$ can be achieved by the heart and brain network. The heart receives input from the parasympathetic nervous system (Chapleau \& Sabharwal, 2011). Different theories discuss possible mechanisms of this connection and the role of HRV in this network. Porge's polyvalgal theory assumes that the vagal nerve which can slow down the heart rate, contain specialized subsystems for regulation of adaptive responses. The older unmyelinated nerves regulate freezing behavioral responses and the newer myelinated nerves originating from the nucleus ambiguus, are important for self-regulatory ability by inhibiting sympathetic outflow to the heart (Shaffer et al., 2014). Increased vagal tone is related to better social functioning (Porges, 2007). The neurovisceral integration model assumes a connection between the heart and the prefrontal cortex through the central autonomic network and the vagus (Thayer, Hansen, Saus-Rose, \& Johnsen, 2009). Higher vagal tone reflects better executive cognitive performance, emotional and health regulation (Thayer, Hansen, Saus-Rose, \& Johnsen, 2009). HRV is regulated by connections between amydgala and medial prefrontal cortex. HRV is linked to executive functions, reflecting the functional capacity of the brain to support working memory and self-regulations. It is hypothesized that to 
inhibit unpleasant memories the central autonomic network could decrease prefrontal activation, increase heart rate and reduce HRV (Shaffer et al., 2014). McCraty and Childre's Psychophysiological coherence model proposes that time intervals between the heart beats encode information which helps synchronize multiple systems, emphasising the afferent pathways carrying input from the heart to the brain (Shaffer et al., 2014). HRV decreases with age (for review see (Shaffer et al., 2014). Cognitive functioning is related to increased rest HRV (Thayer et al., 2009). HRV might not only play a role for cognitive changes related to aging (Shaffer et al., 2014) as in healthy young volunteers HRV varies as function of demands in sustained attention (LuqueCasado, Perales, Cárdenas, \& Sanabria, 2016). Moreover, regulation of the outonomic outflow of the heart might be affected by neurodegenerative changes in Parkinson and dementia. HRV as a marker of parasympathetic activity and it is related to increased risk developing PD but not other measures of cardiac autonomic function including frequency-domain measurements (Alonso, Huang, Mosley, Heiss, \& Chen, 2015). HRV is an index of self-regulatory control, greater resting HRV is related to better performance of executive functions (Shaffer et al., 2014). High behavioral and neural variability is related to cognitive impairment and dementia (Bielak et al., 2010; Dinstein, Heeger, \& Behrmann, 2015) and HRV to improved cognitive functioning (Shaffer et al., 2014).

Intra-individual variability and its neural correlates might provide a marker for different profiles of Parkinson symptoms. Improvements in understanding mechanisms and sources of intra-individual variability might promote differential diagnosis and maybe even more individualized treatment. To improve understanding of intra-individual variability we investigated age related changes in cognition in combination with 
psychophysical, cardiac and neural measurements. This might provide new insights into behavioral, neural and cardiac variability and its relation to age related cognitive changes. We expect that healthy elderly show lower cognitive performance, higher behavioral variability, increased neural variability, increased theta power and reduced HRV. Moreover, we expect that lower cognitive functioning is related to higher theta power, higher behavioral and neural variability and lower HRV.

\subsection{Materials and Methods}

\subsubsection{Participants}

Forty-eight healthy volunteers (26 Healthy Elderly) and (22 Healthy Young) without history of neurological or psychiatric diseases were recruited. ECG of 24 older participants and 20 younger participants were used (in two subjects ECG was not recorded and in two subjects data quality was not sufficient for further analyses). Two Healthy Elderly participants did not follow the instructions and were excluded from further analyses. All participants signed an informed consent form prior to the examination. Exclusion criteria were moderate to severe general cognitive impairment/dementia (Mini Mental State Examination (MMSE) score < 26) (Folstein, Folstein, \& McHugh, 1975), visual acuity below 80\% (Poster Radler 2010). The Healthy Young and Healthy Elderly did not differ on demographic variables such as years of education $\left(\mathrm{t}(46)=1.27, \mathrm{p}=0.21\right.$, gender $\left(\mathrm{X}^{2}=0.95, \mathrm{p}=0.76\right)$ and handedness $\left(\mathrm{X}^{2}=\right.$ $0.34, p=0.84$ ) assessed by Edinburgh Handedness Inventory (Table 3.1). Given that depression is less prevalent in older adults compared to younger adults we measured depressive symptoms using Beck Depression Inventory (Beck, Ward, Mendelson, 
Mock, \& Erbaugh, 1961). However, the lower BDI scores in Healthy Elderly were not significant (Kessler et al., 2010) (Table 3.1). 
Table 3.1. Demographic and cognitive characteristics of Healthy Young and Healthy Elderly.

\begin{tabular}{|c|c|c|c|}
\hline & $\begin{array}{l}\text { Young } \\
(N=22)\end{array}$ & $\begin{array}{l}\text { Elderly } \\
(\mathrm{N}=24)\end{array}$ & P- value \\
\hline & Mean (SD) & Mean (SD) & \\
\hline Age (years) ${ }^{b}$ & $24.77(3.90)$ & $63.25(7.98)$ & $<0.00001^{*}$ \\
\hline Gender (female/male) ${ }^{\mathrm{d}}, \mathrm{n}(\%)$ & $12 / 10(55 \% / 45 \%)$ & $12 / 12(50 \% / 50 \%)$ & $=0.75$ \\
\hline Education years ${ }^{b}$ & $17.27(2.75)$ & $15.92(4.33)$ & $=0.21$ \\
\hline MMSE $^{\mathrm{C}}$ & $29.32(0.99)$ & $29.46(0.72)$ & $=0.81$ \\
\hline Trail A (sec.) ${ }^{c}$ & $24.74(7.66)$ & $34.36(11.12)$ & $=0.0003^{* *}$ \\
\hline Trail B (sec.) ${ }^{c}$ & $56.73(20.72)$ & 76.23 (33.32) & $=0.02^{*}$ \\
\hline Verbal Fluency $^{\mathrm{a}}$ & $28.05(7.52)$ & $27.18(6.32)$ & $=0.69$ \\
\hline Stroop Test RT contgruent (ms) ${ }^{a}$ & $987,67(165.22)$ & $1361.756(257.84)$ & $<0.001^{\star \star}$ \\
\hline $\begin{array}{l}\text { Stroop Test RT noncongruent } \\
(\mathrm{ms})^{\mathrm{c}}\end{array}$ & $1059.72(200.49)$ & $1584.93(415.61)$ & $<0.001^{\star *}$ \\
\hline Stroop Test CVRT congruent ${ }^{c}$ & $31.83(12.21)$ & $38.46(13.44)$ & $=0.06$ \\
\hline Stroop Test CVRT noncongruent ${ }^{c}$ & $28.88(8.27)$ & $32.97(10.87)$ & $=0.32$ \\
\hline Simple RT $(\mathrm{ms})^{\mathrm{c}}$ & $243.22(37.52)$ & $278.08(49.65)$ & $=0.006^{*}$ \\
\hline Simple RT CVRT ${ }^{\mathrm{c}}$ & $0.34(0.19)$ & $0.41(0.21)$ & $=0.09$ \\
\hline Taprequency $^{c}$ (tap/sec.) & $2.19(1.15)$ & $2.12(0.63)$ & $=0.47$ \\
\hline Tapfrequency CVRT ${ }^{\mathrm{C}}$ & $10.37(8.26)$ & $9.50(3.29)$ & $=0.73$ \\
\hline $\mathrm{BDI}^{\mathrm{a}}$ & $5.10(3.48)$ & $3.30(3.66)$ & $=0.10$ \\
\hline $\begin{array}{l}\text { Mars Letters Contrast Sensitivity } \\
\text { Test }^{c}\end{array}$ & $1.81(0.04)$ & $1.79(0.04)$ & $=0.35$ \\
\hline $\begin{array}{l}\text { Handedness (right/left / } \\
\text { ambidexter) }{ }^{d}, \mathrm{n}(\%)\end{array}$ & $\begin{array}{l}18 / 3 / 1 \\
(82 \% / 14 \% / 4 \%)\end{array}$ & $\begin{array}{l}21 / 2 / 1 \\
(88 \% / 8 \% / 4 \%)\end{array}$ & $=0.84$ \\
\hline \multicolumn{4}{|c|}{${ }^{a}$ t-test; ${ }^{b}$ t-test with unequal variances ${ }^{c}$ Mann-Whitney $U$ test; ${ }^{d}$ Chi-square -test; } \\
\hline \multicolumn{4}{|c|}{ MMSE, Mini Mental State Examination; BDI, Beck's, Depression Inventory; } \\
\hline \multicolumn{4}{|c|}{ RT, Recognition times, CVRT, Coefficient of Recognition time variability } \\
\hline${ }^{*} p<0.05{ }^{* *} p<0.005$ & & & \\
\hline
\end{tabular}




\subsubsection{Neuropsychological Testing}

Contrast sensitivity was evaluated with the Mars Letters Test (Mars Perceptrix Corporation, Chappaqua, NY, USA). Participants filled in a self-report depression scale (Beck's Depression Inventory, BDI) (Beck, Ward, Mendelson, Mock, \& Erbaugh, 1961b). Different domains of cognitive functioning are reduced in healthy elderly (Hedden \& Gabrieli, 2004). To asses these cognitive differences we used the Trail Making Test (TMT) (Reitan, \& Wolfson, 1993), Verbal Fluency test (Troyer, Moscovitch, \& Winocur, 1997) and the Stroop test (Stroop, 1935). To test motor differences in rhythm formation we used the Finger Tapping Test (Arias, Robles-García, Espinosa, Corral, \& Cudeiro, 2012) where participants were instructed to press a button with the index finger of their dominant hand at a comfortable rate (speed of their choice), and to try to maintain a constant interval between each of their button presses. Subjects performed 3 sets of 53 button presses. Moreover, to test age differences in simple reaction time variability we designed a Simple Reaction Time test in which participants were asked to press a button with the index finger of their dominant hand as soon as they saw a cue on the screen. The task consisted of 50 trials, each separated by a random interval ranging from 0.5 to 1.5 seconds.

\subsubsection{Image Recognition Task}

Participants were placed in a dark room and asked to lay their heads in a chin-rest to limit head movements. The chin-rest was placed at a distance of $57 \mathrm{~cm}$ from the computer screen. Participants had to identify different images presented at a high and low contrast level while their brain activity was recorded using EEG. The task was designed based on the results of our previous study which revealed higher recognition 
time variability in hallucinating PD patients compared to non-hallucinating PD patient where the contrast of the stimulus was stepped up continuously (Miloserdov et al., submitted). Using high and low contrast level we were aiming to investigate whether high or low contrast stimuli contribute more to recognition time variability.

\subsubsection{Stimuli}

The stimuli were presented with a custom script using Matlab version R2011b and the Psychtoolbox (Brainard, 1997) on a 27 inch LCD monitor with a screen resolution of $1920 \times 1080$ pixels and a vertical refresh rate of $60 \mathrm{~Hz}$. The stimuli were photographs of three different objects: cars, faces and scrambled images (Figure 3.1A). Images had a size of $480 \times 480$ pixels ( $15^{\circ}$ of visual angle), and were presented at the center of the screen. The photographs of the cars were obtained from the Caltech image archive (http://vision.caltech.edu/archive.html), and the photographs of faces, from the Paul Ekman Group picture bank (Ekman \& Friesen, 1976). We presented 10 different faces and 10 different cars. It is important to mention that all the faces presented had a neutral expression. Scrambled images were created dividing the images of faces and cars into $10 \times 10$ pixels blocks and randomizing their position using Matlab (Figure

3.1A). The three types of stimuli were shown at $100 \%$ and $10 \%$ contrast, giving us a total of six different conditions (i.e. cars at $100 \%$ and $10 \%$ contrast, faces at $100 \%$ and $10 \%$ contrast, and scrambled images at $100 \%$ and $10 \%$ contrast). 
A

\section{Examples target images by category}
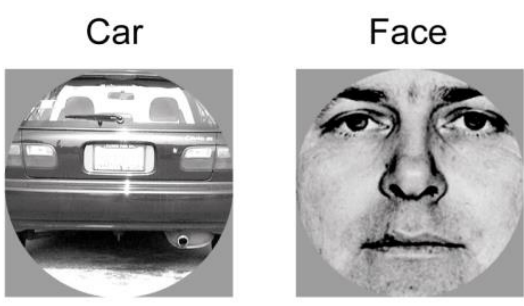

Scrambled

Contrast

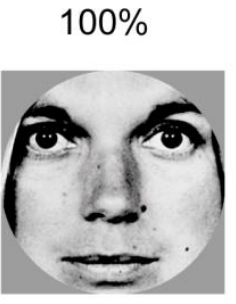

$10 \%$

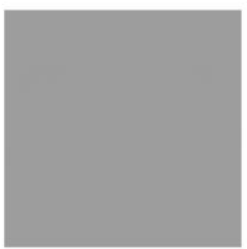

B

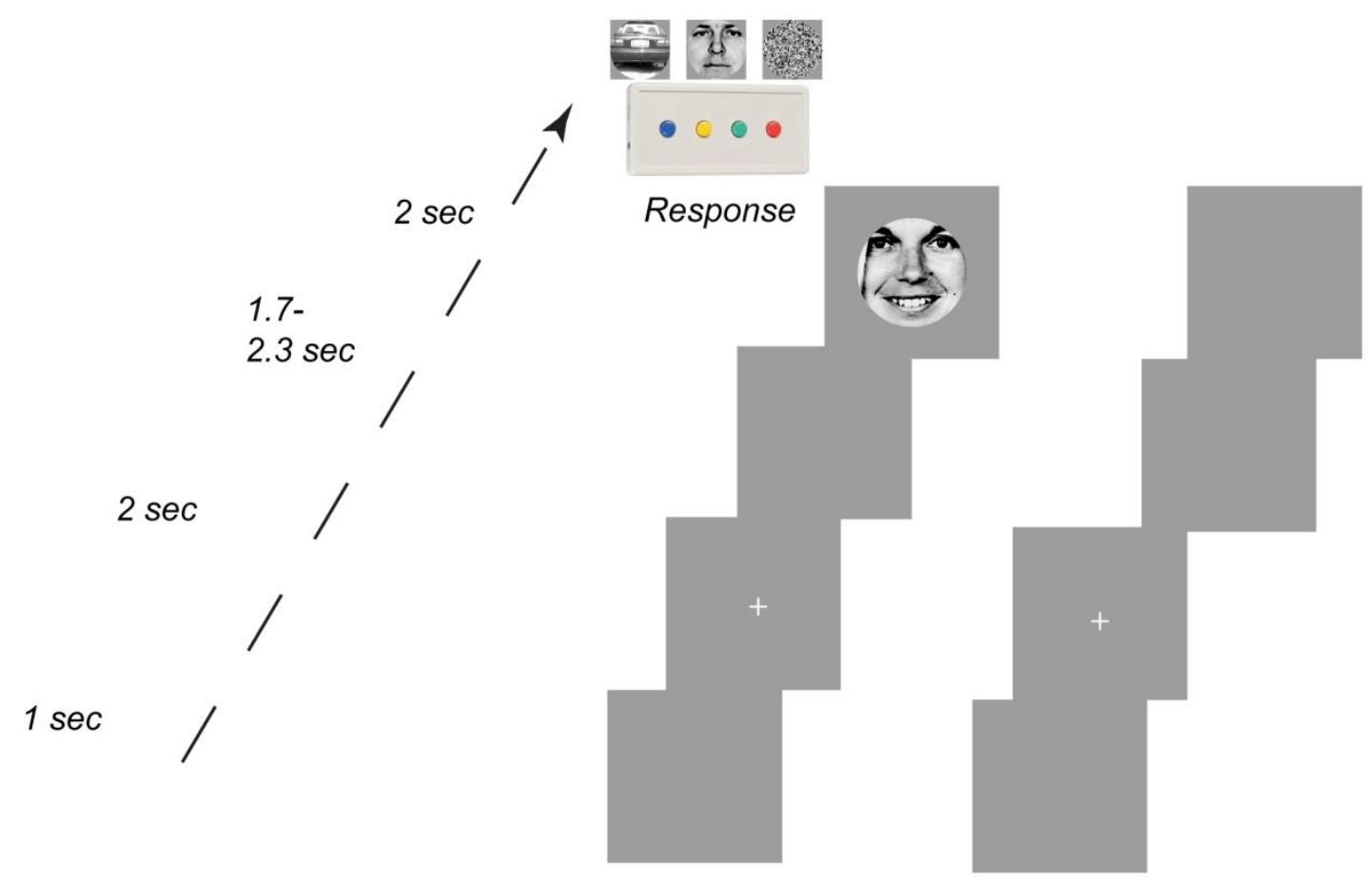

Figure 3.1. Task design and trial structure. A) Example images of face, car and scrambled images used in the experiments B) Trial sequence. 


\subsubsection{Trial Sturcture}

Each trial (Figure 3.1B) started with prefixation of 1 second, during which a black screen was shown. Prefixation was followed by a white fixation cross on a black background presented for 2 seconds in the center of the screen. After the fixation period, a jitter between 1.7 to 2.3 seconds preceded the presentation of the stimulus, which was presented for 2 seconds. The participants were instructed to give their answer as soon as possible. They had to categorize the object on the screen (either car, face or scrambled image, at $10 \%$ or $100 \%$ contrast), giving their answers with a button-box using their index, middle and ring fingers (participants were told which button corresponded to which object before starting the task). To avoid biases in the reaction times of a particular stimulus that could be caused by differences in the speed of a particular finger (Wilimzig, Ragert, \& Dinse, 2012), we assigned one of three different possible button orders to each participant. Regardless of the time it took participants to respond, the stimulus remained on the screen for 2 seconds. After their response, they were prompted to confirm their answer. If the participants did not reply within the 2 seconds of the presentation of the stimulus, they were asked to guess which object was presented on the screen. After their response, the next prefixation period would start. The task was structured in a block manner. The participants had to complete a total of 8 blocks. Each block consisted of a total of 60 trials. In every block, each condition was presented ten times in a pseudo-randomized manner, giving us a total of 80 trials per condition and 480 trials in total at the end of the test. 


\subsubsection{EEG Recording and Preprocessing}

EEG data was recorded at a sampling rate of $1000 \mathrm{~Hz}$ using a 64-channel Brain Products system elastic cap. The cap includes a reference electrode located at FCz. The FieldTrip toolbox for Matlab (Oostenveld, Fries, Maris, \& Schoffelen, 2011) was used for processing the data. Continuous EEG data was segmented into $3000 \mathrm{~ms}$ long epochs (1500 ms prestimulus to $1500 \mathrm{~ms}$ poststimulus). Data was filtered offline using a 0.1-220 band-pass filter (butterworth, hamming window) and a $50 \mathrm{~Hz}$ notch filter. Jumps and clips were interpolated and trials containing muscle artifacts were rejected. Blink artifacts were corrected using Independent Component Analysis (ICA). Data was rereferenced to the common average. Given that evidence suggests that face processing is associated with cognitive performance (Feuerriegel, Churches, Hofmann, \& Keage, 2015; Saavedra, Olivares, \& Iglesias, 2012) we focused our analyses on the electrodes near the anatomical regions associated with face processing (TP10, TP8, PO8, P8, TP9, TP7, P7, PO7 electrodes, which include the middle/posterior fusiform gyrus and the posterior superior temporal sulcus (Jonas et al., 2016). All analyses were performed separately for each of the stimuli (cars, faces and scrambled images), as well as each contrast level (100\% and $10 \%)$.

\subsubsection{Time domain analysis}

For the time domain analysis, data was filtered using a low pass filter of $40 \mathrm{~Hz}$ (butterworth, hamming window). 


\subsubsection{Event Related Potentials}

To evaluate the event related potentials (ERP) a baseline of - 200 to $0 \mathrm{~ms}$ before stimulus presentation was used and the mean amplitude for the ERP of interest, P1 and P3, was calculated. Given that early visual processes are expected to be reflected in V1 we calculated the mean amplitude of the P1 component for each group over the $\mathrm{O} 1$ and $\mathrm{O} 2$ channels using a time window of 70 to $130 \mathrm{~ms}$ after stimulus presentation (Wykowska \& Schubö, 2012). Given that parietal P3 is sensitive for perceptual difference between stimulus types (Harper, Malone, \& lacono, 2017) we derived the signal of P3, P4 electrodes for calculation of the area of the amplitude of P3 using a time window of 400 to $620 \mathrm{~ms}$ after stimulus presentation (Salti, Bar-Haim, \& Lamy, 2012) which is sensitive to changes in amplitude in perceptual priming of different stimuli such as faces and objects (Guillaume et al., 2009).

\subsubsection{Time-frequency analysis}

To investigate whether differences in different frequency bands indicate age related cognitive changes as was shown in PD patients and patients with $\mathrm{MCl}$ (Cummins et al., 2008; He, Zhang, Chen, Xie, Gan, Wang, et al., 2017; Latreille et al., 2016), we calculated the mean power of different frequency bands such as alpha, beta, gamma and theta. For the time frequency analysis, each $3000 \mathrm{~ms}$ epoch was transformed to the time-frequency domain using a fast Fourier Transform with the application of a Hanning taper and a sliding window of frequency-dependent size (5 cycles of the frequency of interest per window). Each of the time windows was shifted by $10 \mathrm{~ms}$ along the time domain. The mean power across trials for each frequency band (Theta: 4-7 Hz, Alpha: 8-13 Hz, Beta: $14-20 \mathrm{~Hz}$ and Low-Gamma: $25-40 \mathrm{~Hz}$ ) was calculated as percentage 


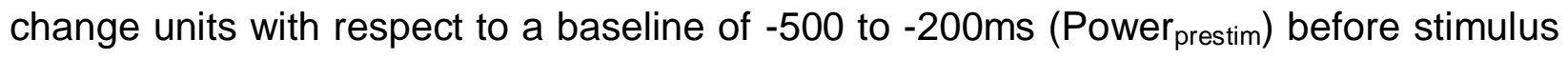
presentation. A single value of mean power change was obtained for each subject for

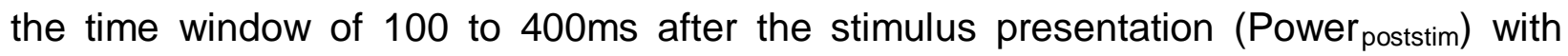
respect to the baseline $\left(\right.$ Power change $=\left(\right.$ Power $_{\text {poststim }}-$ Power $\left._{\text {prestim }}\right) /$ Power $_{\text {prestim }}$ *100). This value was used for analyzing differences between the groups and the correlation of band mean power with measures of cognitive and perceptual performance.

\subsubsection{Variability quenching}

To test age-related changes in neural variability we computed trial-by-trial variability separately for each subject computed for each time point in the epochs (-500 to $500 \mathrm{~ms}$ ) for the electrodes of interest. Trials were analyzed for each of the conditions separately (cars at $100 \%$ or $10 \%$ contrast, faces at $100 \%$ or $10 \%$ contrast or scrambled images at $100 \%$ or $10 \%$ contrast) and also grouped by contrast level. To calculate the variability quenching elicited by the presentation of the stimulus, two time windows were defined: the prestimulus time window (-200 to $0 \mathrm{~ms}$ ) and the post-stimulus time window (100 to $400 \mathrm{~ms})$. Then, absolute trial-by-trial variability for the pre and poststimulus intervals was computed as the mean variance of all the time points in the prestimulus ( $\left.\operatorname{Var}_{\text {pre }}\right)$ and poststimulus ( $\operatorname{Var}_{\text {pos }}$ ) time windows. Relative trial-by-trial variability (i.e. quenching level) was computed as the percentage of the difference between the variance in the poststimulus interval and the prestimulus interval based on the calculations performed by (Arazi, Gonen-Yaacovi, et al., 2017) as shown in the following equation:

$$
\text { Relative Variance }(\% \text { change })=\frac{\text { Var }_{\text {pos }}-\text { Var }_{\text {pre }}}{\text { Var }_{\text {pre }}} * 100
$$




\subsubsection{Heart Rate Variability}

One electrode of the electrodes of the 64 chanel system was placed on the back, thoracal paravertebral. The data were collected with a sampling rate of $1000 \mathrm{~Hz}$ resulting in a temporal resolution of $1 \mathrm{~ms}$ for the time interval between consecutive Rpeaks of the heart signal (RR interval). ECG was recorded during the 8 blocks of the task and a 2 minutes Resting state with eyes closed before and after the task. $\mathrm{R}$ peak detection was performed using Brain Vision Analyzer (Brain Products $\mathrm{GmbH}$, Gilching, Germany). To exclude artifacts RR intervals which differed more than $25 \%$ from previous and subsequent RR intervals (Malik, Cripps, Farrell, \& Camm, 1989). Excluded RR intervals were replaced by conventional spline interpolation providing the same data length or same number of beats (Luque-Casado et al., 2016). Further analyses were conducted using Kubios with smoothness prior mentod with a Labda value of 500 to remove disturbing low frequency baseline trend components (Tarvainen, Niskanen, Lipponen, Ranta-Aho, \& Karjalainen, 2014).

\subsubsection{Statistical Analyses}

\subsubsection{Neuropsychological tests}

General differences in demographic and neuropsychological factors such as age and depressive symptoms scored with BDI were tested using t-tests. Mann-Whitney test was used when the assumption of normality was violated for testing group differences in years of education, MMSE, Trail Making Test part A, part B, Verbal Fluency, Stroop Test, Simple Rection time Test, Finger Tapping Test, Mars Letters Contrast Sensitivity Test. To test group differences in gender and handedness we used Chi Square Test. 


\subsubsection{Task and Neural measures}

To test whether Healthy Elderly would show worse performance, higher behavioral variability (Bielak et al., 2010; Gorus et al., 2008; MacDonald et al., 2006), differences in neural variability and power of frequency bands, especially in theta power (Cozac et al., 2016) we performed a mixed repeated measures ANOVA with the whithin subject factors, contrast (two levels: 10\% and 100\%), category (three levels: face, car, scrambled) and group as a between subject factor with two levels, Healthy Young and Healthy Elderly. Using the above described ANOVA we tested group differences of the performance measures such as Proportion Correct Recognition (proportion correct categorization of faces, cars and scramble images), Perceptual Error Score (PES) consisting of category confusions and misses, mean recognition time, coefficient of recognition time variability (individual recongition time mean divided by individual standart deviation of recogniton time) (CVRT). We used the reteated measures ANOVA to investigated differences for the neural correlates of task performance such as P1, P3 amplitude, change in frequency power of alpha, beta, gamma and theta bands and variability quenching for the time windows, $100-400 \mathrm{~ms}, 100-200 \mathrm{~ms}, 200-300 \mathrm{~ms}$ and 300-400 ms after stimulus presentation. Greenhouse- Geisser corrected statistics (F and $p$ values) were reported. Post hoc test were t-tests and were Bonferroni corrected by dividing the signficance level of 0.05 by the amount of comparisons (CVRT for the three different categories $p<0.016$; ERP and frequency power for the six comparisons of the three categories and two contrasts $p<0.0083 ; p<$; variability quenching for the eight comparisons with four comparisons (three categories and one contrast pooled over all categories) within each group $p<0.00625)$. 


\subsubsection{Heart Rate Variability}

To investigate age differerences in cardiac variability we tested differences in HRV between Healthy Young and Healthy Elderly as well as in RMSSD (root mean square of successive differences), High frequency (HF) $(0.15$ to $0.40 \mathrm{~Hz})$, Low frequenchy (LF) (0.04 to $0.15 \mathrm{~Hz}$ ) and LF to HF ratio, in the Resting state before the task, during task performance and Resting state after the task using t-tests. Bonferroni correction was applied for multiple comparisons of different heart rate variability measures, resulting in a significance threshold of $p<0.0042$.

\subsubsection{Correlations}

To test whether intra-individual variability is related to cognitive changes, neural variability, change in power of freuquency bands and cardiac variability we performed Spearman's rho correlations separate for each group between task measurements such as intra-individual variability and perceptual error score, cognitive measurements such as Trail Making Test and Stroop Test, neural measurements such as variability quenching, change in frequency power, P1 and P3 amplite, HRV measurements such as RMSSD, HF power, LF power and LF to HF ratio. Outlier were defined as $2 \mathrm{SD}$ below or above the mean within the group were excluded and spearman's rank correlations were recalculated to test their stability. 


\subsection{Results}

To test whether there are general cognitive differences in Healthy Young and Healthy Elderly we tested differences on cognitve tests such as the Trail Making Test, Stroop test expecting Healthy Elderly to need more time to solve the tests. Healthy Young needed less time to solve the Trail Making Test A and B (Trail Making Test A: $U=238$, $p=0.0003$, Trail Making Test $B: U=160, p=0.02$, Stroop test congurent trials: $t(43)=-$ 5.77, $p<0.001$; Stroop test incongruent trials: $U(43)=48, p<0.001$ ) indicating lower cognitive function in Healthy Elderly (Table 3.1).

\subsubsection{Correct image recognition in the main task}

To test whether our task is sensitive in indicating minor cognitive changes we compared error proportions in Healthy Young and Healthy Elderly expecting Healthy Elderly to make more mistakes. Young and elderly subjects showed comparable proportion of recognition errors. Both groups made more errors in the lower contrast condition for faces, cars and scrambled images as illustrated in Figure 3.2 and Table 3.2. 
Table 3.2. Repeated measures mixed ANOVA with the factors contrast, category and group of Healthy Young $(\mathrm{N}=22)$ and Healthy Elderly $(\mathrm{N}=24)$

\begin{tabular}{|c|c|c|c|c|c|}
\hline & $\begin{array}{l}\text { Proportion } \\
\text { Correct } \\
\text { Recognition }\end{array}$ & $\begin{array}{l}\text { Proportion } \\
\text { Misses }\end{array}$ & $\begin{array}{l}\text { Perceptual } \\
\text { Error Score }\end{array}$ & RT & CVRT \\
\hline & $F(p)$ & $F(p)$ & $F(p)$ & $F(p)$ & $F(p)$ \\
\hline Contrast & $\begin{array}{l}501.44 \\
(<0.0001)^{\star *}\end{array}$ & $\begin{array}{l}27.23 \\
(<0.0001)^{\star *}\end{array}$ & $\begin{array}{l}190.19 \\
(<0.0001)^{\star *}\end{array}$ & $\begin{array}{l}283.60 \\
(<0.0001)^{\star *}\end{array}$ & $\begin{array}{l}16.88 \\
(<0.0001)^{\star *}\end{array}$ \\
\hline Category & $\begin{array}{l}5.89 \\
(=0.005)^{\star *}\end{array}$ & $3.65(=0.04)^{*}$ & & $1.07(=0.35)$ & $0.78(=0.46)$ \\
\hline Group & $1.92(=0.17)$ & $1.19(=0.28)$ & $5.78(=0.02)^{*}$ & $3.04(=0.09)$ & $2.64(=0.11)$ \\
\hline $\begin{array}{l}\text { Contrast x } \\
\text { Group }\end{array}$ & $0.07(=0.79)$ & $3.94(=0.054)$ & $1.04(=0.13)$ & $8.12(=0.007)^{\star}$ & $0.27(=0.60)$ \\
\hline $\begin{array}{l}\text { Category x } \\
\text { Group }\end{array}$ & $0.67(=0.50)$ & $0.38(=0.67)$ & & $0.04(=0.95)$ & $3.88(=0.03)^{\star}$ \\
\hline $\begin{array}{l}\text { Contrast x } \\
\text { Category x } \\
\text { Group }\end{array}$ & $0.37(=0.6)$ & $0.38(=0.67)$ & & $1.32(=0.27)$ & $0.05(=0.94)$ \\
\hline $\begin{array}{l}\text { Contrast x } \\
\text { Category }\end{array}$ & $\begin{array}{l}10.55 \\
(=0.0001)^{\star *}\end{array}$ & $3.65(=0.04)^{\star}$ & & $0.59(=0.52)$ & $1.06(=0.36)$ \\
\hline $\begin{array}{l}\mathrm{RT} \text {, Recognit } \\
\text { recognition tir } \\
\text { Between-sub } \\
\text { face, car, Co } \\
{ }^{*} p<0.05 ;{ }^{* *}\end{array}$ & $\begin{array}{l}\text { mes; CVRT, } \\
\text { factor: Group } \\
: 2 \text { levels: cor } \\
005\end{array}$ & $\begin{array}{l}\text { n individual ve } \\
\text { _evels: Young } \\
\text { st } 10 \% \text {, contra }\end{array}$ & $\begin{array}{l}\text { ility coefficien } \\
\text { lerly Within-s } \\
00 \%\end{array}$ & $\begin{array}{l}\text { dividual SD/ir } \\
\text { cts factors: C }\end{array}$ & $\begin{array}{l}\text { dual mean) of } \\
\text { ory: } 3 \text { levels: }\end{array}$ \\
\hline
\end{tabular}


A

$\star *$
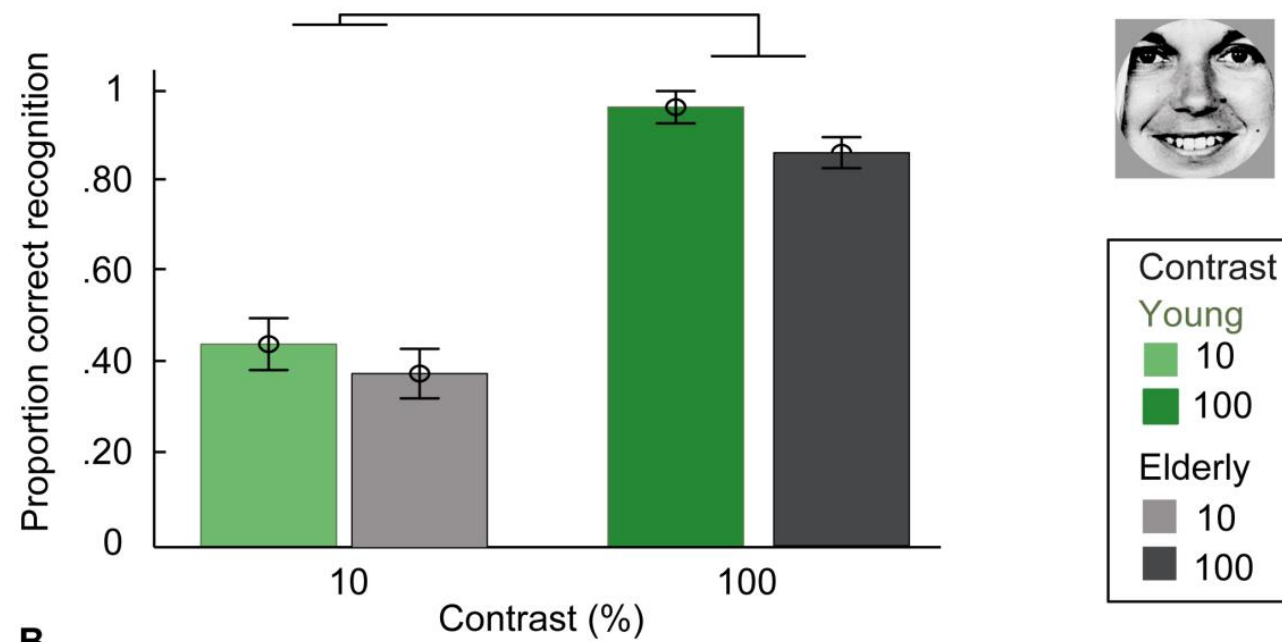

Contrast (\%)

Young

10

100

Elderly

10

100

B

* *
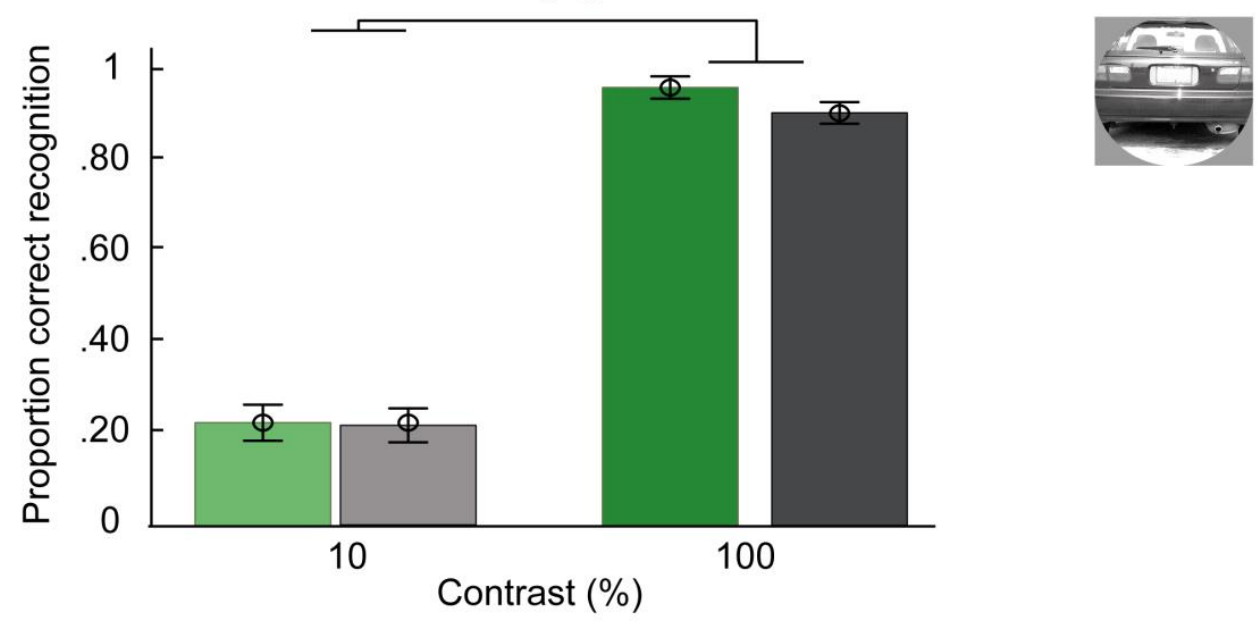

C
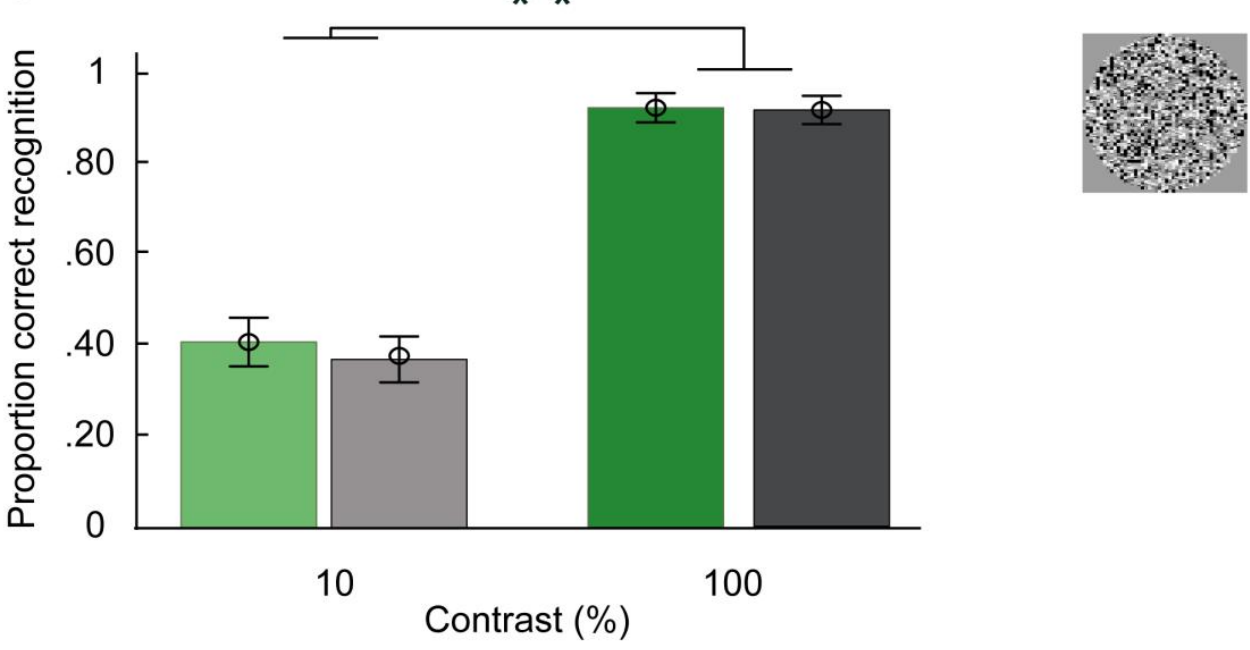

Figure 3.2. Proportion correct image recognition. Mean proportion of correct recognition of A) faces,

B) cars and C) scrambled images. Means across subjects in each group are shown. Error bars denote S.E.M. across subjects. ${ }^{* *}$ denotes a significant difference for low and high contrast condition, $p<0.005$ (black star: 10\% Contrast vs. 100\% Contrast as assessed by the ANOVA). 
Figure 3.3 shows more missed responses, within the 2 seconds of stimulus presentation in the low contrast condition in Healthy Elderly and Healthy Young volunteers (Table 3.2).

A
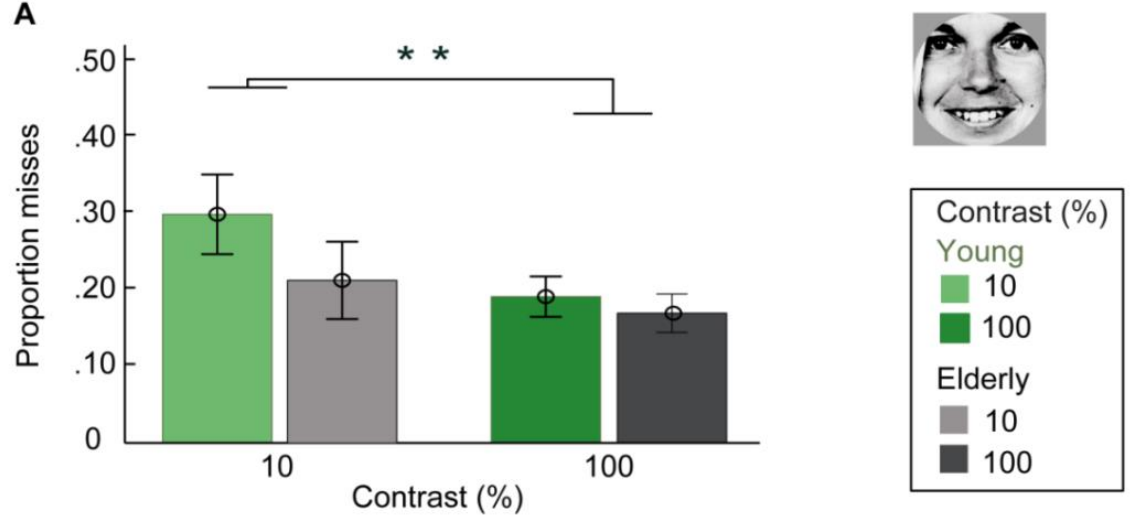

B
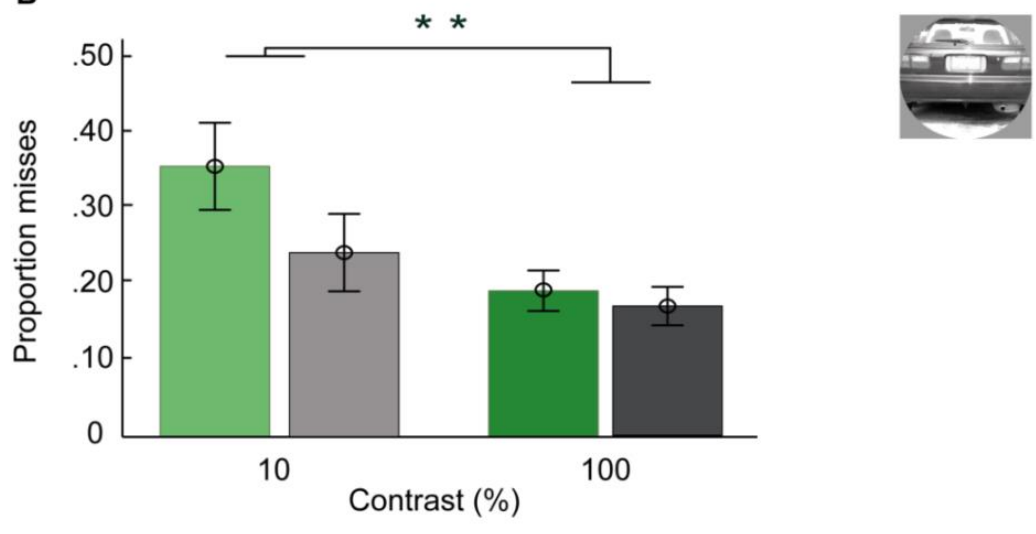

C
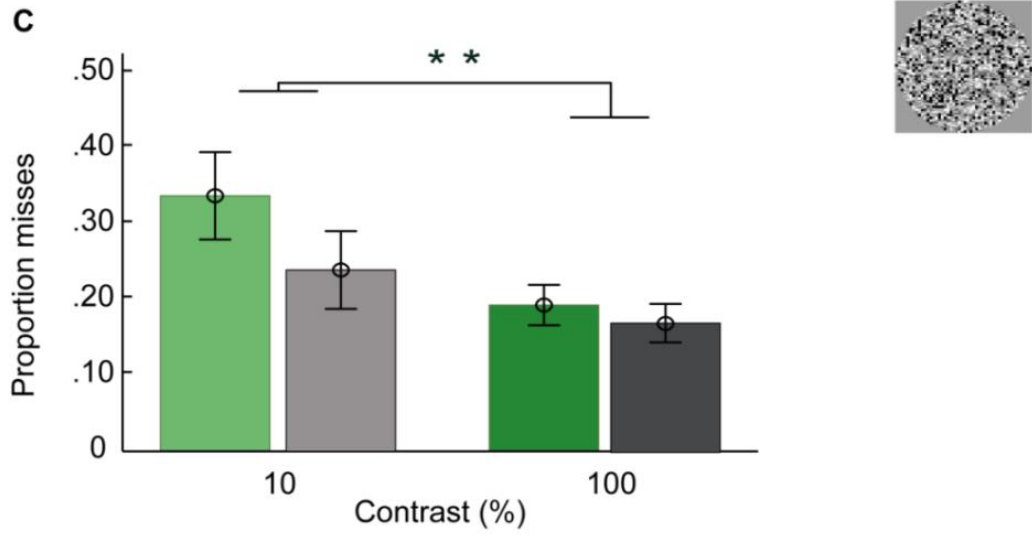

Figure 3.3. Proportion misses. Mean proportion of correct recognition of A) faces, B) cars and C) scrambled images. Means across subjects in each group are shown. Error bars denote S.E.M. across subjects. ${ }^{* *}$ denotes a significant difference, $p<0.005$ (black star: $10 \%$ Contrast vs. $100 \%$ Contrast as assessed by the ANOVA). 


\subsubsection{Perceptual Error Score (PES)}

Assuming that a combined sum score (Shine et al., 2012) of different image recognition errors is more robust as was shown in clinical studies than the separate ones, we computed a sum error score (PES) of category confusion, misses and false real image detection in scrambled images, similar to Shine et al. (Shine et al., 2012). Both, Healthy Young and Elderly show an increase in perceptual errors for the low contrast condition (Figure 3.4A). Healthy Elderly showed an increase in perceptual errors compared to Healthy Young (Healthy Young: 0.35 , Healthy Elderly: $0.43, F(1,44)=5.78, p=0.02$ (Figure 3.4B, Table 3.2). 
A

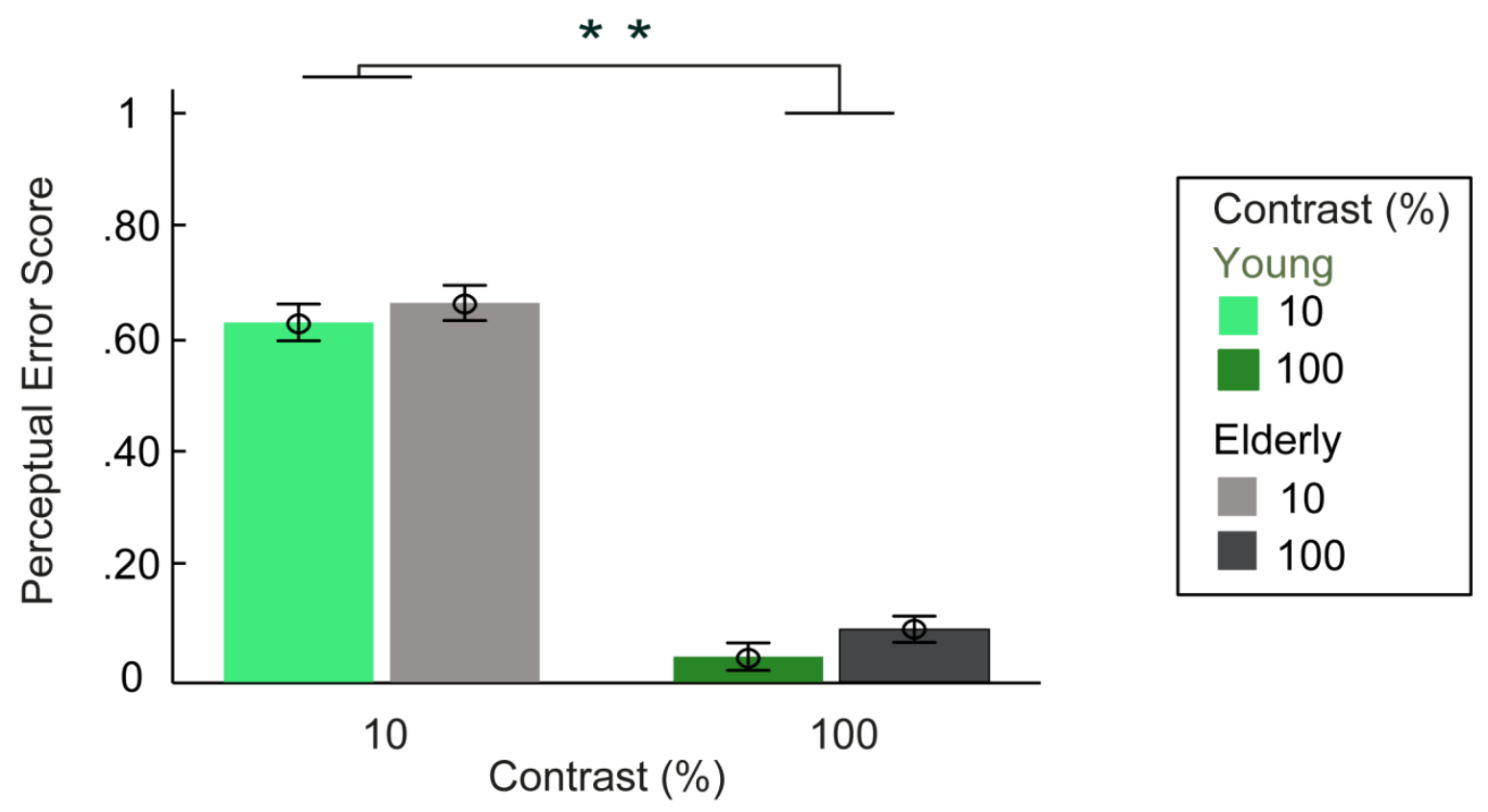

B

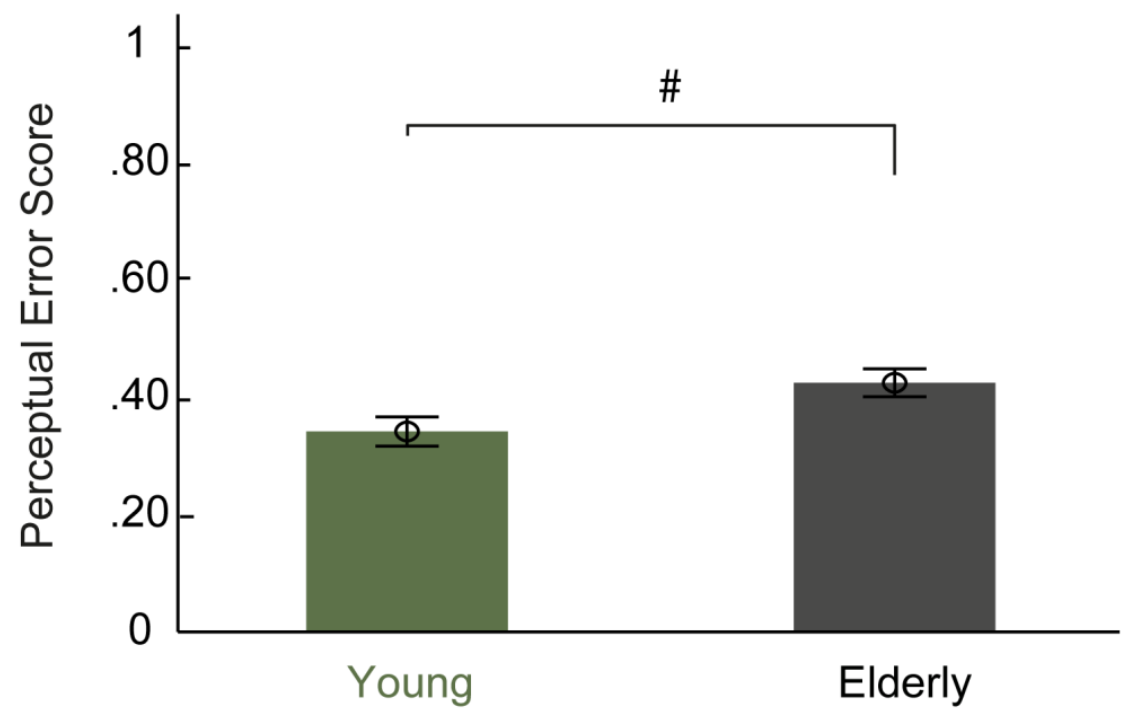

Figure 3.4. Perceptual Error Score. Mean proportion Perceptual Error Score, separated by subject group, Healthy Young $(\mathrm{N}=22)$ and Healthy Elderly $(\mathrm{N}=24)$. In all panels, means across subjects in each group are shown. Error bars denote S.E.M. across subjects for A) differences between groups in low and high contrast and B) mean difference between groups independent of contrast level. ${ }^{* *}$ denotes a significant difference, $p<0.005$ (black star as assessed by the ANOVA) \# denotes a difference $p<0.05$ $(p<0.01$ Bonferroni corrected threshold). 


\subsubsection{Mean Recognition times}

To investigate whether age has effects on response speed we compared both groups expecting Healthy Elderly to be slower in responding (Deary \& Der, 2005). In contrast to

our expectation Healthy Elderly volunteers responded faster in categorization of the stimuli in the low contrast condition $(t(44)=2.48, p=0.02)$ (Figure 3.5, Table 3.2). 

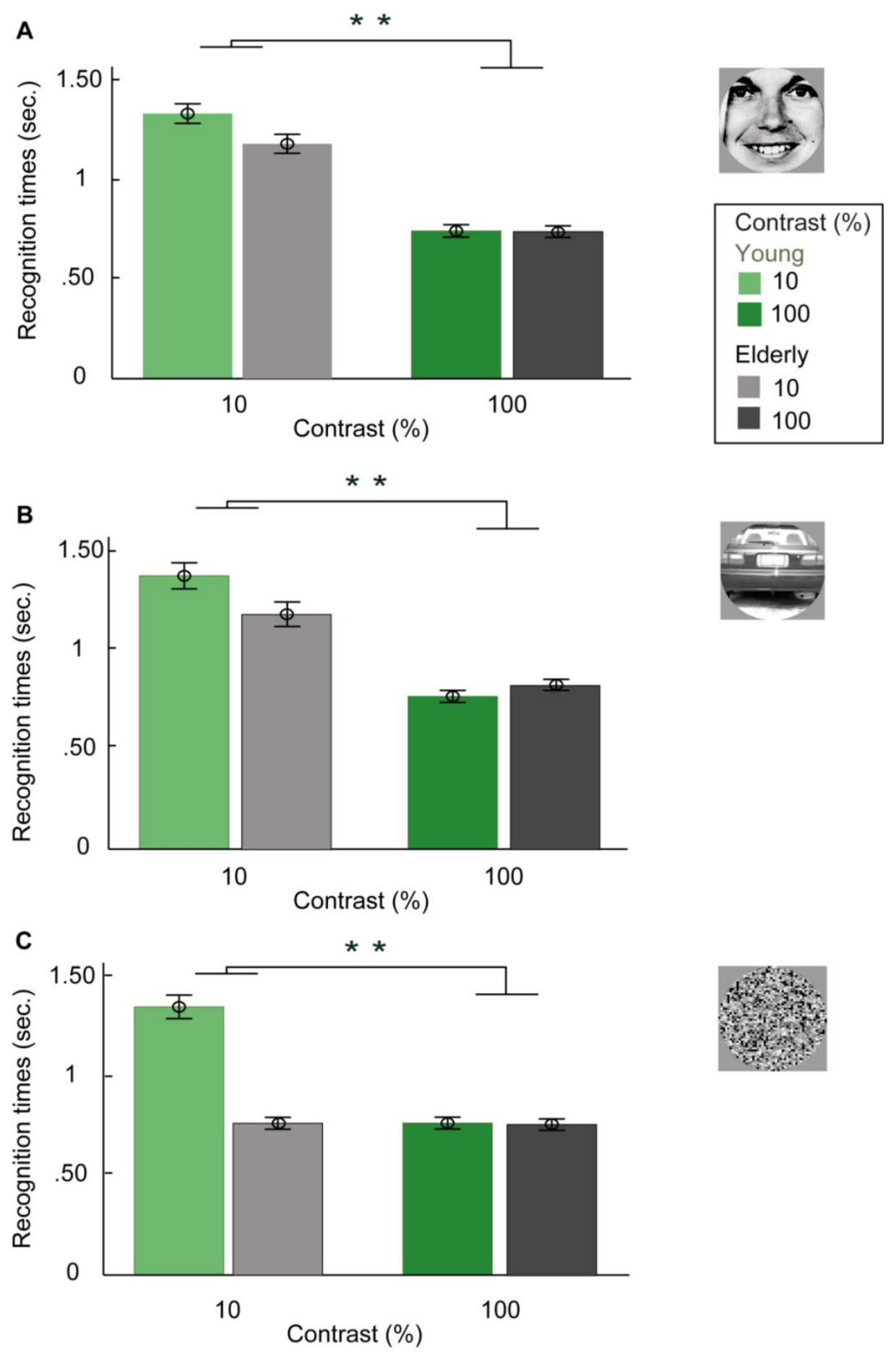

Figure 3.5. Mean Recognition times. Mean Recognition time of A) faces, B) cars and C) scrambled images, separated by subject group, Healthy Young $(\mathrm{N}=22)$ and Healthy Elderly $(\mathrm{N}=24)$. In all panels, means across subjects in each group are shown. Error bars denote S.E.M. across subjects. ${ }^{* *}$ denotes a significant difference between low and high Contrast condition, $p<0.005$ (black star as assessed by the ANOVA). 
Figure 3.6 illustrates that in Healthy Young volunteers higher recognition times are related to lower proportion of correct categorization for facial and scrambled images showing that those Healthy Young volunteers who made more mistakes needed more time which does not provide an explanation for longer RT in Healthy Young.

A
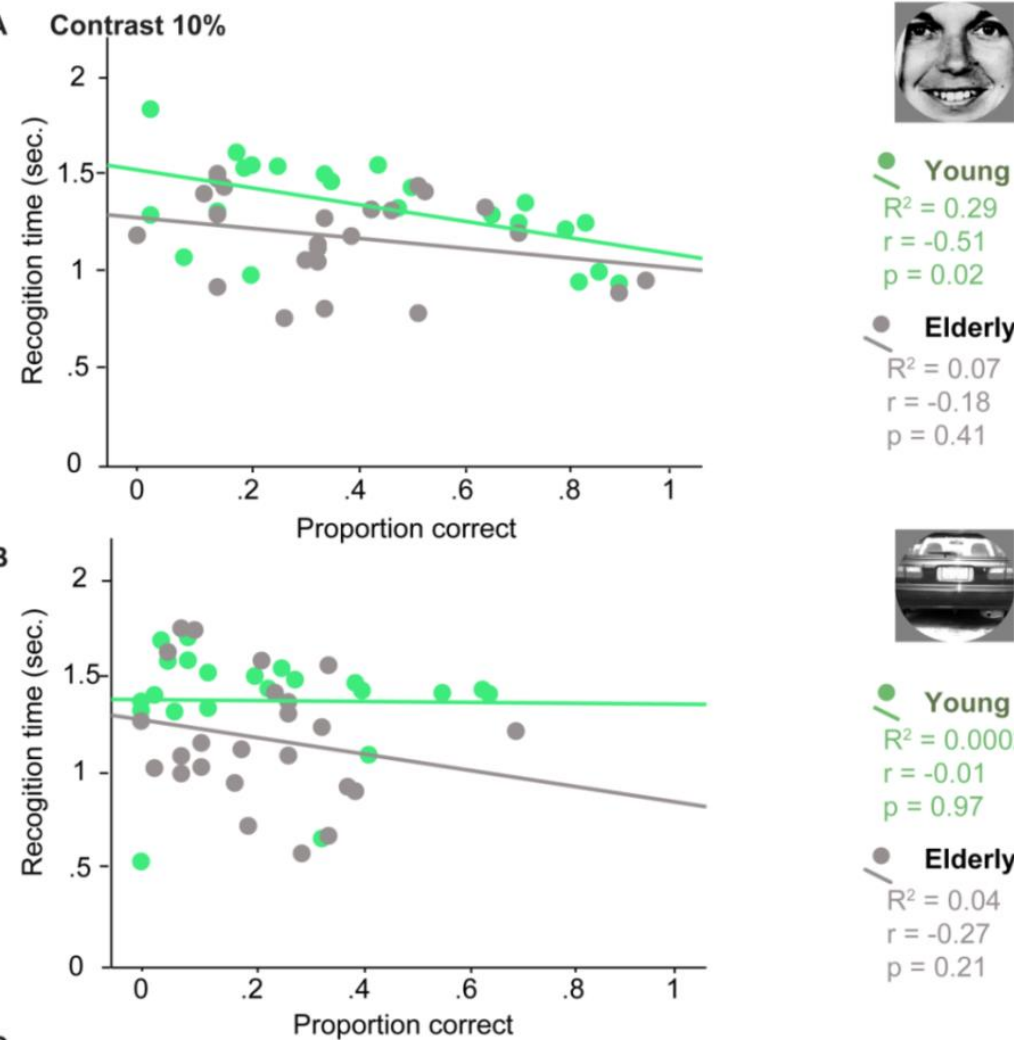

C

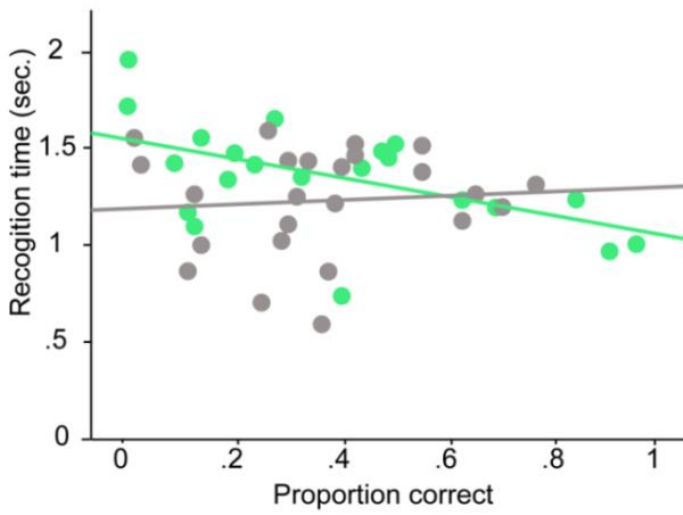

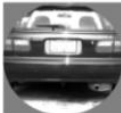

- Young

$R^{2}=0.29$

$r=-0.51$

$p=0.02$

- Elderly

$R^{2}=0.07$

$r=-0.18$

$p=0.41$
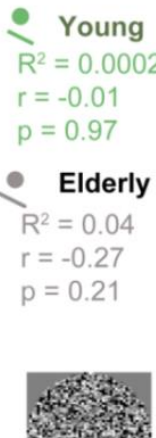

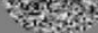

- Young

$\mathrm{R}^{2}=0.29$

$r=-0.48$

$p=0.02$

- Elderly

$R^{2}=0.007$

$r=-0.09$

$p=0.69$

Figure 3.6. Correlation of Recognition time and Proportion correct performance. Correlation of the low contrast condition for proportion correct displayed on the $\mathrm{x}$-axis and recognition times on the $y$-axis for A) facial images B) car images C) scrambled images. Green dots show the individual values of Healthy Young volunteers $(N=22)$ and grey dots the individual values of Healthy Elderly volunteers $(N=24)$. 


\subsubsection{Intra-individual variability of recognition times}

The previous study of our group showed that CVRT was increased in hallucinating PD patients. Investigating possible mechanisms of CVRT we could show that both, Young and Elderly show increased CVRT for the high contrast condition of car and scrambled stimuli (Figure 3.7 B and C, Table 3.2). In addition, the "Group x Object" interaction indicates differences between the Healthy Young and Healthy Elderly in CVRT for certain stimuli $(F(1,44)=3.88, p=0.03)$. Elderly volunteers show increased variability for cars compared to Healthy Young subjects (Young: 0.16, Elderly: $0.20, \mathrm{t}(44)=-2.94$, $\mathrm{p}=0.005)$ (Figure 3.7 D). 
A
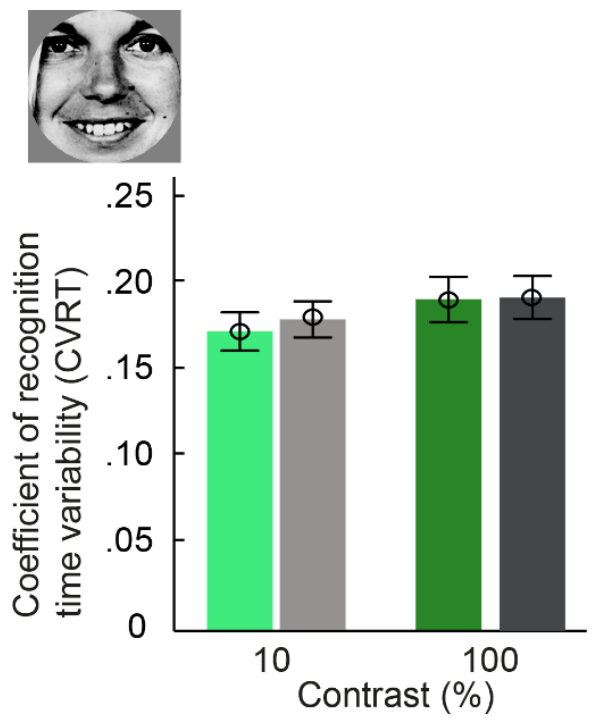

B
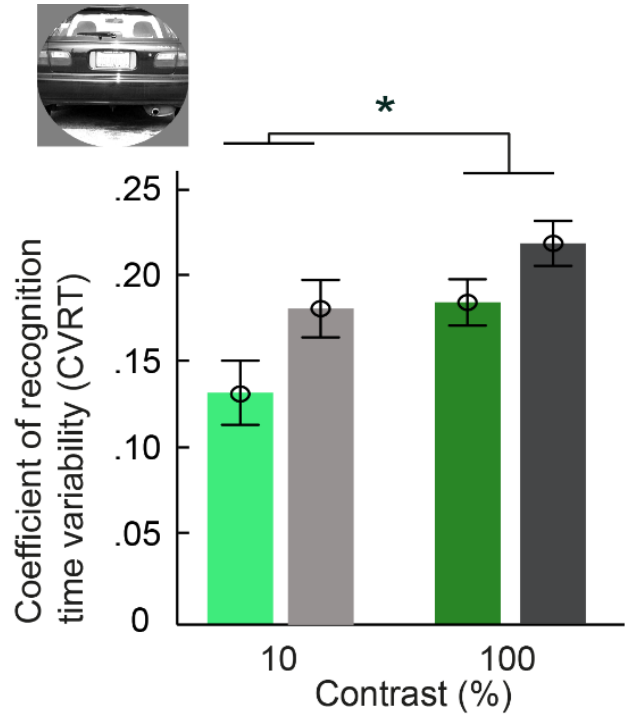

\begin{tabular}{|ccccc|}
\hline & \multicolumn{2}{c|}{ Contrast (\%) } & & Contrast (\%) \\
Young & 10 & 100 & Elderly & $10 \square$ \\
\hline
\end{tabular}

C

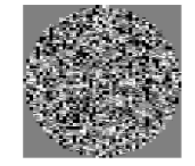

을

25
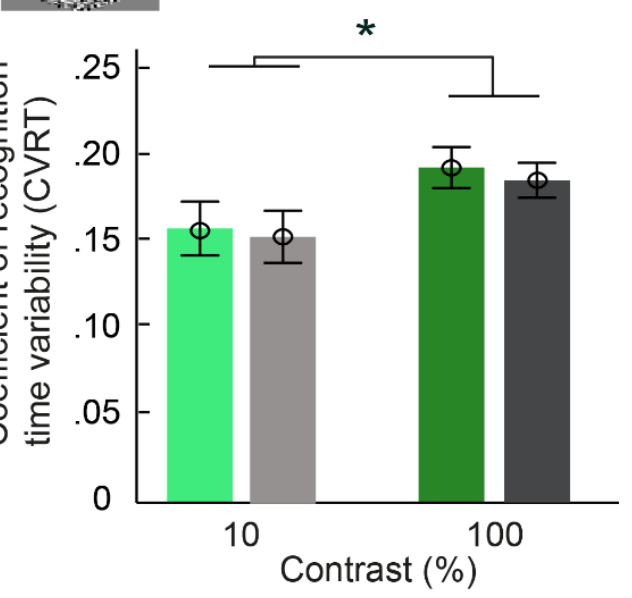

D
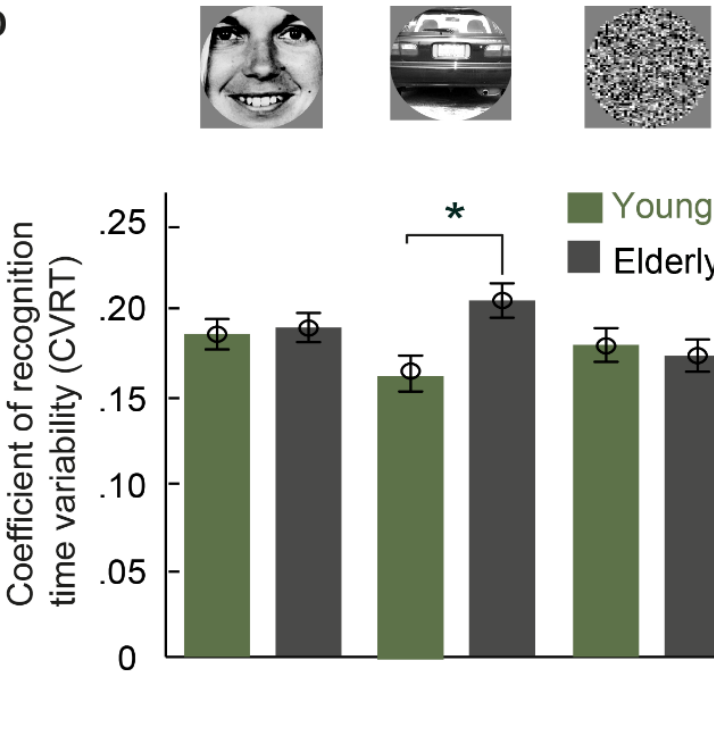

Young

Elderly $\Phi$
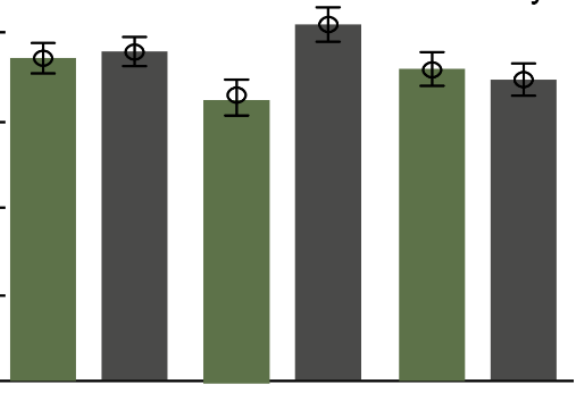

Figure 3.7. Differences in Coefficient of recognition times variability. Average of individual variability in RT (CVRT = individual standard deviation of RT divided by the individual RT mean), separated by stimulus type, A) face, B) car, C) scrambled image, contrast level 10\%, 100\% and by subject group, Healthy Young $(N=22)$ in green and Healthy Elderly $(N=24)$ in gray. In $D)$ averages of CVRT separated by stimulus type and subject group are shown. In all panels, means across subjects in each group are shown. Error bars denote S.E.M. across subjects. * denotes a significant difference between high and low contrast condition, $\mathrm{p}<0.05$ (black star as assessed by post-hoc tests). 


\subsubsection{Correlation of intra-individual variability of recognition times (CVRT) and cognitive measures}

To investigate factors that might explain increase in behavioral intra-individual variability of recognition times in Healthy Young and Healthy Elderly we performed Spearman's rank correlations between CVRT and cognitive measures such as the time of the Trail Making Test and Verbal Fluency Test. Figure 3.8, 3.9 illustrate that only in Healthy Elderly increased CVRT for low contrast faces was related to longer times to complete Trail Making Test part $A\left(r_{s}=0.49, p=0.02\right)$ and average of all low contrast stimuli to longer times to complete the Trail Making Test part A and part $B(p \leq 0.03)$, indicating that reduced executive functioning is related to increased behavioral variability in Healthy Elderly volunteers. These correlations remain significant after removing six outliers which scored 2 SD below or above the mean within the group (Figure 3.8, 3.9). 
A

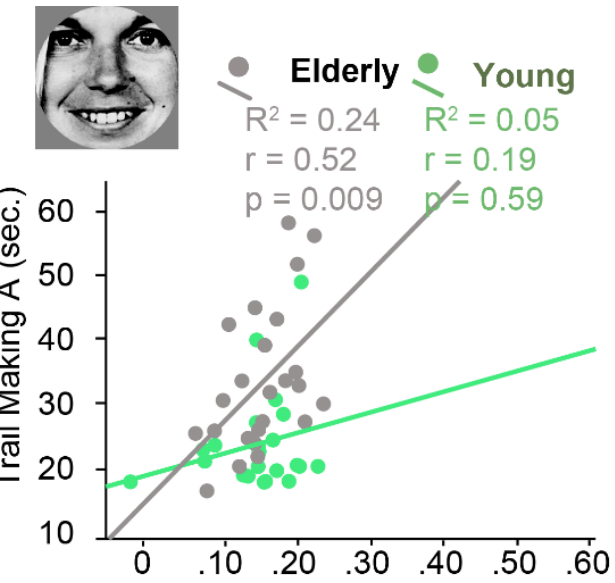

\section{Without outlier}

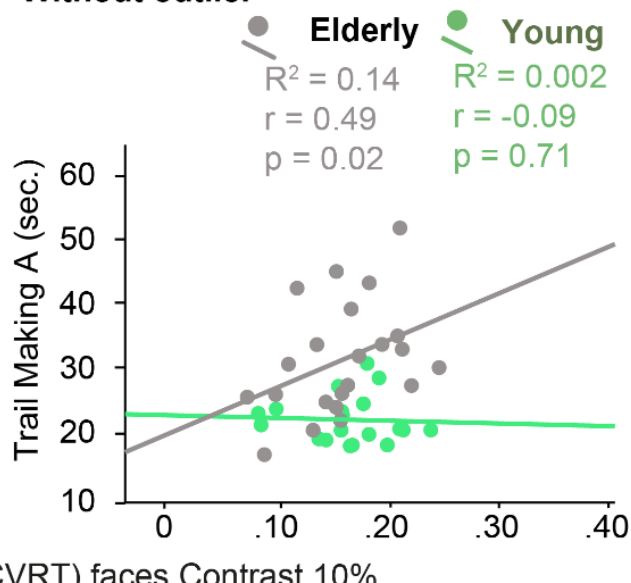

B
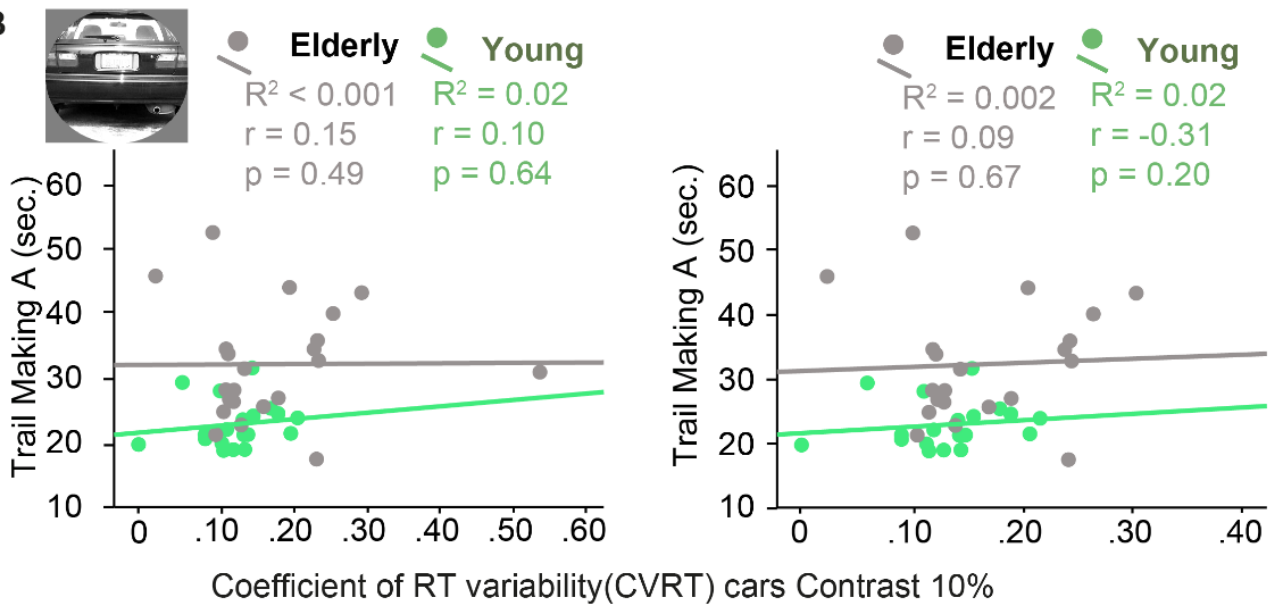

C

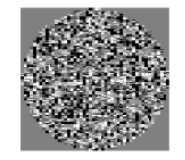

Coefficient of RT variability(CVRT) cars Contrast $10 \%$
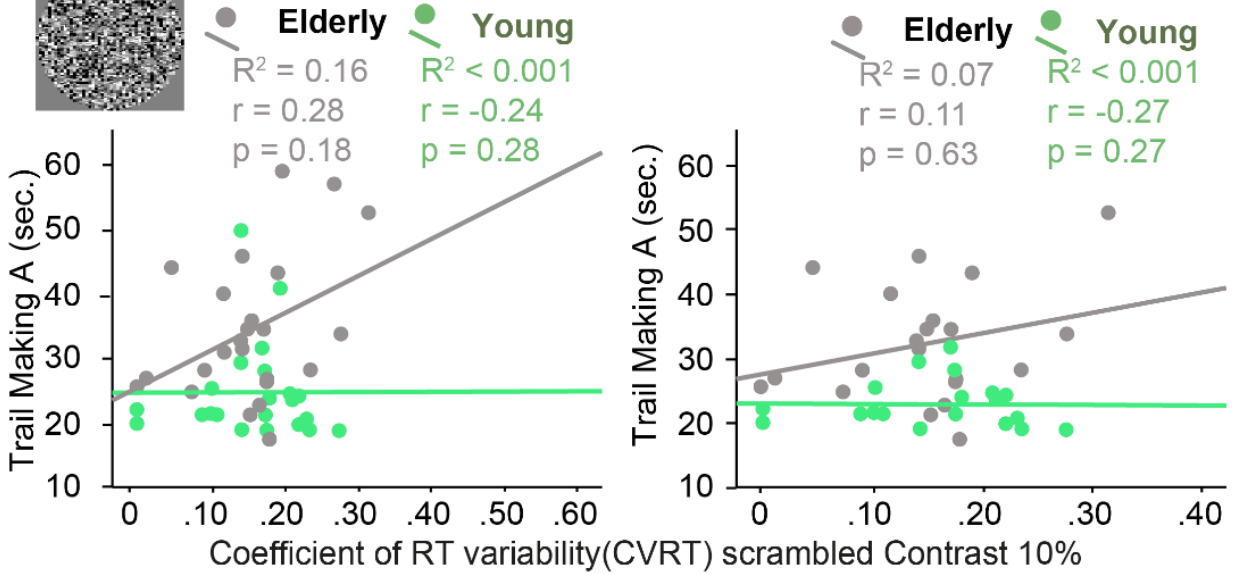

Figure 3.8. Association of coefficient of recognition time variability with Trail Making Test part A. Correlation between CVRT for A) faces B) cars and C) scrambled images Contrast $10 \%$ displayed on the $\mathrm{x}$-axis and time solving Trail Making Test part A on the y-axis, left whole sample and right without outlier ( $\pm 2 \mathrm{SD}$ from the average within the group). Green dots show the individual values of Healthy Young volunteers $(N=22$, without outliers: $N=19)$ and grey dots the individual values of Healthy Elderly volunteers $(N=24$, without outliers: $N=21)$. 
A

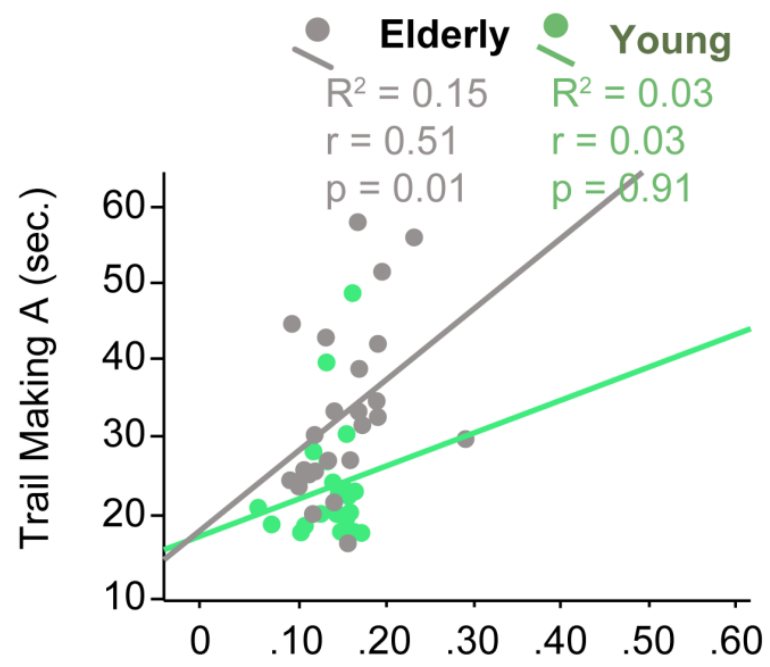

Without outlier

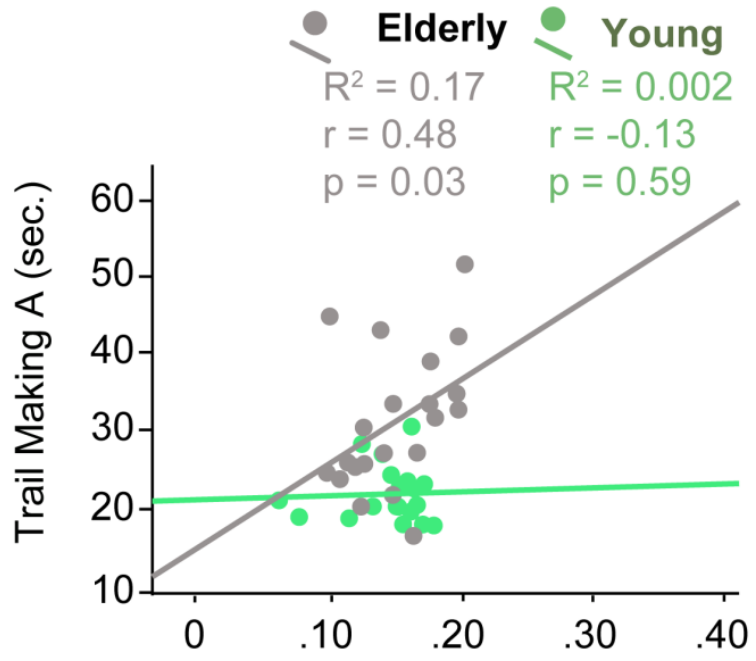

Coefficient of RT variability(CVRT) Contrast $10 \%$

в
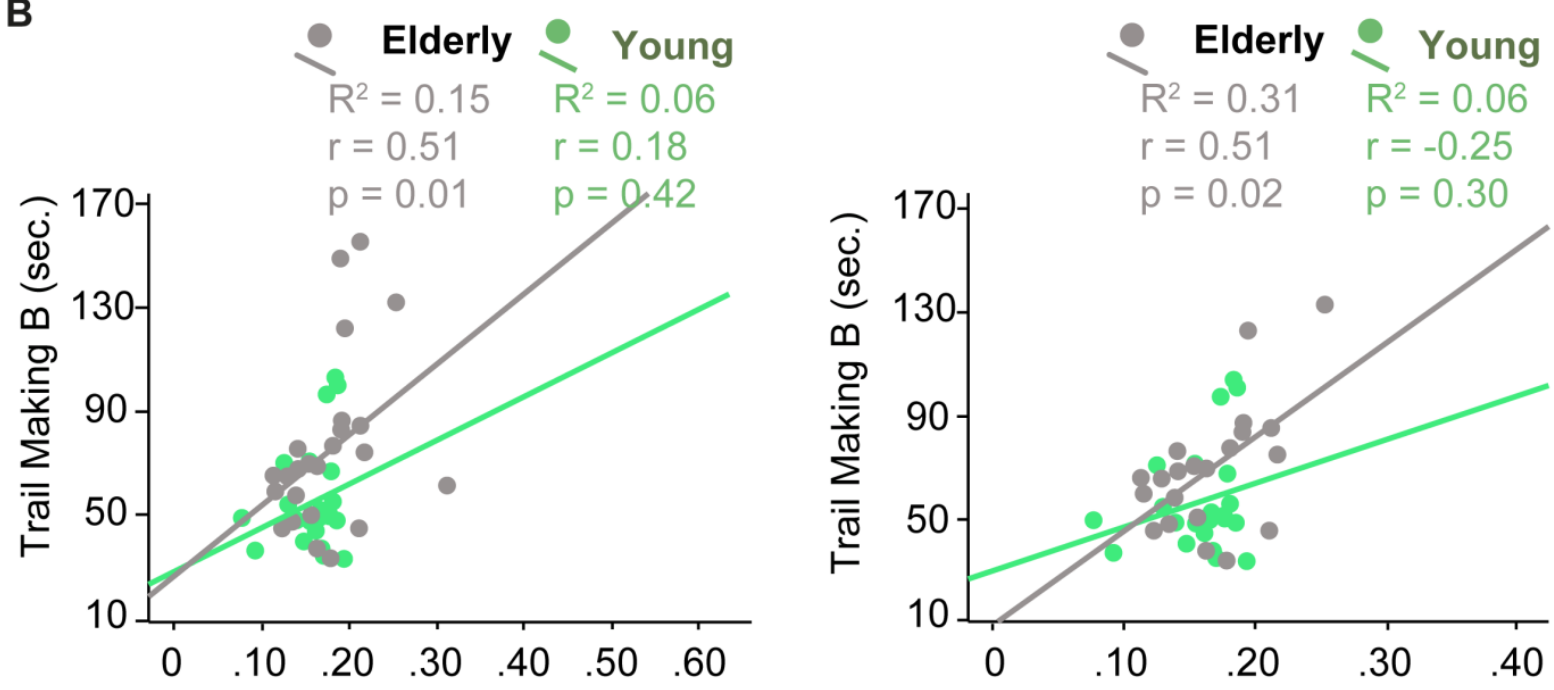

Coefficient of RT variability(CVRT) Contrast $10 \%$

Figure 3.9. Association of coefficient of recognition time variability with Trail Making Test part A and B. Correlation between CVRT average of all category types of Contrast $10 \%$ displayed on the $\mathrm{x}$-axis and time solving Trail Making Test A) part A and B) part B on the y-axis, left whole sample and right without outlier ( \pm 2 SD from the average within the group). Green dots show the individual values of Healthy Young volunteers $(\mathrm{N}=22$, without outliers: $N=19)$ and grey dots the individual values of Healthy Elderly volunteers $(\mathrm{N}=24$, without outliers: $N=21$ ). 


\subsubsection{Event-related Potentials}

To investigate differences in neural correlates of perceptual and cognitive processes we measured differences in P1 and P3 amplitude between Healthy Elderly and Healthy Young expecting lower amplitudes in Healthy Elderly (Hsieh \& Lin, 2017). The expected lower P1 Amplitude in Healthy Elderly is supported by the main effect of Group ( $F(1,44)$ $=5.57, p=0.023)$. Figure 3.10 illustrates the lower P1 Amplitude for low contrast condition and Figure 3.11 the lower P3 Amplitude for high contrast condition in Healthy Elderly compared to Healthy Young (Table 3.3). This is supported by the interaction effect of "Contrast $x$ Category $x$ Group" of $P 1(F(1,44)=5.22, p=0.01)$ and the interaction effect of "Contrast x Group" of P3 $(F(1,44)=18.59, p<0.0001)$. Post-hoc tests, Bonferroni corrected for multiple comparisons of the different conditions (significance threshold $p<0.0083$ ) showed lower P1 Amplitude for cars and scrambled images and a tendency for facial images in the low contrast condition and a tendency for cars in the high contrast condition in Healthy Elderly volunteers (Contrast 10\%: faces: $\mathrm{t}(44)=2.36, \mathrm{p}=0.02$; cars: $\mathrm{t}(44)=3.13, \mathrm{p}=0.003$; scrambled: $\mathrm{t}(44)=3.01, \mathrm{p}=$ 0.004; Contrast 100\%: faces: $\mathrm{t}(44)=1.78, \mathrm{p}=0.08$; cars: $\mathrm{t}(44)=2.74, \mathrm{p}=0.009$; scrambled: $\mathrm{t}(44)=0.03, p=0.98)$ and lower P3 amplitude for cars and a tendency for faces and scrambled images in the high contrast condition in Healthy Elderly compared to Healthy Young (Contrast 10\%: faces: $\mathrm{t}(44)=0.71, \mathrm{p}=0.48$; cars: $\mathrm{t}(44)=0.90, \mathrm{p}=$ 0.37 ; scrambled: $\mathrm{t}(44)=1.02, \mathrm{p}=0.32$; Contrast $100 \%$ : faces: $\mathrm{t}(44)=2.41, \mathrm{p}=0.02$; cars: $\mathrm{t}(44)=2.81, \mathrm{p}=0.007 ;$ scrambled: $\mathrm{t}(44)=2.27, \mathrm{p}=0.03$ ). 
Table 3.3. Event Related Potentials: Repeated measures mixed ANOVA with the factors contrast, category and group of Healthy Young $(\mathrm{N}=22)$ and Healthy Elderly $(\mathrm{N}=24)$

P1 Amplitude

$F(p)$

Contrast

Category

Group

Contrast x Group

Category x Group

Contrast x Category x Group

Contrast x Category

$4.19(=0.047)^{\star} \quad 81.69(<0.0001)^{\star *}$

$19.87(<0.0001)^{\star \star}$

$5.57(=0.023)^{\star}$

$1.16(=0.29)$

$5.37(=0.008)^{*}$

$5.22(=0.01)^{*}$

$16.36(<0.0001)^{\star \star}$

\section{P3 Amplitude}

$F(p)$

Between-subjects factor: Group: 2 Levels: Young, Elderly

Within-subjects factors: Category: 3 levels: face, car, Contrast: 2 levels: contrast 10\%, contrast 100\%

${ }^{*} p<0.05 ;{ }^{* *} p<0.005$ 

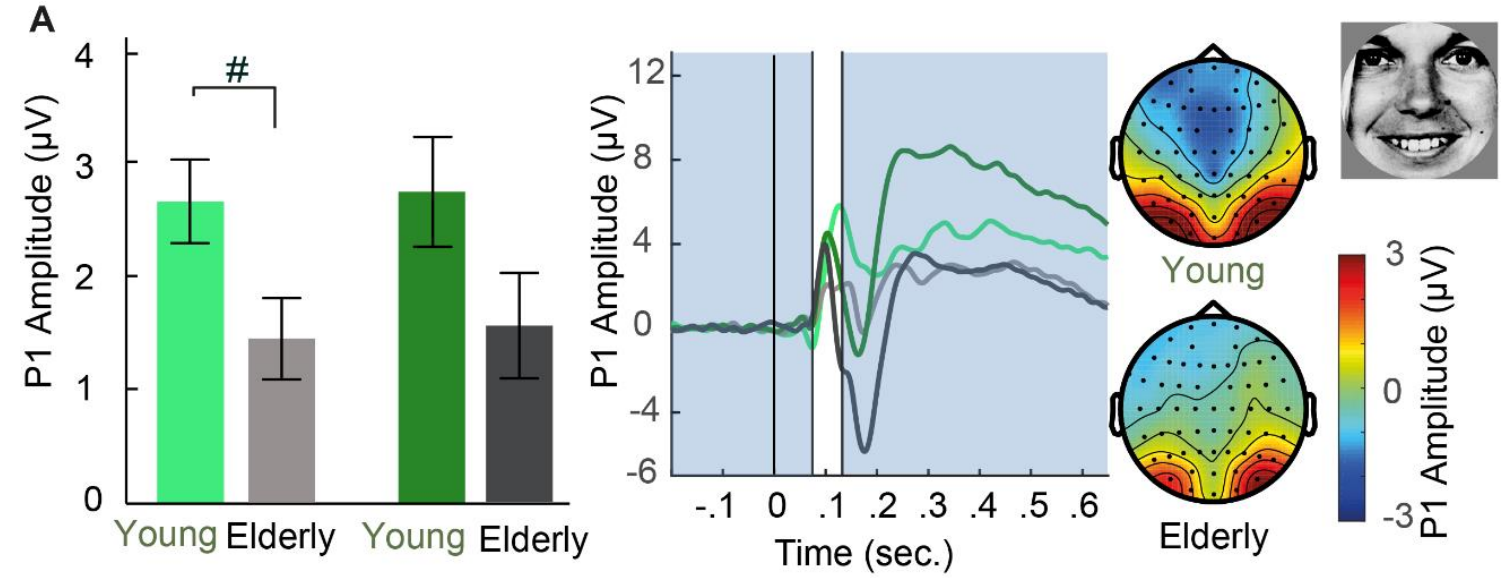

\begin{tabular}{|c|c|c|c|}
\hline & Contrast (\%) & & Contrast (\%) \\
\hline Young & $10 \square 100$ & Elderly & $10 \square 100$ \\
\hline
\end{tabular}
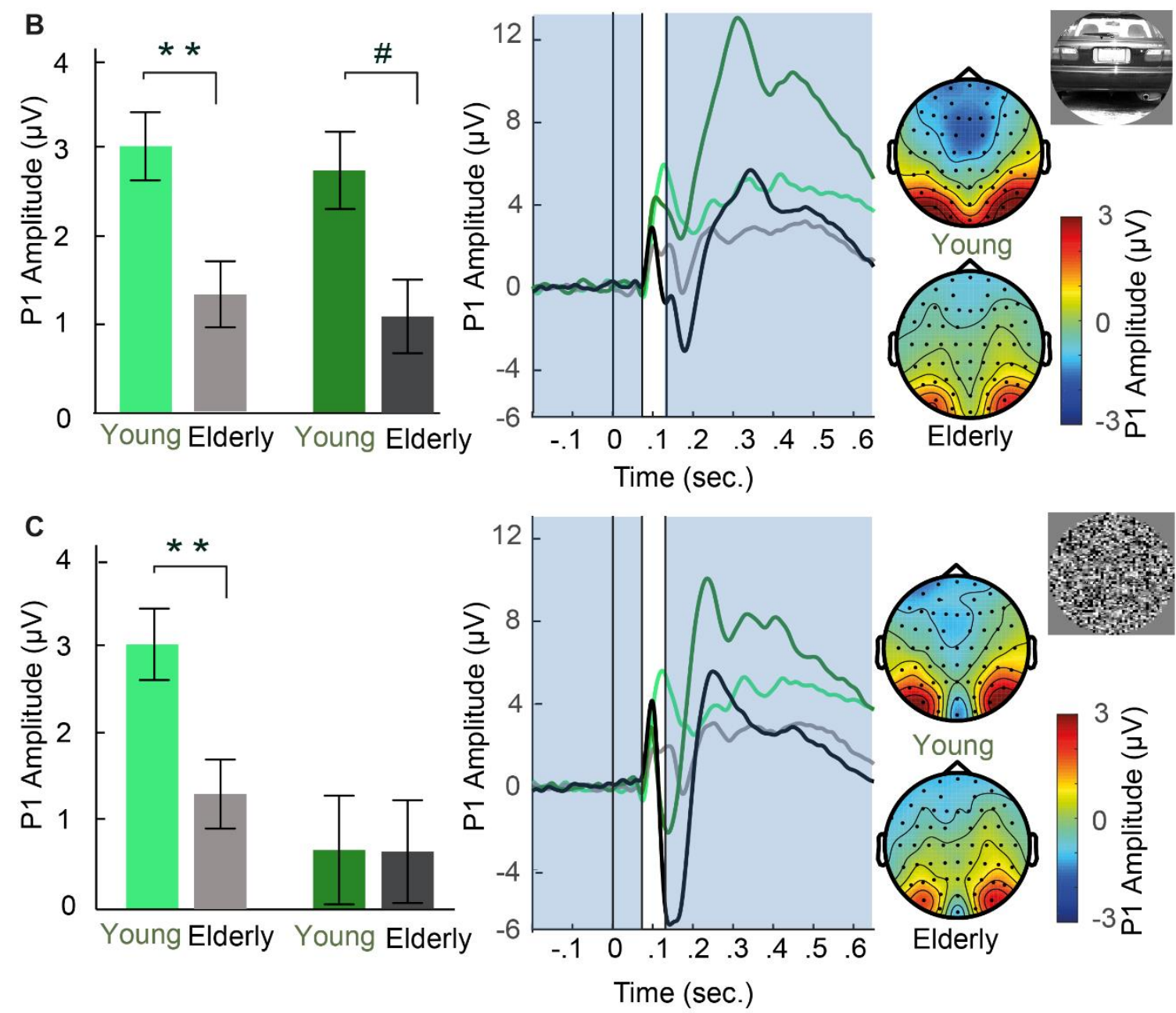

Figure 3.10: Differences in P1 Amplitude. P1 Amplitude derived from the electrodes O1, O2, separated by stimulus type, A) face, B) car, C) scrambled image and by subject group, Healthy Young $(\mathrm{N}=22)$ in green and Healthy Elderly $(\mathrm{N}=24)$ in gray. On the left in 
all panels, means of the P1 amplitude across subjects in each group are shown. Error bars denote S.E.M. across subjects. In the center P1 amplitude and on the right the spatial distribution of P1 Amplitude for the high contrast condition is shown. ** denotes a significant difference between Healthy Young and Healthy Elderly, $p<0.005$. ${ }^{*}$ denotes a significant difference between Healthy Young and Healthy Elderly, $p<0.008$ (black star as assessed by ANOVA and post-hoc t-tests). \# denotes significant difference without Bonferroni correction, $\mathrm{p}<0.05$ (assessed by ANOVA and post-hoc t-tests). On the right mean EEG activity is shown in green for Healthy Young $(N=22)$ and in grey for Healthy Elderly $(\mathrm{N}=24)$. 

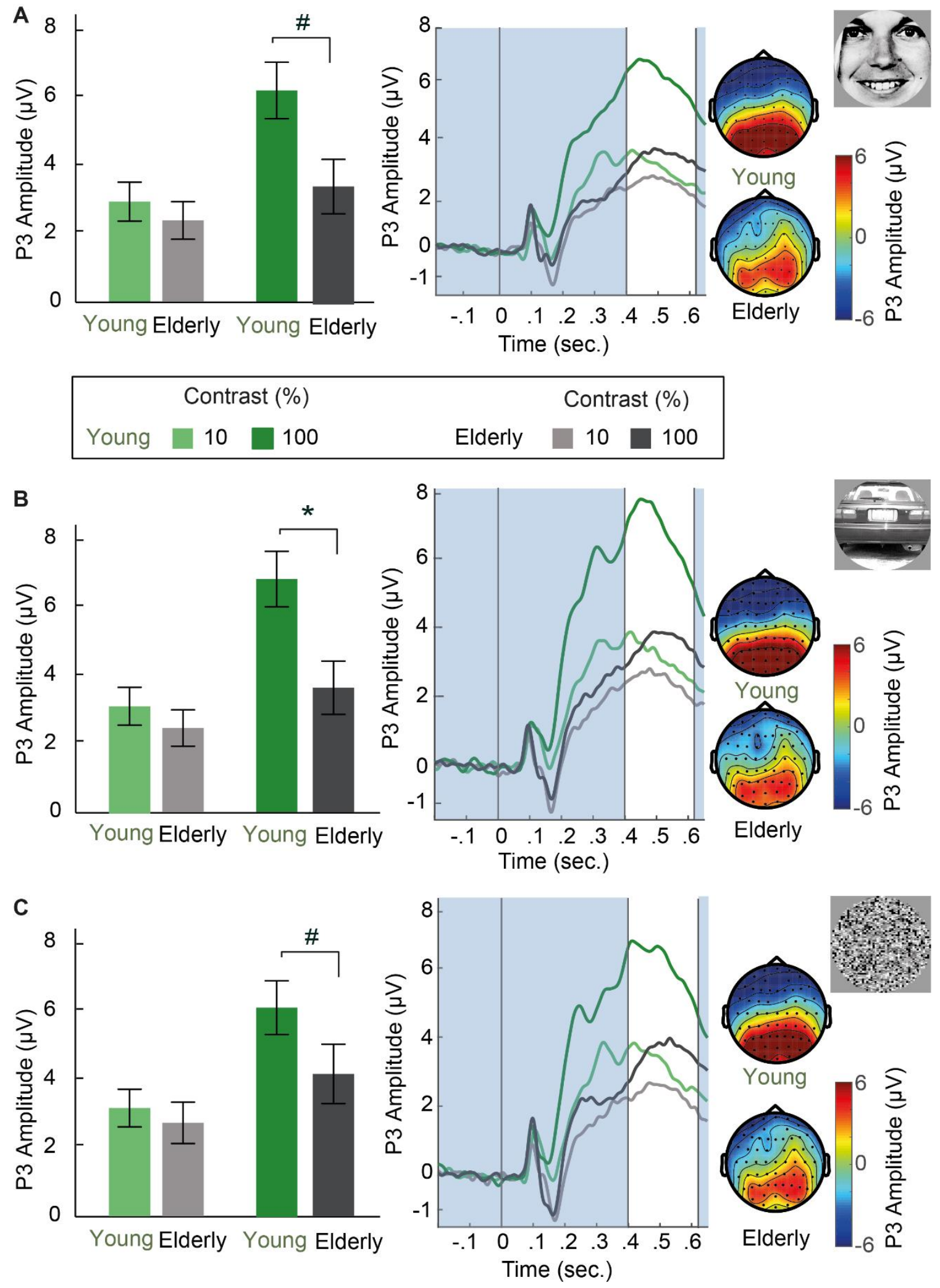

Figure 3.11: Differences in P3 Amplitude. P3 Amplitude derived from the electrodes P3, P4, separated by stimulus type, A) face, B) car, C) scrambled image and by subject group, Healthy Young $(N=22)$ in green and Healthy Elderly $(N=24)$ in gray. On the left in all 
panels, means of the P3 amplitude across subjects in each group are shown. Error bars denote S.E.M. across subjects. In the center P3 amplitude and on the right the spatial distribution of P3 Amplitude for the high contrast condition is shown. ** denotes a significant difference between Healthy Young and Healthy Elderly, $p<0.005$. ${ }^{*}$ denotes a significant difference between Healthy Young and Healthy Elderly, $p<0.008$ (black star as assessed by ANOVA and post-hoc t-tests). \# denotes significant difference without Bonferroni correction, $\mathrm{p}<0.05$ (assessed by ANOVA and post-hoc t-tests). On the right mean EEG activity is shown in green for Healthy Young $(N=22)$ and in grey for Healthy Elderly $(\mathrm{N}=24)$.

\subsubsection{Correlation of Event related Potentials and cognitive measurements}

To investigate whether $\mathrm{P} 1$ and $\mathrm{P} 3$ amplitudes would show age related cognitive changes we correlated P1 and P3 amplitude with cognitive and motor measures expecting lower amplitude being related to lower cognitive performance, longer times for task solving and more variable performance on the task in Healthy Elderly (Hsieh \& Lin, 2017; Speer \& Soldan, 2015). In Healthy Young and Healthy Elderly lower P1 amplitude in response to faces and cars in high and low contrast were related to higher intraindividual reaction time variability of the Stroop task for the incongruent condition in the Healthy Elderly. However, after removing 4 outliers scoring 2 SD below the mean the correlations between P1 amplitude and CVRT of the Stroop task for the incongruent condition did not remain significant $(p>0.15)$. After removing one outlier scoring 2 SD above the mean within the group intra-individual reaction time variability of the Stroop task for the congruent condition did not correlate with P1 amplitude in Healthy Young ( $p$ $>0.06$ ) (Supplementary Figure S3.1) 


\subsubsection{Time-frequency bands}

To investigate age differences in power change of different frequency bands we compared Healthy Young and Healthy Elderly expecting higher change in theta power in Healthy Elderly reflecting cognitive changes (Lithfous et al., 2015). Figure $\mathbf{3 . 1 1}$ shows power changes in alpha, beta, gamma and theta frequency bands after stimulus presentation. Theta power is higher in Healthy Elderly compared to Healthy Young (Young: $0.39 \%$, Elderly: $0.83 \%, F(1,44)=7.23, p=0.01$ ). As shown in Figure 3.12 (high contrast) and Supplementary Figure S.3.2 (low contrast) Healthy Elderly had increased power change in theta for high Contrast condition for faces images and scrambled compared to Healthy Young. This is supported by the interaction effect of "Contrast x Category x Group" and Bonferroni corrected comparisons, $p<0.0083$ (Table 3.4) $(F(1,44)=7.09, p=0.004$; Contrast $10 \%$ : faces $t(44)=-1.26, p=0.21$; cars $t(44)=-1.30, p=0.19$; scrambled $t(44)=-1.93, p=0.06$; Contrast $100 \%$ : faces $t(44)=-$ 2.96, $p=0.006$, cars $\mathrm{t}(44)=-1.97, \mathrm{p}=0.06$; scrambled $\mathrm{t}(44)=-3.68, \mathrm{p}=0.001$ ). 

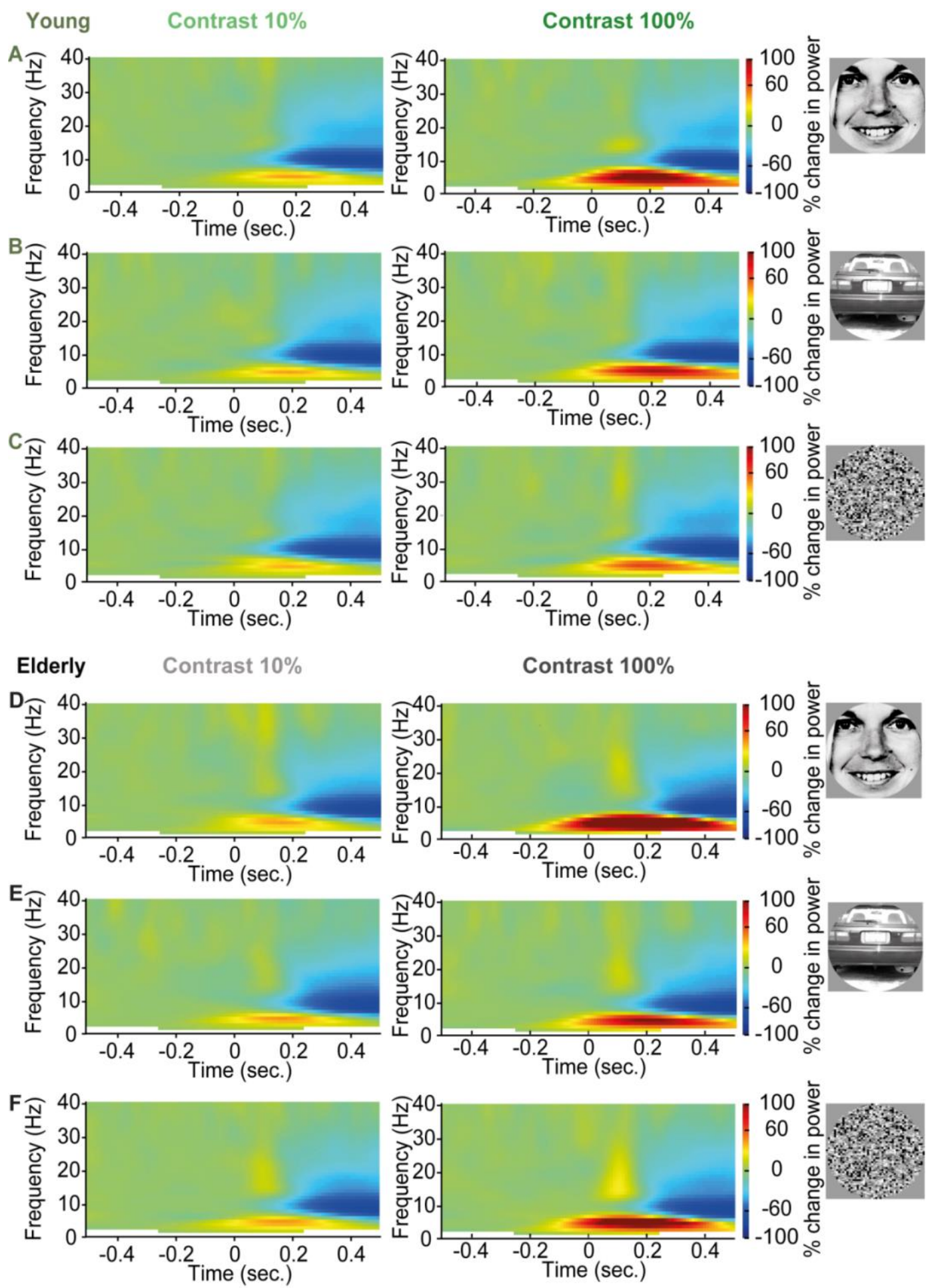

Figure 3.11: Time frequency spectra for high and low contrast condition in Healthy Young and Healthy Elderly. Changes in frequency power displayed on the y axis, ranging from $500 \mathrm{~ms}$ before stimulus onset at 0 seconds displayed on the $x$ axis and $500 \mathrm{~ms}$ after stimulus onset separated group for stimulus type A) faces, B) cars and C) scrambled, Healthy Young and Healthy Elderly D) faces, E) cars, F) scrambled, right high contrast condition and low contrast condition on the left. 
Table 3.4: Spectral Power Change: Repeated measures mixed ANOVA of power change in alpha, beta, gamma and theta frequency bands with the factors contrast, category and group of Healthy Young $(\mathrm{N}=22)$ and Healthy Elderly $(\mathrm{N}=24)$

\begin{tabular}{|c|c|c|c|c|}
\hline & Alpha & Beta & Gamma & Theta \\
\hline & $F(p)$ & $F(p)$ & $F(p)$ & $F(p)$ \\
\hline Contrast & $14.41(=0.0004)^{\star *}$ & $1.44(=0.24)$ & $0.001(=0.98)$ & $52.09(<0.0001)^{\star *}$ \\
\hline Category & $8.33(=0.001)^{\star *}$ & $0.44(=0.65)$ & $3.12(=0.052)$ & $25.82(<0.0001)^{\star \star}$ \\
\hline Group & $1.08(=0.31)$ & $0.42(=0.51)$ & $0.99(=0.33)$ & $7.23(=0.010)^{*}$ \\
\hline $\begin{array}{l}\text { Contrast X } \\
\text { Group }\end{array}$ & $0.19(=0.66)$ & $0.001(=0.98)$ & $0.42(=0.52)$ & $11.23(=0.002)^{\star \star}$ \\
\hline $\begin{array}{l}\text { Category x } \\
\text { Group }\end{array}$ & $1.41(=0.25)$ & $3.17(=0.047)^{*}$ & $0.83(=0.43)$ & $8.22(=0.002)^{\star \star}$ \\
\hline $\begin{array}{l}\text { Contrast x } \\
\text { Category x } \\
\text { Group }\end{array}$ & $1.00(=0.37)$ & $1.56(=0.22)$ & $0.75(=0.46)$ & $7.09(=0.004)^{\star \star}$ \\
\hline $\begin{array}{l}\text { Contrast x } \\
\text { Category }\end{array}$ & $9.09(=0.0003)^{\star \star}$ & $0.72(=0.49)$ & $0.15(=0.84)$ & $23.69(<0.0001)^{\star \star}$ \\
\hline $\begin{array}{l}\text { Between-sub } \\
\text { Within-subjec } \\
{ }^{*} p<0.05 ;{ }^{* *}\end{array}$ & $\begin{array}{l}\text { tor: Group: } 2 \text { Level } \\
\text { s: Category: } 3 \text { leve } \\
5\end{array}$ & $\begin{array}{l}\text { Young, Elderly } \\
\text { face, car, Contr }\end{array}$ & 15 & ast $100 \%$ \\
\hline
\end{tabular}




\begin{tabular}{|c|c|}
\hline Young & $\begin{array}{l}\text { - alpha } \Lambda \text { gamma } \\
\text { beta theta }\end{array}$ \\
\hline
\end{tabular}
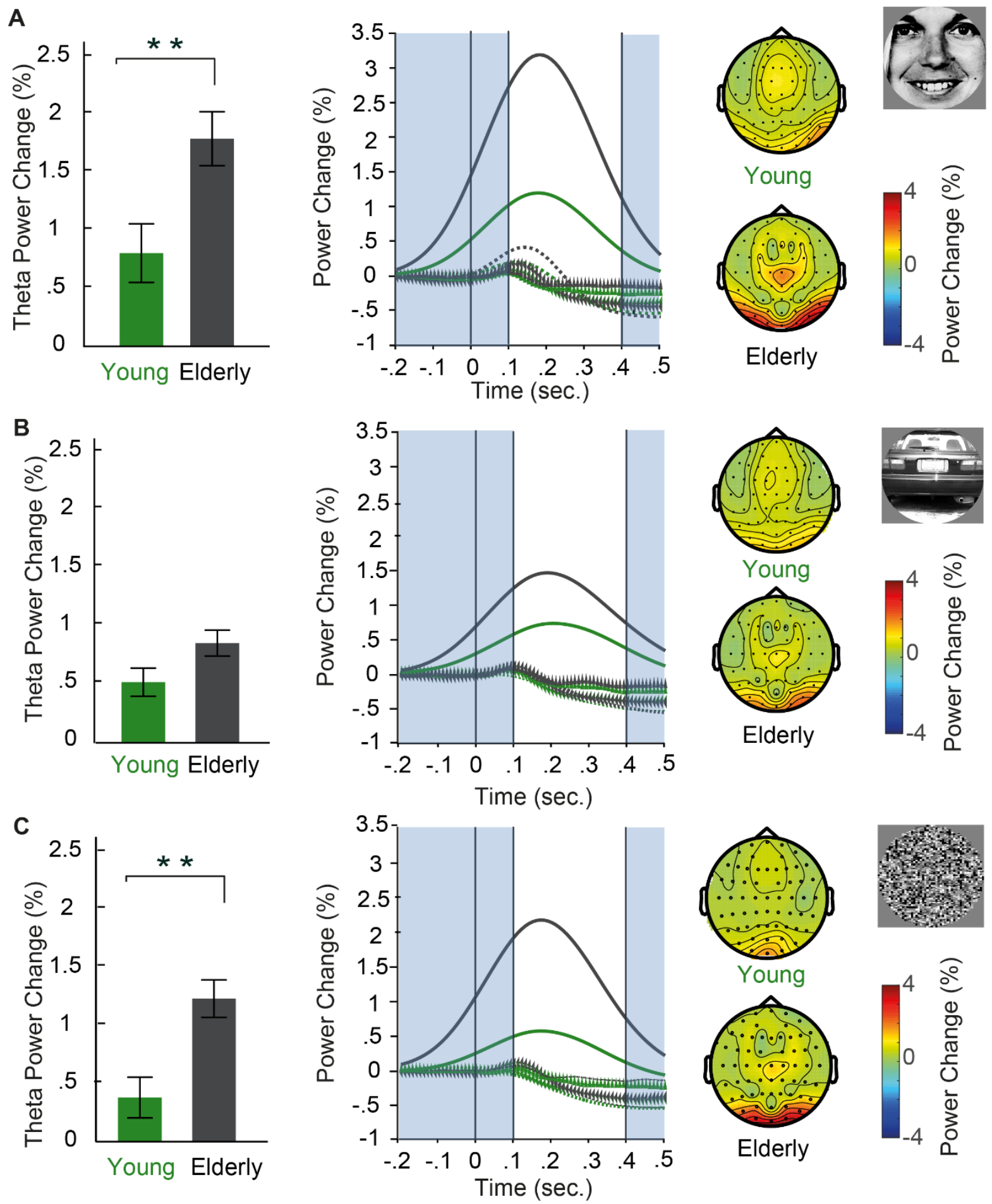

Figure 3.12: Group Differences in change of Theta power of the high contrast condition. Average percentage of Theta power change, separated by stimulus type, A) face or B) car, and C) scrambled image and by subject group, Healthy Young $(N=22)$ in green and Healthy Elderly $(N=24)$ in gray. On 
the left in all panels, means of the theta power change across subjects in each group are shown. Error bars denote S.E.M. across subjects. In the center power change in different frequency bands of interest such as alpha, beta, gamma and theta and on the right spatial distribution of change in theta power of the high contrast condition are shown. * denotes a significant difference, $\mathrm{p}<0.05$ (black star as assessed by the ANOVA and post-hoc tests) ** denotes a significant difference, $p<0.005$ (black star as assessed by the ANOVA and post-hoc tests).

\subsubsection{Correlation of change in Theta power and cognitive measurements}

To test wether differences in power change of theta reflect changes in cognition we correlated power change in theta and scores on cognitive tests such as the Trail Making Test and Stroop Test expecting higher power change being related to worse performance (Lithfous et al., 2015). As shown in Figure 3.13 higher change in theta power correlates with lower variability of noncongruent Stroop condition in Healthy Elderly, which remain stable after removing 5 outliers that deviated \pm 2 SD from the mean within group $(p<0.04)$. 
A

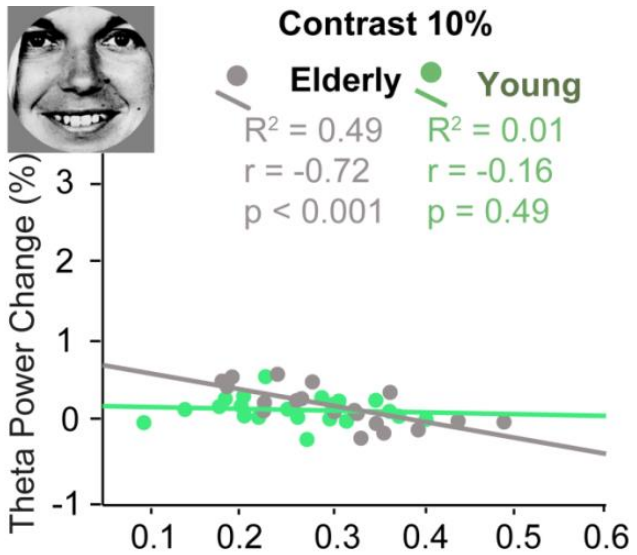

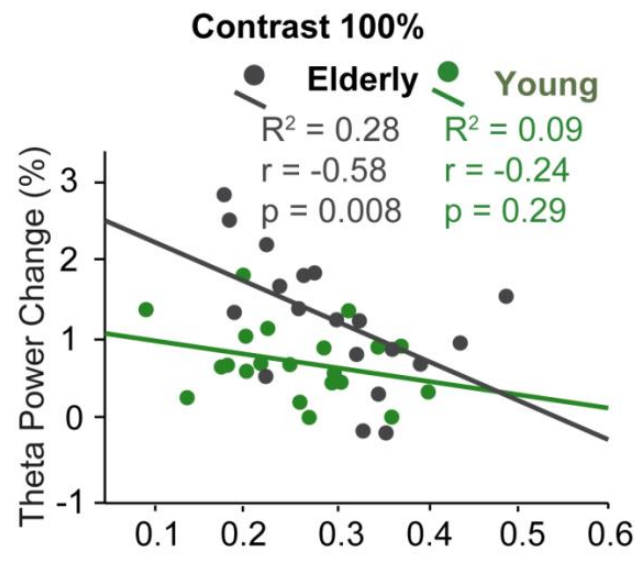

Coefficient of RT variability(CVRT) Stroop incongurent

B

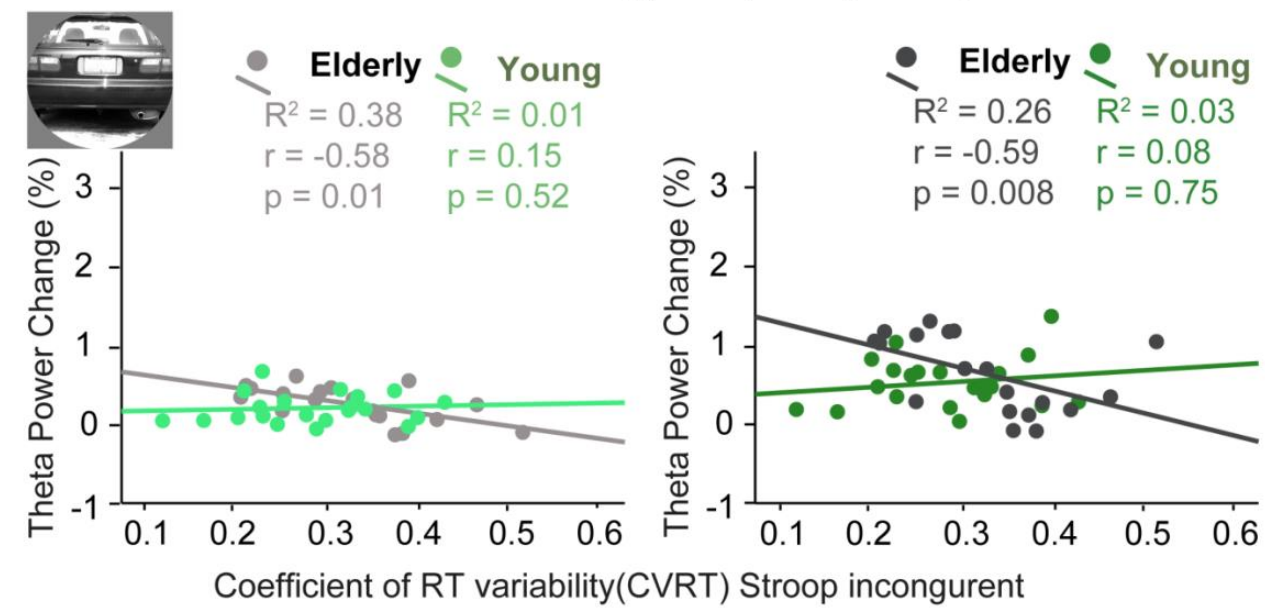

C

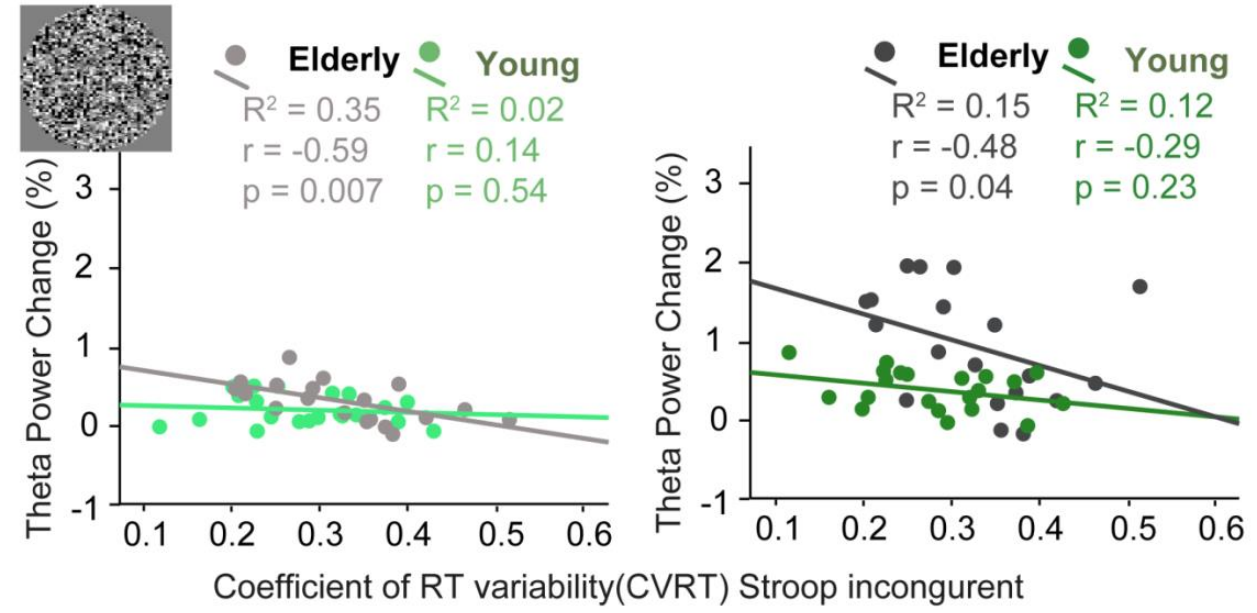

Figure 3.13: Association of change in Theta power and coefficient reaction time variability of Stroop incongruent condition. Correlation change in Theta power for A) faces B) cars and C) scrambled images (left) Contrast $10 \%$ and (right) Contrast $100 \%$ displayed on the $y$-axis and coefficient reaction time variability of Stroop task incongruent condition on the x-axis. Green dots show the individual values of Healthy Young volunteers ( $N=21$ (without outlier) and grey dots the individual values of Healthy Elderly volunteers ( $\mathrm{N}=19$ (without outlier)). 


\subsubsection{Variability Quenching}

To test age differences in neural variability we compared percent change between Healthy Young and Healthy Elderly expecting lower variability quenching in Healthy Elderly indicating variability quenching as a possible mechanism of cognitive changes and increase in behavioral variability. Figure 3.14 illustrates that in both groups, Healthy Young and Healthy Elderly volunteers, increased quenching 100 - 400 ms after stimulus presentation for the low contrast condition (Table 3.5), especially for faces and cars (Young: Contrast 10\% vs. Contrast 100\%: average: $\mathrm{t}(21)=-6.70, \mathrm{p}<0.0001$; faces: $\mathrm{t}(21)=-4.79, \mathrm{p}<0.0001$; cars: $\mathrm{t}(21)=-4.16, \mathrm{p}=0.0004$; scrambled: $\mathrm{t}(21)=-$ 2.56, $p=0.02$; Elderly: Contrast $10 \%$ vs. Contrast $100 \%$ : average: $t(23)=-4.57, p=$ 0.0001 ; faces: $t(23)=-3.13, p=0.005$; cars: $t(23)=-3.02, p=0.0061$; scrambled: $t(23)$ $=-0.65, p=0.52)$ Bonferroni corrected (for eight comparisons, four within each group) significance threshold was $p<0.00625$. We exploratory tested whether quenching was time dependent and tested separately three time intervals after stimulus presentation. All time windows showed comparable stable effects of contrast condition but no group differences (Table 3.5). Only the time window $200-300 \mathrm{~ms}$ after stimulus presentation showed an interaction effect of "Group x Contrast" $(F(1,44)=4.18, p=0.047)$. Posthoc comparisons showed that there is no difference between Healthy Elderly and Healthy Young for low and high contrast condition (Contrast 10\%: $t(44)=0.69, p=0.49$; Contrast $100 \%: \mathrm{t}(44)=16, \mathrm{p}=0.88)$. The interaction effect is explained by higher increase in variability quenching between high and low contrast condition in Healthy Young compared to Healthy Elderly $(t(44)=2.04, p=0.047)$ albeit not significant after Bonferroni correction for multiple comparisons $(p<0.016)$. 
Table 3.5: Variability Quenching: Repeated measures mixed ANOVA with the factors contrast, category and group of Healthy Young $(\mathrm{N}=22)$ and Healthy Elderly $(\mathrm{N}=24)$

\begin{tabular}{|c|c|c|c|c|}
\hline & $\begin{array}{l}\text { Variability } \\
\text { Quenching } \\
\text { 100-400 ms after } \\
\text { stimulus } \\
\text { presentation }\end{array}$ & $\begin{array}{l}\text { Variability } \\
\text { Quenching } \\
\text { 100-200 ms after } \\
\text { stimulus } \\
\text { presentation }\end{array}$ & $\begin{array}{l}\text { Variability } \\
\text { Quenching } \\
200-300 \text { ms after } \\
\text { stimulus } \\
\text { presentation }\end{array}$ & $\begin{array}{l}\text { Variability } \\
\text { Quenching } \\
300-400 \text { ms after } \\
\text { stimulus } \\
\text { presentation }\end{array}$ \\
\hline & $F(p)$ & $F(p)$ & $F(p)$ & $F(p)$ \\
\hline $\begin{array}{l}\text { Contrast } \\
\text { Category }\end{array}$ & $\begin{array}{l}\begin{array}{l}43.37 \\
(<0.0001)^{\star *}\end{array} \\
1.81(=0.17)\end{array}$ & $\begin{array}{l}\begin{array}{l}30.79 \\
(<0.0001)^{\star *}\end{array} \\
3.62(=0.03)^{\star}\end{array}$ & $\begin{array}{l}\begin{array}{l}34.34 \\
(<0.0001)^{\star *}\end{array} \\
2.56(=0.08)\end{array}$ & $\begin{array}{l}\begin{array}{l}30.74 \\
(<0.0001)^{* *}\end{array} \\
4.62(=0.01)^{*}\end{array}$ \\
\hline Group & $0.34(=0.56)$ & $2.59(=0.11)$ & $0.05(=0.82)$ & $0.03(=0.87)$ \\
\hline $\begin{array}{l}\text { Contrast X } \\
\text { Group }\end{array}$ & $3.39(=0.07)$ & $1.02(=0.32)$ & $4.18(=0.047)^{\star}$ & $2.92(=0.09)$ \\
\hline $\begin{array}{l}\text { Category } \mathrm{x} \\
\text { Group }\end{array}$ & $1.53(=0.22)$ & $1.19(=0.31)$ & $0.23(=0.78)$ & $2.85(=0.07)$ \\
\hline $\begin{array}{l}\text { Contrast X } \\
\text { Category x } \\
\text { Group }\end{array}$ & $0.03(=0.97)$ & $0.12(=0.89)$ & $0.08(=0.92)$ & $0.07(=0.91)$ \\
\hline $\begin{array}{l}\text { Contrast x } \\
\text { Category }\end{array}$ & $5.39(=0.007)^{\star}$ & $3.23(=0.046)^{\star}$ & $7.01(=0.002)^{\star \star}$ & $4.96(=0.01)^{\star}$ \\
\hline
\end{tabular}


Young
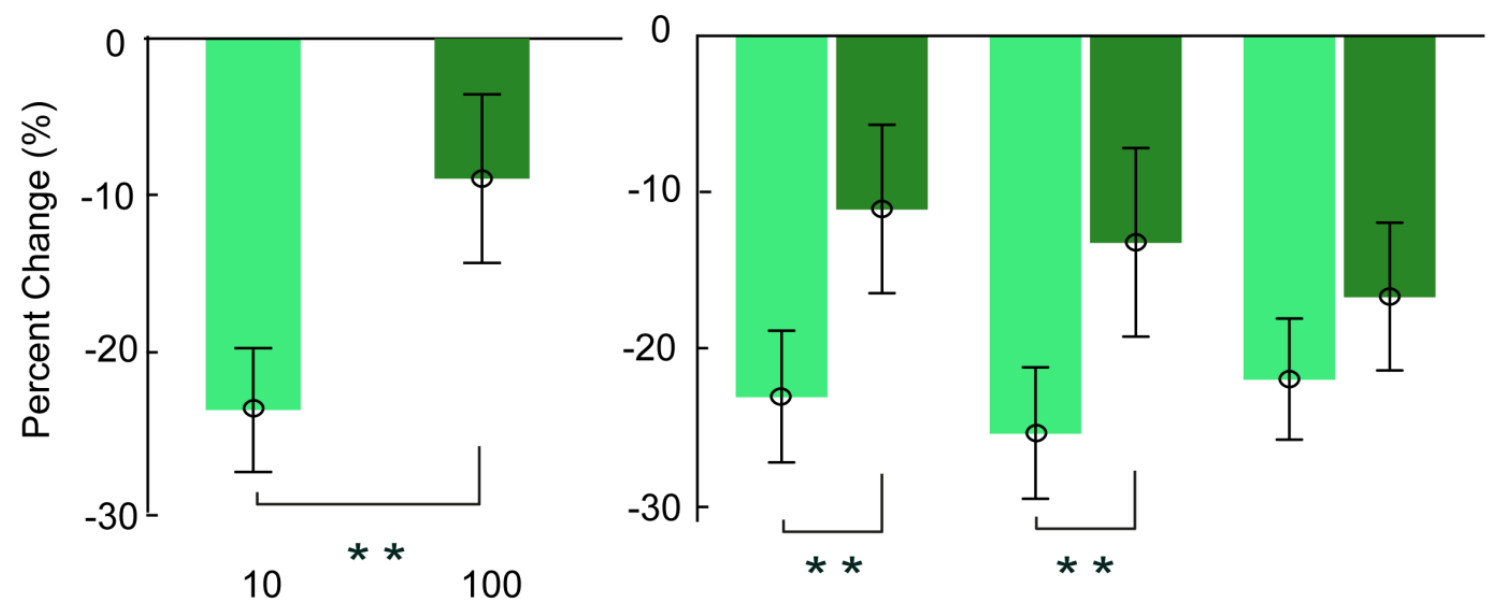

Contrast
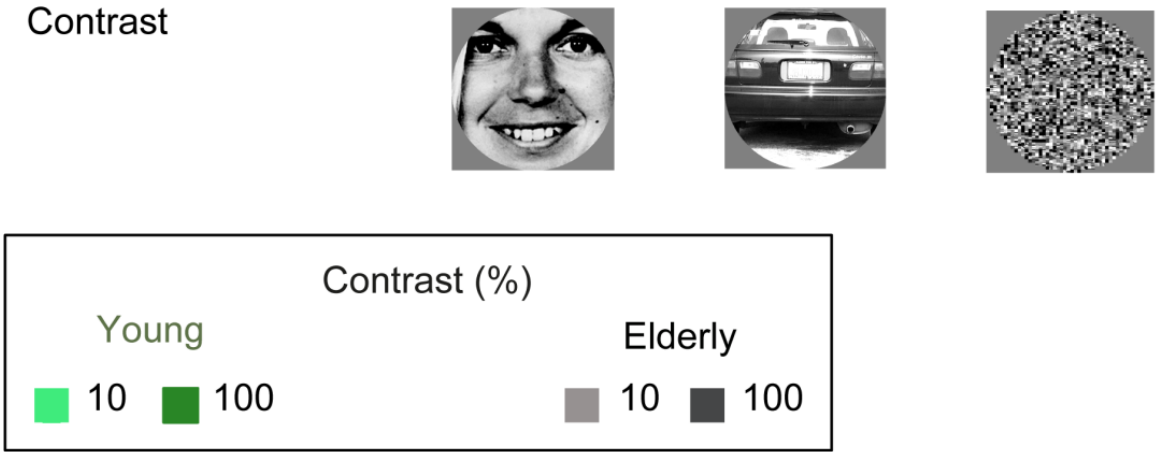

\section{Elderly}
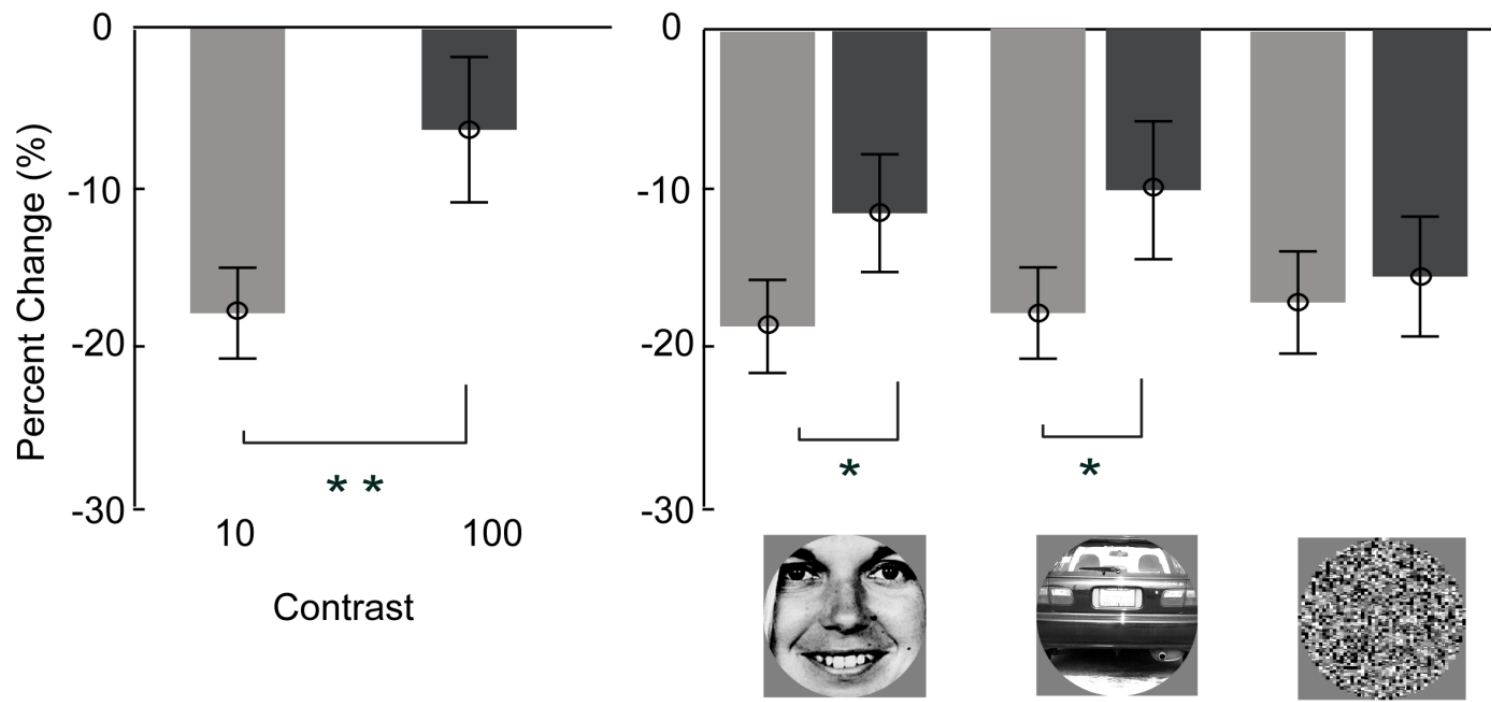

Figure 3.14: Variability Quenching $100-400 \mathrm{~ms}$ after stimulus presentation. Difference in percent change in high and low contrast condition in Healthy Young ( $\mathrm{N}=22$ ) (upper) and Healthy Elderly $(\mathrm{N}=24)$ (lower). *denotes Bonferroni corrected significance threshold $p<0.00625{ }^{* *}$ denotes a significant difference, $p<0.005$ (black star as assessed by the ANOVA). 


\subsubsection{Correlation of Variability Quenching and cognitive measurements}

To investigate whether neural variability is related to cognitive differences we correlated percent change of variability quenching with scores on the Trail Making Test expecting higher variability quenching being related to less time required to solve the task (Arazi, Censor, et al., 2017). As shown in Figure 3.15 in Healthy Elderly subjects increased time to solve Trail Making Test part A correlated with increased quenching for the high and low contrast condition (Contrast 10\%: $r_{s}=-0.49, p=0.02$; Contrast $100 \% ; r_{s}=-$ $0.49, p=0.01)$. The correlation remains stable after removing 7 outliers scoring $\pm 2 S D$ away from the mean, between Trail Making Test part $A$ and variability quenching of the low contrast condition in Healthy Elderly (Contrast $10 \%: r_{s}=-0.49, p=0.03$; faces: $r_{s}=-$ $0.39, p=0.10$; cars: $r_{s}=-0.50, p=0.03$, scrambled: $r_{s}=-0.56, p=0.01$; Contrast $100 \%$ : $r_{s}=-0.39, p=0.09 ;$ faces: $r_{s}=-0.43, p=0.07$, cars: $r_{s}=-0.27, p=0.27$, scrambled: $r_{s}=$ $-0.52, p=0.02)$. Increased variability quenching in response to high contrast scrambled images is related to longer times to solve the Trail Making Test part A and part B in Healthy Elderly (TMT A: $r_{s}=-0.62, p=0.001$, TMT B: $r_{s}=-0.43, p=0.03$ ). 
A
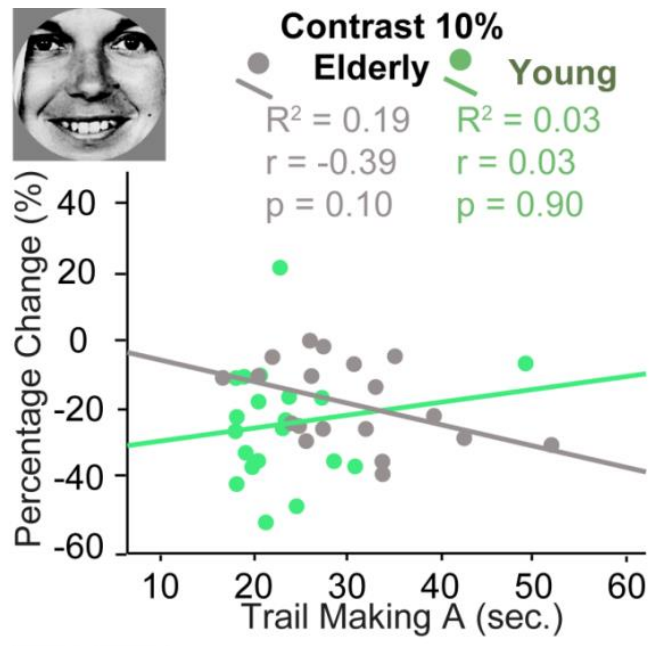

B

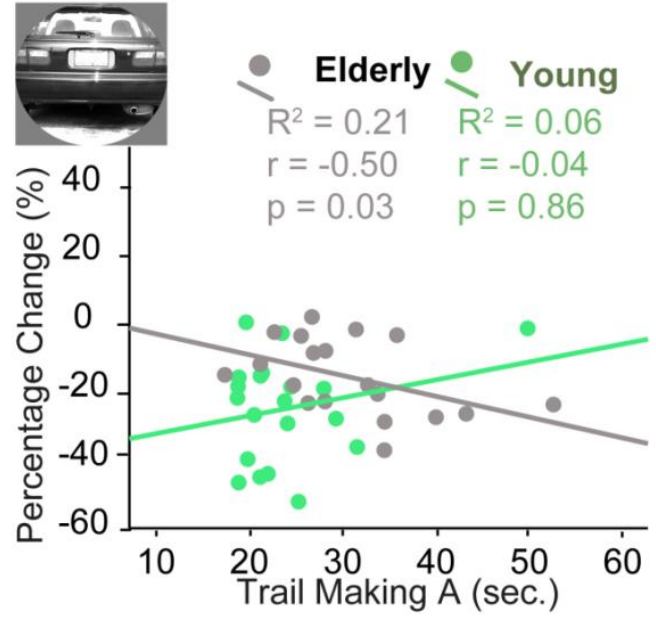

C

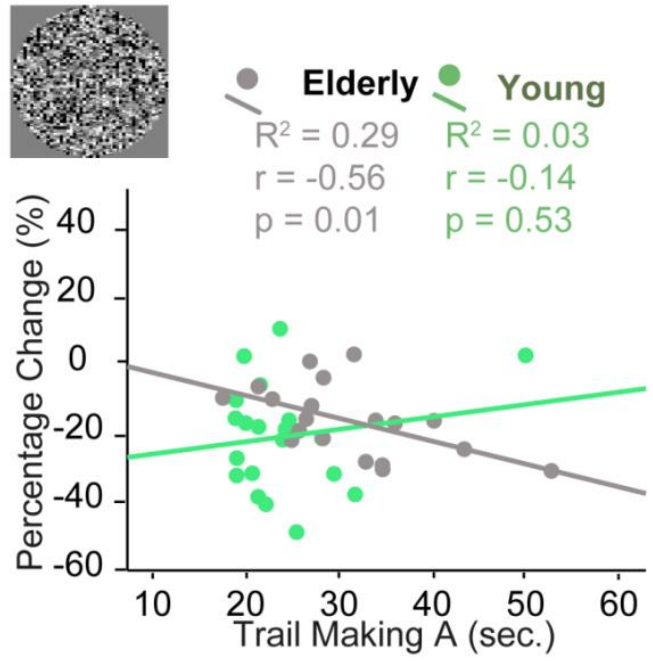

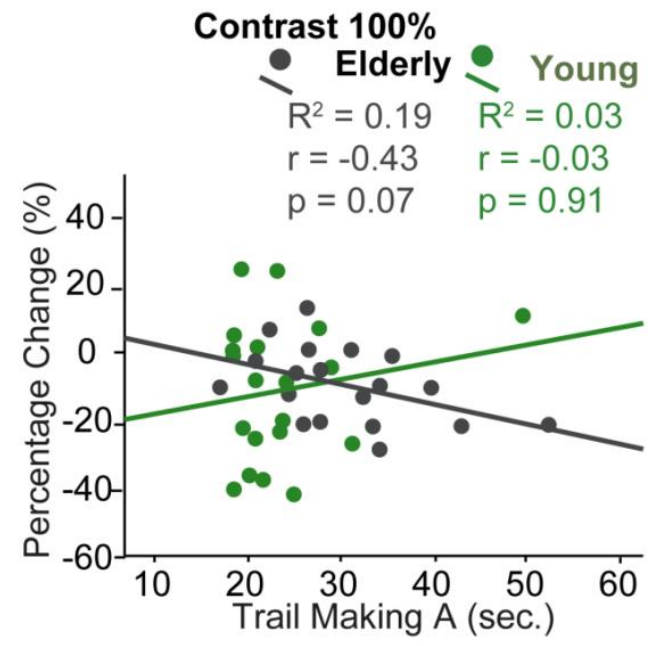
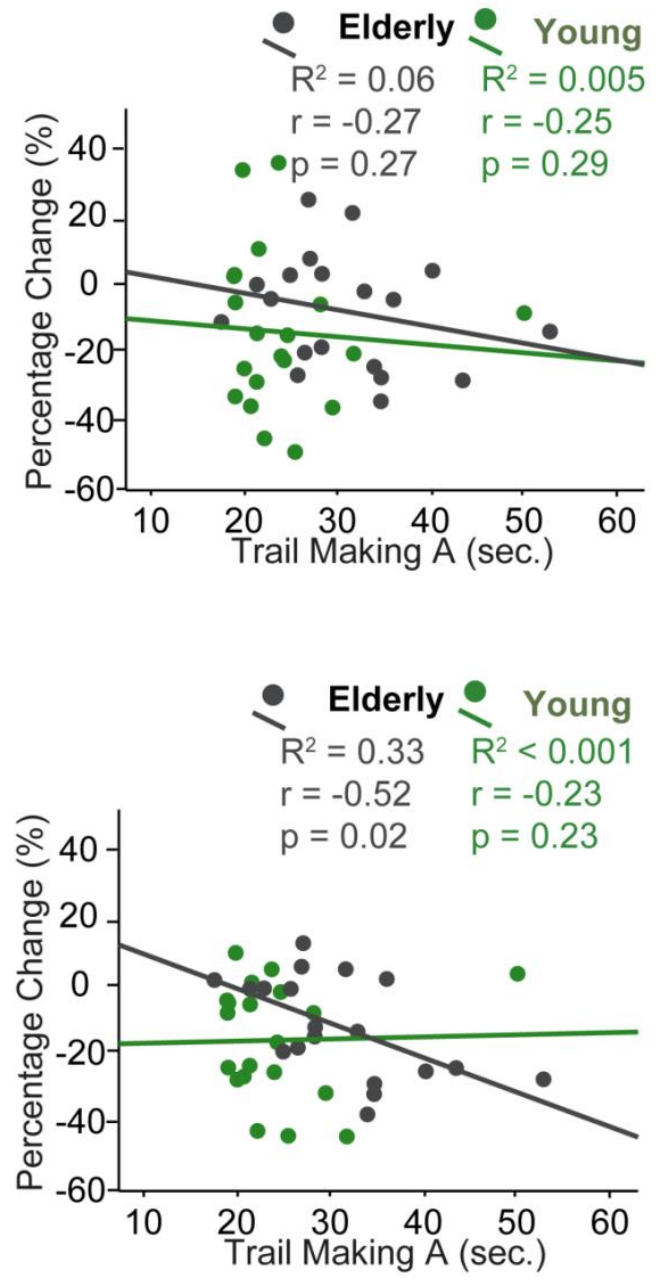

Figure 3.15: Relation of Variability quenching and Trail Making Test part A. A) Correlation between Percent change for Contrast 10\% A) faces B) cars and C) scrambled images left Contrast $10 \%$ and right Contrast $100 \%$ displayed on the $y$-axis and time solving Trail Making Test part A on the $x$-axis. Green dots show the individual values of Healthy Young volunteers $(\mathrm{N}=20$ (without outlier)) and grey dots the individual values of Healthy Elderly volunteers $(\mathrm{N}=19$ (without outlier)). 
In Healthy Young volunteers increased time to solve the slightly more complex Trail Making Test part B was related to reduced quenching in low contrast condition and for scrambled images in the high and low contrast condition (Contrast 10\%: $r_{s}=0.50, p=$ 0.02; Contrast $100 \%: r_{s}=0.52, p=0.01$ ). After excluding seven influential observations, $\pm 2 S D$ deviant from the average within the group only the correlation for higher quenching of the average of the low contrast stimuli and low contrast facial images and less time for the TMT part B was significant in Healthy Young volunteers (Contrast 10\%: $r_{s}=45, p=0.044:$ faces: $r_{s}=0.52, p=0.02 ;$ cars: $r_{s}=0.39, p=0.08$; scrambled: $r_{s}=$ $0.44, p=0.051$; Contrast $100 \%: r_{s}=0.32, p=0.16$, faces: $r_{s}=25, p=0.28$; cars $=0.26$, $p=0.26$; scrambled: $\left.r_{s}=43, p=0.06\right)$ (Figure 3.16). 
A

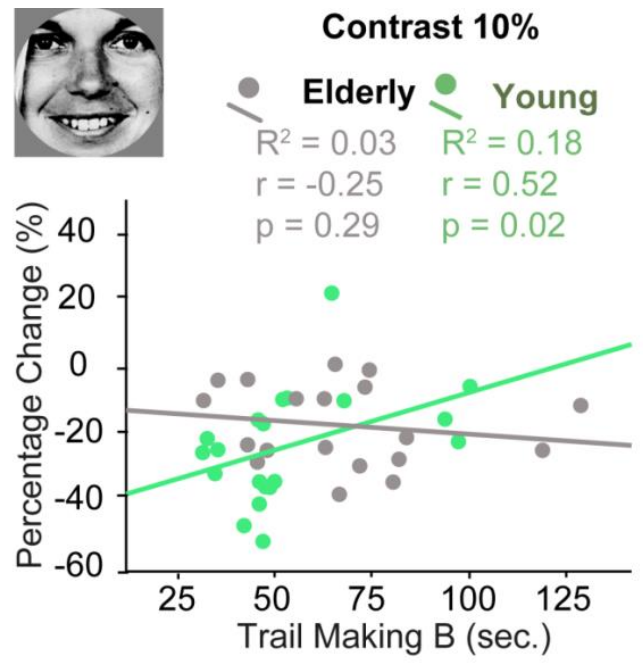

B

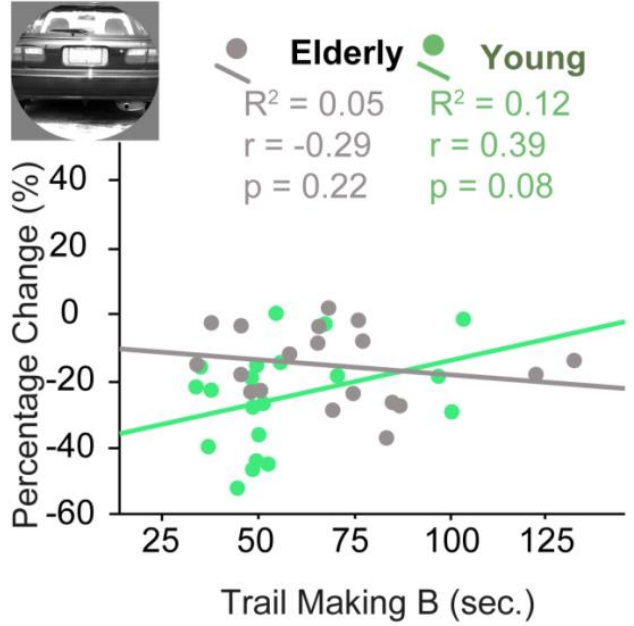

C

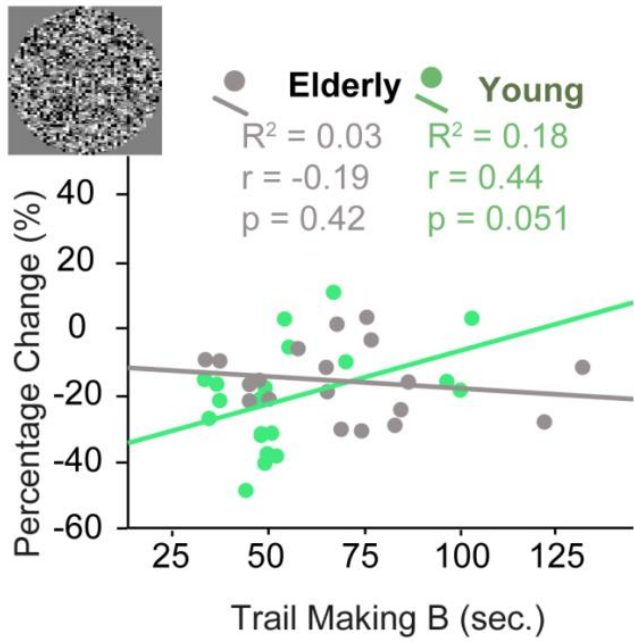

Contrast $100 \%$
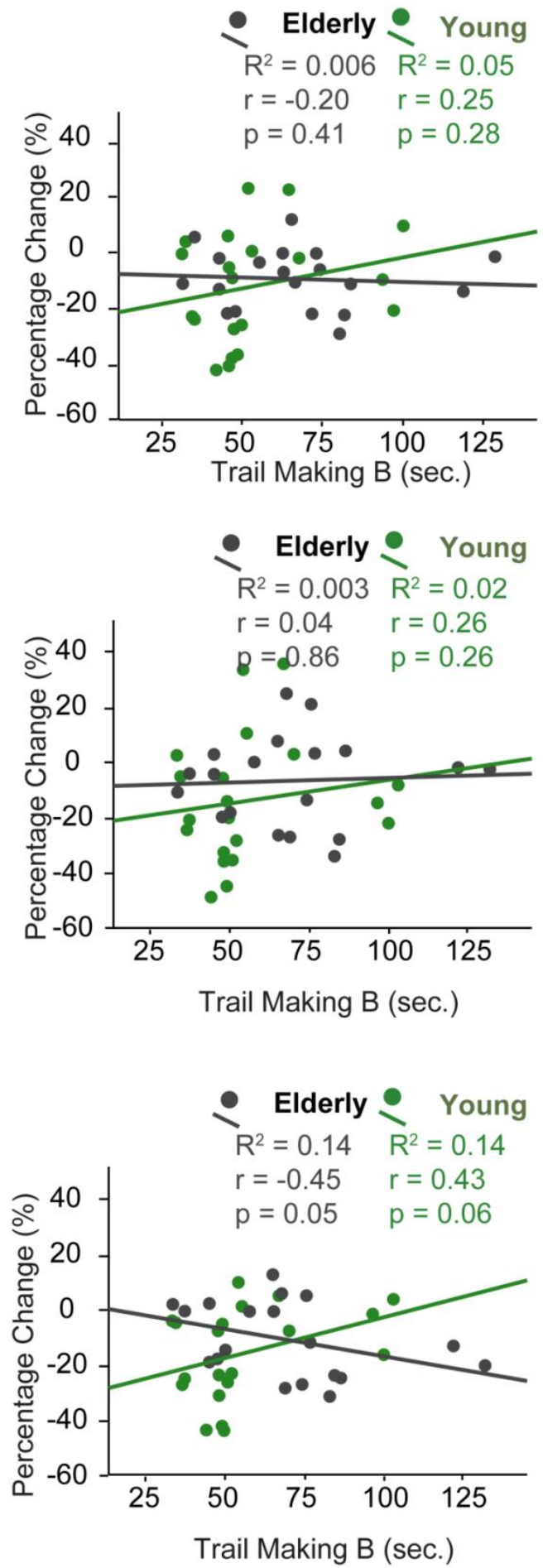

Figure 3.16. Relation of Variability Quenching and Trail Making Test part B. Correlation between Percent change for Contrast $10 \%$ A) faces B) cars and C) scrambled images left Contrast $10 \%$ and right Contrast $100 \%$ displayed on the $y$-axis and time solving Trail Making Test part A on the $x$-axis. Green dots show the individual values of Healthy Young volunteers $(N=20$ (without outlier)) and grey dots the individual values of Healthy Elderly volunteers $(N=19$ (without outlier)). 
To test whether variability quenching is related to behavioral variability we correlated CVRT and percentage change of variability quenching. Behavioral variability and variability quenching were not correlated in both groups. To investigate whether correct responses were related to increased variability quenching we correlated proportion correct responses with percentage change of variability quenching (Arazi, Censor, et al., 2017). Only proportion correct categorization for high contrast faces were related to variability quenching in Healthy Young $\left(r_{s}=0.47, p=0.03\right)$. However, removing three influential observations scoring 2SD below the mean the correlation did not remain stable $\left(r_{s}=0.45, p=0.051\right)$.

\subsubsection{Heart Rate Variability}

To investigate whether HRV reflects changes in age we tested differences in HRV expecting higher HRV in Healthy Young. In accordance with previous research HRV parameter such as RMSSD, High frequency power and low frequency power were reduced in Healthy Elderly (Shaffer et al., 2014) (Table 3.6, Figure 3.17).

Table 3.17: Heart Rate Variability: Group differences in Heart Rate Variability between Healthy Young $(\mathbf{N}=20)$ and Healthy Elderly $(N=22)$

\begin{tabular}{llcl} 
& Pre task Resting state & During the task & Post task Resting state \\
\hline & $\mathrm{t}(\mathrm{p})$ & $\mathrm{t}(\mathrm{p})$ & $\mathrm{t}(\mathrm{p})$ \\
\hline RMSSD & $1.83(=0.07)$ & $3.45(=0.001)^{\star \star}$ & $2.88(=0.006) \#$ \\
\hline LF power (ms2) & $1.55(=0.13)$ & $31.28(<0.0001)^{\star *}$ & $1.57(=0.12)$ \\
\hline HF power (ms2) & $1.08(=0.29)$ & $2.64(=0.01) \#$ & $2.31(=0.03) \#$ \\
\hline LF / HF ratio (ms2) & $-0.89(=0.39)$ & $0.85(=0.40)$ & $-0.82(=0.42)$ \\
$\# \mathrm{p}<0.05$ Bonferroni corrected significance ${ }^{*} \mathrm{p}<0.0042 ;{ }^{* *} \mathrm{p}<0.001$ &
\end{tabular}




\section{A Pre task Resting State}
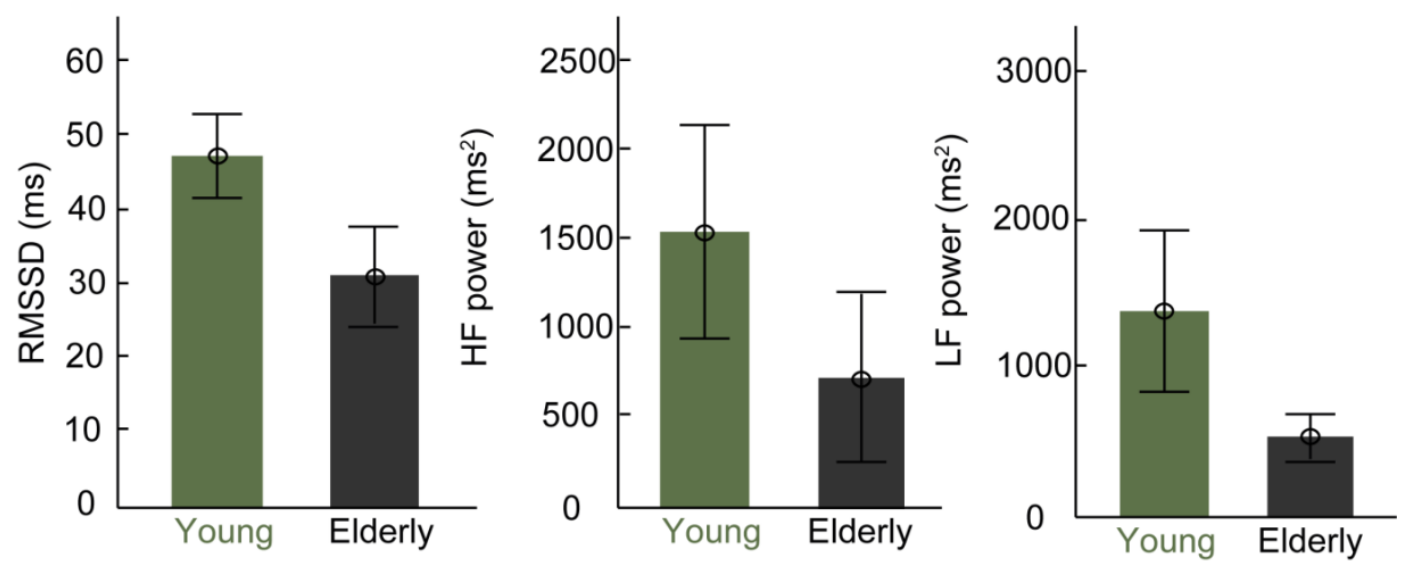

\section{B During the task}
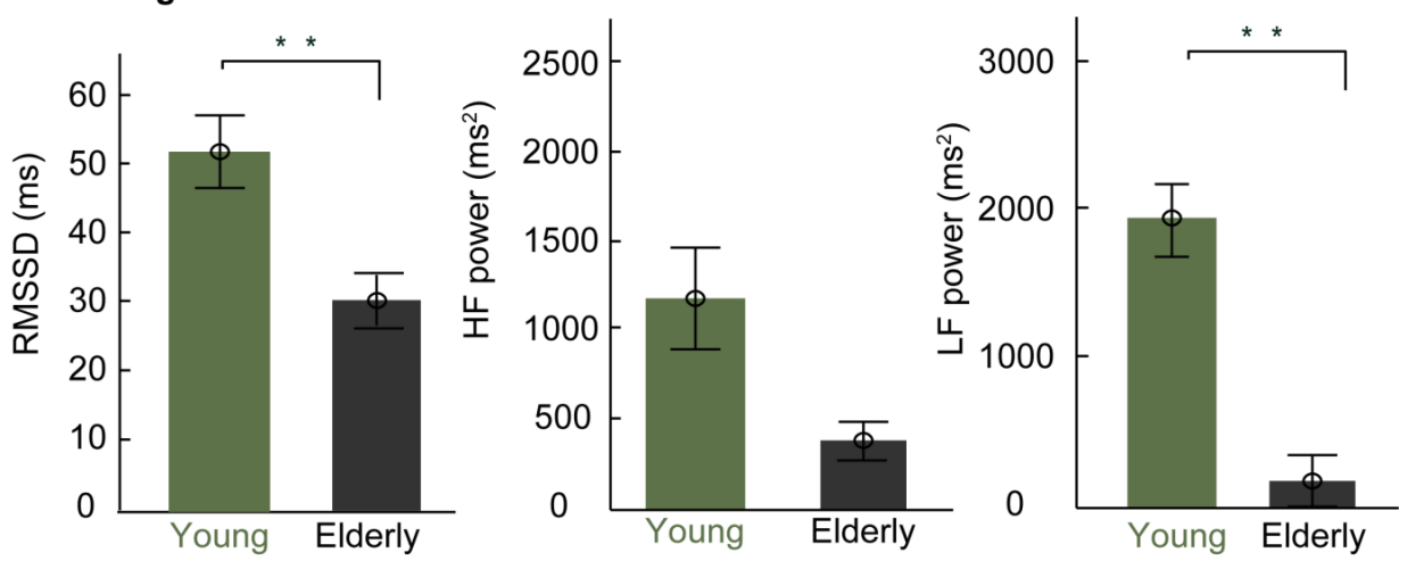

C Post task Resting State
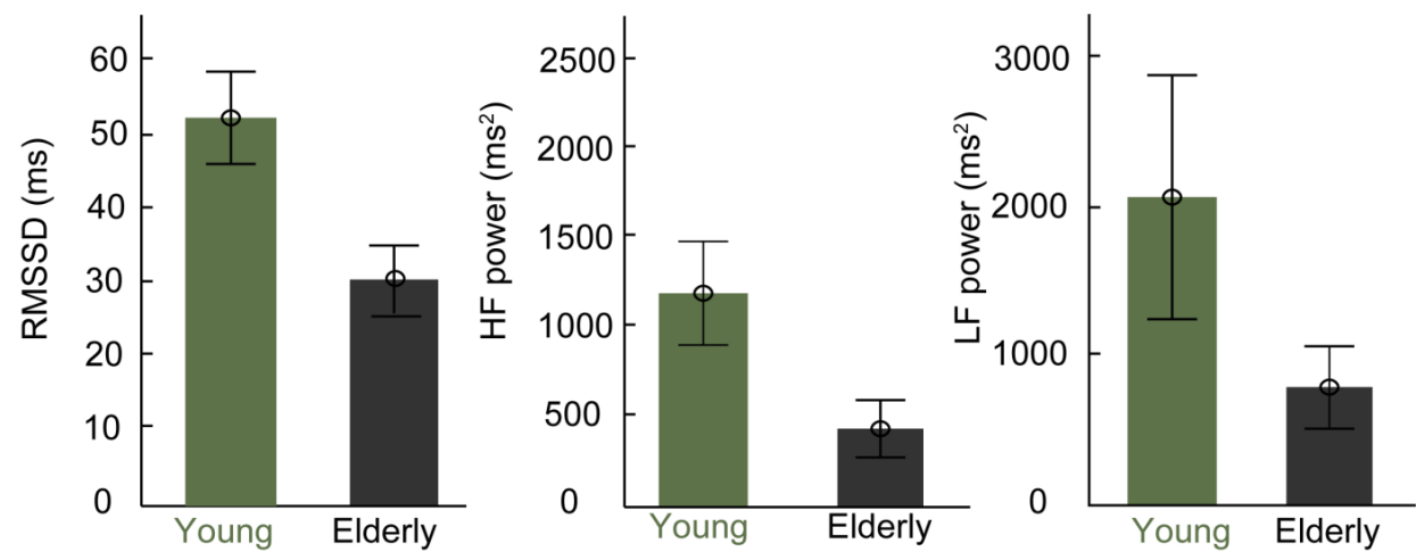

Figure 3.17: Heart rate variability differences between Healthy Young and Healthy Elderly. (A) Root Mean Square of Successive Differences (left) High Frequency power (middle) and Low frequency power (right) for Healthy Young $(\mathrm{N}=20)$ green bars and Healthy Elderly $(\mathrm{N}=24)$ black bars. Error bars show the standard error of the mean. ${ }^{* *}$ denotes a significant difference between Healthy Young and Healthy Elderly, $p<0.005$ (black star as assessed by t-test) in the Resting state (2 min.) before the task (B) during the task (C) in the Resting state (2 min.) after the task. 


\subsubsection{Correlation of Heart Rate Variability, cognitive measurements and task measurements}

To investigate whether HRV is related to cognitive changes in Healthy Elderly and in Healthy Young we correlated different HRV parameters with scores on different cognitive tests such as the Trail Making Test expecting higher cognitive performance being related to higher HRV (Thayer et al., 2009). Lower RMSSD during the task correlated with faster solving of the Trail Making Test part A in the Healthy Elderly $\left(r_{s}=\right.$ $0.48, p=0.02)$. This correlation did not remain stable after removing 2 outlier scoring $\pm 2 S D$ away from the average within the group $\left(r_{s}=0.31, p=0.81\right)$. Increased LF power is related to faster solving of the Trail Making Test part A in the Healthy Young $\left(r_{s}=-\right.$ $0.56, p=0.01$ ) which did not remain stable after removing 2 influential observation scoring 2SD above the mean of within the group $\left(r_{s}=-0.46, p=0.058\right)$.

To investigate whether HRV reflects behavioral changes we correlated different HRV parameters with task measures of behavioral variability expecting higher HRV being related to higher intra- individual variability. First, we correlated average coefficient of recognition time variability separately for high and low contrast stimuli with HRV measures. In both groups, Healthy Young and Healthy Elderly, both conditions did not correlate with the HRV measures ( $p>0.061)$. However, after separating the conditions for the different type of stimuli, higher behavioral variability in response in low contrast cars and scrambled images was related to higher RMSSD in Healthy Young (Contrast 10\%: cars: $r_{s}=0.47, p=0.036$; scrambled: $\left.r_{s}=0.45, p=0.045\right)$. Higher behavioral variability in response to scrambled images was correlated with higher LF power in Healthy Young $\left(r_{s}=0.51, p=0.02\right)$. After removing 2 influential observations scoring 
2SD above the mean within the group higher RMSSD correlated with increased intraindividual variability in response to low contrast cars in Healthy Young (RMSSD: $r_{s}=$ $0.48, p=0.045$ (Supplementary Figure S3.3). In Healthy Elderly, after removing four influential observation scoring 2 SD above the average within the group, higher RMSSD, LF power and HF power correlated with lower intra-individual variability in response to high contrast cars (RMSSD: $r_{s}=-0.54, p=0.02$ (Supplementary Figure S3.3); LF: $\left.r_{s}=-0.52, p=0.03 ; H F: r_{s}=-0.65, p=0.004\right)$. However, given that behavioral variability for only one high contrast condition correlated with HRV this results seems unreliable.

To test whether ERP's are related to differences in cardiac variability we correlated amplitude of P1 and P3 with HRV parameters expecting higher amplitudes to be related to higher heart rate variability. First, we correlated average amplitude of the P1 and P3 component separately for high and low contrast stimuli with HRV measures. In both groups, Healthy Young and Healthy Elderly, both conditions did not correlate with the HRV measures $(p>0.15)$. However, after separating the conditions for the different type of stimuli, only higher P1 amplitude in response to low contrast faces was related to lower HF power in Healthy Young $\left(r_{s}=-0.46, p=0.043\right)$ which did not remain stable after removing two outliers scoring 2 SD above the average within the group $\left(r_{s}=-0.46\right.$, $\mathrm{p}=0.06)$.

To test whether change in theta power is related to differences in HRV we correlated power change with HRV parameters expecting higher power change being related to lower HRV. First, we correlated average change in theta power separately for high and low contrast stimuli with HRV measures. In both groups, Healthy Young and Healthy 
Elderly, both conditions did not correlate with the HRV measures $(p>0.22)$. Higher change in theta power in response to high contrast scrambled images correlated with higher LF to HF power in Healthy Elderly $\left(r_{s}=0.43, p=0.044\right)$ which did not remain stable after removing three outliers $\left(r_{s}=43, p=0.07\right)$.

To investigate whether neural variability and HRV indicate changes in cognitive aging resulting from the same underlying mechanism such as prefrontal deficiency (Frewen et al., 2013; Thayer et al., 2009), we correlated the different HRV parameters with percent change in variability quenching. We expected higher variability quenching being related to higher HRV. Higher variability quenching in response to high and low contrast condition was related to lower LF to HF ratio (Contrast 10\%: average: $r_{s}=-0.54, p=$ 0.01 ; cars: $r_{s}=-0.54 ; p=0.01$, scrambled: $r=-0.56, p=0.01$; Contrast $100 \%$ : average: $r_{s}=-0.45, p=0.047 ;$ cars: $r_{s}=-0.47, p=0.037$, scrambled: $\left.r_{s}=-0.53, p=0.02\right)$ which did not remain stable after removing 4 outliers scoring 2 SD away from the mean within the group (Contrast 10\%: average: $r_{s}=-0.47, p=0.14$; cars: $r_{s}=-0.41, p=0.12$, scrambled: $r_{s}=-0.47, p=0.07$; Contrast $100 \%$ : cars: $r_{s}=-0.32, p=0.23$; scrambled: $r_{s}$ $=-0.46, p=0.07)$.

Higher variability quenching in response to low contrast condition was related to higher LF power in Healthy Young (Contrast 10\%: average: $r_{s}=0.47, p=0.037$; cars: $r_{s}=0.47$, $p=0.03$; scrambled: $r_{s}=0.52, p=0.02$ ). However, after removing 2 influential observations the correlations did not remain stable (Contrast 10\%: average: $r_{s}=-0.02, p$ $=0.94$; cars: $r_{s}=-0.001, p=0.98:$ scrambled: $\left.r=0.04, p=0.89\right)$. These results indicate that HRV in Healthy Young and Elderly is not related to variability quenching in response to high and low contrast stimuli. 


\subsection{Discussion}

Our results show that healthy individuals independent of their age show higher behavioral variability, higher P3, higher change in theta power and lower neural variability in response to a high contrast stimulus. Healthy Elderly need more time for solving standardized cognitive tests, Trail Making part A and B and Stroop Test congruent and noncongruent condition. Considering the task measurements Healthy Elderly show higher recognition time variability for cars, reduced P1 and P3 Amplitude, higher change of theta power and lower cardiac variability compared to Healthy Young. Moreover, we could show that lower cognitive performance in Healthy Elderly is related to higher behavioral, driven by low contrast condition and lower neural variability.

\subsubsection{Comparison with previous findings on intra-individual variability in recognition times}

Previous research showed that healthy older adults show increased intra-individual variability compared to young adults in tasks using simple stimuli such as circles and flickers. This effect is more pronounced in older adults, 60 plus, than for middle aged adults 40 to 60 and for choice reaction time task compared to simple RT tasks (Dykiert, Der, Starr, \& Deary, 2012). This is consistent with our results as we do not find differences in CVRT of the simple RT task but for the Stroop task and for the cars condition of our image recognition task. In Healthy Elderly worse cognitive performance was related to increased behavioral variability in low contrast condition. This goes in line with the findings that intra-individual variability of reaction times predicts age-related cognitive decline (Bielak et al., 2010; Gorus et al., 2008; MacDonald et al., 2006) and mortality (Haynes, Bauermeister, \& Bunce, 2017). Moreover, our results show that 
behavioral responses such as intra-individual variability to low contrast stimuli might be more effective to predict cognitive changes as measured by standardized tasks as the Trail Making Test in Healthy Elderly.

\subsubsection{Event Related Potentials}

We showed that Healthy Elderly are characterized by lower P1 in response to low contrast condition and lower P3 in response to high contrast condition. Lower P1 amplitude is consistent with previous findings in Healthy Elderly (Stothart et al., 2014). However, previous research shows inconsistent findings such as increased P1 amplitude in Healthy Elderly (Falkenstein, Yordanova, \& Kolev, 2006) indicating that P1 might be a less stable indicator of perceptual and cognitive changes in aging. Lower P3 amplitude characterizes elderly with cognitive decline such as $\mathrm{MCl}$ and dementia (Morrison, Rabipour, Taler, Sheppard, \& Knoefel, 2019) and in healthy middle aged compared to Healthy Young adults (Bourisly, 2016) reflecting changes in cognitive function. Consistent with previous research we could show reduced cognitive functioning as indicated by worser performance on the Trail Making Test part A and B and lower P3 amplitude in Healthy Elderly, especially in the high contrast condition. This shows that high contrast stimuli are probably more sensitive in indicating cognitive change by decrease in P3.

\subsubsection{Time-frequency bands}

Our finding of increase in theta power supports findings in PD patients with $\mathrm{MCl}(\mathrm{He}$, Zhang, Chen, Xie, Gan, Wang, et al., 2017). Increase in theta power predicts cognitive decline in PD (Cozac et al., 2016). Increase in theta power is correlated with cognitive impairment and predicts cognitive declines in PD patients (Geraedts et al., 2018) 
indicating its potential as a marker of cognitive decline. Our results did not show a relation between attention measures such as Trail Making Test and theta power. However, we could show a relation between higher theta power and lower variability performance on the Stroop Test in Healthy Elderly. This might be probably explained by differences in the montage or cognitive measures that were used.

\subsubsection{Neural variability}

Previous research reported that increased variability quenching is related to better perceptual performance in Healthy Young individuals (Arazi, Censor, et al., 2017). We did not find group differences in variability quenching between Healthy Young and Healthy Elderly. However, in response to the different contrast conditions of our task we found lower variability quenching for the low contrast condition compared to the high contrast condition in Healthy Young and Healthy Elderly. Variability quenching might increase the signal to noise ratio and thereby improve signal processing while processing the stimuli of the more difficult low contrast condition (Churchland et al., 2010). Moreover, lower neural variability correlated with lower cognitive performance in Healthy Elderly consistent with previous findings in elderly and slower performing adults (Garrett et al., 2013) as well as during maturation (McIntosh, Kovacevic, \& Itier, 2008). This might indicate that in phases of change in cognitive capacity such as maturation (Mclntosh et al., 2008) and aging increase in neural variability reflect better cognitive state. However, higher neural variability correlated with lower attention performance in Healthy Young only which can be interpreted as consistent with previous research (Arazi, Censor, et al., 2017). This might indicate that lower neural variability reflects better cognitive performance in Healthy Young. Moreover, neural variability is stable 
over time and across tasks in Healthy Young human volunteers (Arazi, Gonen-Yaacovi, et al., 2017) showing its reliability as a possible marker of cognitive changes. Nevertheless, older adults and slower performing adults had been reported to show fewer changes in brain variability (Garrett et al., 2013).

\subsubsection{Heart rate variability}

Consistent with previous research Healthy Elderly showed lower HRV compared to Healthy Young (Abhishekh et al., 2013). Previous research on the relation between cognitive functions and HRV are inconsistent and might be related to characteristics of different groups(Britton et al., 2008; Frewen et al., 2013; D. H. Kim et al., 2006; Thayer et al., 2009). HRV probably reflects sustained attention as measured by continous performance tests (Thayer et al., 2009) and memory as measured by the subtest of the MOCA (Frewen et al., 2013) in contrast to executive measures such the the Trail Making Test and the Stroop Test.

\subsubsection{Limitations and Future directions}

One limitation is the small subgroup sample size. Reliability of the analysis would benefit from increase in sample size of the Healthy Elderly and Healthy Young. Another limitation is that our groups shows contradictory result with faster recognition times in the Healthy Elderly which can not be explained by incidental buttom pressing. Recognition times were slower than $200 \mathrm{~ms}$ (Nagel et al., 2009). A possible explanation might be higher impulsivity in Healthy Elderly. Future studies might benefit by assessment of inhibitory control. Medication might be another factor which might have influenced our cardiac measurements. As certain factors such as cardioactive medication, menstrual cycle, estrogen therapy (Aronson \& Burger, 2001; von Holzen, 
Capaldo, Wilhelm, \& Stute, 2016), smoking, gender and caffeine intake (Dömötör, Szemerszky, \& Köteles, 2015; Tegegne, Man, van Roon, Riese, \& Snieder, 2018) might influence cardiac measurements future studies could asses these variables and include them in control analyses or exclude these influences in sample selection. Furthermore, other interesting marker of cognitive changes in aging might be heart evoked potential showing a heart related EEG response which had been shown to be related to arousal and attention (Luft \& Bhattacharya, 2015; Montoya, Schandry, \& Müller, 1993). Moreover, as cognitive changes might be more reflected by frontal electrodes (Saliasi, Geerligs, Lorist, \& Maurits, 2013) these should be investigated in future research probably providing more sensitive measurements of age related cognitive changes.

\subsubsection{Conclusion}

Healthy aging is characterized by cognitive, neural and cardiac changes. Our results indicate that worsening of cognitive performance might be characterized by increase in behavioral variability and lower neural variability in healthy elderly. Both, behavioral and neural variability are non-invasive, probably early markers of cognitive decline. Variability quenching is a quite novel measurement in clinical groups which needs further investigation in different age groups to prove its potential in progression of cognitive decline and probably neurodegenerative disorders such as Parkinson's disease, Lewy Body dementia and Alzheimer's disease and its suitability as a marker of clinically relevant cognitive changes. 


\section{References}

Abhishekh, H. A., Nisarga, P., Kisan, R., Meghana, A., Chandran, S., Trichur Raju, null, \& Sathyaprabha, T. N. (2013). Influence of age and gender on autonomic regulation of heart. Journal of Clinical Monitoring and Computing, 27(3), 259264. https://doi.org/10.1007/s10877-012-9424-3

Alonso, A., Huang, X., Mosley, T. H., Heiss, G., \& Chen, H. (2015). Heart rate variability and the risk of Parkinson disease: The Atherosclerosis Risk in Communities study. Annals of Neurology, 77(5), 877-883. https://doi.org/10.1002/ana.24393

Arazi, A., Censor, N., \& Dinstein, I. (2017). Neural Variability Quenching Predicts Individual Perceptual Abilities. The Journal of Neuroscience: The Official Journal of the Society for Neuroscience, 37(1), 97-109.

https://doi.org/10.1523/JNEUROSCI.1671-16.2016

Arazi, A., Gonen-Yaacovi, G., \& Dinstein, I. (2017). The Magnitude of Trial-By-Trial Neural Variability Is Reproducible over Time and across Tasks in Humans. ENeuro, 4(6). https://doi.org/10.1523/ENEURO.0292-17.2017

Arias, P., Robles-García, V., Espinosa, N., Corral, Y., \& Cudeiro, J. (2012). Validity of the finger tapping test in Parkinson's disease, elderly and young healthy subjects: is there a role for central fatigue? Clinical Neurophysiology: Official Journal of the International Federation of Clinical Neurophysiology, 123(10), 2034-2041. https://doi.org/10.1016/j.clinph.2012.04.001

Aronson, D., \& Burger, A. J. (2001). Effect of beta-blockade on heart rate variability in decompensated heart failure. International Journal of Cardiology, 79(1), 31-39.

Beck, A. T., Ward, C. H., Mendelson, M., Mock, J., \& Erbaugh, J. (1961). An inventory for measuring depression. Archives of General Psychiatry, 4, 561-571. 
Bielak, A. A., Hultsch, D. F., Strauss, E., MacDonald, S. W., \& Hunter, M. A. (2010). Intraindividual variability in reaction time predicts cognitive outcomes 5 years later. Neuropsychology, 24(6), 731.

Bourisly, A. K. (2016). Effects of aging on P300 between late young-age and early middle-age adulthood: an electroencephalogram event-related potential study. Neuroreport, 27(14), 999-1003. https://doi.org/10.1097/WNR.0000000000000644

Britton, A., Singh-Manoux, A., Hnatkova, K., Malik, M., Marmot, M. G., \& Shipley, M. (2008). The association between heart rate variability and cognitive impairment in middle-aged men and women. The Whitehall II cohort study. Neuroepidemiology, 31(2), 115-121. https://doi.org/10.1159/000148257

Caviness, J. N., Hentz, J. G., Evidente, V. G., Driver-Dunckley, E., Samanta, J., Mahant, P., ... Adler, C. H. (2007). Both early and late cognitive dysfunction affects the electroencephalogram in Parkinson's disease. Parkinsonism \& Related Disorders, 13(6), 348-354.

https://doi.org/10.1016/j.parkreldis.2007.01.003

Chapleau, M. W., \& Sabharwal, R. (2011). Methods of assessing vagus nerve activity and reflexes. Heart Failure Reviews, 16(2), 109-127. https://doi.org/10.1007/s10741-010-9174-6

Churchland, M. M., Byron, M. Y., Cunningham, J. P., Sugrue, L. P., Cohen, M. R......Shenoy, K. V. (2010). Stimulus onset quenches neural variability: a widespread cortical phenomenon. Nat Neurosci. 13(3), 369-378. https://doi:10.1038/nn.2501 
Cozac, V. V., Chaturvedi, M., Hatz, F., Meyer, A., Fuhr, P., \& Gschwandtner, U. (2016). Increase of EEG Spectral Theta Power Indicates Higher Risk of the Development of Severe Cognitive Decline in Parkinson's Disease after 3 Years. Frontiers in Aging Neuroscience, 8, 284. https://doi.org/10.3389/fnagi.2016.00284

Cummins, T. D. R., Broughton, M., \& Finnigan, S. (2008). Theta oscillations are affected by amnestic mild cognitive impairment and cognitive load. International Journal of Psychophysiology: Official Journal of the International Organization of Psychophysiology, 70(1), 75-81. https://doi.org/10.1016/j.ijpsycho.2008.06.002

Deary, I. J., \& Der, G. (2005). Reaction Time, Age, and Cognitive Ability: Longitudinal Findings from Age 16 to 63 Years in Representative Population Samples. Aging, Neuropsychology, and Cognition, 12(2), 187-215. https://doi.org/10.1080/13825580590969235

Dinstein, I., Heeger, D. J., \& Behrmann, M. (2015). Neural variability: friend or foe? Trends in Cognitive Sciences, 19(6), 322-328. https://doi.org/10.1016/j.tics.2015.04.005

Dömötör, Z., Szemerszky, R., \& Köteles, F. (2015). Subjective and objective effects of coffee consumption - caffeine or expectations? Acta Physiologica Hungarica, 102(1), 77-85. https://doi.org/10.1556/APhysiol.101.2014.012

Dykiert, D., Der, G., Starr, J. M., \& Deary, I. J. (2012). Age differences in intra-individual variability in simple and choice reaction time: systematic review and metaanalysis. PloS One, 7(10), e45759. https://doi.org/10.1371/journal.pone.0045759

Ekman, P., \& Friesen, W. V. (1976). Pictures of Facial Affect. Palo Alto.CA.Consulting Psychologists Press. 
Falkenstein, M., Yordanova, J., \& Kolev, V. (2006). Effects of aging on slowing of motorresponse generation. International Journal of Psychophysiology: Official Journal of the International Organization of Psychophysiology, 59(1), 22-29. https://doi.org/10.1016/j.ijpsycho.2005.08.004

Feuerriegel, D., Churches, O., Hofmann, J., \& Keage, H. A. D. (2015). The N170 and face perception in psychiatric and neurological disorders: A systematic review. Clinical Neurophysiology: Official Journal of the International Federation of Clinical Neurophysiology, 126(6), 1141-1158. https://doi.org/10.1016/j.clinph.2014.09.015

Finnigan, S., \& Robertson, I. H. (2011). Resting EEG theta power correlates with cognitive performance in healthy older adults. Psychophysiology, 48(8), 10831087. https://doi.org/10.1111/j.1469-8986.2010.01173.x

Folstein, M. F., Folstein, S. E., \& McHugh, P. R. (1975). "Mini-mental state". A practical method for grading the cognitive state of patients for the clinician. Journal of Psychiatric Research, 12(3), 189-198.

Frewen, J., Finucane, C., Savva, G. M., Boyle, G., Coen, R. F., \& Kenny, R. A. (2013). Cognitive function is associated with impaired heart rate variability in ageing adults: the Irish longitudinal study on ageing wave one results. Clinical Autonomic Research: Official Journal of the Clinical Autonomic Research Society, 23(6), 313-323. https://doi.org/10.1007/s10286-013-0214-x

Garrett, D. D., Kovacevic, N., McIntosh, A. R., \& Grady, C. L. (2013). The modulation of BOLD variability between cognitive states varies by age and processing speed. Cerebral Cortex (New York, N.Y.: 1991), 23(3), 684-693. https://doi.org/10.1093/cercor/bhs055 
Geraedts, V. J., Boon, L. I., Marinus, J., Gouw, A. A., van Hilten, J. J., Stam, C. J., .. Contarino, M. F. (2018). Clinical correlates of quantitative EEG in Parkinson disease: A systematic review. Neurology, 91(19), 871-883. https://doi.org/10.1212/WNL.0000000000006473

Givens, B. (1996). Stimulus-evoked resetting of the dentate theta rhythm: relation to working memory. Neuroreport, 8(1), 159-163.

Gorus, E., De Raedt, R., Lambert, M., Lemper, J.-C., \& Mets, T. (2008). Reaction times and performance variability in normal aging, mild cognitive impairment, and Alzheimer's disease. Journal of Geriatric Psychiatry and Neurology, 21(3), 204218. https://doi.org/10.1177/0891988708320973

Guillaume, C., Guillery-Girard, B., Chaby, L., Lebreton, K., Hugueville, L., Eustache, F., Fiori, N., 2009. The time course of repetition effects for familiar faces and objects: an ERP study. Brain Res. 1248, 149-161. https://doi.org/10.1016/j.brainres.2008.10.069

Harper, J., Malone, S. M., \& lacono, W. G. (2017). Theta- and delta-band EEG network dynamics during a novelty oddball task. Psychophysiology, 54(11), 1590-1605. https://doi.org/10.1111/psyp.12906

Haynes, B. I., Bauermeister, S., \& Bunce, D. (2017). A Systematic Review of Longitudinal Associations Between Reaction Time Intraindividual Variability and Age-Related Cognitive Decline or Impairment, Dementia, and Mortality. Journal of the International Neuropsychological Society: JINS, 23(5), 431-445. https://doi.org/10.1017/S1355617717000236

He, X., Zhang, Y., Chen, J., Xie, C., Gan, R., Wang, L., \& Wang, L. (2017). Changes in theta activities in the left posterior temporal region, left occipital region and right 
frontal region related to mild cognitive impairment in Parkinson's disease patients. The International Journal of Neuroscience, 127(1), 66-72. https://doi.org/10.3109/00207454.2016.1143823

He, X., Zhang, Y., Chen, J., Xie, C., Gan, R., Yang, R., ... Wang, L. (2017). The patterns of EEG changes in early-onset Parkinson's disease patients. The International Journal of Neuroscience, 127(11), 1028-1035.

https://doi.org/10.1080/00207454.2017.1304393

Hedden, T., \& Gabrieli, J. D. E. (2004). Insights into the ageing mind: a view from cognitive neuroscience. Nature Reviews. Neuroscience, 5(2), 87-96. https://doi.org/10.1038/nrn1323

Hsieh, S., \& Lin, Y.-C. (2017). Stopping ability in younger and older adults: Behavioral and event-related potential. Cognitive, Affective \& Behavioral Neuroscience, 17(2), 348-363. https://doi.org/10.3758/s13415-016-0483-7

Jensen, B. H., Hougaard, A., Amin, F. M., Larsson, H. B. W., \& Ashina, M. (2015). Structural asymmetry of cortical visual areas is related to ocular dominance. Neuroreport, 26(17), 1071-1076. https://doi.org/10.1097/WNR.0000000000000470 Jonas, J., Jacques, C., Liu-Shuang, J., Brissart, H., Colnat-Coulbois, S., Maillard, L., \& Rossion, B. (2016). A face-selective ventral occipito-temporal map of the human brain with intracerebral potentials. Proceedings of the National Academy of Sciences of the United States of America, 113(28), E4088-4097. https://doi.org/10.1073/pnas.1522033113

Kessler, R. C., Birnbaum, H., Bromet, E., Hwang, I., Sampson, N., \& Shahly, V. (2010). Age differences in major depression: results from the National Comorbidity 
Survey Replication (NCS-R). Psychological Medicine, 40(2), 225-237. https://doi.org/10.1017/S0033291709990213

Kim, D. H., Lipsitz, L. A., Ferrucci, L., Varadhan, R., Guralnik, J. M., Carlson, M. C., ... Chaves, P. H. M. (2006). Association between reduced heart rate variability and cognitive impairment in older disabled women in the community: Women's Health and Aging Study I. Journal of the American Geriatrics Society, 54(11), 17511757. https://doi.org/10.1111/j.1532-5415.2006.00940.x

Kim, M., Lee, T. H., Kim, J.-H., Hong, H., Lee, T. Y., Lee, Y., ... Kwon, J. S. (2018). Decomposing P300 into correlates of genetic risk and current symptoms in schizophrenia: An inter-trial variability analysis. Schizophrenia Research, 192, 232-239. https://doi.org/10.1016/j.schres.2017.04.001

Latreille, V., Carrier, J., Gaudet-Fex, B., Rodrigues-Brazète, J., Panisset, M., Chouinard, S., ... Gagnon, J.-F. (2016). Electroencephalographic prodromal markers of dementia across conscious states in Parkinson's disease. Brain: $A$ Journal of Neurology, 139(Pt 4), 1189-1199.

https://doi.org/10.1093/brain/aww018

Li, S. C., Huxhold, O., \& Schmiedek, F. (2004). Aging and attenuated processing robustness. Evidence from cognitive and sensorimotor functioning. Gerontology, 50(1), 28-34. https://doi.org/10.1159/000074386

Lithfous, S., Tromp, D., Dufour, A., Pebayle, T., Goutagny, R., \& Després, O. (2015). Decreased theta power at encoding and cognitive mapping deficits in elderly individuals during a spatial memory task. Neurobiology of Aging, 36(10), 28212829. https://doi.org/10.1016/j.neurobiolaging.2015.07.007 
López, M. E., Cuesta, P., Garcés, P., Castellanos, P. N., Aurtenetxe, S., Bajo, R., ... Fernandez, A. (2014). MEG spectral analysis in subtypes of mild cognitive impairment. Age (Dordrecht, Netherlands), 36(3), 9624. https://doi.org/10.1007/s11357-014-9624-5

Lubitz, A. F., Niedeggen, M., \& Feser, M. (2017). Aging and working memory performance: Electrophysiological correlates of high and low performing elderly. Neuropsychologia, 106, 42-51. https://doi.org/10.1016/j.neuropsychologia.2017.09.002

Luft, C. D. B., \& Bhattacharya, J. (2015). Aroused with heart: Modulation of heartbeat evoked potential by arousal induction and its oscillatory correlates. Scientific Reports, 5. https://doi.org/10.1038/srep15717

Luque-Casado, A., Perales, J. C., Cárdenas, D., \& Sanabria, D. (2016). Heart rate variability and cognitive processing: The autonomic response to task demands. Biological Psychology, 113, 83-90. https://doi.org/10.1016/j.biopsycho.2015.11.013

MacDonald, S. W., Nyberg, L., \& Bäckman, L. (2006). Intra-individual variability in behavior: links to brain structure, neurotransmission and neuronal activity. Trends in Neurosciences, 29(8), 474-480.

Macdonald, S. W. S., Hultsch, D. F., \& Dixon, R. A. (2008). Predicting impending death: inconsistency in speed is a selective and early marker. Psychology and Aging, 23(3), 595-607. https://doi.org/10.1037/0882-7974.23.3.595

MacDonald, S. W. S., Li, S.-C., \& Bäckman, L. (2009). Neural underpinnings of withinperson variability in cognitive functioning. Psychology and Aging, 24(4), 792-808. https://doi.org/10.1037/a0017798 
Malik, M., Cripps, T., Farrell, T., \& Camm, A. J. (1989). Prognostic value of heart rate variability after myocardial infarction. A comparison of different data-processing methods. Medical and Biological Engineering and Computing, 27(6), 603. https://doi.org/10.1007/BF02441642

Mazaheri, A., Segaert, K., Olichney, J., Yang, J.-C., Niu, Y.-Q., Shapiro, K., \& Bowman, H. (2018). EEG oscillations during word processing predict $\mathrm{MCl}$ conversion to Alzheimer's disease. Neurolmage: Clinical, 17, 188-197. https://doi.org/10.1016/j.nicl.2017.10.009

McIntosh, A.R., Kovacevic, N. \& Itier, R.J. (2008). Increased Brain Signal Variability Accompanies Lower Behavioral Variability in Development. PLOS Computational Biology, 4(7): e 1000106. https://doi:10.1371/journal.pcbi.10000106

Miloserdov, K., Schmidt-Samoa, C., Williams, K., Weinrich, C., Bürk, K., Trenkwalder, C., ... Wilke, M. (2019). Misperceptions and Intra-Individual Variability in Parkinson's disease: a behavioral and fMRI study. Manuscript submitted for publication.

Montoya, P., Schandry, R., \& Müller, A. (1993). Heartbeat evoked potentials (HEP): topography and influence of cardiac awareness and focus of attention. Electroencephalography and Clinical Neurophysiology, 88(3), 163-172.

Morrison, C., Rabipour, S., Taler, V., Sheppard, C., \& Knoefel, F. (2019). Visual EventRelated Potentials in Mild Cognitive Impairment and Alzheimer's Disease: A Literature Review. Current Alzheimer Research, 16(1), 67-89. https://doi.org/10.2174/1567205015666181022101036 
Musaeus, C. S., Engedal, K., Høgh, P., Jelic, V., Mørup, M., Naik, M., ... Andersen, B. B. (2018). EEG Theta Power Is an Early Marker of Cognitive Decline in Dementia due to Alzheimer's Disease. Journal of Alzheimer's Disease: JAD, 64(4), 13591371. https://doi.org/10.3233/JAD-180300

Nagel, I. E., Preuschhof, C., Li, S.-C., Nyberg, L., Bäckman, L., Lindenberger, U., \& Heekeren, H. R. (2009). Performance level modulates adult age differences in brain activation during spatial working memory. Proceedings of the National Academy of Sciences of the United States of America, 106(52), 22552-22557. https://doi.org/10.1073/pnas.0908238106

Oostenveld, R., Fries, P., Maris, E., \& Schoffelen, J.-M. (2011). FieldTrip: Open source software for advanced analysis of MEG, EEG, and invasive electrophysiological data. Computational Intelligence and Neuroscience, 2011, 156869. https://doi.org/10.1155/2011/156869

Papenberg, G., Bäckman, L., Chicherio, C., Nagel, I. E., Heekeren, H. R., Lindenberger, U., \& Li, S.-C. (2011). Higher intraindividual variability is associated with more forgetting and dedifferentiated memory functions in old age. Neuropsychologia, 49(7), 1879-1888. https://doi.org/10.1016/j.neuropsychologia.2011.03.013

Porges, S. W. (2007). A phylogenetic journey through the vague and ambiguous Xth cranial nerve: a commentary on contemporary heart rate variability research. Biological Psychology, 74(2), 301-307. https://doi.org/10.1016/j.biopsycho.2006.08.007

Reitan, R.M., \& Wolfson, D. (1993). The Halstead-Reitan Neuropsychological Test Battery: Theory and Clinical Interpretation 2nd edition. Neuropsychology Ress, Tuscon. 
Saavedra, C., Olivares, E. I., \& Iglesias, J. (2012). Cognitive decline effects at an early stage: evidence from N170 and VPP. Neuroscience Letters, 518(2), 149-153. https://doi.org/10.1016/j.neulet.2012.04.075

Saliasi, E., Geerligs, L., Lorist, M. M., \& Maurits, N. M. (2013). The relationship between P3 amplitude and working memory performance differs in young and older adults. PloS One, 8(5), e63701. https://doi.org/10.1371/journal.pone.0063701

Salti, M., Bar-Haim, Y., \& Lamy, D. (2012). The P3 component of the ERP reflects conscious perception, not confidence. Consciousness and Cognition, 21(2), 961968. https://doi.org/10.1016/j.concog.2012.01.012

Shaffer, F., McCraty, R., \& Zerr, C. L. (2014). A healthy heart is not a metronome: an integrative review of the heart's anatomy and heart rate variability. Frontiers in Psychology, 5, 1040. https://doi.org/10.3389/fpsyg.2014.01040

Shine, J. M., Halliday, G. H., Carlos, M., Naismith, S. L., \& Lewis, S. J. G. (2012). Investigating visual misperceptions in Parkinson's disease: a novel behavioral paradigm. Movement Disorders: Official Journal of the Movement Disorder Society, 27(4), 500-505. https://doi.org/10.1002/mds.24900

Speer, M. E., \& Soldan, A. (2015). Cognitive reserve modulates ERPs associated with verbal working memory in healthy younger and older adults. Neurobiology of Aging, 36(3), 1424-1434. https://doi.org/10.1016/j.neurobiolaging.2014.12.025

Stothart, G., Tales, A., Hedge, C., \& Kazanina, N. (2014). Double peaked P1 visual evoked potentials in healthy ageing. Clinical Neurophysiology: Official Journal of the International Federation of Clinical Neurophysiology, 125(7), 1471-1478. https://doi.org/10.1016/j.clinph.2013.11.029 
Stroop, J. R. (1935). Studies of interference in serial verbal reactions. Journal of Experimental Psychology, 18(6), 643-662. https://doi.org/10.1037/h0054651

Tarvainen, M. P., Niskanen, J.-P., Lipponen, J. A., Ranta-Aho, P. O., \& Karjalainen, P. A. (2014). Kubios HRV--heart rate variability analysis software. Computer Methods and Programs in Biomedicine, 113(1), 210-220. https://doi.org/10.1016/j.cmpb.2013.07.024

Tegegne, B. S., Man, T., van Roon, A. M., Riese, H., \& Snieder, H. (2018). Determinants of heart rate variability in the general population: The Lifelines Cohort Study. Heart Rhythm, 15(10), 1552-1558. https://doi.org/10.1016/j.hrthm.2018.05.006

Thayer, J. F., Hansen, A. L., Saus-Rose, E., \& Johnsen, B. H. (2009). Heart rate variability, prefrontal neural function, and cognitive performance: the neurovisceral integration perspective on self-regulation, adaptation, and health. Annals of Behavioral Medicine: A Publication of the Society of Behavioral Medicine, 37(2), 141-153. https://doi.org/10.1007/s12160-009-9101-z Troyer, A. K., Moscovitch, M., \& Winocur, G. (1997). Clustering and switching as two components of verbal fluency: evidence from younger and older healthy adults. Neuropsychology, 11(1), 138-146.

Vertes, R. P. (2005). Hippocampal theta rhythm: a tag for short-term memory. Hippocampus, 15(7), 923-935. https://doi.org/10.1002/hipo.20118 von Holzen, J. J., Capaldo, G., Wilhelm, M., \& Stute, P. (2016). Impact of endo- and exogenous estrogens on heart rate variability in women: a review. Climacteric: The Journal of the International Menopause Society, 19(3), 222-228. https://doi.org/10.3109/13697137.2016.1145206 
Wilimzig, C., Ragert, P., \& Dinse, H. R. (2012). Cortical topography of intracortical inhibition influences the speed of decision making. Proceedings of the National Academy of Sciences of the United States of America, 109(8), 3107-3112. https://doi.org/10.1073/pnas.1114250109

Wykowska, A., \& Schubö, A. (2012). Action intentions modulate allocation of visual attention: electrophysiological evidence. Frontiers in Psychology, 3, 379. https://doi.org/10.3389/fpsyg.2012.00379

Yan, T., Bi, X., Zhang, M., Wang, W., Yao, Z., Yang, W., \& Wu, J. (2016). Age-related oscillatory theta modulation of multisensory integration in frontocentral regions. Neuroreport, 27(11), 796-801. https://doi.org/10.1097/WNR.0000000000000609

\section{Acknowledgements}

We thank Severin Heumüller for excellent computer and programming support.

\section{Author Contributions}

(1) Research Project: A. Conception, B. Organization, C. Execution, Supervision; (2) Statistical Analysis: A. Design, B. Execution, C. Review and Critique; (3) EEG Analysis: A. Design, B. Execution, C. Review and Critique; (4) ECG Anaysis: A. Design, B. Execution, C. Review and Critique (5) Manuscript Preparation: A. Writing of the First Draft, B. Review and Critique.

K.M.: 1A, 1B, 1C, 2A, 2B, 3A, 4A, 4B, 4C, 5A, 5B C.S.: 1A, 2A, 2B, 2C; DT: 1B, 1C, 3A, 3B, 3C; IS.: 3A, 3C, 4A, 4C; A.S.: 1A, 3A, 3C; M.W.: 1A, 1C, 2A, 2C, 3A, 3C, 4A, 4C. 


\section{Funding}

This work was supported by the Herman and Lilly Schilling Foundation (MW), the DFG Center for Nanoscale Microscopy \& Molecular Physiology of the Brain (CNMPB) and the Leibniz-Science Campus Primate Cognition.

\section{Competing Interests}

None.

\section{Ethics approval}

Permission for the study was obtained from the medical ethics committees of the University Medical Center Goettingen, Germany.

\section{Data sharing statement}

We will share all data and materials upon request. 
Supplementary Materials

Behavioral, Neural and Heart Rate Variability and their Relation to Cognitive Performance in Healthy Young and Healthy Elderly

Miloserdov et al., in preparation

Supplementary Materials contains:

2 Supplementary Figures 


\section{Supplementary Figures}

A

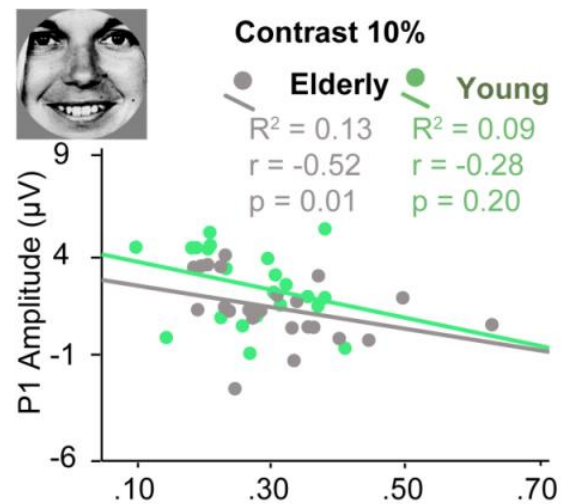

Contrast $100 \%$

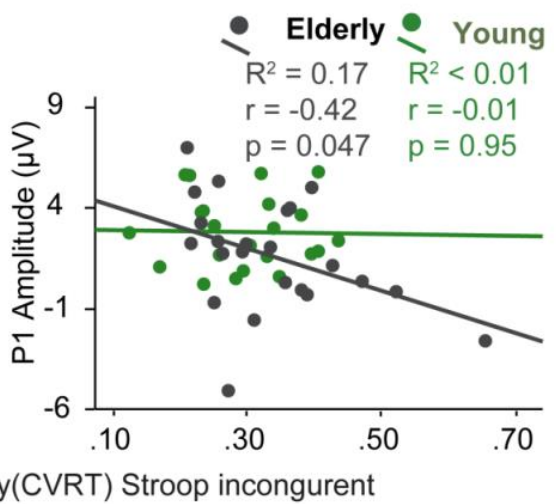

B

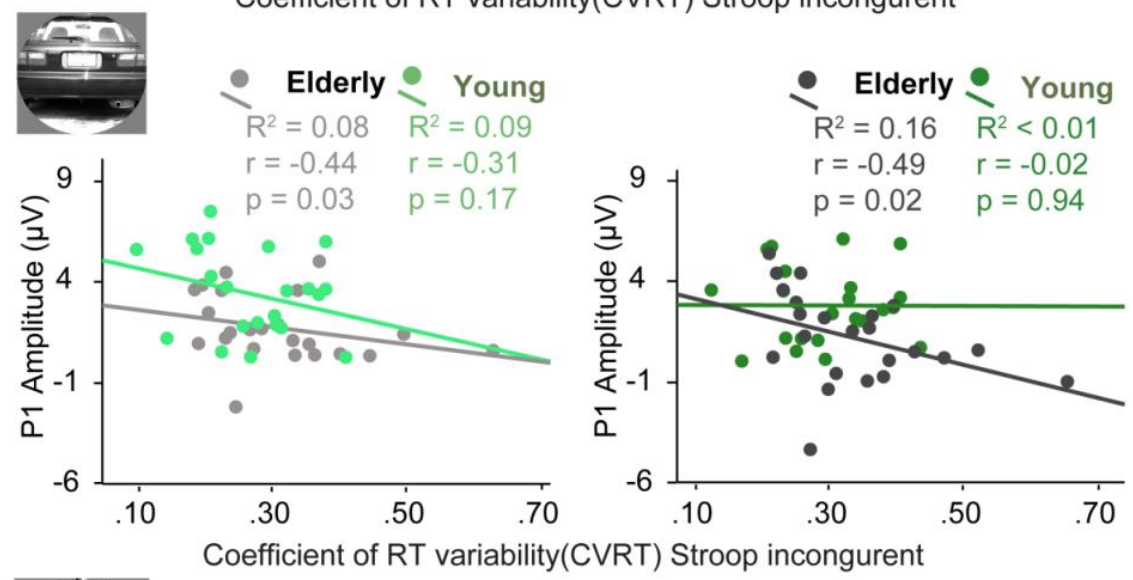

C
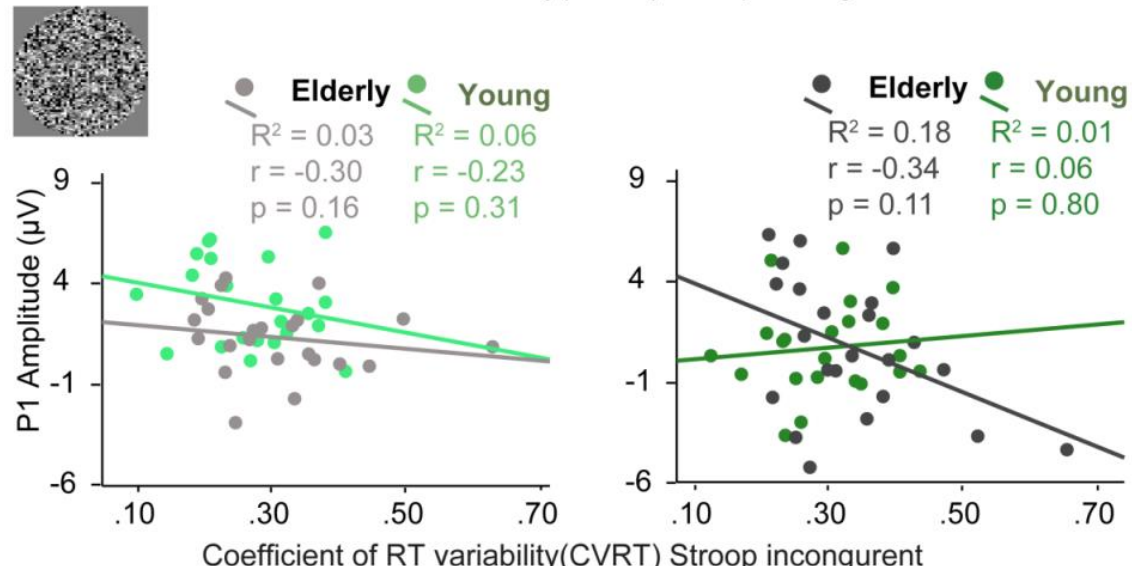

Supplementary Figure S3.1: Association of P1 Amplitude and coefficient of reaction time variability of incongruent condition of the Stroop task. Correlation between P1 amplitude for A) faces B) cars and C) scrambled images left Contrast $10 \%$ and right Contrast $100 \%$ displayed on the $y$-axis and coefficient of reaction time variability of the Stroop task on the x-axis. Green dots show the individual values of Healthy Young volunteers $(\mathrm{N}=22)$ and grey dots the individual values of Healthy Elderly volunteers $(N=24)$. 

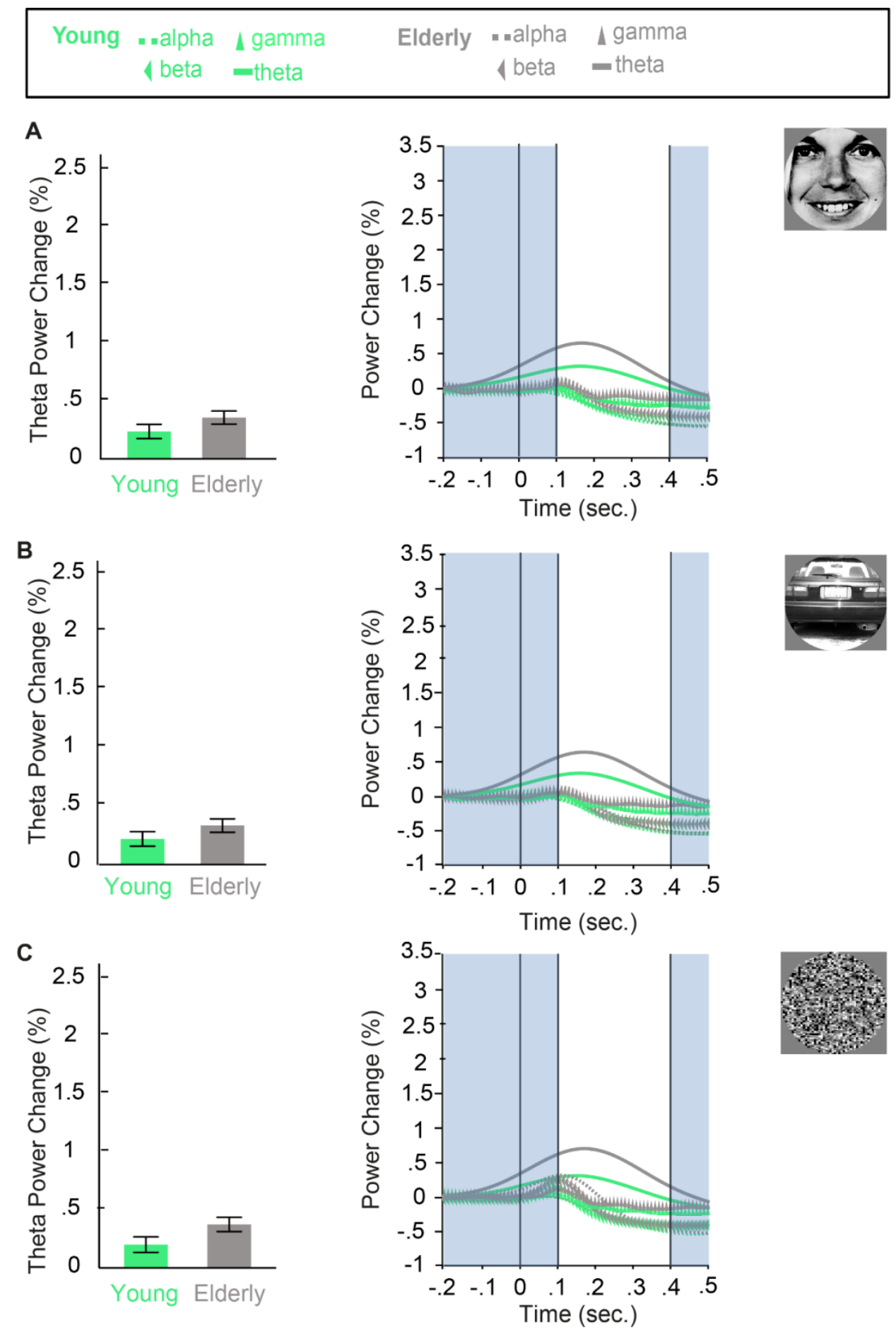

Supplementary Figure S3.2: Theta power of the low contrast condition. Average percentage of Theta power change, separated by stimulus type, A) face or B) car, and C) scrambled image and by subject group, Healthy Young $(N=22)$ in green and Healthy Elderly $(N=24)$ in gray. On the left in all panels, means of the theta power change across subjects in each group are shown. Error bars denote 
S.E.M. across subjects. In the center power change in different frequency bands of interest such as alpha, beta, gamma and theta are shown. * denotes a significant difference, $p<0.05$ (black star as assessed by the ANOVA and post-hoc tests) ${ }^{* *}$ denotes a significant difference, $p<0.005$ (black star as assessed by the ANOVA and post-hoc tests).

A

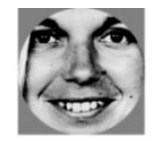

Contrast $10 \%$

Elderly $\bigcirc$ Young

$R^{2}=0.02 \quad R^{2}=0.18$

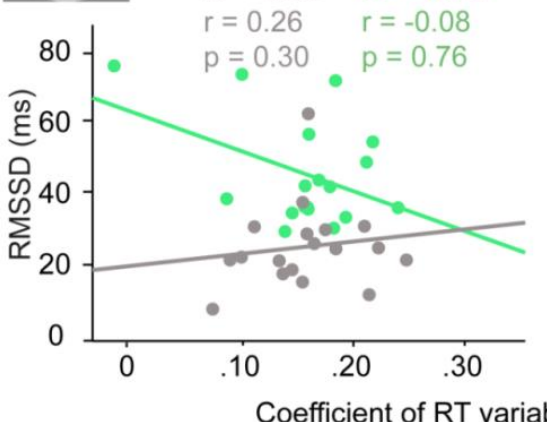

Coefficient of RT variability (CVRT) faces (\%)

B

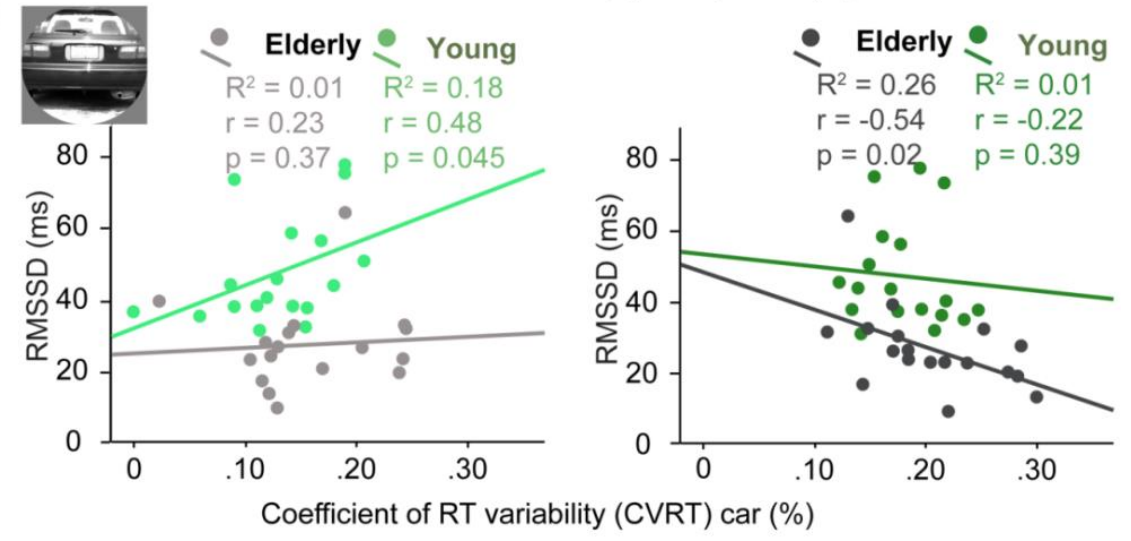

C

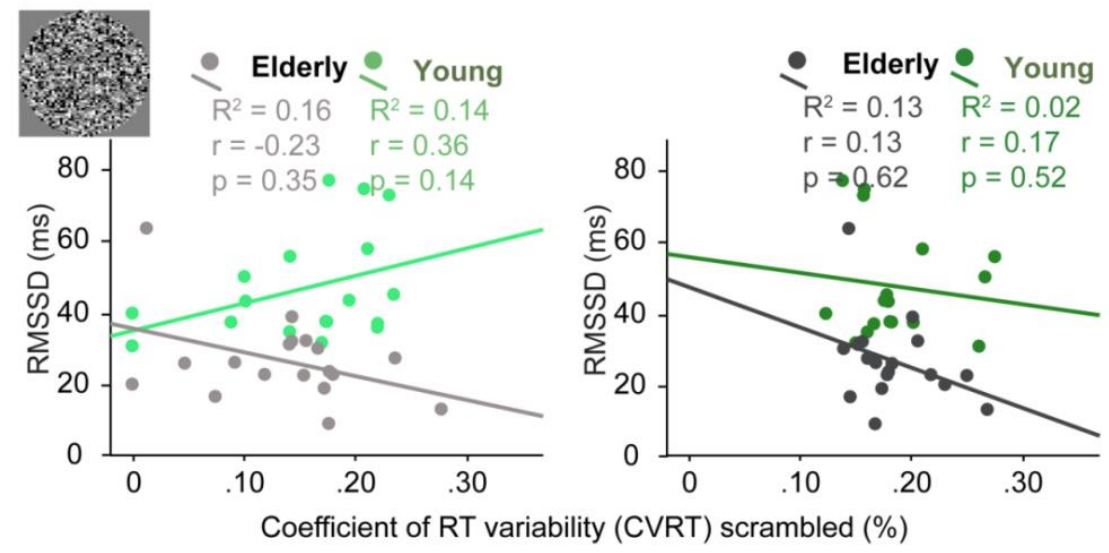

Supplementary Figure S3.3: Relation of Heart Rate Variability (RMSSD) during the task and behavioral variability. Correlation between intra-individual variability of A) faces B) cars and C) scrambled images left Contrast $10 \%$ and right Contrast $100 \%$ displayed on the $y$-axis and RMSSD on the 
$x$-axis. Green dots show the individual values of Healthy Young volunteers $(\mathrm{N}=18$ (without outlier)) and grey dots the individual values of Healthy Elderly volunteers $(\mathrm{N}=18$ (without outlier)). 


\section{Chapter 4}

\section{General Discussion}

This thesis examined objective and quatifiable measures of visual misperceptions and behavioral variability in Parkinson's disease. 1)Differences between hallucinating and non-hallucinating PD patients in perceptual error score and behavioral variability and the relation to reduced functional connectivity between attentional networks and perceptual errors and reduced functional connectivity between somatomotor and fronto-parietal network and behavioral variability are discussed from the perspective of behavioral and

neural models. 2) Differences in P1, P3 amplitude, theta power, HRV between Healthy Young and Healthy Elderly and the relation of behavioral and neural variability to cognitive measures in Healthy Elderly are discussed from the perspective of different behavioral, cognitive, neural and cardiac models. The major topic of this thesis focused on the discussion of the potential of different objective measures such as psychophysical performance, variability quenching and fuctional connectivity as possible marker of different non-motor symptoms phenotypes in PD as well as potential diagnostic implications.

\subsection{Non-motor symptoms in Parkinson's disease}

Non-motor symptoms in PD can be described in terms of impairments which can be measured by psychophysical performance. Cognitive symptoms can be categorized in different domains such as memory deficits, attentional deficits, executive dysfunction and visuoperceptual deficits. To measure memory deficits, recognition and semantic 
memory tasks can be used. Attention deficits can be tested with tasks measuring executive control, orienting, arousal and cognitive fluctuations. Tasks measuring executive dysfunctions include learning from feedback and cognitive flexibility such as rule-shifting, working memory and response inhibition (Gratwicke, Jahanshahi, \& Foltynie, 2015). Dysfunctions of the different cognitive domains are present in neuropathologies such as attentional fluctuations in ADHD, response disinhibition in ICB, attentional deficits and executive dysfunction in $\mathrm{MCl}$, memory and $\mathrm{VH}$ in $\mathrm{LB}$ dementia and $\mathrm{VH}$ in schizophrenia but some can also characterize physiological changes during the life-span such as age related decline in response inhibition, selective and divided attention (Geerligs, Saliasi, Maurits, Renken, \& Lorist, 2014; Harada, Natelson Love, \& Triebel, 2013; Haring et al., 2013). Some processes of the different cognitive domains such as increased behavioral variability may be general or shared in healthy aging, ADHD, MCl, PD, schizophrenia, LB dementia and PD (Dykiert et al., 2012; MacDonald et al., 2006; Rentrop et al., 2010; van Belle et al., 2015) while others might be more specific such as VH occuring in PD, schizophrenia and LB dementia (Shine, O'Callaghan, Halliday, \& Lewis, 2014). Objective behavioral measures, which are sensitive to different non-motor profiles such as cognitive decline and $\mathrm{VH}$ might be used for differential diagnostics.

\subsection{Behavioral, neural and cardiac variability in healthy aging}

Intra-individual variability of reaction times is a sensitive predictor for age related cognitive decline (Bielak et al., 2010; Gorus et al., 2008; MacDonald et al., 2006). Behavioral variability increases in healthy aging (Dykiert et al., 2012) and is predictive for transition from healthy aging to mild cognitive impairments (Cherbuin, Sachdev, \& 
Anstey, 2010). The correlation of higher behavioral variability with worse performance on the Trail Making Test in healthy elderly is consistent with previous research (Bielak et al., 2010; Gorus et al., 2008; MacDonald et al., 2006). We also tested another candidate of lower cognitive functioning, HRV in healthy elderly (Frewen et al., 2013; Thayer et al., 2009). In our group of healthy elderly we did not find a relation between lower HRV and cognitive decline. This is probably explained by measurements of different cognitive functions. Previous research showed a relation between HRV and sustained attention by continous performance tests (Thayer et al., 2009) and between memory recall, language subtest of the MOCA (Frewen et al., 2013) which are probably more sensitive in detecting HRV and age-related cognitive changes than the Trail Making Test and the Stroop Test. Another novel candidate of cognitive decline in healthy aging is neural variability. To our knowledge age related differences in variability quenching and their relation to cognitive functioning, behavioral variability and cardiac variability were not investigated before. Our data shows that worse cognitive performance is related to lower neural variability in healthy eldery and higher neural variability in healthy young which might be related to differences or changes in cognitive capacity. In contrast to our expectations based on previous research showing that neural variability is related to perceptual performance (Arazi, Censor, et al., 2017; Schurger, Sarigiannidis, Naccache, Sitt, \& Dehaene, 2015) we did not find a consistent correlation between neural variability and behavioral variability. Possible explanations might be that the task was not complex enough for the subtle physiological age related changes. Larger behavioral variability mgiht be provoked by more involvement of executive functions and need more complex tasks (Dixon et al., 2007; West, Murphy, Armilio, Craik, \& Stuss, 2002). However, it is also possible that the task was not simple enough to show age related differences in 
behavioral variability given that differences in behavioral variability are lower for cognitively more demanding task (Hultsch, MacDonald, \& Dixon, 2002). In case that task complexity is driving the effect it would be reasonable to see differences between the high contrast condition, being easier to recognize, and low contrast condition which is more difficult to recognize. Exploratory analyses, using a separate ANOVA for the low and high contrast condition showed that healthy elderly quenched less for the window 100 to 200 ms after stimulus presentation in the low contrast condition indicating that neural variability differentiates between higher and lower performing group in perceptually more demanding tasks where healthy young probably improve signal processing with increasing the signal to noise ratio by increased variability quenching (Churchland, 2010). However, another explanation might also be that the tasks do not differ in respect to perceptual demands but in cognitive demands such as shifting or dividing attention. Probably a task using a paradigm with additional difficulty such as distractors might result in higher behavioral variability which is related to neural variability in healthy elderly. Nevertheless, behavioral and neural variability seem to reflect general executive functioning in healthy adults and are possible marker of decline in executive functions, probably more effective in perceptually demanding conditions.

\subsection{Behavioral variability in Parkinson's disease}

Behavioral variability is assumed to be sensitive to already detect subtle decline in congnition (MacDonald et al., 2006). Increased behavioral variability had been shown to be associated with age related cognitive decline (Lövdén, Li, Shing, \& Lindenberger, 2007; MacDonald, Hultsch, \& Dixon, 2003) higher risk for development of mild cognitive impairment (Bielak et al., 2010; Cherbuin et al., 2010; Kälin et al., 2014) and predicted 
transition from mild cognitive impairment to dementia (Gorus et al., 2008; Tales et al., 2012). In prodromal Huntington Disease (Musso et al., 2015) and in untreated PD patients behavioral variability was increased (Burton et al., 2006; Camicioli et al., 2008). This clearly indicates potential of behavioral variability as an early marker of cognitive impairments in neurodegenerative disorders (Costa et al., 2019). Interestingly, behavioral variability increased over time in PD patients with mild cognitive impairment and dementia compared to PD patients without cognitive decline (de Frias et al., 2012). Increased behavioral variability in our sample of hallucinating PD patients indicates higher chances for development of cognitive decline in PD-VH (Diederich et al., 2009). Given that increased behavioral variability was also shown in another clinical groups such ADHD, OCD and ICD who do not experience VH but attentional and inhibitory deficits (Abramovitch et al., 2019; Kertzman et al., 2018; Kofler et al., 2013) showing that increased behavioral variability is driven by the same deficits in attentional and executive function. Thus, objectively task derived intra-individual variability, especially from more complex tasks, is a promising marker of attentional and executive dysfunction in different neurological disorders.

\subsection{Visual Misperceptions and Hallucinations in Parkinson's disease}

$\mathrm{VH}$ are related to non-motor symptoms of different domains such as depression (Holroyd, Currie, \& Wooten, 2001), sleep (Sanchez-Ramos et al., 1996) and cognitive disturbances (Sanchez-Ramos et al., 1996). Interestingly, especially decline in executive function but not episodic memory is related to experience of VH in PD (Creese et al., 2018). This indicates that executive dysfunction is common to dementia and VH in PD but memory deficits are specific for dementia. Execuitve dysfunction consists of different 
functions such as rule-shifting, response inhibition and working memory (Gratwicke et al., 2015). The possible contribution of these different executive functions in explaining $\mathrm{VH}$ is indicated by increased perceptual errors and behavioral fluctuation of PD-VH as shown by our group and increase in percpetual errors and mental imagery in PD-VH (Shine, Keogh, et al., 2015). Attentional and inhibitory control of perceptual errors might be indicated by network related measurements which were associated with different congitive functions (Prell, 2018; Shine, Keogh, et al., 2015). As increased perceptual error score in PD-VH was shown to be consistent. It seems to be an objective measure to detect those with an increased risk to experience $\mathrm{VH}$, probably even et al early $\mathrm{PD}$ stage (Kulisevsky \& Pagonabarraga, 2009). Nevertheless, additional new neural measurements such as functional connectivity of Resting state networks might provide additional insights into neural and functional dysfunctions related to different symptom profiles and have the advantage of not being influenced by expectations or ideas about the desirable performance.

\subsection{Resting state networks in Parkinson's disease}

Most literature on Resting state fMRI in PD investigated connectivity of motor regions, icnluding the somatomotor network (Hohenfeld, Werner, \& Reetz, 2018). However, by

now findings are inconsistent on hypo- and hyperconnectivity of the motor regions (Hohenfeld et al., 2018). Moreover, also the default mode network was of main interest showing no or only a few differences in DMN connectivity pattern (Hohenfeld et al., 2018) such as hyperconnectivity of the DMN in PD (Campbell et al., 2015; Gorges et al., 2015). 


\subsubsection{Visual misperceptions in Parkinson's disease}

In PD reporting $\mathrm{VH}$ increased connectivity within the DMN had been shown (Franciotti et al., 2015; Shine, Keogh, et al., 2015; Yao et al., 2015, 2014). In addition, (Shine, Keogh, et al., 2015) hyperconnectivity of the DMN was not only related to perceptual errors but also to mental imagery. As we did not measure strength of mental imagery in our sample it might be possible that our sample differs with respect to mental imagery to other PD$\mathrm{VH}$ and thereby do not show hyperconnectivity of the DMN. Moreover, as alterations in DMN are inconsistent other sample characteristics such as differences in respect to motor- and non-motor symtpoms might also be considered in explaining the missing DMN hyperactivitiy of our sample. Nevertheless, consistent with previous research we found that increase in visual misperceptions is related to hypoconnectivity between dorsal attention network and the salience network (Shine, Keogh, et al., 2015). This provides support for involvement of attentional and executive dysfunction in VH in PD (Shine, Keogh, et al., 2015). Previous imaging studies had shown that connectivity between the DAN and the insula and anterior cingulate, which are part of the salience network, is reduced in PD patients with $\mathrm{MCl}$ (Baggio et al., 2015). Moreover, impulse control symptoms were shown to be related to hyperconnectivity within the salience netowork (Tessitore et al., 2017). Increased connectivity of the salience network was even present in unmedicated PD patients who developed impulse control behaviors during disease progression (Tessitore et al., 2016). This indicates that networks involved in executive control might be involved in hallucinatory experience. Given that the hypoconnectivity between DAN and salience network might be not unique to $\mathrm{VH}$ in PD combing Resting state function connectivity with other objective measures such as 
perceptual errors might be more reliable in characterizing PD subgroup at risk for visual misperceptions and or $\mathrm{VH}$.

\subsubsection{Behavioral variability}

Previous research on behavioral variability in healthy adults showed that increased behavioral variability was related to increased activation of middle frontal areas, right inferior parietal and thalamic regions (Bellgrove, Hester, \& Garavan, 2004). In healthy older adults lower performance variability was related to in creased activity of the inferior parietal cortex (Cabeza \& Nyberg, 2000; Shannon \& Buckner, 2004). Given that frontoparietal regions are important for inhibitory control higher task-related recrutment of these regions reflects increased need for inhibitory control in healthy indivduals with higher behavioral variability (MacDonald et al., 2009). Moreover, lower behavioral variability was related to stronger negative correlation between activity of the DMN and DAN (Kelly, Uddin, Biswal, Castellanos, \& Milham, 2008) indicating that behavioral variabiltiy reflects functional coordination between different networks such as reduced DMN and increased DAN (MacDonald et al., 2009). Moreover, in a neuropsychiatric disorder of attention and inhibitory control, e.g. ADHD, lower behavioral variability was related to increased activity of supplementary pre-motor area, cerebellum and inferior parietal lobule and increased behavioral variability was related to higher activity of the prefrontal cortex (Simmonds et al., 2007) indicating that coordination of prefrontal, parietal and premotor areas and reduced connectivity might contribute to behavioral variability. The deficits in attention and inhibitory control seem to be reflected in our findings of increased behavioral variability in PD patients being related to hypoconnectiviy of SMN and right fronto-parietal network. Changes in sensorimotor-, 
frontal- and parietal regions were also related with other non-motor symptoms in PD. PD patients with fatigue showed involvement of sensorimotor- and frontal network ( $\mathrm{Li}$ et al., 2017; Tessitore et al., 2016; Zhang et al., 2017) indicating that behavioral variability might reflect periods of fatique or maybe fluctuations in fatique. Moreover, decreased connectivity in prefrontal cortex and parieto-occipital junction in PD patients with $\mathrm{MCl}$ and inferior frontal cortex in demented PD patients indicates that fronto-parietal involvement reflects executive dysfunction in behavioral variability. Furthermore, PD patients with impulse control disorders are also characterized by involvement of somatosensory and prefrontal cortices in response to inhibitory demanding stimuli (Politis et al., 2013) and structural changes in meso-limibic, motor and frontal circuits indicating a disconnection between associative, sensorimotor and cognitive networks (Imperiale et al., 2018). Hypoconnectivity between somatomotor- and fronto-parietal network reflects executive dysfunction, and probably more specific changes in fatique and inhibitory control which are reflected by behavioral varibility. Thus, hypoconnectivity between somatomotor- and fronto-parietal networks in combination with task derived behavioral variability might be a promising marker of changes in executive function in PD.

\subsection{Top-down processing in visual misperceptions and behavioral variability in}

\section{Parkinson's Disease}

Attentional and executive dysfunction seem to contribute to development of visual misperceptions (Shine, Keogh, et al., 2015) and behavioral variability in PD. Reduced functional connectivity between attentional networks in visual misperceptions and reduced connectivity to fronto-parietal network in behavioral variabiltiy indicate 
impairments in top-down executive control. Given that minor halluciations precede VH (Fénelon et al., 2000) and that hallucinating PD patients show increase in perceptual errors (Miloserdov, et al., submitted; Shine, Keogh, et al., 2015) perceptual errors might not be corrected by redirecting attention due to disconnection with the DAN (Shine, Keogh, et al., 2015) and relying on the saliency of stimuli (Seeley et al., 2007) leading to manifestation of the perceptual error as misperception of reality. Impairments of topdown modulation from the fronto-parietal network resulting in increased behavioral variabiltiy might be explained by disinhition and fluctuations in arousal. Thus, both, visual misperceptions and behavioral variability might result from deficits in dop-down processes. Tools measuring different levels of these top-down dysfunction in visual misperceptions and behavioral variability might contribute to differential diagnosis in PD.

\subsection{Diagnostic Tools for differential diagnosis of different PD phenotypes}

Cognitive changes seems to be a common component to visual mispercpetions and behaviroal variability and can be assessed by standardized rating scales such as MMSE, FAB, Mattis Dementia Rating Scale, MDR-UPDRS (Kulisevsky \& Pagonabarraga, 2009). Regarding hallucinations specific scales assesing different domains such as visual, auditory, tactile and olfactory are still needed. Especially for minor hallucinations such as misperceptions and passage hallucinations standardized rating scales are missing. Our task derived measures provide a possible tool for objective assessment of visual misperceptions as measured by perceptual errors and cognitive changes as measured by behavioral variability. Possible new candidates for objective and unbiased assessment are EEG task correlates such as P1, P3 amplitude, theta power change and neural variability which need further investigation in PD and 
other groups with executive dysfunction such as ADHD, PD-MCI, ICD and OCD. Resting state measurements might be quite promising objective and convenient diagnostic tools without possible confounds of misunderstanding task instructions or the tendency to perform in a desirable way. Possible promising markers of cognitive decline and VH might be derived from Resting state functional connectivity with hypoconnectivity between somatomotor- and fronto-parietal network and disconnection between attentional networks. Successful recognition of patient groups at risk for $\mathrm{VH}$ and cognitive decline might influence treatment choices and could be beneficial in management of non-motor symptoms during disease progression.

\subsection{Implications for Treatment}

Pharmacological treatment for the prominent motor symptoms consist among other options of dopaminergic mediaction with levodopa and dopamine agonists. Levodopa therapy can improve cognitive flexibility but worsen executive functions (Dirnberger \& Jahanshahi, 2013). PD patients treated with dopamine agonist reported higher frequency of hallucinations (Baker et al., 2009; Stowe et al., 2008). Patients suffering from cognitive decline and $\mathrm{VH}$ show improvement under cholinesterase inhibitors (Emre et al., 2004; Litvinenko, Odinak, Mogilnaya, \& Emelin, 2008). Little evidence is available on antidepressants with citalopram and risperidone showing improvements (Pollock et al., 2007). VH in PD improve under certain antipsychotics such as Clozapine (Parkinson Study Group, 1999; Pollak et al., 2004) and risperidone, which has adverse impact on motor symptoms (Ellis, Cudkowicz, Sexton, \& Growdon, 2000; Ford, Lynch, \& Greene, 1994; Iketani, Kawasaki, \& Yamada, 2017). Brain stimulation, such as electroconvulsive therapy is seldom applied and shows inconsistent results on improvement of psychotic 
symptoms (Burton et al., 2006; Nishioka et al., 2014). Non-invasive brain stimulation shows conflicting results on improvement of cognitive state in PD (Hindle, Petrelli, Clare, \& Kalbe, 2013). These treatment studies indicate that for PD patients at risk for cognitive decline levodopa dose and for PD patients at risk for visual misperceptions and VH dopamine agonist dose should be chosen carefully. Both non-symptoms profiles seem to benefit from cholinesterase inhibitors such as rivastigmine. Pharmacological treatment is of course by now the main strategy. However, non-pharmacological treatment might be an effective complementary therapy and might gain on importance in the case that PD pateint groups at risk for cognitive decline and $\mathrm{VH}$ would be recognized at an early stage when symptoms are quite subtle. Cognitive training improves different cognitive domains such as working memory, executive functioning and processing speed (Leung et al., 2015). Non-pharmacological treatment can also have positive effects on other non-motor symptoms such as $\mathrm{VH}$ by treatement aimed to reduced primary visual deficits (Singh \& Sørensen, 2011). Coping strategies to reduce hallucinatory experience include focusing on the VH or focusing on something different (Diederich, Pieri, \& Goetz, 2003). Moreover, an unexpected finding of a medication study was that social interactions in the setting of a brief psychological treatment improved psychotic symptoms (Swann et al., 2011). Non-pharmacological treatment for cognitive decline and VH might be beneficial at an early disease stage. Unfortunately the potential of cognitive, behavioral and psychological interventions is understudied.

\subsection{Limitations}

One limitation considers the generazability of our findings. Especially the functional connectivity findings of our clinical study described in Chapter 2 is limited by the small 
sample size of sixteen PD patients. For this reason we could not correct for multiple tests and because of the low statistical power the generalizability of the negative corelational results is also limited. Another limitation of generalizability is healthy aging as a model for investigation of possible new marker of cognitive decline such as behavioral, neural and cardiac variability. Expecially with respect to neural variability where insights from previous research in healthy young adults is very limited approaching its potential as an objective measure of cognitive decline in healthy elderly was very useful to explore task related parameters. However, we cannot ignore the possiblity that development of cognitive decline in PD might differ from healthy aging.

\subsubsection{Behavioral Variability}

Another limitations is the debateable question of the source of behavioral variability. Evidence from $A D H D$ research shows that behavioral variability might be related to deficits in attention (Wåhlstedt, Thorell, \& Bohlin, 2009) and or inhibitory control (Buzy, Medoff, \& Schweitzer, 2009; Epstein et al., 2003) or their interaction (Clarke et al., 2007). In ADHD behavioral variability might reflect changes in impulsivity rather than attentional fluctuations (Kofler et al., 2013). However, more research is needed to clarify the cognitive domain reflected by behavioral variability. Furthermore, we did not investigate whether certain stimuli might be more sensitive in provoking behavioral variability. Having a closer look at difference in behavioral variability for different stimuli, PD patients with VH show higher behavioral variability for faces and healthy elderly show a correlation between higher variability for faces and worse performance on the Trail Making Test, indicating that facial stimuli might provoke more variable responses in individuals with more cognitive decline. Given that PD patients show more deficits in recognition of negative emotions (Baggio et al., 2015; Sprengelmeyer et al., 2003) 
investigating influence of facial emotional stimuli on behavioral variability might be promising for detecting different stages of cognitive decline in PD patients.

\subsubsection{Visual hallucinations}

Another limitation is the phenomenology of hallucinatory experience in PD patients. VH do not always occur solitary but for a subgroup of patients can be a multimodal experience combined with auditory and tactile unreal experiences (Kulick, Montgomery, \& Nirenberg, 2018). A multimodal task would provide more sensibility and generazibility to the subgroup of PD patients at risk for multimodal hallucinations. Moreover, given that feeling of presence and passage hallucinations (brief vision of someone or something passing sideways) are more frequent than visual misperceptions (Pagonabarraga et al., 2016) a task which measures perceptual errors of the passage phenomenon probably using peripheral movement might be more sensitive and provide an early, maybe even premotor, objective behavioral marker of VH in PD.

\subsubsection{Resting state}

The final limitation considers the Resting state measuresment. Resting state with eye closed might be problematic as volunteers could move the head slightly (Power, Barnes, Snyder, Schlaggar, \& Petersen, 2012), fall asleep (Tagliazucchi \& Laufs, 2014) and find it difficult to be left without a task just staying still (Vanderwal et al., 2015). PD patients might move the head more, fall asleep more often and be more distressed without an explicit task compared to Healthy Elderly. This might result in differences which influence the measure of Resting state functional connectivity. An alternative approach which adresses the above described sources of unwanted variation might be a paradigm using a neutral movie (Vanderwal et al., 2015). 


\subsection{Conclusion}

We found promising behavioral and neural candidates for objective and quantifiable measurements ofg cognitive decline in Healthy Elderly and $\mathrm{VH}$ and cognitive changes in PD patients. VH could be objectively indicated by perceptual error scores derived from psychophysical perfromance and functional resting state connectivity between the dorsal attention network and the salience network (Shine, Keogh, et al., 2015). Generalizing from our findings in age related cognitive changes in Healthy Elderly and task performance in PD patients, these cognitive changes might be objectively indicated by behavioral variability and neural variability in response to psychophysical stimuli and functional resting state connectivity between the somatomotor- and the fronto-parietal network. Using psychophysical tasks, in combination with EEG and resting state fMRI different PD phenotypes might be detected at an earlier stage of the disease and probably benefit during the clincial course of the disease by phenotype specified treatment. Development of diagnostic tools to facilitate objective identification of different sympotmatic PD phenotypes at an early or even subclinical stage would provide the possibility to identify patients at risk for $\mathrm{VH}$ and cognitive decline. These patients could be monitored more cafully to apply more appropriate treatment and support for patients and their relatives to sustain quality of life as long as possible and to reduce the burden of different non-motor PD symptoms. Future research needs to face the challenge of proving measurement reliability in neuropsychological assessment of non-motor symptoms and sensitivity of perceptual error score, intra-individual behavioral variability, cardiac variability, neural variability and resting state functional connectivity in PD to improve differential diagnosis in non-motor symptoms such as visual misperceptions and 
cognitive changes of PD hopefully one day at an earlier stage probably even premotor stage. 


\section{Bibliography}

Aarsland, D., Larsen, J. P., Lim, N. G., Janvin, C., Karlsen, K., Tandberg, E., \& Cummings, J. L. (1999). Range of neuropsychiatric disturbances in patients with Parkinson's disease. Journal of Neurology, Neurosurgery, and Psychiatry, 67(4), 492-496.

Abbott, R. D., Petrovitch, H., White, L. R., Masaki, K. H., Tanner, C. M., Curb, J. D., ... Ross, G. W. (2001). Frequency of bowel movements and the future risk of Parkinson's disease. Neurology, 57(3), 456-462.

Abhishekh, H. A., Nisarga, P., Kisan, R., Meghana, A., Chandran, S., Trichur Raju, null, \& Sathyaprabha, T. N. (2013). Influence of age and gender on autonomic regulation of heart. Journal of Clinical Monitoring and Computing, 27(3), 259-264. https://doi.org/10.1007/s10877-012-9424-3

Abramovitch, A., McCormack, B., Brunner, D., Johnson, M., \& Wofford, N. (2019). The impact of symptom severity on cognitive function in obsessive-compulsive disorder: A meta-analysis. Clinical Psychology Review, 67, 36-44. https://doi.org/10.1016/j.cpr.2018.09.003

Adler, C. H. (2011). Premotor symptoms and early diagnosis of Parkinson's disease. The International Journal of Neuroscience, 121 Suppl 2, 3-8. https://doi.org/10.3109/00207454.2011.620192

Alonso, A., Huang, X., Mosley, T. H., Heiss, G., \& Chen, H. (2015). Heart rate variability and the risk of Parkinson disease: The Atherosclerosis Risk in Communities study. Annals of Neurology, 77(5), 877-883. https://doi.org/10.1002/ana.24393

Arazi, A., Censor, N., \& Dinstein, I. (2017). Neural Variability Quenching Predicts Individual Perceptual Abilities. The Journal of Neuroscience: The Official Journal 
of the Society for Neuroscience, 37(1), 97-109.

https://doi.org/10.1523/JNEUROSCI.1671-16.2016

Arazi, A., Gonen-Yaacovi, G., \& Dinstein, I. (2017). The Magnitude of Trial-By-Trial Neural Variability Is Reproducible over Time and across Tasks in Humans. ENeuro, 4(6). https://doi.org/10.1523/ENEURO.0292-17.2017

Arias, P., Robles-García, V., Espinosa, N., Corral, Y., \& Cudeiro, J. (2012). Validity of the finger tapping test in Parkinson's disease, elderly and young healthy subjects: is there a role for central fatigue? Clinical Neurophysiology: Official Journal of the International Federation of Clinical Neurophysiology, 123(10), 2034-2041. https://doi.org/10.1016/j.clinph.2012.04.001

Armstrong, R. A. (2011). Visual Symptoms in Parkinson's Disease. Parkinson's Disease, 2011. https://doi.org/10.4061/2011/908306

Aronson, D., \& Burger, A. J. (2001). Effect of beta-blockade on heart rate variability in decompensated heart failure. International Journal of Cardiology, 79(1), 31-39.

Baggio, H. C., Segura, B., Sala-Llonch, R., Marti, M. J., Valldeoriola, F., Compta, Y., ... Junqué, C. (2015). Cognitive impairment and resting-state network connectivity in Parkinson's disease. Human Brain Mapping, 36(1), 199-212.

https://doi.org/10.1002/hbm.22622

Baker, W. L., Silver, D., White, C. M., Kluger, J., Aberle, J., Patel, A. A., \& Coleman, C. I. (2009). Dopamine agonists in the treatment of early Parkinson's disease: a metaanalysis. Parkinsonism \& Related Disorders, 15(4), 287-294. https://doi.org/10.1016/j.parkreldis.2008.07.004

Bartlett, M. S. (1947). The Use of Transformations. Biometrics, 3(1), 39-52. https://doi.org/10.2307/3001536 
Beck, A. T., Ward, C. H., Mendelson, M., Mock, J., \& Erbaugh, J. (1961). An inventory for measuring depression. Archives of General Psychiatry, 4, 561-571.

Becker, C., Gramann, K., Müller, H. J., \& Elliott, M. A. (2009). Electrophysiological correlates of flicker-induced color hallucinations. Consciousness and Cognition, 18(1), 266-276. https://doi.org/10.1016/j.concog.2008.05.001

Beckmann, C. F., Mackay, C. E., Filippini, N., \& Smith, S. M. (2009). Group comparison of resting-state FMRI data using multi-subject ICA and dual regression. Neuroimage, 47(Suppl 1), S148.

Behzadi, Y., Restom, K., Liau, J., \& Liu, T. T. (2007). A component based noise correction method (CompCor) for BOLD and perfusion based fMRI. Neurolmage, 37(1), 90-101. https://doi.org/10.1016/j.neuroimage.2007.04.042

Bellgrove, M. A., Hester, R., \& Garavan, H. (2004). The functional neuroanatomical correlates of response variability: evidence from a response inhibition task. Neuropsychologia, 42(14), 1910-1916. https://doi.org/10.1016/j.neuropsychologia.2004.05.007

Bertram, K., \& Williams, D. R. (2012). Visual hallucinations in the differential diagnosis of parkinsonism. Journal of Neurology, Neurosurgery, and Psychiatry, 83(4), 448452. https://doi.org/10.1136/jnnp-2011-300980

Bielak, A. A., Hultsch, D. F., Strauss, E., MacDonald, S. W., \& Hunter, M. A. (2010). Intraindividual variability in reaction time predicts cognitive outcomes 5 years later. Neuropsychology, 24(6), 731.

Biswal, B., Yetkin, F. Z., Haughton, V. M., \& Hyde, J. S. (1995). Functional connectivity in the motor cortex of resting human brain using echo-planar MRI. Magnetic Resonance in Medicine, 34(4), 537-541. 
Bonanni, L., Thomas, A., Tiraboschi, P., Perfetti, B., Varanese, S., \& Onofrj, M. (2008). EEG comparisons in early Alzheimer's disease, dementia with Lewy bodies and Parkinson's disease with dementia patients with a 2-year follow-up. Brain, 131(3), 690-705. https://doi.org/10.1093/brain/awm322

Bosboom, J. L. W., Stoffers, D., Stam, C. J., Berendse, H. W., \& Wolters, E. C. (2009). Cholinergic modulation of MEG resting-state oscillatory activity in Parkinson's disease related dementia. Clinical Neurophysiology: Official Journal of the International Federation of Clinical Neurophysiology, 120(5), 910-915. https://doi.org/10.1016/j.clinph.2009.03.004

Bourisly, A. K. (2016). Effects of aging on P300 between late young-age and early middle-age adulthood: an electroencephalogram event-related potential study. Neuroreport, 27(14), 999-1003. https://doi.org/10.1097/WNR.0000000000000644

Braak, H., \& Del Tredici, K. (2017). Neuropathological Staging of Brain Pathology in Sporadic Parkinson's disease: Separating the Wheat from the Chaff. Journal of Parkinson's Disease, 7(s1), S71-S85. https://doi.org/10.3233/JPD-179001

Britton, A., Singh-Manoux, A., Hnatkova, K., Malik, M., Marmot, M. G., \& Shipley, M. (2008). The association between heart rate variability and cognitive impairment in middle-aged men and women. The Whitehall II cohort study. Neuroepidemiology, 31(2), 115-121. https://doi.org/10.1159/000148257

Broen, M. P. G., Narayen, N. E., Kuijf, M. L., Dissanayaka, N. N. W., \& Leentjens, A. F. G. (2016). Prevalence of anxiety in Parkinson's disease: A systematic review and meta-analysis. Movement Disorders: Official Journal of the Movement Disorder Society, 31(8), 1125-1133. https://doi.org/10.1002/mds.26643 
Burton, C. L., Strauss, E., Hultsch, D. F., Moll, A., \& Hunter, M. A. (2006). Intraindividual variability as a marker of neurological dysfunction: a comparison of Alzheimer's disease and Parkinson's disease. Journal of Clinical and Experimental Neuropsychology, 28(1), 67-83. https://doi.org/10.1080/13803390490918318

Buzy, W. M., Medoff, D. R., \& Schweitzer, J. B. (2009). Intra-individual variability among children with ADHD on a working memory task: an ex-Gaussian approach. Child Neuropsychology: A Journal on Normal and Abnormal Development in Childhood and Adolescence, 15(5), 441-459. https://doi.org/10.1080/09297040802646991 Cabeza, R., \& Nyberg, L. (2000). Imaging cognition II: An empirical review of 275 PET and fMRI studies. Journal of Cognitive Neuroscience, 12(1), 1-47.

Camicioli, R. M., Wieler, M., de Frias, C. M., \& Martin, W. R. W. (2008). Early, untreated Parkinson's disease patients show reaction time variability. Neuroscience Letters, 441(1), 77-80. https://doi.org/10.1016/j.neulet.2008.06.004

Campbell, M. C., Koller, J. M., Snyder, A. Z., Buddhala, C., Kotzbauer, P. T., \& Perlmutter, J. S. (2015). CSF proteins and resting-state functional connectivity in Parkinson disease. Neurology, 84(24), 2413-2421. https://doi.org/10.1212/WNL.0000000000001681

Carmel, D., Arcaro, M., Kastner, S., \& Hasson, U. (2010). How to create and use binocular rivalry. Journal of Visualized Experiments: JoVE, (45). https://doi.org/10.3791/2030

Caviness, J. N., Hentz, J. G., Evidente, V. G., Driver-Dunckley, E., Samanta, J., Mahant, P., ... Adler, C. H. (2007). Both early and late cognitive dysfunction affects the electroencephalogram in Parkinson's disease. Parkinsonism \& Related Disorders, 13(6), 348-354. https://doi.org/10.1016/j.parkreldis.2007.01.003 
Caviness, J. N., Utianski, R. L., Hentz, J. G., Beach, T. G., Dugger, B. N., Shill, H. A., ... Adler, C. H. (2016). Differential spectral quantitative electroencephalography patterns between control and Parkinson's disease cohorts. European Journal of Neurology, 23(2), 387-392. https://doi.org/10.1111/ene.12878

Chapleau, M. W., \& Sabharwal, R. (2011). Methods of assessing vagus nerve activity and reflexes. Heart Failure Reviews, 16(2), 109-127. https://doi.org/10.1007/s10741-010-9174-6

Chaudhuri, K. R., Martinez-Martin, P., Brown, R. G., Sethi, K., Stocchi, F., Odin, P., ... Schapira, A. H. V. (2007). The metric properties of a novel non-motor symptoms scale for Parkinson's disease: Results from an international pilot study. Movement Disorders: Official Journal of the Movement Disorder Society, 22(13), 1901-1911. https://doi.org/10.1002/mds.21596

Chaudhuri, K. R., Martinez-Martin, P., Schapira, A. H. V., Stocchi, F., Sethi, K., Odin, P., ... Olanow, C. W. (2006). International multicenter pilot study of the first comprehensive self-completed nonmotor symptoms questionnaire for Parkinson's disease: the NMSQuest study. Movement Disorders: Official Journal of the Movement Disorder Society, 21(7), 916-923. https://doi.org/10.1002/mds.20844 Cherbuin, N., Sachdev, P., \& Anstey, K. J. (2010). Neuropsychological predictors of transition from healthy cognitive aging to mild cognitive impairment: The PATH through life study. The American Journal of Geriatric Psychiatry: Official Journal of the American Association for Geriatric Psychiatry, 18(8), 723-733.

Churchland, M. M., Byron, M. Y., Cunningham, J. P., Sugrue, L. P., Cohen, M. R......Shenoy, K. V. (2010). Stimulus onset quenches neural variability: a 
widespread cortical phenomenon. Nat Neurosci. 13(3), 369-378.

https://doi:10.1038/nn.2501

Clarke, S. D., Kohn, M. R., Hermens, D. F., Rabbinge, M., Clark, C. R., Gordon, E., \& Williams, L. M. (2007). Distinguishing symptom profiles in adolescent ADHD using an objective cognitive test battery. International Journal of Adolescent Medicine and Health, 19(3), 355-367.

Collerton, D., Perry, E., \& McKeith, I. (2005). Why people see things that are not there: a novel Perception and Attention Deficit model for recurrent complex visual hallucinations. The Behavioral and Brain Sciences, 28(6), 737-757; discussion 757-794. https://doi.org/10.1017/S0140525X05000130

Cosgrove, J., Alty, J. E., \& Jamieson, S. (2015). Cognitive impairment in Parkinson's disease. Postgraduate Medical Journal, 91(1074), 212-220. https://doi.org/10.1136/postgradmedj-2015-133247

Costa, A. S., Dogan, I., Schulz, J. B., \& Reetz, K. (2019). Going beyond the mean: Intraindividual variability of cognitive performance in prodromal and early neurodegenerative disorders. The Clinical Neuropsychologist, 1-21. https://doi.org/10.1080/13854046.2018.1533587

Cozac, V. V., Chaturvedi, M., Hatz, F., Meyer, A., Fuhr, P., \& Gschwandtner, U. (2016). Increase of EEG Spectral Theta Power Indicates Higher Risk of the Development of Severe Cognitive Decline in Parkinson's Disease after 3 Years. Frontiers in Aging Neuroscience, 8, 284. https://doi.org/10.3389/fnagi.2016.00284

Creese, B., Albertyn, C. P., Dworkin, S., Thomas, R. S., Wan, Y. M., \& Ballard, C. (2018). Executive function but not episodic memory decline associated with visual 
hallucinations in Parkinson's disease. Journal of Neuropsychology. https://doi.org/10.1111/jnp.12169

Cummins, T. D. R., Broughton, M., \& Finnigan, S. (2008). Theta oscillations are affected by amnestic mild cognitive impairment and cognitive load. International Journal of Psychophysiology: Official Journal of the International Organization of Psychophysiology, 70(1), 75-81. https://doi.org/10.1016/j.jpsycho.2008.06.002 daSilva Morgan, K., Elder, G. J., Ffytche, D. H., Collerton, D., \& Taylor, J.-P. (2018). The utility and application of electrophysiological methods in the study of visual hallucinations. Clinical Neurophysiology: Official Journal of the International Federation of Clinical Neurophysiology, 129(11), 2361-2371. https://doi.org/10.1016/j.clinph.2018.08.019

de Frias, C. M., Dixon, R. A., \& Camicioli, R. (2012). Neurocognitive speed and inconsistency in Parkinson's disease with and without incipient dementia: an 18month prospective cohort study. Journal of the International Neuropsychological Society: JINS, 18(4), 764-772. https://doi.org/10.1017/S1355617712000422 de Frias, C. M., Dixon, R. A., Fisher, N., \& Camicioli, R. (2007). Intraindividual variability in neurocognitive speed: a comparison of Parkinson's disease and normal older adults. Neuropsychologia, 45(11), 2499-2507. https://doi.org/10.1016/j.neuropsychologia.2007.03.022 de la Riva, P., Smith, K., Xie, S. X., \& Weintraub, D. (2014). Course of psychiatric symptoms and global cognition in early Parkinson disease. Neurology, 83(12), 1096-1103. https://doi.org/10.1212/WNL.0000000000000801

Deary, I. J., \& Der, G. (2005). Reaction Time, Age, and Cognitive Ability: Longitudinal Findings from Age 16 to 63 Years in Representative Population Samples. Aging, 
Neuropsychology, and Cognition, 12(2), 187-215.

https://doi.org/10.1080/13825580590969235

Diederich, N. J., Fénelon, G., Stebbins, G., \& Goetz, C. G. (2009). Hallucinations in

Parkinson disease. Nature Reviews. Neurology, 5(6), 331-342.

https://doi.org/10.1038/nrneurol.2009.62

Diederich, N. J., Pieri, V., \& Goetz, C. G. (2003). Coping strategies for visual

hallucinations in Parkinson's disease. Movement Disorders: Official Journal of the Movement Disorder Society, 18(7), 831-832. https://doi.org/10.1002/mds.10450

Diederich, N. J., Raman, R., Leurgans, S., \& Goetz, C. G. (2002). Progressive worsening of spatial and chromatic processing deficits in Parkinson disease. Archives of Neurology, 59(8), 1249-1252.

Dinstein, I., Heeger, D. J., \& Behrmann, M. (2015). Neural variability: friend or foe? Trends in Cognitive Sciences, 19(6), 322-328. https://doi.org/10.1016/j.tics.2015.04.005

Dirnberger, G., \& Jahanshahi, M. (2013). Executive dysfunction in Parkinson's disease: a review. Journal of Neuropsychology, 7(2), 193-224. https://doi.org/10.1111/jnp.12028

Dixon, R. A., Garrett, D. D., Lentz, T. L., MacDonald, S. W. S., Strauss, E., \& Hultsch, D. F. (2007). Neurocognitive markers of cognitive impairment: exploring the roles of speed and inconsistency. Neuropsychology, 21(3), 381-399. https://doi.org/10.1037/0894-4105.21.3.381

Dömötör, Z., Szemerszky, R., \& Köteles, F. (2015). Subjective and objective effects of coffee consumption - caffeine or expectations? Acta Physiologica Hungarica, 102(1), 77-85. https://doi.org/10.1556/APhysiol.101.2014.012 
Dudley, R., Wood, M., Spencer, H., Brabban, A., Mosimann, U. P., \& Collerton, D. (2012). Identifying specific interpretations and use of safety behaviours in people with distressing visual hallucinations: an exploratory study. Behavioural and Cognitive Psychotherapy, 40(3), 367-375.

https://doi.org/10.1017/S1352465811000750

Dykiert, D., Der, G., Starr, J. M., \& Deary, I. J. (2012). Age differences in intra-individual variability in simple and choice reaction time: systematic review and metaanalysis. PloS One, 7(10), e45759. https://doi.org/10.1371/journal.pone.0045759

Ekman, P., \& Friesen, W. V. (1976). Pictures of Facial Affect. Palo Alto.CA.Colsulting Psychologists Press.

Ellis, T., Cudkowicz, M. E., Sexton, P. M., \& Growdon, J. H. (2000). Clozapine and risperidone treatment of psychosis in Parkinson's disease. The Journal of Neuropsychiatry and Clinical Neurosciences, 12(3), 364-369. https://doi.org/10.1176/jnp.12.3.364

Emre, M., Aarsland, D., Albanese, A., Byrne, E. J., Deuschl, G., De Deyn, P. P., ... Lane, R. (2004). Rivastigmine for dementia associated with Parkinson's disease. The New England Journal of Medicine, 351(24), 2509-2518. https://doi.org/10.1056/NEJMoa041470

Epstein, J. N., Erkanli, A., Conners, C. K., Klaric, J., Costello, J. E., \& Angold, A. (2003). Relations between Continuous Performance Test performance measures and ADHD behaviors. Journal of Abnormal Child Psychology, 31(5), 543-554.

Eversfield, C. L., \& Orton, L. D. (2018). Auditory and visual hallucination prevalence in Parkinson's disease and dementia with Lewy bodies: a systematic review and 
meta-analysis. Psychological Medicine, 1-12.

https://doi.org/10.1017/S0033291718003161

Falkenstein, M., Yordanova, J., \& Kolev, V. (2006). Effects of aging on slowing of motorresponse generation. International Journal of Psychophysiology: Official Journal of the International Organization of Psychophysiology, 59(1), 22-29. https://doi.org/10.1016/j.jpsycho.2005.08.004

Fassbender, C., Lesh, T. A., Ursu, S., \& Salo, R. (2015). Reaction Time Variability and Related Brain Activity in Methamphetamine Psychosis. Biological Psychiatry, 77(5), 465-474. https://doi.org/10.1016/j.biopsych.2014.07.028

Fassbender, C., Scangos, K., Lesh, T. A., \& Carter, C. S. (2014). RT distributional analysis of cognitive-control-related brain activity in first-episode schizophrenia. Cognitive, Affective \& Behavioral Neuroscience, 14(1), 175-188. https://doi.org/10.3758/s13415-014-0252-4

Fénelon, G., Mahieux, F., Huon, R., \& Ziégler, M. (2000). Hallucinations in Parkinson's disease: prevalence, phenomenology and risk factors. Brain: A Journal of Neurology, 123 ( Pt 4), 733-745.

Fereshtehnejad, S.-M., Romenets, S. R., Anang, J. B. M., Latreille, V., Gagnon, J.-F., \& Postuma, R. B. (2015). New Clinical Subtypes of Parkinson Disease and Their Longitudinal Progression: A Prospective Cohort Comparison With Other Phenotypes. JAMA Neurology, 72(8), 863-873. https://doi.org/10.1001/jamaneurol.2015.0703

Ferini-Strambi, L., Franceschi, M., Pinto, P., Zucconi, M., \& Smirne, S. (1992).

Respiration and heart rate variability during sleep in untreated Parkinson patients. Gerontology, 38(1-2), 92-98. https://doi.org/10.1159/000213312 
Fernandez, H. H., Aarsland, D., Fénelon, G., Friedman, J. H., Marsh, L., Tröster, A. I., ... Goetz, C. G. (2008). Scales to assess psychosis in Parkinson's disease: Critique and recommendations. Movement Disorders: Official Journal of the Movement Disorder Society, 23(4), 484-500. https://doi.org/10.1002/mds.21875

Feuerriegel, D., Churches, O., Hofmann, J., \& Keage, H. A. D. (2015). The N170 and face perception in psychiatric and neurological disorders: A systematic review. Clinical Neurophysiology: Official Journal of the International Federation of Clinical Neurophysiology, 126(6), 1141-1158.

https://doi.org/10.1016/j.clinph.2014.09.015

Filipović, S. R., Covicković-Sternić, N., Stojanović-Svetel, M., Lecić, D., \& Kostić, V. S. (1998). Depression in Parkinson's disease: an EEG frequency analysis study. Parkinsonism \& Related Disorders, 4(4), 171-178.

Finnigan, S., \& Robertson, I. H. (2011). Resting EEG theta power correlates with cognitive performance in healthy older adults. Psychophysiology, 48(8), 10831087. https://doi.org/10.1111/j.1469-8986.2010.01173.x

Flehmig, H. C., Steinborn, M., Langner, R., Scholz, A., \& Westhoff, K. (2007). Assessing intraindividual variability in sustained attention: reliability, relation to speed and accuracy, and practice effects. Psychology Science, 49(2), 132-149.

Folstein, M. F., Folstein, S. E., \& McHugh, P. R. (1975). "Mini-mental state". A practical method for grading the cognitive state of patients for the clinician. Journal of Psychiatric Research, 12(3), 189-198.

Foltynie, T., Brayne, C. E. G., Robbins, T. W., \& Barker, R. A. (2004). The cognitive ability of an incident cohort of Parkinson's patients in the UK. The CamPaIGN 
study. Brain: A Journal of Neurology, 127(Pt 3), 550-560.

https://doi.org/10.1093/brain/awh067

Fonseca, L. C., Tedrus, G. M. a. S., Letro, G. H., \& Bossoni, A. S. (2009). Dementia, mild cognitive impairment and quantitative EEG in patients with Parkinson's disease. Clinical EEG and Neuroscience, 40(3), 168-172.

https://doi.org/10.1177/155005940904000309

Ford, B., Lynch, T., \& Greene, P. (1994). Risperidone in Parkinson's disease. Lancet (London, England), 344(8923), 681.

Franciotti, R., Delli Pizzi, S., Perfetti, B., Tartaro, A., Bonanni, L., Thomas, A., ... Onofrj, M. (2015). Default mode network links to visual hallucinations: A comparison between Parkinson's disease and multiple system atrophy. Movement Disorders: Official Journal of the Movement Disorder Society, 30(9), 1237-1247. https://doi.org/10.1002/mds.26285

Franciotti, R., Falasca, N. W., Bonanni, L., Anzellotti, F., Maruotti, V., Comani, S., ... Onofrj, M. (2013). Default network is not hypoactive in dementia with fluctuating cognition: an Alzheimer disease/dementia with Lewy bodies comparison. Neurobiology of Aging, 34(4), 1148-1158. https://doi.org/10.1016/j.neurobiolaging.2012.09.015

Frewen, J., Finucane, C., Savva, G. M., Boyle, G., Coen, R. F., \& Kenny, R. A. (2013). Cognitive function is associated with impaired heart rate variability in ageing adults: the Irish longitudinal study on ageing wave one results. Clinical Autonomic Research: Official Journal of the Clinical Autonomic Research Society, 23(6), 313-323. https://doi.org/10.1007/s10286-013-0214-x 
Gagnon, J.-F., Fantini, M. L., Bédard, M.-A., Petit, D., Carrier, J., Rompré, S., ... Montplaisir, J. (2004). Association between waking EEG slowing and REM sleep behavior disorder in PD without dementia. Neurology, 62(3), 401-406.

Garrett, D. D., Kovacevic, N., McIntosh, A. R., \& Grady, C. L. (2013). The modulation of BOLD variability between cognitive states varies by age and processing speed. Cerebral Cortex (New York, N.Y.: 1991), 23(3), 684-693. https://doi.org/10.1093/cercor/bhs055

Geerligs, L., Saliasi, E., Maurits, N. M., Renken, R. J., \& Lorist, M. M. (2014). Brain mechanisms underlying the effects of aging on different aspects of selective attention. Neurolmage, 91, 52-62.

https://doi.org/10.1016/j.neuroimage.2014.01.029

Geraedts, V. J., Boon, L. I., Marinus, J., Gouw, A. A., van Hilten, J. J., Stam, C. J., ... Contarino, M. F. (2018). Clinical correlates of quantitative EEG in Parkinson disease: A systematic review. Neurology, 91(19), 871-883. https://doi.org/10.1212/WNL.0000000000006473

Givens, B. (1996). Stimulus-evoked resetting of the dentate theta rhythm: relation to working memory. Neuroreport, 8(1), 159-163.

Goetz, C. G., \& Stebbins, G. T. (1993). Risk factors for nursing home placement in advanced Parkinson's disease. Neurology, 43(11), 2227-2229.

Goetz, C.G., Tilley, B.C., Shaftman, S.R., Stebbins, G.T., Fahn, S., Martinez-Martin, P., Poewe, W., Sampaio, C., Stern, M.B., Dodel, R., Dubois, B., Holloway, R., Jankovic, J., Kulisevsky, J., Lang, A.E., Lees, A., Leurgans, S., LeWitt, P.A., Nyenhuis, D., Olanow, C.W., Rascol, O., Schrag, A., Teresi, J.A., Hilten, J.J. van, LaPelle, N., 2008. Movement Disorder Society-sponsored revision of the Unified 
Parkinson's Disease Rating Scale (MDS-UPDRS): Scale presentation and clinimetric testing results. Movement Disorders 23, 2129-2170. https://doi.org/10.1002/mds.22340

Goldman, J. G., \& Postuma, R. (2014). Premotor and nonmotor features of Parkinson's disease. Current Opinion in Neurology, 27(4), 434-441. https://doi.org/10.1097/WCO.0000000000000112

Goldman, J. G., Stebbins, G. T., Dinh, V., Bernard, B., Merkitch, D., deToledo-Morrell, L., \& Goetz, C. G. (2014). Visuoperceptive region atrophy independent of cognitive status in patients with Parkinson's disease with hallucinations. Brain: A Journal of Neurology, 137(Pt 3), 849-859. https://doi.org/10.1093/brain/awt360

Goodarzi, Z., Mrklas, K. J., Roberts, D. J., Jette, N., Pringsheim, T., \& Holroyd-Leduc, J. (2016). Detecting depression in Parkinson disease: A systematic review and meta-analysis. Neurology, 87(4), 426-437. https://doi.org/10.1212/WNL.0000000000002898 Gorges, M., Müller, H.-P., Lulé, D., LANDSCAPE Consortium, Pinkhardt, E. H., Ludolph, A. C., \& Kassubek, J. (2015). To rise and to fall: functional connectivity in cognitively normal and cognitively impaired patients with Parkinson's disease. Neurobiology of Aging, 36(4), 1727-1735. https://doi.org/10.1016/j.neurobiolaging.2014.12.026 Gorus, E., De Raedt, R., Lambert, M., Lemper, J.-C., \& Mets, T. (2008). Reaction times and performance variability in normal aging, mild cognitive impairment, and Alzheimer's disease. Journal of Geriatric Psychiatry and Neurology, 21(3), 204218. https://doi.org/10.1177/0891988708320973 
Graham, G., Dean, J., Mosimann, U. P., Colbourn, C., Dudley, R., Clarke, M., \& Collerton, D. (2011). Specific attentional impairments and complex visual hallucinations in eye disease. International Journal of Geriatric Psychiatry, 26(3), 263-267. https://doi.org/10.1002/gps.2522

Gratwicke, J., Jahanshahi, M., \& Foltynie, T. (2015). Parkinson's disease dementia: a neural networks perspective. Brain: A Journal of Neurology, 138(Pt 6), 14541476. https://doi.org/10.1093/brain/awv104

Graybiel, A. M. (2005). The basal ganglia: learning new tricks and loving it. Current Opinion in Neurobiology, 15(6), 638-644. https://doi.org/10.1016/j.conb.2005.10.006

Guillaume, C., Guillery-Girard, B., Chaby, L., Lebreton, K., Hugueville, L., Eustache, F., Fiori, N., 2009. The time course of repetition effects for familiar faces and objects: an ERP study. Brain Res. 1248, 149-161. https://doi.org/10.1016/j.brainres.2008.10.069

Gupta, M., Singh, G., Khwaja, G. A., \& Mehndiratta, M. M. (2004). Hallucinations in Parkinson's disease--a study of forty three patients. The Journal of the Association of Physicians of India, 52, 703-706.

Haber, S. N. (2014). The place of dopamine in the cortico-basal ganglia circuit.

Neuroscience, 282, 248-257. https://doi.org/10.1016/j.neuroscience.2014.10.008

Harada, C. N., Natelson Love, M. C., \& Triebel, K. L. (2013). Normal cognitive aging. Clinics in Geriatric Medicine, 29(4), 737-752. https://doi.org/10.1016/j.cger.2013.07.002

Haring, A. E., Zhuravleva, T. Y., Alperin, B. R., Rentz, D. M., Holcomb, P. J., \& Daffner, K. R. (2013). Age-related differences in enhancement and suppression of neural 
activity underlying selective attention in matched young and old adults. Brain Research, 1499, 69-79. https://doi.org/10.1016/j.brainres.2013.01.003

Harper, J., Malone, S. M., \& Iacono, W. G. (2017). Theta- and delta-band EEG network dynamics during a novelty oddball task. Psychophysiology, 54(11), 1590-1605. https://doi.org/10.1111/psyp.12906

Haynes, B. I., Bauermeister, S., \& Bunce, D. (2017). A Systematic Review of Longitudinal Associations Between Reaction Time Intraindividual Variability and Age-Related Cognitive Decline or Impairment, Dementia, and Mortality. Journal of the International Neuropsychological Society: JINS, 23(5), 431-445. https://doi.org/10.1017/S1355617717000236

He, X., Zhang, Y., Chen, J., Xie, C., Gan, R., Wang, L., \& Wang, L. (2017). Changes in theta activities in the left posterior temporal region, left occipital region and right frontal region related to mild cognitive impairment in Parkinson's disease patients. The International Journal of Neuroscience, 127(1), 66-72. https://doi.org/10.3109/00207454.2016.1143823

He, X., Zhang, Y., Chen, J., Xie, C., Gan, R., Yang, R., ... Wang, L. (2017). The patterns of EEG changes in early-onset Parkinson's disease patients. The International Journal of Neuroscience, 127(11), 1028-1035. https://doi.org/10.1080/00207454.2017.1304393

Hedden, T., \& Gabrieli, J. D. E. (2004). Insights into the ageing mind: a view from cognitive neuroscience. Nature Reviews. Neuroscience, 5(2), 87-96. https://doi.org/10.1038/nrn1323

Hely, M. A., Reid, W. G. J., Adena, M. A., Halliday, G. M., \& Morris, J. G. L. (2008). The Sydney multicenter study of Parkinson's disease: the inevitability of dementia at 
20 years. Movement Disorders: Official Journal of the Movement Disorder Society, 23(6), 837-844. https://doi.org/10.1002/mds.21956

Hindle, J. V., Petrelli, A., Clare, L., \& Kalbe, E. (2013). Nonpharmacological enhancement of cognitive function in Parkinson's disease: a systematic review. Movement Disorders: Official Journal of the Movement Disorder Society, 28(8), 1034-1049. https://doi.org/10.1002/mds.25377

Hohenfeld, C., Werner, C. J., \& Reetz, K. (2018). Resting-state connectivity in neurodegenerative disorders: Is there potential for an imaging biomarker? Neurolmage. Clinical, 18, 849-870. https://doi.org/10.1016/j.nicl.2018.03.013 Holroyd, S., Currie, L., \& Wooten, G. F. (2001). Prospective study of hallucinations and delusions in Parkinson's disease. Journal of Neurology, Neurosurgery, and Psychiatry, 70(6), 734-738.

Hsieh, S., \& Lin, Y.-C. (2017). Stopping ability in younger and older adults: Behavioral and event-related potential. Cognitive, Affective \& Behavioral Neuroscience, 17(2), 348-363. https://doi.org/10.3758/s13415-016-0483-7

Huang, C., Mattis, P., Perrine, K., Brown, N., Dhawan, V., \& Eidelberg, D. (2008). Metabolic abnormalities associated with mild cognitive impairment in Parkinson disease. Neurology, 70(16 Pt 2), 1470-1477. https://doi.org/10.1212/01.wnl.0000304050.05332.9c

Hultsch, D. F., MacDonald, S. W. S., \& Dixon, R. A. (2002). Variability in reaction time performance of younger and older adults. The Journals of Gerontology. Series B, Psychological Sciences and Social Sciences, 57(2), P101-115.

Iketani, R., Kawasaki, Y., \& Yamada, H. (2017). Comparative Utility of Atypical Antipsychotics for the Treatment of Psychosis in Parkinson's Disease: A 
Systematic Review and Bayesian Network Meta-analysis. Biological \& Pharmaceutical Bulletin, 40(11), 1976-1982. https://doi.org/10.1248/bpb.b1700602

Imperiale, F., Agosta, F., Canu, E., Markovic, V., Inuggi, A., Jecmenica-Lukic, M., ... Filippi, M. (2018). Brain structural and functional signatures of impulsivecompulsive behaviours in Parkinson's disease. Molecular Psychiatry, 23(2), 459466. https://doi.org/10.1038/mp.2017.18

Iwasa, K., Nakajima, K., Yoshikawa, H., Tada, A., Taki, J., \& Takamori, M. (1998). Decreased myocardial 123I-MIBG uptake in Parkinson's disease. Acta Neurologica Scandinavica, 97(5), 303-306.

Jensen, B. H., Hougaard, A., Amin, F. M., Larsson, H. B. W., \& Ashina, M. (2015). Structural asymmetry of cortical visual areas is related to ocular dominance. Neuroreport, 26(17), 1071-1076. https://doi.org/10.1097/WNR.0000000000000470 Jonas, J., Jacques, C., Liu-Shuang, J., Brissart, H., Colnat-Coulbois, S., Maillard, L., \& Rossion, B. (2016). A face-selective ventral occipito-temporal map of the human brain with intracerebral potentials. Proceedings of the National Academy of Sciences of the United States of America, 113(28), E4088-4097. https://doi.org/10.1073/pnas.1522033113

Kalia, L. V., \& Lang, A. E. (2015). Parkinson's disease. Lancet (London, England), 386(9996), 896-912. https://doi.org/10.1016/S0140-6736(14)61393-3

Kälin, A. M., Pflüger, M., Gietl, A. F., Riese, F., Jäncke, L., Nitsch, R. M., \& Hock, C. (2014). Intraindividual variability across cognitive tasks as a potential marker for 
prodromal Alzheimer's disease. Frontiers in Aging Neuroscience, 6, 147. https://doi.org/10.3389/fnagi.2014.00147

Kallio, M., Suominen, K., Haapaniemi, T., Sotaniemi, K., Myllylä, V. V., Astafiev, S., \& Tolonen, U. (2004). Nocturnal cardiac autonomic regulation in Parkinson's disease. Clinical Autonomic Research: Official Journal of the Clinical Autonomic Research Society, 14(2), 119-124. https://doi.org/10.1007/s10286-004-0173-3

Katzenschlager, R., \& Lees, A. J. (2004). Olfaction and Parkinson's syndromes: its role in differential diagnosis. Current Opinion in Neurology, 17(4), 417-423.

Kelly, A. C., Uddin, L. Q., Biswal, B. B., Castellanos, F. X., \& Milham, M. P. (2008). Competition between functional brain networks mediates behavioral variability. Neuroimage, 39(1), 527-537.

Kertzman, S. G., Poyurovski, M., Faragian, S., Weizman, R., Cohen, K., Aizer, A., ... Dannon, P. N. (2018). Distinct Response Inhibition Patterns in Obsessive Compulsive Disorder Patients and Pathological Gamblers. Frontiers in Psychiatry, 9, 652. https://doi.org/10.3389/fpsyt.2018.00652

Kessler, R. C., Birnbaum, H., Bromet, E., Hwang, I., Sampson, N., \& Shahly, V. (2010). Age differences in major depression: results from the National Comorbidity Survey Replication (NCS-R). Psychological Medicine, 40(2), 225-237. https://doi.org/10.1017/S0033291709990213

Kida, Y., Tachibana, H., Takeda, M., Yoshikawa, H., \& Okita, T. (2007). Recognition memory for unfamiliar faces in Parkinson's disease: behavioral and electrophysiologic measures. Parkinsonism \& Related Disorders, 13(3), 157-164. https://doi.org/10.1016/j.parkreldis.2006.08.012 
Kim, D. H., Lipsitz, L. A., Ferrucci, L., Varadhan, R., Guralnik, J. M., Carlson, M. C., ... Chaves, P. H. M. (2006). Association between reduced heart rate variability and cognitive impairment in older disabled women in the community: Women's Health and Aging Study I. Journal of the American Geriatrics Society, 54(11), 17511757. https://doi.org/10.1111/j.1532-5415.2006.00940.x

Kim, M., Lee, T. H., Kim, J.-H., Hong, H., Lee, T. Y., Lee, Y., ... Kwon, J. S. (2018). Decomposing P300 into correlates of genetic risk and current symptoms in schizophrenia: An inter-trial variability analysis. Schizophrenia Research, 192, 232-239. https://doi.org/10.1016/j.schres.2017.04.001

Kim, Y. E., \& Jeon, B. S. (2014). Clinical implication of REM sleep behavior disorder in Parkinson's disease. Journal of Parkinson's Disease, 4(2), 237-244. https://doi.org/10.3233/JPD-130293

Klassen, B. T., Hentz, J. G., Shill, H. A., Driver-Dunckley, E., Evidente, V. G. H., Sabbagh, M. N., ... Caviness, J. N. (2011). Quantitative EEG as a predictive biomarker for Parkinson disease dementia. Neurology, 77(2), 118-124. https://doi.org/10.1212/WNL.0b013e318224af8d

Koerts, J., Borg, M. A. J. P., Meppelink, A. M., Leenders, K. L., van Beilen, M., \& van Laar, T. (2010). Attentional and perceptual impairments in Parkinson's disease with visual hallucinations. Parkinsonism \& Related Disorders, 16(4), 270-274. https://doi.org/10.1016/j.parkreldis.2010.01.003

Kofler, M. J., Rapport, M. D., Sarver, D. E., Raiker, J. S., Orban, S. A., Friedman, L. M., \& Kolomeyer, E. G. (2013). Reaction time variability in ADHD: a meta-analytic review of 319 studies. Clinical Psychology Review, 33(6), 795-811. https://doi.org/10.1016/j.cpr.2013.06.001 
Kritzinger, C., Vollstedt, E.-J., Hückelheim, K., Lorwin, A., Graf, J., Tunc, S., ... Kasten, M. (2015). Qualitative Characteristics of Depression in Parkinson's Patients and Controls. Behavioural Neurology, 2015, 961372.

https://doi.org/10.1155/2015/961372

Kulick, C. V., Montgomery, K. M., \& Nirenberg, M. J. (2018). Comprehensive identification of delusions and olfactory, tactile, gustatory, and minor hallucinations in Parkinson's disease psychosis. Parkinsonism \& Related Disorders, 54, 40-45. https://doi.org/10.1016/j.parkreldis.2018.04.008

Kulisevsky, J., \& Pagonabarraga, J. (2009). Cognitive impairment in Parkinson's disease: tools for diagnosis and assessment. Movement Disorders: Official Journal of the Movement Disorder Society, 24(8), 1103-1110. https://doi.org/10.1002/mds.22506

Kurita, A., Murakami, M., Takagi, S., Matsushima, M., \& Suzuki, M. (2010). Visual hallucinations and altered visual information processing in Parkinson disease and dementia with Lewy bodies. Movement Disorders: Official Journal of the Movement Disorder Society, 25(2), 167-171. https://doi.org/10.1002/mds.22919

Latreille, V., Carrier, J., Gaudet-Fex, B., Rodrigues-Brazète, J., Panisset, M., Chouinard, S., ... Gagnon, J.-F. (2016). Electroencephalographic prodromal markers of dementia across conscious states in Parkinson's disease. Brain: A Journal of Neurology, 139(Pt 4), 1189-1199. https://doi.org/10.1093/brain/aww018

Lee, W.-J., Tsai, C.-F., Gauthier, S., Wang, S.-J., \& Fuh, J.-L. (2012). The association between cognitive impairment and neuropsychiatric symptoms in patients with Parkinson's disease dementia. International Psychogeriatrics, 24(12), 19801987. https://doi.org/10.1017/S1041610212001317 
Lees, A. J., \& Smith, E. (1983). Cognitive deficits in the early stages of Parkinson's disease. Brain: A Journal of Neurology, 106 (Pt 2), 257-270.

Leung, I. H. K., Walton, C. C., Hallock, H., Lewis, S. J. G., Valenzuela, M., \& Lampit, A. (2015). Cognitive training in Parkinson disease: A systematic review and metaanalysis. Neurology, 85(21), 1843-1851. https://doi.org/10.1212/WNL.0000000000002145

Levin, B. E., Llabre, M. M., Reisman, S., Weiner, W. J., Sanchez-Ramos, J., Singer, C., \& Brown, M. C. (1991). Visuospatial impairment in Parkinson's disease. Neurology, 41(3), 365-369.

Li, J., Yuan, Y., Wang, M., Zhang, J., Zhang, L., Jiang, S., ... Zhang, K. (2017). Alterations in regional homogeneity of resting-state brain activity in fatigue of Parkinson's disease. Journal of Neural Transmission (Vienna, Austria: 1996), 124(10), 1187-1195. https://doi.org/10.1007/s00702-017-1748-1

Li, S. C., Huxhold, O., \& Schmiedek, F. (2004). Aging and attenuated processing robustness. Evidence from cognitive and sensorimotor functioning. Gerontology, 50(1), 28-34. https://doi.org/10.1159/000074386

Linazasoro, G. (2007). Classical Parkinson disease versus Parkinson complex-reflections against staging and in favour of heterogeneity. European Journal of Neurology, 14(7), 721-728. https://doi.org/10.1111/j.1468-1331.2007.01853.x

Lithfous, S., Tromp, D., Dufour, A., Pebayle, T., Goutagny, R., \& Després, O. (2015). Decreased theta power at encoding and cognitive mapping deficits in elderly individuals during a spatial memory task. Neurobiology of Aging, 36(10), 28212829. https://doi.org/10.1016/j.neurobiolaging.2015.07.007 
Litvinenko, I. V., Odinak, M. M., Mogilnaya, V. I., \& Emelin, A. Y. (2008). Efficacy and safety of galantamine (reminyl) for dementia in patients with Parkinson's disease (an open controlled trial). Neuroscience and Behavioral Physiology, 38(9), 937945. https://doi.org/10.1007/s11055-008-9077-3

López, M. E., Cuesta, P., Garcés, P., Castellanos, P. N., Aurtenetxe, S., Bajo, R., ... Fernandez, A. (2014). MEG spectral analysis in subtypes of mild cognitive impairment. Age (Dordrecht, Netherlands), 36(3), 9624. https://doi.org/10.1007/s11357-014-9624-5

Lopez, O. L., Becker, J. T., Brenner, R. P., Rosen, J., Bajulaiye, O. I., \& Reynolds, C. F. (1991). Alzheimer's disease with delusions and hallucinations:

Neuropsychological and electroencephalographic correlates. Neurology, 41(6), 906-912. https://doi.org/10.1212/WNL.41.6.906

Lövdén, M., Li, S.-C., Shing, Y. L., \& Lindenberger, U. (2007). Within-person trial-to-trial variability precedes and predicts cognitive decline in old and very old age: longitudinal data from the Berlin Aging Study. Neuropsychologia, 45(12), 28272838. https://doi.org/10.1016/j.neuropsychologia.2007.05.005

Lubitz, A. F., Niedeggen, M., \& Feser, M. (2017). Aging and working memory performance: Electrophysiological correlates of high and low performing elderly. Neuropsychologia, 106, 42-51. https://doi.org/10.1016/j.neuropsychologia.2017.09.002

Luft, C. D. B., \& Bhattacharya, J. (2015). Aroused with heart: Modulation of heartbeat evoked potential by arousal induction and its oscillatory correlates. Scientific Reports, 5. https://doi.org/10.1038/srep15717 
Luque-Casado, A., Perales, J. C., Cárdenas, D., \& Sanabria, D. (2016). Heart rate variability and cognitive processing: The autonomic response to task demands. Biological Psychology, 113, 83-90.

https://doi.org/10.1016/j.biopsycho.2015.11.013

MacDonald, Hultsch, D. F., \& Dixon, R. A. (2003). Performance variability is related to change in cognition: evidence from the Victoria Longitudinal Study. Psychology and Aging, 18(3), 510-523. https://doi.org/10.1037/0882-7974.18.3.510

Macdonald, S. W. S., Hultsch, D. F., \& Dixon, R. A. (2008). Predicting impending death: inconsistency in speed is a selective and early marker. Psychology and Aging, 23(3), 595-607. https://doi.org/10.1037/0882-7974.23.3.595

MacDonald, S. W. S., Li, S.-C., \& Bäckman, L. (2009). Neural underpinnings of withinperson variability in cognitive functioning. Psychology and Aging, 24(4), 792-808. https://doi.org/10.1037/a0017798

MacDonald, S. W. S., Nyberg, L., \& Bäckman, L. (2006). Intra-individual variability in behavior: links to brain structure, neurotransmission and neuronal activity. Trends in Neurosciences, 29(8), 474-480. https://doi.org/10.1016/j.tins.2006.06.011

Makin, S. M., Redman, J., Mosimann, U. P., Dudley, R., Clarke, M. P., Colbourn, C., \& Collerton, D. (2013). Complex visual hallucinations and attentional performance in eye disease and dementia: a test of the Perception and Attention Deficit model. International Journal of Geriatric Psychiatry, 28(12), 1232-1238. https://doi.org/10.1002/gps.3947

Malik, M., Cripps, T., Farrell, T., \& Camm, A. J. (1989). Prognostic value of heart rate variability after myocardial infarction. A comparison of different data-processing 
methods. Medical and Biological Engineering and Computing, 27(6), 603. https://doi.org/10.1007/BF02441642

Manni, R., Terzaghi, M., Ratti, P.-L., Repetto, A., Zangaglia, R., \& Pacchetti, C. (2011). Hallucinations and REM sleep behaviour disorder in Parkinson's disease: dream imagery intrusions and other hypotheses. Consciousness and Cognition, 20(4), 1021-1026. https://doi.org/10.1016/j.concog.2010.10.009

Maria, B., Sophia, S., Michalis, M., Charalampos, L., Andreas, P., John, M. E., \& Nikolaos, S. M. (2003). Sleep breathing disorders in patients with idiopathic Parkinson's disease. Respiratory Medicine, 97(10), 1151-1157.

Matsui, H., Udaka, F., Tamura, A., Oda, M., Kubori, T., Nishinaka, K., \& Kameyama, M. (2006). Impaired visual acuity as a risk factor for visual hallucinations in Parkinson's disease. Journal of Geriatric Psychiatry and Neurology, 19(1), 36-40. https://doi.org/10.1177/0891988705284739

Mazaheri, A., Segaert, K., Olichney, J., Yang, J.-C., Niu, Y.-Q., Shapiro, K., \& Bowman, H. (2018). EEG oscillations during word processing predict $\mathrm{MCl}$ conversion to Alzheimer's disease. Neurolmage: Clinical, 17, 188-197. https://doi.org/10.1016/j.nicl.2017.10.009

McIntosh, A.R., Kovacevic, N. \& Itier, R.J. (2008). Increased Brain Signal Variability Accompanies Lower Behavioral Variability in Development. PLOS Computational Biology, 4(7): e 1000106. https://doi:10.1371/journal.pcbi.10000106

Melgari, J.-M., Curcio, G., Mastrolilli, F., Salomone, G., Trotta, L., Tombini, M., ... Vernieri, F. (2014). Alpha and beta EEG power reflects L-dopa acute 
administration in parkinsonian patients. Frontiers in Aging Neuroscience, 6, 302. https://doi.org/10.3389/fnagi.2014.00302

Meppelink, A. M., de Jong, B. M., Renken, R., Leenders, K. L., Cornelissen, F. W., \& van Laar, T. (2009). Impaired visual processing preceding image recognition in Parkinson's disease patients with visual hallucinations. Brain: A Journal of Neurology, 132(Pt 11), 2980-2993. https://doi.org/10.1093/brain/awp223 Meppelink, A. M., Koerts, J., Borg, M., Leenders, K. L., \& van Laar, T. (2008). Visual object recognition and attention in Parkinson's disease patients with visual hallucinations. Movement Disorders: Official Journal of the Movement Disorder Society, 23(13), 1906-1912. https://doi.org/10.1002/mds.22270

Mihci, E., Kardelen, F., Dora, B., \& Balkan, S. (2006). Orthostatic heart rate variability analysis in idiopathic Parkinson's disease. Acta Neurologica Scandinavica, 113(5), 288-293. https://doi.org/10.1111/j.1600-0404.2006.00580.x

Miloserdov, K., Schmidt-Samoa, C., Williams, K., Weinrich, C., Bürk, K., Trenkwalder, C., ... Wilke, M. (2019). Misperceptions and Intra-Individual variability in Parkinson's disease: a behavioral and fMRI study. Manuscript submitted for publication.

Moeller, S., Yacoub, E., Olman, C. A., Auerbach, E., Strupp, J., Harel, N., \& Uğurbil, K. (2010). Multiband multislice GE-EPI at 7 tesla, with 16 -fold acceleration using partial parallel imaging with application to high spatial and temporal whole-brain fMRI. Magnetic Resonance in Medicine, 63(5), 1144-1153. https://doi.org/10.1002/mrm.22361

Moisello, C., Blanco, D., Lin, J., Panday, P., Kelly, S. P., Quartarone, A., ... Ghilardi, M. F. (2015). Practice changes beta power at rest and its modulation during 
movement in healthy subjects but not in patients with Parkinson's disease. Brain and Behavior, 5(10), e00374. https://doi.org/10.1002/brb3.374

Montoya, P., Schandry, R., \& Müller, A. (1993). Heartbeat evoked potentials (HEP): topography and influence of cardiac awareness and focus of attention. Electroencephalography and Clinical Neurophysiology, 88(3), 163-172.

Montse, A., Pere, V., Carme, J., Francesc, V., \& Eduardo, T. (2001). Visuospatial deficits in Parkinson's disease assessed by judgment of line orientation test: error analyses and practice effects. Journal of Clinical and Experimental Neuropsychology, 23(5), 592-598. https://doi.org/10.1076/jcen.23.5.592.1248 Morrison, A. P., Wells, A., \& Nothard, S. (2000). Cognitive factors in predisposition to auditory and visual hallucinations. The British Journal of Clinical Psychology, 39 ( Pt 1), 67-78.

Morrison, C., Rabipour, S., Taler, V., Sheppard, C., \& Knoefel, F. (2019). Visual EventRelated Potentials in Mild Cognitive Impairment and Alzheimer's Disease: A Literature Review. Current Alzheimer Research, 16(1), 67-89. https://doi.org/10.2174/1567205015666181022101036

Muller, A. J., Shine, J. M., Halliday, G. M., \& Lewis, S. J. G. (2014). Visual hallucinations in Parkinson's disease: theoretical models. Movement Disorders: Official Journal of the Movement Disorder Society, 29(13), 1591-1598. https://doi.org/10.1002/mds.26004

Musaeus, C. S., Engedal, K., Høgh, P., Jelic, V., Mørup, M., Naik, M., ... Andersen, B. B. (2018). EEG Theta Power Is an Early Marker of Cognitive Decline in Dementia due to Alzheimer's Disease. Journal of Alzheimer's Disease: JAD, 64(4), 13591371. https://doi.org/10.3233/JAD-180300 
Musso, M., Westervelt, H. J., Long, J. D., Morgan, E., Woods, S. P., Smith, M. M., ... PREDICT-HD Investigators of the Huntington Study Group. (2015). Intraindividual Variability in Prodromal Huntington Disease and Its Relationship to Genetic Burden. Journal of the International Neuropsychological Society: JINS, 21(1), 8-21. https://doi.org/10.1017/S1355617714001076

Nagatsu, T., Nakashima, A., Ichinose, H., \& Kobayashi, K. (2018). Human tyrosine hydroxylase in Parkinson's disease and in related disorders. Journal of Neural Transmission (Vienna, Austria: 1996). https://doi.org/10.1007/s00702-018-1903-3 Nagel, I. E., Preuschhof, C., Li, S.-C., Nyberg, L., Bäckman, L., Lindenberger, U., \& Heekeren, H. R. (2009). Performance level modulates adult age differences in brain activation during spatial working memory. Proceedings of the National Academy of Sciences of the United States of America, 106(52), 22552-22557. https://doi.org/10.1073/pnas.0908238106

Nègre-Pagès, L., Regragui, W., Bouhassira, D., Grandjean, H., Rascol, O., \& DoPaMiP Study Group. (2008). Chronic pain in Parkinson's disease: the cross-sectional French DoPaMiP survey. Movement Disorders: Official Journal of the Movement Disorder Society, 23(10), 1361-1369. https://doi.org/10.1002/mds.22142

Nishioka, K., Tanaka, R., Shimura, H., Hirano, K., Hatano, T., Miyakawa, K., ... Urabe, T. (2014). Quantitative evaluation of electroconvulsive therapy for Parkinson's disease with refractory psychiatric symptoms. Journal of Neural Transmission (Vienna, Austria: 1996), 121(11), 1405-1410. https://doi.org/10.1007/s00702-014$1212-4$

Obeso, J. A., Stamelou, M., Goetz, C. G., Poewe, W., Lang, A. E., Weintraub, D., ... Stoessl, A. J. (2017). Past, present, and future of Parkinson's disease: A special 
essay on the 200th Anniversary of the Shaking Palsy. Movement Disorders: Official Journal of the Movement Disorder Society, 32(9), 1264-1310. https://doi.org/10.1002/mds. 27115

Onofrj, M., Taylor, J. P., Monaco, D., Franciotti, R., Anzellotti, F., Bonanni, L., ... Thomas, A. (2013). Visual hallucinations in PD and Lewy body dementias: old and new hypotheses. Behavioural Neurology, 27(4), 479-493. https://doi.org/10.3233/BEN-129022

Oostenveld, R., Fries, P., Maris, E., \& Schoffelen, J.-M. (2011). FieldTrip: Open source software for advanced analysis of MEG, EEG, and invasive electrophysiological data. Computational Intelligence and Neuroscience, 2011, 156869. https://doi.org/10.1155/2011/156869

Pagonabarraga, J., Kulisevsky, J., Strafella, A. P., \& Krack, P. (2015). Apathy in Parkinson's disease: clinical features, neural substrates, diagnosis, and treatment. The Lancet. Neurology, 14(5), 518-531. https://doi.org/10.1016/S1474-4422(15)00019-8

Pagonabarraga, J., Martinez-Horta, S., Fernández de Bobadilla, R., Pérez, J., RibosaNogué, R., Marín, J., ... Kulisevsky, J. (2016). Minor hallucinations occur in drugnaive Parkinson's disease patients, even from the premotor phase. Movement Disorders: Official Journal of the Movement Disorder Society, 31(1), 45-52. https://doi.org/10.1002/mds.26432

Papapetropoulos, S., Katzen, H., Schrag, A., Singer, C., Scanlon, B. K., Nation, D., ... Levin, B. (2008). A questionnaire-based (UM-PDHQ) study of hallucinations in Parkinson's disease. BMC Neurology, 8, 21. https://doi.org/10.1186/1471-2377-821 
Papenberg, G., Bäckman, L., Chicherio, C., Nagel, I. E., Heekeren, H. R., Lindenberger, U., \& Li, S.-C. (2011). Higher intraindividual variability is associated with more forgetting and dedifferentiated memory functions in old age. Neuropsychologia, 49(7), 1879-1888. https://doi.org/10.1016/j.neuropsychologia.2011.03.013

Park, A., \& Stacy, M. (2009). Non-motor symptoms in Parkinson's disease. Journal of Neurology, 256 Supp/ 3, 293-298. https://doi.org/10.1007/s00415-009-5240-1

Parkinson Study Group. (1999). Low-dose clozapine for the treatment of drug-induced psychosis in Parkinson's disease. The New England Journal of Medicine, 340(10), 757-763. https://doi.org/10.1056/NEJM199903113401003

Peraza, L. R., Cromarty, R., Kobeleva, X., Firbank, M. J., Killen, A., Graziadio, S., ... Taylor, J.-P. (2018). Electroencephalographic derived network differences in Lewy body dementia compared to Alzheimer's disease patients. Scientific Reports, 8(1), 4637. https://doi.org/10.1038/s41598-018-22984-5

Peraza, L. R., Kaiser, M., Firbank, M., Graziadio, S., Bonanni, L., Onofrj, M., ... Taylor, J.-P. (2014). fMRI resting state networks and their association with cognitive fluctuations in dementia with Lewy bodies. Neurolmage. Clinical, 4, 558-565. https://doi.org/10.1016/j.nicl.2014.03.013

Pieri, V., Diederich, N. J., Raman, R., \& Goetz, C. G. (2000). Decreased color discrimination and contrast sensitivity in Parkinson's disease. Journal of the Neurological Sciences, 172(1), 7-11.

Pirogovsky-Turk, E., Moore, R. C., Filoteo, J. V., Litvan, I., Song, D. D., Lessig, S. L., \& Schiehser, D. M. (2017). Neuropsychiatric Predictors of Cognitive Decline in Parkinson Disease: A Longitudinal Study. The American Journal of Geriatric 
Psychiatry: Official Journal of the American Association for Geriatric Psychiatry, 25(3), 279-289. https://doi.org/10.1016/j.jagp.2016.10.004

Politis, M., Loane, C., Wu, K., O’Sullivan, S. S., Woodhead, Z., Kiferle, L., ... Piccini, P. (2013). Neural response to visual sexual cues in dopamine treatment-linked hypersexuality in Parkinson's disease. Brain: A Journal of Neurology, 136(Pt 2), 400-411. https://doi.org/10.1093/brain/aws326

Pollak, P., Tison, F., Rascol, O., Destée, A., Péré, J. J., Senard, J. M., ... Bourdeix, I. (2004). Clozapine in drug induced psychosis in Parkinson's disease: a randomised, placebo controlled study with open follow up. Journal of Neurology, Neurosurgery, and Psychiatry, 75(5), 689-695.

Pollock, B. G., Mulsant, B. H., Rosen, J., Mazumdar, S., Blakesley, R. E., Houck, P. R., \& Huber, K. A. (2007). A double-blind comparison of citalopram and risperidone for the treatment of behavioral and psychotic symptoms associated with dementia. The American Journal of Geriatric Psychiatry: Official Journal of the American Association for Geriatric Psychiatry, 15(11), 942-952. https://doi.org/10.1097/JGP.0b013e3180cc1ff5

Porges, S. W. (2007). A phylogenetic journey through the vague and ambiguous Xth cranial nerve: a commentary on contemporary heart rate variability research. Biological Psychology, 74(2), 301-307. https://doi.org/10.1016/j.biopsycho.2006.08.007

Power, J. D., Barnes, K. A., Snyder, A. Z., Schlaggar, B. L., \& Petersen, S. E. (2012). Spurious but systematic correlations in functional connectivity MRI networks arise from subject motion. Neurolmage, 59(3), 2142-2154. https://doi.org/10.1016/j.neuroimage.2011.10.018 
Pozzi, D., Petracchi, M., Sabe, L., Dancygier, G., García, H., \& Starkstein, S. (1994). Quantified electroencephalographic changes in Parkinson's disease with and without dementia. European Journal of Neurology, 1(2), 147-152. https://doi.org/10.1111/j.1468-1331.1994.tb00063.x

Prell, T. (2018). Structural and Functional Brain Patterns of Non-Motor Syndromes in Parkinson's Disease. Frontiers in Neurology, 9, 138. https://doi.org/10.3389/fneur.2018.00138

Ramirez-Ruiz, B., Junque, C., Marti, M.-J., Valldeoriola, F., \& Tolosa, E. (2007).

Cognitive changes in Parkinson's disease patients with visual hallucinations. Dementia and Geriatric Cognitive Disorders, 23(5), 281-288. https://doi.org/10.1159/000100850

Reichmann, H., Brandt, M. D., \& Klingelhoefer, L. (2016). The nonmotor features of Parkinson's disease: pathophysiology and management advances. Current Opinion in Neurology, 29(4), 467-473. https://doi.org/10.1097/WCO.0000000000000348

Reitan, R.M., \& Wolfson, D. (1993). The Halstead-Reitan Neuropsychological Test Battery: Theory and Clinical Interpretation 2nd edition. Neuropsychology Ress, Tuscon.

Rentrop, M., Rodewald, K., Roth, A., Simon, J., Walther, S., Fiedler, P., ... Kaiser, S. (2010). Intra-individual variability in high-functioning patients with schizophrenia. Psychiatry Research, 178(1), 27-32. https://doi.org/10.1016/j.psychres.2010.04.009

Romenets, S. R., Gagnon, J.-F., Latreille, V., Panniset, M., Chouinard, S., Montplaisir, J., \& Postuma, R. B. (2012). Rapid eye movement sleep behavior disorder and 
subtypes of Parkinson's disease. Movement Disorders: Official Journal of the Movement Disorder Society, 27(8), 996-1003. https://doi.org/10.1002/mds.25086

Saavedra, C., Olivares, E. I., \& Iglesias, J. (2012). Cognitive decline effects at an early stage: evidence from N170 and VPP. Neuroscience Letters, 518(2), 149-153. https://doi.org/10.1016/j.neulet.2012.04.075

Saliasi, E., Geerligs, L., Lorist, M. M., \& Maurits, N. M. (2013). The relationship between P3 amplitude and working memory performance differs in young and older adults. PloS One, 8(5), e63701. https://doi.org/10.1371/journal.pone.0063701

Salti, M., Bar-Haim, Y., \& Lamy, D. (2012). The P3 component of the ERP reflects conscious perception, not confidence. Consciousness and Cognition, 21(2), 961968. https://doi.org/10.1016/j.concog.2012.01.012

Sanchez-Ramos, J. R., Ortoll, R., \& Paulson, G. W. (1996). Visual hallucinations associated with Parkinson disease. Archives of Neurology, 53(12), 1265-1268.

Santangelo, G., Trojano, L., Vitale, C., lanniciello, M., Amboni, M., Grossi, D., \& Barone, P. (2007). A neuropsychological longitudinal study in Parkinson's patients with and without hallucinations. Movement Disorders: Official Journal of the Movement Disorder Society, 22(16), 2418-2425. https://doi.org/10.1002/mds.21746

Santangelo, G., Vitale, C., Picillo, M., Cuoco, S., Moccia, M., Pezzella, D., ... Pappatà, S. (2015). Apathy and striatal dopamine transporter levels in de-novo, untreated Parkinson's disease patients. Parkinsonism \& Related Disorders, 21(5), 489-493. https://doi.org/10.1016/j.parkreldis.2015.02.015

Schurger, A., Sarigiannidis, I., Naccache, L., Sitt, J. D., \& Dehaene, S. (2015). Cortical activity is more stable when sensory stimuli are consciously perceived. 
Proceedings of the National Academy of Sciences of the United States of America, 112(16), E2083-2092. https://doi.org/10.1073/pnas.1418730112

Schwartzman, D., Maravic, K., Kranczioch, C., \& Barnes, J. (2008). Altered early visual processing components in hallucination-prone individuals. NeuroReport, 19(9), 933. https://doi.org/10.1097/WNR.0b013e328301a640

Seeley, W. W., Menon, V., Schatzberg, A. F., Keller, J., Glover, G. H., Kenna, H., ... Greicius, M. D. (2007). Dissociable intrinsic connectivity networks for salience processing and executive control. The Journal of Neuroscience: The Official Journal of the Society for Neuroscience, 27(9), 2349-2356. https://doi.org/10.1523/JNEUROSCI.5587-06.2007

Sethi, K. D. (2002). Clinical aspects of Parkinson disease. Current Opinion in Neurology, 15(4), 457-460.

Setsompop, K., Gagoski, B. A., Polimeni, J. R., Witzel, T., Wedeen, V. J., \& Wald, L. L. (2012). Blipped-controlled aliasing in parallel imaging for simultaneous multislice echo planar imaging with reduced g-factor penalty. Magnetic Resonance in Medicine, 67(5), 1210-1224. https://doi.org/10.1002/mrm.23097

Shaffer, F., McCraty, R., \& Zerr, C. L. (2014). A healthy heart is not a metronome: an integrative review of the heart's anatomy and heart rate variability. Frontiers in Psychology, 5, 1040. https://doi.org/10.3389/fpsyg.2014.01040

Shannon, B. J., \& Buckner, R. L. (2004). Functional-anatomic correlates of memory retrieval that suggest nontraditional processing roles for multiple distinct regions within posterior parietal cortex. The Journal of Neuroscience: The Official Journal of the Society for Neuroscience, 24(45), 10084-10092. https://doi.org/10.1523/JNEUROSCI.2625-04.2004 
Shin, Y. S., Kim, S. N., Shin, N. Y., Jung, W. H., Hur, J.-W., Byun, M. S., .. Kwon, J. S. (2013). Increased intra-individual variability of cognitive processing in subjects at risk mental state and schizophrenia patients. PloS One, 8(11), e78354. https://doi.org/10.1371/journal.pone.0078354

Shine, J. M., Halliday, G. H., Carlos, M., Naismith, S. L., \& Lewis, S. J. G. (2012). Investigating visual misperceptions in Parkinson's disease: a novel behavioral paradigm. Movement Disorders: Official Journal of the Movement Disorder Society, 27(4), 500-505. https://doi.org/10.1002/mds.24900

Shine, J. M., Halliday, G. M., Gilat, M., Matar, E., Bolitho, S. J., Carlos, M., ... Lewis, S. J. G. (2014). The role of dysfunctional attentional control networks in visual misperceptions in Parkinson's disease. Human Brain Mapping, 35(5), 2206-2219. https://doi.org/10.1002/hbm.22321

Shine, J. M., Keogh, R., O’Callaghan, C., Muller, A. J., Lewis, S. J. G., \& Pearson, J. (2015). Imagine that: elevated sensory strength of mental imagery in individuals with Parkinson's disease and visual hallucinations. Proceedings. Biological Sciences / The Royal Society, 282(1798), 20142047. https://doi.org/10.1098/rspb.2014.2047

Shine, J. M., Muller, A. J., O'Callaghan, C., Hornberger, M., Halliday, G. M., \& Lewis, S. J. (2015). Abnormal connectivity between the default mode and the visual system underlies the manifestation of visual hallucinations in Parkinson's disease: a taskbased fMRI study. NPJ Parkinson's Disease, 1, 15003. https://doi.org/10.1038/npjparkd.2015.3 
Shine, J. M., O'Callaghan, C., Halliday, G. M., \& Lewis, S. J. G. (2014). Tricks of the mind: Visual hallucinations as disorders of attention. Progress in Neurobiology, 116, 58-65. https://doi.org/10.1016/j.pneurobio.2014.01.004

Simmonds, D. J., Fotedar, S. G., Suskauer, S. J., Pekar, J. J., Denckla, M. B., \& Mostofsky, S. H. (2007). Functional brain correlates of response time variability in children. Neuropsychologia, 45(9), 2147-2157. https://doi.org/10.1016/j.neuropsychologia.2007.01.013

Singh, A., \& Sørensen, T. L. (2011). Charles Bonnet syndrome improves when treatment is effective in age-related macular degeneration. The British Journal of Ophthalmology, 95(2), 291-292. https://doi.org/10.1136/bjo.2010.179465

Soikkeli, R., Partanen, J., Soininen, H., Pääkkönen, A., \& Riekkinen, P. (1991). Slowing of EEG in Parkinson's disease. Electroencephalography and Clinical Neurophysiology, 79(3), 159-165.

Speer, M. E., \& Soldan, A. (2015). Cognitive reserve modulates ERPs associated with verbal working memory in healthy younger and older adults. Neurobiology of Aging, 36(3), 1424-1434. https://doi.org/10.1016/j.neurobiolaging.2014.12.025

Sprengelmeyer, R., Young, A. W., Mahn, K., Schroeder, U., Woitalla, D., Büttner, T., ... Przuntek, H. (2003). Facial expression recognition in people with medicated and unmedicated Parkinson's disease. Neuropsychologia, 41(8), 1047-1057.

Stacy, M. (2002). Sleep disorders in Parkinson's disease: epidemiology and management. Drugs \& Aging, 19(10), 733-739. https://doi.org/10.2165/00002512-200219100-00002

Stamey, W., Davidson, A., \& Jankovic, J. (2008). Shoulder pain: a presenting symptom of Parkinson disease. Journal of Clinical Rheumatology: Practical Reports on 
Rheumatic \& Musculoskeletal Diseases, 14(4), 253-254.

https://doi.org/10.1097/RHU.0b013e3181826d43

Stein, T., Hebart, M. N., \& Sterzer, P. (2011). Breaking Continuous Flash Suppression:

A New Measure of Unconscious Processing during Interocular Suppression?

Frontiers in Human Neuroscience, 5, 167.

https://doi.org/10.3389/fnhum.2011.00167

Stothart, G., Tales, A., Hedge, C., \& Kazanina, N. (2014). Double peaked P1 visual evoked potentials in healthy ageing. Clinical Neurophysiology: Official Journal of the International Federation of Clinical Neurophysiology, 125(7), 1471-1478. https://doi.org/10.1016/j.clinph.2013.11.029

Stowe, R. L., Ives, N. J., Clarke, C., van Hilten, J., Ferreira, J., Hawker, R. J., ... Gray, R. (2008). Dopamine agonist therapy in early Parkinson's disease. The Cochrane Database of Systematic Reviews, (2), CD006564. https://doi.org/10.1002/14651858.CD006564.pub2

Stroop, J. R. (1935). Studies of interference in serial verbal reactions. Journal of Experimental Psychology, 18(6), 643-662. https://doi.org/10.1037/h0054651

Swann, N., Poizner, H., Houser, M., Gould, S., Greenhouse, I., Cai, W., ... Aron, A. R. (2011). Deep brain stimulation of the subthalamic nucleus alters the cortical profile of response inhibition in the beta frequency band: a scalp EEG study in Parkinson's disease. The Journal of Neuroscience: The Official Journal of the Society for Neuroscience, 31(15), 5721-5729. https://doi.org/10.1523/JNEUROSCI.6135-10.2011 
Tagliazucchi, E., \& Laufs, H. (2014). Decoding wakefulness levels from typical fMRI resting-state data reveals reliable drifts between wakefulness and sleep. Neuron, 82(3), 695-708. https://doi.org/10.1016/j.neuron.2014.03.020

Tahmasian, M., Eickhoff, S. B., Giehl, K., Schwartz, F., Herz, D. M., Drzezga, A., ... Eickhoff, C. R. (2017). Resting-state functional reorganization in Parkinson's disease: An activation likelihood estimation meta-analysis. Cortex; a Journal Devoted to the Study of the Nervous System and Behavior, 92, 119-138. https://doi.org/10.1016/j.cortex.2017.03.016

Tales, A., Leonards, U., Bompas, A., Snowden, R. J., Philips, M., Porter, G., .. Bayer, A. (2012). Intra-Individual Reaction Time Variability in Amnestic Mild Cognitive Impairment: A Precursor to Dementia? Journal of Alzheimer's Disease, 32(2), 457-466. https://doi.org/10.3233/JAD-2012-120505

Tarvainen, M. P., Niskanen, J.-P., Lipponen, J. A., Ranta-Aho, P. O., \& Karjalainen, P. A. (2014). Kubios HRV--heart rate variability analysis software. Computer Methods and Programs in Biomedicine, 113(1), 210-220. https://doi.org/10.1016/j.cmpb.2013.07.024

Tegegne, B. S., Man, T., van Roon, A. M., Riese, H., \& Snieder, H. (2018). Determinants of heart rate variability in the general population: The Lifelines Cohort Study. Heart Rhythm, 15(10), 1552-1558. https://doi.org/10.1016/j.hrthm.2018.05.006

Tessitore, A., Giordano, A., De Micco, R., Caiazzo, G., Russo, A., Cirillo, M., ... Tedeschi, G. (2016). Functional connectivity underpinnings of fatigue in "DrugNaïve" patients with Parkinson's disease. Movement Disorders: Official Journal of the Movement Disorder Society, 31(10), 1497-1505. https://doi.org/10.1002/mds.26650 
Tessitore, A., Santangelo, G., De Micco, R., Giordano, A., Raimo, S., Amboni, M., ... Vitale, C. (2017). Resting-state brain networks in patients with Parkinson's disease and impulse control disorders. Cortex; a Journal Devoted to the Study of the Nervous System and Behavior, 94, 63-72. https://doi.org/10.1016/j.cortex.2017.06.008

Thayer, J. F., Hansen, A. L., Saus-Rose, E., \& Johnsen, B. H. (2009). Heart rate variability, prefrontal neural function, and cognitive performance: the neurovisceral integration perspective on self-regulation, adaptation, and health. Annals of Behavioral Medicine: A Publication of the Society of Behavioral Medicine, 37(2), 141-153. https://doi.org/10.1007/s12160-009-9101-z

Tomlinson, C. L., Stowe, R., Patel, S., Rick, C., Gray, R., \& Clarke, C. E. (2010). Systematic review of levodopa dose equivalency reporting in Parkinson's disease. Movement Disorders: Official Journal of the Movement Disorder Society, 25(15), 2649-2653. https://doi.org/10.1002/mds.23429

Trojano, L., \& Papagno, C. (2018). Cognitive and behavioral disorders in Parkinson's disease: an update. II: behavioral disorders. Neurological Sciences: Official Journal of the Italian Neurological Society and of the Italian Society of Clinical Neurophysiology, 39(1), 53-61. https://doi.org/10.1007/s10072-017-3155-7

Troyer, A. K., Moscovitch, M., \& Winocur, G. (1997). Clustering and switching as two components of verbal fluency: evidence from younger and older healthy adults. Neuropsychology, 11(1), 138-146.

Tsuchiya, N., \& Koch, C. (2005). Continuous flash suppression reduces negative afterimages. Nature Neuroscience, 8(8), 1096-1101. https://doi.org/10.1038/nn1500 
Uc, E. Y., Rizzo, M., Anderson, S. W., Qian, S., Rodnitzky, R. L., \& Dawson, J. D. (2005). Visual dysfunction in Parkinson disease without dementia. Neurology, 65(12), 1907-1913. https://doi.org/10.1212/01.wnl.0000191565.11065.11

Valappil, R. A., Black, J. E., Broderick, M. J., Carrillo, O., Frenette, E., Sullivan, S. S., ... Langston, J. W. (2010). Exploring the electrocardiogram as a potential tool to screen for premotor Parkinson's disease. Movement Disorders: Official Journal of the Movement Disorder Society, 25(14), 2296-2303. https://doi.org/10.1002/mds.23348

van Belle, J., van Raalten, T., Bos, D. J., Zandbelt, B. B., Oranje, B., \& Durston, S. (2015). Capturing the dynamics of response variability in the brain in ADHD. Neurolmage. Clinical, 7, 132-141. https://doi.org/10.1016/j.nicl.2014.11.014 van den Heuvel, M. P., \& Hulshoff Pol, H. E. (2010). Exploring the brain network: a review on resting-state fMRI functional connectivity. European Neuropsychopharmacology: The Journal of the European College of Neuropsychopharmacology, 20(8), 519-534.

https://doi.org/10.1016/j.euroneuro.2010.03.008

Vanderwal, T., Kelly, C., Eilbott, J., Mayes, L. C., \& Castellanos, F. X. (2015). Inscapes:

A movie paradigm to improve compliance in functional magnetic resonance imaging. Neurolmage, 122, 222-232.

https://doi.org/10.1016/j.neuroimage.2015.07.069

Varanese, S., Perfetti, B., Ghilardi, M. F., \& Di Rocco, A. (2011). Apathy, but not depression, reflects inefficient cognitive strategies in Parkinson's disease. PloS One, 6(3), e17846. https://doi.org/10.1371/journal.pone.0017846 
Varanese, S., Perfetti, B., Monaco, D., Thomas, A., Bonanni, L., Tiraboschi, P., \& Onofrj, M. (2010). Fluctuating cognition and different cognitive and behavioural profiles in Parkinson's disease with dementia: comparison of dementia with Lewy bodies and Alzheimer's disease. Journal of Neurology, 257(6), 1004-1011. https://doi.org/10.1007/s00415-010-5453-3

Vertes, R. P. (2005). Hippocampal theta rhythm: a tag for short-term memory. Hippocampus, 15(7), 923-935. https://doi.org/10.1002/hipo.20118 von Holzen, J. J., Capaldo, G., Wilhelm, M., \& Stute, P. (2016). Impact of endo- and exogenous estrogens on heart rate variability in women: a review. Climacteric: The Journal of the International Menopause Society, 19(3), 222-228. https://doi.org/10.3109/13697137.2016.1145206

Wåhlstedt, C., Thorell, L. B., \& Bohlin, G. (2009). Heterogeneity in ADHD: neuropsychological pathways, comorbidity and symptom domains. Journal of Abnormal Child Psychology, 37(4), 551-564. https://doi.org/10.1007/s10802-008$9286-9$

Weintraub, D., Papay, K., Siderowf, A., \& Parkinson's Progression Markers Initiative. (2013). Screening for impulse control symptoms in patients with de novo Parkinson disease: a case-control study. Neurology, 80(2), 176-180. https://doi.org/10.1212/WNL.0b013e31827b915c

Weintraub, D., Simuni, T., Caspell-Garcia, C., Coffey, C., Lasch, S., Siderowf, A., ... Parkinson's Progression Markers Initiative. (2015). Cognitive performance and neuropsychiatric symptoms in early, untreated Parkinson's disease. Movement Disorders: Official Journal of the Movement Disorder Society, 30(7), 919-927. https://doi.org/10.1002/mds.26170 
West, R., Murphy, K. J., Armilio, M. L., Craik, F. I., \& Stuss, D. T. (2002). Lapses of intention and performance variability reveal age-related increases in fluctuations of executive control. Brain and Cognition, 49(3), 402-419.

Wilimzig, C., Ragert, P., \& Dinse, H. R. (2012). Cortical topography of intracortical inhibition influences the speed of decision making. Proceedings of the National Academy of Sciences of the United States of America, 109(8), 3107-3112. https://doi.org/10.1073/pnas.1114250109

Wilke, M. (2012). An iterative jackknife approach for assessing reliability and power of FMRI group analyses. PloS One, 7(4), e35578. https://doi.org/10.1371/journal.pone.0035578

Williams, \& Lees, A. J. (2005). Visual hallucinations in the diagnosis of idiopathic Parkinson's disease: a retrospective autopsy study. The Lancet. Neurology, 4(10), 605-610. https://doi.org/10.1016/S1474-4422(05)70146-0

Williams, Warren, J. D., \& Lees, A. J. (2008). Using the presence of visual hallucinations to differentiate Parkinson's disease from atypical parkinsonism. Journal of Neurology, Neurosurgery \&amp; Psychiatry, 79(6), 652. https://doi.org/10.1136/jnnp.2007.124677

Wykowska, A., \& Schubö, A. (2012). Action intentions modulate allocation of visual attention: electrophysiological evidence. Frontiers in Psychology, 3, 379. https://doi.org/10.3389/fpsyg.2012.00379

Yan, T., Bi, X., Zhang, M., Wang, W., Yao, Z., Yang, W., \& Wu, J. (2016). Age-related oscillatory theta modulation of multisensory integration in frontocentral regions. Neuroreport, 27(11), 796-801. https://doi.org/10.1097/WNR.0000000000000609 
Yao, N., Cheung, C., Pang, S., Shek-kwan Chang, R., Lau, K. K., Suckling, J., ... McAlonan, G. M. (2016). Multimodal MRI of the hippocampus in Parkinson's disease with visual hallucinations. Brain Structure \& Function, 221(1), 287-300. https://doi.org/10.1007/s00429-014-0907-5

Yao, N., Pang, S., Cheung, C., Chang, R. S.-K., Lau, K. K., Suckling, J., ... Chua, S.-E. (2015). Resting activity in visual and corticostriatal pathways in Parkinson's disease with hallucinations. Parkinsonism \& Related Disorders, 21(2), 131-137. https://doi.org/10.1016/j.parkreldis.2014.11.020

Yao, N., Shek-Kwan Chang, R., Cheung, C., Pang, S., Lau, K. K., Suckling, J., ... McAlonan, G. M. (2014). The default mode network is disrupted in Parkinson's disease with visual hallucinations. Human Brain Mapping, 35(11), 5658-5666. https://doi.org/10.1002/hbm.22577

Yong, S. W., Yoon, J. K., An, Y. S., \& Lee, P. H. (2007). A comparison of cerebral glucose metabolism in Parkinson's disease, Parkinson's disease dementia and dementia with Lewy bodies. European Journal of Neurology, 14(12), 1357-1362. https://doi.org/10.1111/j.1468-1331.2007.01977.x

Zhang, J.-J., Ding, J., Li, J.-Y., Wang, M., Yuan, Y.-S., Zhang, L., ... Zhang, K.-Z. (2017). Abnormal Resting-State Neural Activity and Connectivity of Fatigue in Parkinson's Disease. CNS Neuroscience \& Therapeutics, 23(3), 241-247. https://doi.org/10.1111/cns.12666 


\section{Aknowledgemets}

First, I would like to thank Prof. Dr. Melanie Wilke for giving me the opportunity to shape the wild and free growing flowers of scientific interest into a cultivated and visible garden of insights. I also would like to thank the other members of my thesis committee Prof. Dr. Annekathrin Schacht and Prof. Dr. Hans-Jörg Scherberger for discussion about the different pathways in the garden and for helping to choose the right tools to cultivate the ideas in my new garden. I also would like to thank Prof. Dr. Matthias Bähr and Dr. Christiane Weinrich for their help to find and choose the right seeds. And of course I can barely express how grateful I am to Carsten Schmidt - Samoa who helped in so many ways to use the tools in the right way to be able to cultivate and not to unintentially destroy my garden. I would like to thank Dr. Dechent for advising with the rigth (water)pipelines. I thank Dr. Iris Steinmann, Dr. Kathleen Williams, Diana Toscano, Dr. Yurrany Cabral-Calderin, Severin Heumüller and Eva Poland to help me water and care for my vulnebarable plants of science and for magically supply an umbrella on the most dark and rainy days. I also would like to thank Ilona Pfahlert for being the lab mom on the successful and less successful gardening days and Britta Perl for making unforgatable images of the seeds. Words can not express how greatful I am for my parents who unconditionally love and loved me and always believed that I can get whereever I want to be no matter which clamate I have to manage. I like to thank to my sisters which this life gave me on my way: Kalonike Klocke, Anina Fiedler and Melanie Winkle. Finally, I thank 'Garfield' to be my sunshine. 


\section{Contributions}

The first study about visual misperceptions and behavioral variability in Parkinson's disease described in Chapter 2, was designed by Melanie Wilke (MW), Kristina Miloserdov (KM) and Mathias Bähr (MB). Carsten Schmidt-Samoa (CS), MW and KM programmed scripts for creation and presentation of the stimuli. Christiane Anne Weinrich (CW), MB, Claudia Trenkwalder (CT) and Kathrin Bürk (KB) supported patient recruitment. CW contributed significant to clinical assessment of the patients. KM planned and performed data collection. CS, MW and KM prepared the scripts for behavioral analyses. Kathleen Williams (KW) preprocessed the resting state $\mathrm{fMRI}$ data and performed functional connectivity analyses. KM performed behavioral analses and second level analyses of the resting state fMRI data. MW and KM wrote the first version of the manuscript. MW, CS, KW, MB, CW, KB and CT reviewed the manuscript and provided corrections. KM and MW corrected the manuscript.

The second study about cognitive aging decribed in Chapter 3, was designed by MW, KM and Annekathrin Schacht (AS). CS, KM, Iris Steinmann (IS) and Diana Toscano (DT) modified scripts from the first study for creation and presentation of the sitmuli. DT planned and performed and KM supervised and contributed to data collection. CS, DT and KM prepared the scripts for behavioral analyses. IS and DT prepared the scripts for EEG analyses. DT performed behavioral and EEG data analyses. KM, CS and IS prepared scripts for ECG analyses. KM performed ECG data analyses. KM wrote the first and by now solitude version of the manuscript. 


